This document was prepared in conjunction with work accomplished under Contract No. DE-AC09-96SR18500 with the U. S. Department of Energy.

\title{
DISCLAIMER
}

This report was prepared as an account of work sponsored by an agency of the United States Government. Neither the United States Government nor any agency thereof, nor any of their employees, nor any of their contractors, subcontractors or their employees, makes any warranty, express or implied, or assumes any legal liability or responsibility for the accuracy, completeness, or any third party's use or the results of such use of any information, apparatus, product, or process disclosed, or represents that its use would not infringe privately owned rights. Reference herein to any specific commercial product, process, or service by trade name, trademark, manufacturer, or otherwise, does not necessarily constitute or imply its endorsement, recommendation, or favoring by the United States Government or any agency thereof or its contractors or subcontractors. The views and opinions of authors expressed herein do not necessarily state or reflect those of the United States Government or any agency thereof. 


\section{FRIT DEVELOPMENT EFFORTS FOR SLUDGE BATCH 4 (SB4): OPERATING WINDOW ASSESSMENTS OF SCENARIOS LEADING UP TO THE SELECTED PREPARATION PLAN FOR SB4}

D.K. Peeler

T.B. Edwards

January 2006

Process Science and Engineering Section Savannah River National Laboratory Aiken, SC 29808
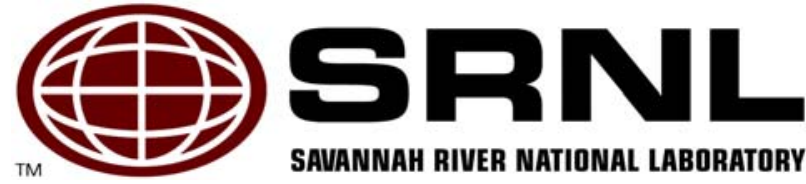
WSRC-RP-2006-00002

Revision 0

\section{DISCLAIMER}

This report was prepared by Washington Savannah River Company (WSRC) for the United States Department of Energy under Contract No. DE-AC09-96SR18500 and is an account of work performed under that contract. Neither the United States Department of Energy, nor WSRC, nor any of their employees makes any warranty, expressed or implied, or assumes any legal liability or responsibility for the accuracy, completeness, or usefulness, of any information, apparatus, or product or process disclosed herein or represents that its use will not infringe privately owned rights. Reference herein to any specific commercial product, process, or service by trademark, name, manufacturer or otherwise does not necessarily constitute or imply endorsement, recommendation, or favoring of same by WSRC or by the United States Government or any agency thereof. The views and opinions of the authors expressed herein do not necessarily state or reflect those of the United States Government or any agency thereof.

\section{Printed in the United States of America \\ Prepared For U.S. Department of Energy}

The Savannah River National Laboratory is operated for the U.S. Department of Energy by Washington Savannah River Company. 


\section{FRIT DEVELOPMENT EFFORTS FOR SLUDGE BATCH 4 (SB4): OPERATING WINDOW ASSESSMENTS OF SCENARIOS LEADING UP TO THE SELECTED PREPARATION PLAN FOR SB4}

D.K. Peeler

T.B. Edwards

January 2006

Process Science and Engineering Section Savannah River National Laboratory Aiken, SC 29808
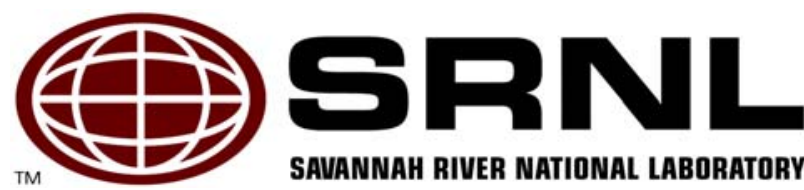


\section{REVIEWS AND APPROVALS}

\section{AUTHORS:}

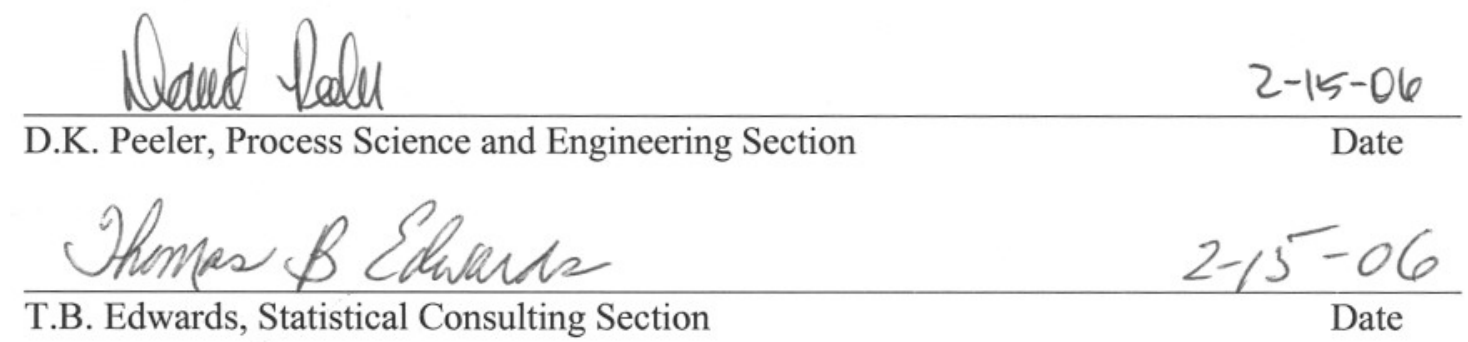

TECHNICAL REVIEWER:

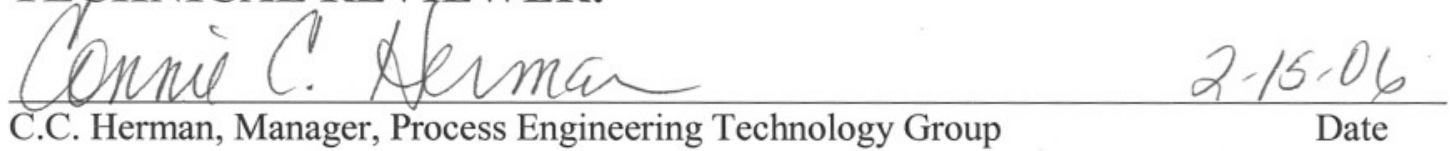

\section{APPROVERS:}
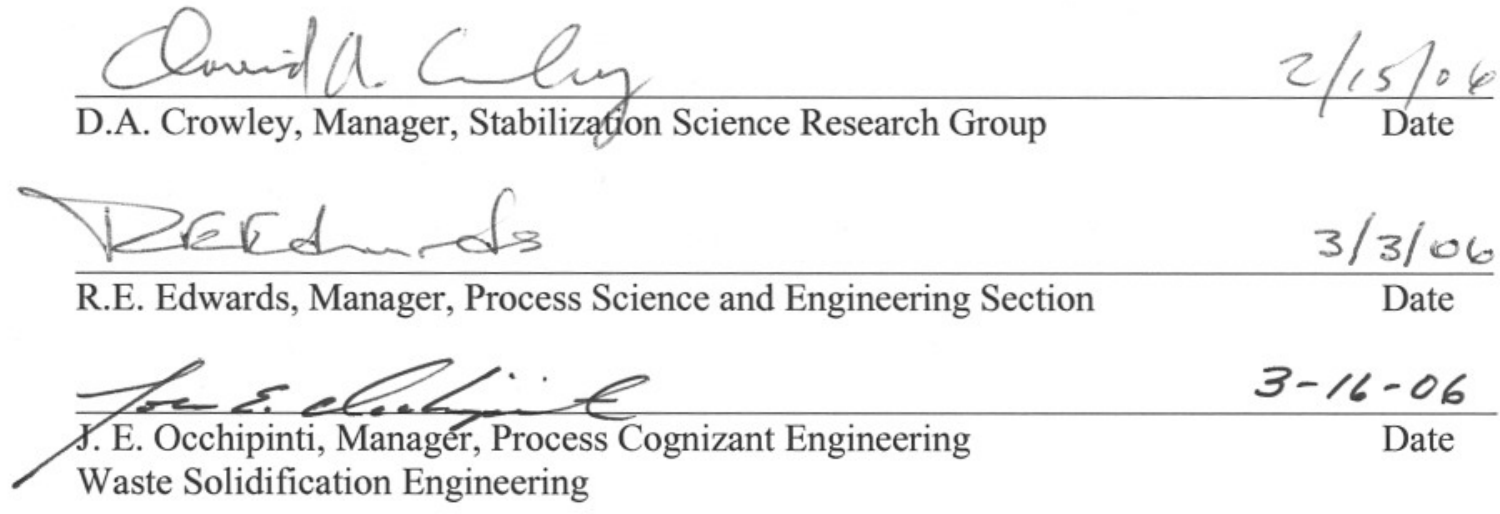
WSRC-RP-2006-00002

Revision 0

\section{EXECUTIVE SUMMARY}

The objective of this report is to document technical information that has been provided to Defense Waste Processing Facility (DWPF) and Closure Business Unit (CBU) personnel as part of the frit development support for Sludge Batch 4 (SB4). The information presented in this report includes projected operating windows (expressed in terms of waste loading) for various sludge blending and/or washing options coupled with candidate frits of interest. Although the Nominal Stage assessment serves as the primary tool for these evaluations, select systems were also evaluated using a Variation Stage assessment in which compositional variations were introduced. In addition, assessments of the impacts of nepheline formation potential and the $\mathrm{SO}_{4}{ }^{2}$ solubility limit on the projected operating windows are also provided.

Although this information was used as part of the technical basis leading to CBU's development of the preferred SB4 preparation plan, none of the options presented in this report was selected as the preferred plan. Therefore, the information is presented without significant interpretation of the resulting operating windows, but the projected windows are provided so additional insight can be explored if desired. Detailed assessments of the projected operating windows (using both Nominal and Variation Stage assessments) of the preferred sludge preparation plan with candidate frits are to be documented elsewhere.

The information provided in this report is focused solely on model-based projections of the operating windows for various SB4 blending strategies of interest. Although nepheline formation potential is monitored via model predictions as a part of this assessment, experimental work investigating the impact of nepheline on glass quality is also being addressed in a parallel study. The results of this paper study and the experimental assessments of melt rate, $\mathrm{SO}_{4}$ solubility, and/or nepheline formation potential are all critical components of the inputs into the frit selection process for SB4. 


\section{TABLE OF CONTENTS}

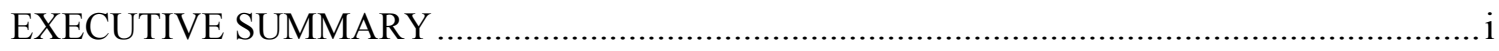

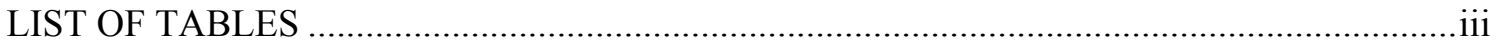

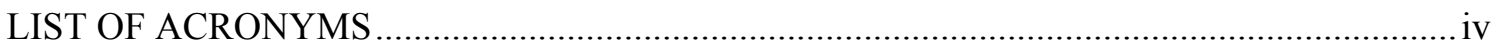

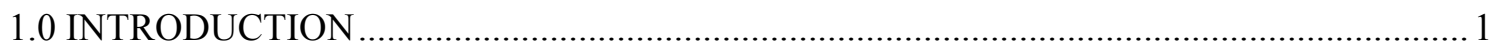

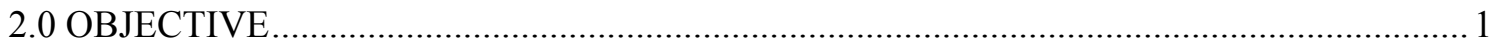

3.0 A PROGRESSION OF SB4 COMPOSITIONAL SCENARIOS …..................................... 2

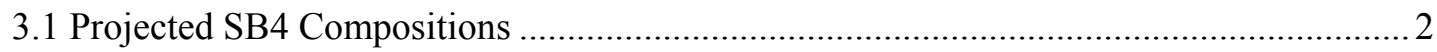

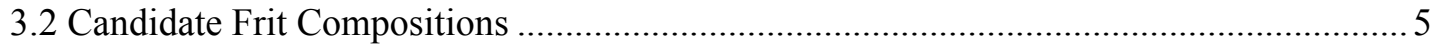

4.0 THE APPROACH AND CRITERIA FOR ACCEPTABILITY ….......................................... 6

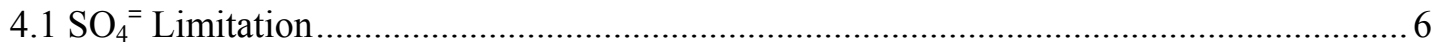

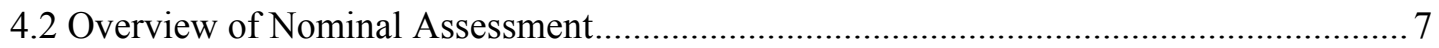

4.3 Overview of Variation Stage Assessment.................................................................... 7

5.0 PROJECTED OPERATING WINDOWS FOR THE SB4 OPTIONS ................................ 12

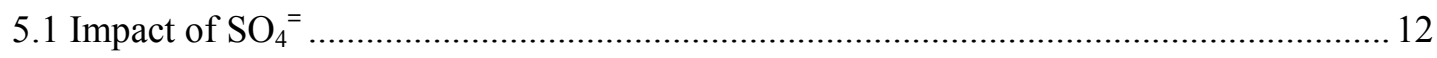

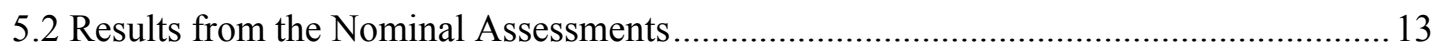

5.3 Results from the Variation Stage Assessments ............................................................. 19

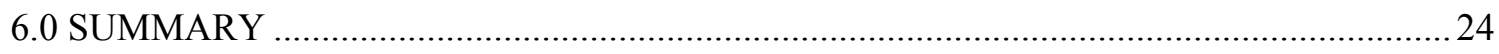

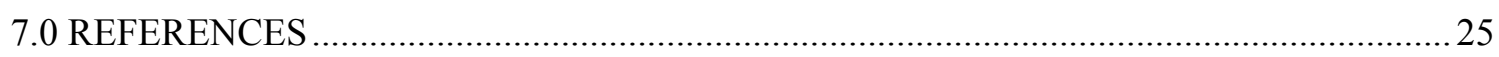

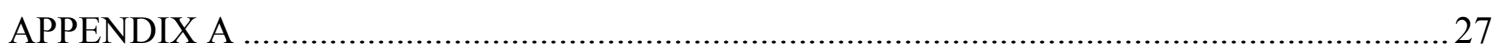




\section{LIST OF TABLES}

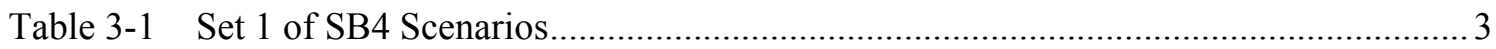

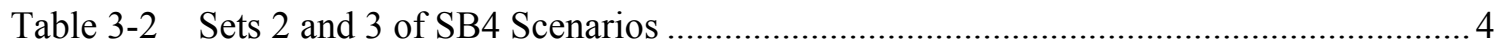

Table 3-3 Composition of Candidate Frits (oxide wt \%) ….................................................... 5

Table 4-1 Developing Bounding Regions for Case 15 and Case $7 \mathrm{~b}$ (as mass fractions) ............ 9

Table 4-2 Components (as weight percentages) of Others for Case 15 and Case 7b.............. 10

Table 4-3 Glass Systems Evaluated During Variation Stage Assessments.............................. 11

Table 5-1 $\mathrm{SO}_{4}{ }^{=}$Concentrations in the Glass for the Sludge Cases at Select WLs .................... 13

Table 5-2 Operating Windows from Nominal Assessments Expressed as WL Intervals

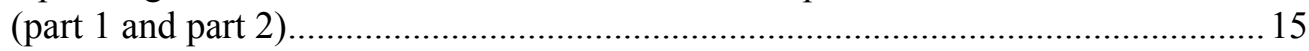

Table 5-3 Variation Stage MAR Results (as \% of EVs) for Case 15 Batch 1 and Blend 1 ..... 20

Table 5-4 Variation Stage MAR Results (as \% of EVs) for Case 15 Batch 2 and Blend 2 ...... 21

Table 5-5 Variation Stage MAR Results (as \% of EVs) for Case 7b Batch 1 and Blend 1 ...... 22

Table 5-6 Variation Stage MAR Results (as \% of EVs) for Case 7b Batch 2 and Blend 2 ...... 23

Table 5-7 Intervals with 100\% EVs Acceptable and Limiting Constraint(s)...........................2 24 


\section{LIST OF ACRONYMS}

$\begin{array}{ll}\text { ARP } & \text { Actinide Removal Process } \\ \text { CBU } & \text { Closure Business Unit } \\ \text { CPC } & \text { Chemical Process Cell } \\ \text { DWPF } & \text { Defense Waste Processing Facility } \\ \text { EV } & \text { Extreme Vertices } \\ \Delta G_{P} & \text { preliminary glass dissolution estimator } \\ \text { HLW } & \text { High Level Waste } \\ \text { MAR } & \text { Measurement Acceptability Region } \\ \text { MCU } & \text { Modular Caustic Side Solvent Extraction Unit } \\ \text { PCCS } & \text { Product Composition Control System } \\ \text { PCT } & \text { Product Consistency Test } \\ \text { SB } & \text { sludge batch } \\ \text { SRNL } & \text { Savannah River National Laboratory } \\ T_{L} & \text { liquidus temperature } \\ \eta & \text { viscosity } \\ \text { WL } & \text { waste loading }\end{array}$




\subsection{INTRODUCTION}

A preferred sludge preparation plan for the next sludge batch (Sludge Batch 4, SB4) of high-level waste (HLW) to be processed at the Savannah River Site's Defense Waste Processing Facility (DWPF) has been identified by the Closure Business Unit (CBU). ${ }^{1}$ A progression of various blending and washing strategies was evaluated by the CBU as it made its decision on the SB4 preparation plan (Shah 2006). The Savannah River National Laboratory (SRNL) provided technical assessment support to the CBU during this process. This support included assessments of candidate SB4 projected compositions that were conducted by SRNL's frit development team. SRNL was asked to provide support for SB4 via a technical task request (Washburn 2004), and SRNL responded to the request by issuing a task technical and quality assurance plan (Peeler 2004) under whose auspices this report was prepared. The purpose of this report is to document the most recent assessments that were completed by the frit development team of SB4 options leading up to the preferred sludge preparation plan. The assessments were strictly paper studies driven by predictions from glass property/glass composition models most of which are currently used by DWPF to control its process and the quality (i.e., the durability) of its final product - no experimental work was conducted to support the assessments documented in this report.

Objectives of the SB4 frit development task are specified in Section 2.0. The set of projected SB4 nominal compositions for which assessments were conducted is provided in Section 3.0. Also discussed in Section 3.0 are the candidate frits and the strategy behind their use. Section 4.0 provides a brief overview of how sludge/frit glass systems are assessed. There are two stages to the assessment: a Nominal Stage (a screening tool applied to a large set of candidate frits) and a Variation Stage (a more thorough assessment applied to select sludge/frit systems). Both assessments use the same criteria to make acceptability decisions that lead to a projected operating window (an interval of waste loading over which all the criteria are simultaneously satisfied) that may be achieved for a specific sludge/frit glass system. Section 5.0 summarizes the results of the assessments conducted for the various SB4 options. Issues associated with a $\mathrm{SO}_{4}{ }^{2}$ limit are also discussed in Section 5.0 with respect to how the limit may influence acceptable waste loading (WL) intervals. Section 6.0 provides a summary of this work that supported the CBU's decisions that led to the preferred SB4 preparation plan.

\subsection{OBJECTIVE}

SRNL has developed, and continues to enhance, its integrated capability to evaluate the impact of proposed sludge flowsheets on DWPF's operation for the CBU. The components of this support include frit development, Chemical Process Cell (CPC) flowsheet development, and melt rate assessment.

The objective of frit development is to identify a viable frit or frits for each sludge preparation option being contemplated. A frit is considered viable if its composition allows for economic fabrication and if, when it is combined with the sludge option under consideration, DWPF's property/composition models indicate that the combination has the potential for an operating window (a waste loading interval over which the sludge/frit glass system satisfies processability and durability constraints) that would allow DWPF to meet its goals for waste loading and canister production rate.

\footnotetext{
${ }^{1}$ Due to tank settling and washing issues, the strategy outlined by the CBU has evolved into the definition of a sludge preparation plan for the tanks proposed for SB4 which now involves preparation as SB4 and Sludge Batch 5 (SB5). Typically, four options were provided for each candidate preparation plan, which included Batch 1, Blend 1, Batch 2, and Blend 2. "Batches" refer to projected sludge compositions (prior to blending) that would be qualified. "Blends" refer to projected sludge compositions (after blending with the heel of the previous sludge batch) that would be processed in DWPF. For simplicity purposes in this report, the authors will refer to SB4 only in the discussions that follow given none of the cases defines the preferred preparation plan.
} 
The objective of this report is to document the results of SRNL's efforts to identify candidate frit compositions and corresponding predicted operating windows for various SB4 sludge preparation plan options that CBU entertained (Shah 2006) on its way to finalizing the SB4 preparation plan. The information provided in this report is a summary of and is limited to the model-based projections of operating windows that were provided to $\mathrm{CBU}$ for the candidate sludge preparation plans. Although this information was used as part of the technical basis from which the preferred SB4 preparation plan was selected, none of the options presented in this report is the preferred plan. Therefore the information is presented without significant interpretation but the details are provided so additional insight can be made if desired. Details regarding the evaluation of the preferred plan are provided by Peeler and Edwards (2006).

\subsection{A PROGRESSION OF SB4 COMPOSITIONAL SCENARIOS}

The compositional scenarios under consideration for SB4 progressed to the point that the some of the preparation plans developed by CBU involved up to two transfers to (or blends in) Tank 40 from Tank 51 and the qualification of two different batches of material in Tank 51 (Shah 2006). In the text that follows, the term "batch" refers to a projected nominal sludge composition that would be qualified (prior to blending with the heel of the previous sludge batch). The term "blend" refers to a projected nominal sludge composition of a blended sludge that would be processed in DWPF. The dual batches and/or blends are indicated by batch or blend number within a particular scenario in the discussion that follows. Frit assessments were conducted for three sets of SB4 scenarios. In this section, the nominal compositions defining each scenario are presented. The set of candidate frits that was used in the assessment of each of the sludge compositions is also presented in this section.

\subsection{Projected SB4 Compositions}

Shah (2006) provided the projected elemental compositions for the various sludge preparation options. To support the model based assessments, elemental concentrations were converted to oxide concentrations by multiplying the values for each element by the gravimetric factor for the corresponding oxide. $\mathrm{SO}_{4}{ }^{=}$concentrations were not provided by Shah (2006). However, CBU personnel did provide information as part of the washing scenarios and preparations plans that was used to derive estimates for the $\mathrm{SO}_{4}{ }$ concentrations. These concentrations were added to the oxide list and the resulting oxide concentrations were then normalized to 100\%. Tables 3-1 (Set 1) and 3-2 (Sets 2 and 3, which are revisions of the Set 1 projections based upon different mass limits for hydrogen generation and washing endpoints, respectively) provide the oxide compositions (in weight percent, wt $\%$ ) of the SB4 scenarios being considered in this report. A Case 15C was also included as part of Set 3 and represents an iteration of Case 15B and Case 15 with mass adjustments for hydrogen retention and less washing, respectively. However, this case was selected as the preferred sludge preparation plan for SB4, and its evaluation is the subject of a separate report (Peeler and Edwards 2006). It should be noted that the projected sludge compositions presented in Tables 3-1 and 3-2 are sludge-only flowsheets and do not account for any potential secondary streams from the Actinide Removal Process (ARP) or the Modular Caustic Side Solvent Extraction Unit (MCU). The assessment of the preferred SB4 preparation plan, which is addressed in a separate document (Peeler and Edwards 2006), will include evaluations of the impact of auxiliary ARP streams. Based upon the results presented by Peeler et al. (2005a), the addition of a MCU stream is not expected to have an impact on the projected operating windows for SB4. As seen in Table 3-1 and 3-2, the projected sludge compositions are relatively high in $\mathrm{Al}_{2} \mathrm{O}_{3}, \mathrm{Na}_{2} \mathrm{O}$, and $\mathrm{SO}_{4}{ }^{\circ}$ and relatively low in $\mathrm{Fe}_{2} \mathrm{O}_{3}$ (as compared to previous sludges processed in DWPF). In addition, the $\mathrm{SiO}_{2}$ content of the projected sludges typically ranges from 3 to $6.5 \mathrm{wt} \%$, which is at higher concentrations than previous sludge batches (which were normally in the $1-2 \mathrm{wt} \%$ range). 
Table 3-1. Set 1 of SB4 Scenarios

\begin{tabular}{|c|c|c|c|c|c|c|c|c|c|c|c|c|c|c|c|}
\hline \multirow[b]{2}{*}{ Oxide } & $\begin{array}{r}\text { Case } 8 \\
\text { and } 8 \mathrm{~A} \\
\end{array}$ & Case 8 & Case $8 \mathrm{a}$ & Case 7 & Case 7 & Case 7 & Case 7 & $\begin{array}{l}\text { Case } \\
15 \mathrm{~B} \\
\end{array}$ & $\begin{array}{l}\text { Case } \\
15 \mathrm{~B} \\
\end{array}$ & $\begin{array}{l}\text { Case } \\
15 \mathrm{~B} \\
\end{array}$ & $\begin{array}{l}\text { Case } \\
15 \mathrm{~B} \\
\end{array}$ & Case 16 & Case 16 & Case 16 & Case 16 \\
\hline & Batch 1 & Blend 1 & Blend 1 & Batch 1 & Blend 1 & Batch 2 & Blend 2 & Batch 1 & Blend 1 & Batch 2 & Blend 2 & Batch 1 & Blend 1 & Batch 2 & Blend 2 \\
\hline $\mathrm{Al}_{2} \mathrm{O}_{3}$ & 28.765 & 23.017 & 25.706 & 31.877 & 18.764 & 33.940 & 30.842 & 41.321 & 19.092 & 32.634 & 29.940 & 6.364 & 14.104 & 37.481 & 31.658 \\
\hline $\mathrm{BaO}$ & 0.151 & 0.148 & 0.149 & 0.132 & 0.143 & 0.141 & 0.140 & 0.091 & 0.138 & 0.145 & 0.143 & 0.219 & 0.172 & 0.113 & 0.127 \\
\hline $\mathrm{CaO}$ & 1.679 & 2.161 & 1.923 & 1.622 & 2.593 & 1.726 & 1.887 & 1.550 & 2.665 & 1.726 & 1.896 & 2.184 & 2.903 & 1.517 & 1.847 \\
\hline $\mathrm{Ce}_{2} \mathrm{O}_{3}$ & 0.065 & 0.135 & 0.101 & 0.046 & 0.195 & 0.049 & 0.077 & 0.000 & 0.200 & 0.054 & 0.082 & 0.181 & 0.240 & 0.018 & 0.071 \\
\hline $\mathrm{Cr}_{2} \mathrm{O}_{3}$ & 0.217 & 0.222 & 0.219 & 0.204 & 0.225 & 0.217 & 0.218 & 0.179 & 0.223 & 0.219 & 0.219 & 0.266 & 0.254 & 0.191 & 0.206 \\
\hline $\mathrm{CuO}$ & 0.035 & 0.059 & 0.047 & 0.025 & 0.079 & 0.026 & 0.037 & 0.000 & 0.080 & 0.029 & 0.039 & 0.096 & 0.101 & 0.010 & 0.032 \\
\hline $\mathrm{Fe}_{2} \mathrm{O}_{3}$ & 20.259 & 25.104 & 22.702 & 19.006 & 29.317 & 20.236 & 21.908 & 16.687 & 29.840 & 20.407 & 22.102 & 27.013 & 33.187 & 17.409 & 21.163 \\
\hline $\mathrm{K}_{2} \mathrm{O}$ & 0.415 & 0.327 & 0.368 & 0.455 & 0.261 & 0.484 & 0.439 & 0.578 & 0.263 & 0.467 & 0.427 & 0.167 & 0.211 & 0.520 & 0.443 \\
\hline $\mathrm{La}_{2} \mathrm{O}_{3}$ & 0.095 & 0.102 & 0.098 & 0.095 & 0.109 & 0.102 & 0.103 & 0.101 & 0.111 & 0.100 & 0.102 & 0.092 & 0.115 & 0.097 & 0.101 \\
\hline $\mathrm{MgO}$ & 1.473 & 2.305 & 1.899 & 1.250 & 3.013 & 1.331 & 1.651 & 0.739 & 3.085 & 1.385 & 1.700 & 3.484 & 3.737 & 0.797 & 1.507 \\
\hline $\mathrm{MnO}$ & 5.152 & 5.712 & 5.426 & 4.652 & 6.142 & 4.953 & 5.162 & 3.589 & 6.105 & 5.054 & 5.231 & 8.285 & 7.384 & 3.910 & 4.737 \\
\hline $\mathrm{Na}_{2} \mathrm{O}$ & 23.608 & 23.265 & 23.665 & 24.609 & 22.621 & 19.992 & 20.859 & 22.621 & 22.146 & 20.463 & 21.116 & 31.079 & 19.255 & 22.907 & 22.340 \\
\hline $\mathrm{NiO}$ & 3.925 & 3.008 & 3.439 & 3.145 & 2.044 & 3.349 & 3.081 & 1.298 & 1.667 & 3.552 & 3.181 & 5.075 & 2.601 & 2.668 & 2.641 \\
\hline $\mathrm{PbO}$ & 0.036 & 0.080 & 0.058 & 0.025 & 0.117 & 0.027 & 0.044 & 0.000 & 0.121 & 0.030 & 0.047 & 0.110 & 0.145 & 0.008 & 0.041 \\
\hline $\mathrm{SO}_{4}{ }^{=}$ & 1.121 & 1.138 & 1.124 & 0.965 & 1.124 & 0.766 & 0.833 & 1.649 & 1.246 & 0.867 & 0.934 & 0.302 & 0.725 & 1.174 & 1.060 \\
\hline $\mathrm{SiO}_{2}$ & 4.250 & 3.738 & 3.972 & 4.656 & 3.400 & 4.958 & 4.633 & 5.913 & 3.485 & 4.781 & 4.517 & 1.779 & 2.962 & 5.305 & 4.715 \\
\hline $\mathrm{ThO}_{2}$ & 0.075 & 0.058 & 0.066 & 0.087 & 0.045 & 0.093 & 0.083 & 0.121 & 0.047 & 0.088 & 0.080 & 0.000 & 0.028 & 0.106 & 0.087 \\
\hline $\mathrm{TiO}_{2}$ & 0.244 & 0.155 & 0.197 & 0.180 & 0.062 & 0.191 & 0.165 & 0.023 & 0.028 & 0.209 & 0.174 & 0.490 & 0.134 & 0.111 & 0.116 \\
\hline $\mathrm{U}_{3} \mathrm{O}_{8}$ & 8.140 & 8.919 & 8.518 & 6.709 & 9.360 & 7.143 & 7.541 & 3.359 & 9.073 & 7.506 & 7.770 & 12.324 & 11.277 & 5.446 & 6.837 \\
\hline $\mathrm{ZnO}$ & 0.058 & 0.097 & 0.078 & 0.041 & 0.129 & 0.044 & 0.060 & 0.000 & 0.131 & 0.048 & 0.064 & 0.163 & 0.166 & 0.015 & 0.052 \\
\hline $\mathrm{ZrO}_{2}$ & 0.237 & 0.249 & 0.243 & 0.219 & 0.257 & 0.233 & 0.237 & 0.182 & 0.255 & 0.236 & 0.239 & 0.327 & 0.299 & 0.197 & 0.221 \\
\hline & 100.000 & 100.000 & 100.000 & 100.000 & 100.000 & 100.000 & 100.000 & 100.000 & 100.000 & 100.000 & 100.000 & 100.000 & 100.000 & 100.000 & 100.000 \\
\hline
\end{tabular}


Table 3-2. Sets 2 and 3 of SB4 Scenarios

\begin{tabular}{|c|c|c|c|c|c|c|c|c|}
\hline \multirow[b]{3}{*}{ Oxide } & Set 2 & Set 2 & Set 2 & Set 2 & Set 3 & Set 3 & Set 3 & Set 3 \\
\hline & Case 15 & Case 15 & Case 15 & Case 15 & Case $7 b$ & Case $7 b$ & Case $7 b$ & Case $7 b$ \\
\hline & Batch 1 & Blend 1 & Batch 2 & Blend 2 & Batch 1 & Blend 1 & Batch 2 & Blend 2 \\
\hline $\mathrm{Al}_{2} \mathrm{O}_{3}$ & 44.265 & 25.362 & 28.491 & 27.705 & 30.874 & 20.810 & 32.933 & 30.114 \\
\hline $\mathrm{BaO}$ & 0.097 & 0.129 & 0.149 & 0.144 & 0.128 & 0.140 & 0.136 & 0.136 \\
\hline $\mathrm{CaO}$ & 1.660 & 2.441 & 1.663 & 1.814 & 1.571 & 2.396 & 1.675 & 1.825 \\
\hline $\mathrm{Ce}_{2} \mathrm{O}_{3}$ & 0.000 & 0.153 & 0.064 & 0.082 & 0.045 & 0.167 & 0.048 & 0.074 \\
\hline $\mathrm{Cr}_{2} \mathrm{O}_{3}$ & 0.192 & 0.217 & 0.215 & 0.215 & 0.197 & 0.219 & 0.211 & 0.211 \\
\hline $\mathrm{CuO}$ & 0.000 & 0.061 & 0.035 & 0.040 & 0.024 & 0.069 & 0.026 & 0.035 \\
\hline $\mathrm{Fe}_{2} \mathrm{O}_{3}$ & 17.876 & 27.162 & 20.066 & 21.421 & 18.408 & 27.203 & 19.635 & 21.198 \\
\hline $\mathrm{K}_{2} \mathrm{O}$ & 0.619 & 0.352 & 0.411 & 0.397 & 0.441 & 0.291 & 0.470 & 0.428 \\
\hline $\mathrm{La}_{2} \mathrm{O}_{3}$ & 0.108 & 0.111 & 0.094 & 0.097 & 0.092 & 0.105 & 0.099 & 0.100 \\
\hline $\mathrm{MgO}$ & 0.791 & 2.551 & 1.458 & 1.675 & 1.211 & 2.675 & 1.291 & 1.588 \\
\hline $\mathrm{MnO}$ & 3.845 & 5.603 & 5.103 & 5.180 & 4.506 & 5.814 & 4.806 & 5.003 \\
\hline $\mathrm{Na}_{2} \mathrm{O}$ & 17.610 & 20.489 & 24.775 & 24.274 & 26.766 & 23.771 & 22.175 & 22.912 \\
\hline $\mathrm{NiO}$ & 1.390 & 1.612 & 3.888 & 3.401 & 3.046 & 2.211 & 3.250 & 3.006 \\
\hline $\mathrm{PbO}$ & 0.000 & 0.093 & 0.035 & 0.047 & 0.025 & 0.100 & 0.026 & 0.042 \\
\hline $\mathrm{SO}_{4}{ }^{=}$ & 1.264 & 1.210 & 0.671 & 0.778 & 1.149 & 1.161 & 0.933 & 0.975 \\
\hline $\mathrm{SiO}_{2}$ & 6.335 & 4.205 & 4.209 & 4.187 & 4.510 & 3.578 & 4.810 & 4.517 \\
\hline $\mathrm{ThO}_{2}$ & 0.130 & 0.067 & 0.075 & 0.073 & 0.084 & 0.052 & 0.090 & 0.081 \\
\hline $\mathrm{TiO}_{2}$ & 0.025 & 0.027 & 0.242 & 0.197 & 0.174 & 0.081 & 0.186 & 0.162 \\
\hline $\mathrm{U}_{3} \mathrm{O}_{8}$ & 3.599 & 7.812 & 8.063 & 7.971 & 6.498 & 8.796 & 6.931 & 7.306 \\
\hline $\mathrm{ZnO}$ & 0.000 & 0.100 & 0.057 & 0.066 & 0.040 & 0.113 & 0.042 & 0.058 \\
\hline $\mathrm{ZrO}_{2}$ & 0.195 & 0.242 & 0.235 & 0.235 & 0.212 & 0.248 & 0.226 & 0.230 \\
\hline & 100.000 & 100.000 & 100.000 & 100.000 & 100.000 & 100.000 & 100.000 & 100.000 \\
\hline
\end{tabular}




\subsection{Candidate Frit Compositions}

Table 3-3 provides the list of candidate frits and nominal compositions (weight $\%$ basis) considered in this assessment. A closer review of frits X1-1 through 422 (shaded in Table 3-3) indicates fixed concentrations of $\mathrm{B}_{2} \mathrm{O}_{3}$ and $\mathrm{Li}_{2} \mathrm{O}$ at $8 \mathrm{wt} \%$ with only the $\mathrm{Na}_{2} \mathrm{O}$ and $\mathrm{SiO}_{2}$ concentrations varying. In general, these frit compositions increase in $\mathrm{Na}_{2} \mathrm{O}$ by $1 \%$ and decrease respectively in $\mathrm{SiO}_{2}$ proceeding from Frit X1-1 (the most refractory frit being considered) to Frit 422. This system has been referred to as a "sliding $\mathrm{Na}_{2} \mathrm{O}$ scale" concept which was developed to accommodate potential $\mathrm{Na}_{2} \mathrm{O}$ concentration differences in the sludge as a result of varying blending and/or washing strategies being considered (see Peeler and Edwards 2002 and 2005 for more details).

Frits 202 and 200 are historical frits that were developed to support both the coupled and sludgeonly flowsheets. They are being carried forward in this assessment to provide insight into their potential use with the SB4 options. The "P-series" of frits have been developed to minimize the potential for nepheline formation given its impact on durability (Peeler et al. (2005b and 2006)). In general, these frits have lower $\mathrm{Na}_{2} \mathrm{O}$ than the "sliding scale series" with the difference being accounted for by an increase in $\mathrm{Li}_{2} \mathrm{O}$ and/or $\mathrm{Fe}_{2} \mathrm{O}_{3}$. $\mathrm{Fe}_{2} \mathrm{O}_{3}$ was added as a "filler" given its inert effects on nepheline formation and its potentially advantageous effects on viscosity and/or melt rate.

Table 3-3. Composition of Candidate Frits (oxide wt\%)

\begin{tabular}{||c|c|c|c|c|c|c|}
\hline Frit & $\mathbf{B}_{2} \mathbf{O}_{3}$ & $\mathbf{F e}_{2} \mathbf{O}_{3}$ & $\mathbf{L i}_{2} \mathbf{O}$ & $\mathbf{M g O}$ & $\mathbf{N a}_{2} \mathbf{O}$ & $\mathbf{S i O}_{2}$ \\
\hline $\mathrm{X} 1-1$ & 8 & 0 & 8 & 0 & 15 & 69 \\
\hline $\mathrm{Y} 1-1$ & 8 & 0 & 8 & 0 & 14 & 70 \\
\hline 431 & 8 & 0 & 8 & 0 & 13 & 71 \\
\hline 320 & 8 & 0 & 8 & 0 & 12 & 72 \\
\hline 417 & 8 & 0 & 8 & 0 & 11 & 73 \\
\hline 425 & 8 & 0 & 8 & 0 & 10 & 74 \\
\hline 426 & 8 & 0 & 8 & 0 & 9 & 75 \\
\hline 418 & 8 & 0 & 8 & 0 & 8 & 76 \\
\hline 460 & 8 & 0 & 8 & 0 & 7 & 77 \\
\hline 432 & 8 & 0 & 8 & 0 & 6 & 78 \\
\hline 473 & 8 & 0 & 8 & 0 & 5 & 79 \\
\hline $\mathrm{d} 1-1$ & 8 & 0 & 8 & 0 & 4 & 80 \\
\hline 422 & 8 & 0 & 8 & 0 & 3 & 81 \\
\hline 202 & 8 & 0 & 7 & 2 & 6 & 77 \\
\hline 200 & 12 & 0 & 5 & 2 & 11 & 70 \\
\hline P1-1 & 8 & 4 & 8 & 0 & 5 & 75 \\
\hline P2-1 & 8 & 4 & 10 & 0 & 5 & 73 \\
\hline P2-2 & 8 & 5 & 11 & 0 & 5 & 71 \\
\hline P2-3 & 8 & 3 & 10 & 0 & 6 & 73 \\
\hline P2-4 & 8 & 5 & 11 & 0 & 6 & 70 \\
\hline P2-5 & 8 & 5 & 10 & 0 & 7 & 70 \\
\hline
\end{tabular}


WSRC-RP-2006-00002

Revision 0

\subsection{THE APPROACH AND CRITERIA FOR ACCEPTABILITY}

As stated in Section 2.0, the assessments conducted as part of this effort were strictly paper studies that were driven by predictions from glass property/glass composition models. However, the assessments do provide meaningful insight into the viability of sludge/frit glass systems since the models used in the assessment are the same as the models anticipated to be in DWPF's Product Composition Control System (PCCS) during the processing of SB4. The major property models included those for liquidus temperature $\left(\mathrm{T}_{\mathrm{L}}\right)$, viscosity $(\eta)$, and durability (as defined by the Product Consistency Test [PCT] [ASTM 2002] response in terms of the preliminary glass dissolution estimator $\left(\Delta \mathrm{G}_{\mathrm{P}}\right)$ (Jantzen et al. 1995)). It should be noted that the proposed durability limits by Edwards et al. (2003) and the new viscosity model developed by Jantzen (2005) were used in this assessment. ${ }^{2}$ Jantzen et al. (1995) and Brown et al. (2001) provide more details on the development of the PCCS models. In addition, the potential for the formation of a nepheline primary crystalline phase was also included in the assessment using a nepheline discriminator function described by Li et al. (2003). ${ }^{3}$ The nepheline discriminator model is currently not part of PCCS. However, based on recent experimental results (Peeler et al. 2005b and 2006), a strategy for controlling or limiting access to higher WLs in which nepheline formation can lead to detrimental effects on durability must be developed. Options for mitigating this potential include (but are not limited to) the implementation of the nepheline discriminator into PCCS or the use of an administrative control limit based on a fixed nepheline formation value.

There are two stages of investigation that may be used in the assessment of a candidate sludge: a Nominal Stage (a screening tool for the sludge that is typically applied to a large set of candidate frits such as that provided in Table 3-3) and a Variation Stage (a more thorough assessment of the sludge conducted for a select set of frits). These are discussed in Sections 4.2 and 4.3, respectively. For either stage, glass compositions were generated to represent combinations of the sludge/frit at waste loadings of interest. The acceptability of the model predictions for a particular glass composition for either stage was judged by employing the same criteria that are used by PCCS in its Measurement Acceptability Region (MAR) evaluations. Acceptable predicted properties for this assessment were based on satisfying their respective MAR limits. Brown, Postles, and Edwards (2002) provide a detailed discussion of how the MAR limits are utilized in PCCS. Thus, the value of the frit development effort in its assessment of a glass composition is that it mirrors the results that would be generated by the PCCS MAR for the same glass.

\section{1 $\mathrm{SO}_{4}{ }^{=}$Limitation}

In the MAR assessments of the sludge compositions that follow (both Nominal and Variation Stages), the $\mathrm{SO}_{4}{ }^{2}$ concentrations in glass will be calculated, but an assumed $\mathrm{SO}_{4}{ }^{=}$ limit will not be used to restrict the projected operating windows. Given there is no MAR

\footnotetext{
${ }^{2}$ It is assumed that the new durability limits and the new viscosity model will be implemented in DWPF to support SB4 processing. The durability limits remove excess conservatism in the current model without compromising product quality and provide access into higher alkali compositional regions which may improve melt rate and/or waste loading. The modifications to implement the new viscosity model in PCCS are described by Edwards and Peeler in the memorandum SRNL-SCS-2005-00054.

${ }^{3} \mathrm{Li}$ et al. (2003) indicate that sodium alumino-borosilicate glasses are prone to nepheline crystallization if their compositions projected on the $\mathrm{Na}_{2} \mathrm{O}-\mathrm{Al}_{2} \mathrm{O}_{3}-\mathrm{SiO}_{2}$ ternary fall within the nepheline primary phase field. In particular, durable glasses with $\mathrm{SiO}_{2} /\left(\mathrm{SiO}_{2}+\mathrm{Na}_{2} \mathrm{O}+\mathrm{Al}_{2} \mathrm{O}_{3}\right)>0.62$, where the oxides are expressed as mass fractions in the glass, do not precipitate nepheline as their primary phase.
} 
uncertainty associated with the $\mathrm{SO}_{4}{ }$ concentration, the maximum WL for each SB4 option can be determined as a function of an assumed $\mathrm{SO}_{4}{ }^{=}$solubility limit based strictly on mathematics (i.e., the assumed $\mathrm{SO}_{4}{ }^{=}$solubility limit divided by the $\mathrm{SO}_{4}{ }^{=}$concentration in sludge times 100). For example, if the $\mathrm{SO}_{4}{ }^{=}$concentration in sludge was $1.121 \mathrm{wt} \%$ (as it is for Case 8 and $8 \mathrm{a}$ Batch 1- see Table 3-1) and the assumed $\mathrm{SO}_{4}{ }{ }$ solubility limit was 0.4 $\mathrm{wt} \%$ (in glass), then the maximum $\mathrm{WL}$ achievable (based strictly on the $\mathrm{SO}_{4}{ }$ solubility limit) would be $\sim 35.7 \mathrm{wt} \%$. If the $\mathrm{SO}_{4}{ }{ }$ solubility limit were $0.5 \mathrm{wt} \%$ or $0.6 \mathrm{wt} \%$, then the maximum achievable WLs (based strictly on the $\mathrm{SO}_{4}{ }^{2}$ solubility limit) for this case would be $44.6 \%$ and $53.5 \%$, respectively. Although one can easily calculate the maximum WL for a given $\mathrm{SO}_{4}{ }^{=}$solubility limit, properties other than $\mathrm{SO}_{4}{ }^{=}$solubility may restrict the upper WL prior to the assumed $\mathrm{SO}_{4}{ }^{=}$limit - especially when a $0.6 \mathrm{wt} \%$ limit is assumed which Lorier et al. (2005) have suggested was feasible for SB4 glasses. Although a nominal $\mathrm{SO}_{4}{ }^{=}$ value has been added to each sludge option, an assumed $\mathrm{SO}_{4}{ }^{=}$solubility limit in PCCS was not activated during the model-based assessment. However, an assessment of the $\mathrm{SO}_{4}{ }^{=}$ limit and its potential impact on the upper WL attainable for select options is provided in Section 5.1.

\subsection{Overview of Nominal Assessment}

In general, the Nominal Stage evaluation provides a quick assessment of the viability of a particular sludge option relative to a potentially large set of candidate frits. Only the nominal composition of the sludge is used in this stage of the evaluation - no sludge variation is accounted for in this phase. Glass compositions are generated for a sludge/frit system at waste loadings of 25 to $60 \%$ in increments of 1 percentage point. This leads to the generation of 36 glass compositions for each sludge/frit combination. Each of these glasses is evaluated against the PCCS MAR and its acceptability is determined. The resulting evaluation identifies a subinterval within the 25 to $60 \%$ interval of WLs that defines the projected operating window in WLspace for the sludge/frit glass system. The evaluation also identifies the property model (e.g., viscosity or $\mathrm{T}_{\mathrm{L}}$ ) that limits the operating window when the window is restricted at either or both ends of the 25 to $60 \%$ interval. The Nominal Stage evaluation combined each of the nominal sludge options of Tables 3-1 and 3-2 with each frit (listed in Table 3-3) at WLs from 25 through $60 \%$ (in increments of $1 \mathrm{wt} \%$ ). The results from this assessment (i.e., the operating windows for each of these glass systems) are provided in Section 5.2.

\subsection{Overview of Variation Stage Assessment}

Since the Nominal Stage assessment does not account for any anticipated compositional variation in a sludge option, there is an increased risk with respect to processability or product quality if a decision were to be based solely on this assessment. The risk is lessened by the Variation Stage assessment, which is used to gain insight into the robustness of candidate frits with respect to sludge compositional variation. Of particular concern for SB4 is the potential impact of sludge variation on the operating windows of the glass system due to the nepheline constraint. As a result of this concern, an aggressive Variation Stage assessment was conducted for the Set 2 and 3 sludge options (see Table 3.2). ${ }^{4}$

\footnotetext{
${ }^{4}$ The term "aggressive" refers to a change in the traditional approach by which the Variation Stage has previously been performed. Previous assessments have typically used a "standard" $\pm 5 \%, 7.5 \%$ or $10 \%$ variation around the nominal sludge composition to define the minimum and maximum values for each oxide of interest. Although effective, applying a set percentage to both major and minor components results in a limited range over which the minor components are assessed. In the "aggressive approach" a different strategy was taken for the minor components - which is described in Section 4.3.
} 
Variation Stage assessments were not conducted on the compositions of Set 1 due to changes in the priorities of the work being conducted by the frit development team. As stated above, the results from the assessments that were completed and reviewed with CBU allowed them to make adjustments to their preparation plans that provided additional cases for consideration by the frit development team. The evaluation of these revised plans took precedent over any additional work on the compositions in Table 3-1 and Table 3-2 such as Variation Stage assessments for the Set 1 options.

Table 4-1 provides the framework for the Variation Stage assessments. These assessments replace the nominal value for each oxide in the sludge with an interval (defined by minimum (min) and maximum (max) values) of possible values. The top two rows indicate the types of variation that were introduced to determine the minimums and maximums: a variation of $\pm 7.5 \%$ of the nominal was placed around the nominal to determine the min's and max's for the major oxides (i.e., $\mathrm{Al}_{2} \mathrm{O}_{3}, \mathrm{Fe}_{2} \mathrm{O}_{3}$, and $\mathrm{Na}_{2} \mathrm{O}$ ) and $\mathrm{a} \pm 1 \mathrm{wt} \%$ variation was placed around the nominal value for each individually tracked minor oxide (i.e., $\mathrm{CaO}, \mathrm{MgO}, \mathrm{MnO}, \mathrm{NiO}, \mathrm{SiO}_{2}$, and $\mathrm{U}_{3} \mathrm{O}_{8}$ ) and for "Others". The "Others" term was used to allow for the inclusion of the minor oxides that were not tracked individually. The composition of the "Others" component of the sludge is provided (as weight percentages) in Table 4-2. This is seen as an aggressive approach in that the variation introduced for $\mathrm{CaO}, \mathrm{MgO}, \mathrm{MnO}, \mathrm{NiO}, \mathrm{SO}_{4}{ }^{\prime}, \mathrm{SiO}_{2}, \mathrm{U}_{3} \mathrm{O}_{8}$, and "Others" is very large (when expressed as a percentage of the nominal values) as compared to the variation for the major oxides of $\mathrm{Al}_{2} \mathrm{O}_{3}, \mathrm{Fe}_{2} \mathrm{O}_{3}$, and $\mathrm{Na}_{2} \mathrm{O}$. The variation applied to the nominal $\mathrm{SO}_{4}{ }^{=}$was $\pm 0.25 \mathrm{wt} \%$. Also note that the rows of Tables 4-1 and 4-2 show that the Variation Stage assessments were conducted only for the sludge options corresponding to Case 15 and Case $7 \mathrm{~b}$ as indicated above. 
Table 4-1. Developing Bounding Regions for Case 15 and Case $7 \mathrm{~b}$ (as mass fractions)

\begin{tabular}{|c|c|c|c|c|c|c|c|c|c|c|c|c|}
\hline Variation (+/-) & & 7.5 & 1 & 7.5 & 1 & 1 & 7.5 & 1 & 0.25 & 1 & 1 & 1 \\
\hline Unit of Measure & & $\%$ & wt $\%$ & $\%$ & wt $\%$ & wt\% & $\%$ & wt $\%$ & wt $\%$ & wt $\%$ & wt $\%$ & wt $\%$ \\
\hline Option & & $\overline{\mathrm{Al}_{2} \mathrm{O}_{3}}$ & $\mathrm{CaO}$ & $\mathrm{Fe}_{2} \mathrm{O}_{3}$ & MgO & MnO & $\mathrm{Na}_{2} \mathrm{O}$ & NiO & $\mathrm{SO}_{4}{ }^{=}$ & $\mathrm{SiO}_{2}$ & $\mathrm{U}_{3} \mathrm{O}_{8}$ & Others \\
\hline \multirow[t]{2}{*}{ Case 15 Batch 1} & $\min$ & 0.40945 & 0.00660 & 0.16535 & $-0.00209^{5}$ & 0.02845 & 0.16289 & 0.00390 & 0.01014 & 0.05335 & 0.02599 & 0.00366 \\
\hline & $\max$ & 0.47585 & 0.02660 & 0.19217 & 0.01791 & 0.04845 & 0.18931 & 0.02390 & 0.01514 & 0.07335 & 0.04599 & 0.02366 \\
\hline \multirow[t]{2}{*}{ Case 15 Blend 1} & $\min$ & 0.23460 & 0.01441 & 0.25125 & 0.01551 & 0.04603 & 0.18952 & 0.00612 & 0.00960 & 0.03205 & 0.06812 & 0.00552 \\
\hline & $\max$ & 0.27264 & 0.03441 & 0.29199 & 0.03551 & 0.06603 & 0.22026 & 0.02612 & 0.01460 & 0.05205 & 0.08812 & 0.02552 \\
\hline \multirow[t]{2}{*}{ Case 15 Batch 2} & $\min$ & 0.26354 & 0.00663 & 0.18561 & 0.00458 & 0.04103 & 0.22917 & 0.02888 & 0.00421 & 0.03209 & 0.07063 & 0.00612 \\
\hline & $\max$ & 0.30628 & 0.02663 & 0.21571 & 0.02458 & 0.06103 & 0.26633 & 0.04888 & 0.00921 & 0.05209 & 0.09063 & 0.02612 \\
\hline \multirow[t]{2}{*}{ Case 15 Blend 2} & $\min$ & 0.25627 & 0.00814 & 0.19814 & 0.00675 & 0.04180 & 0.22453 & 0.02401 & 0.00528 & 0.03187 & 0.06971 & 0.00593 \\
\hline & $\max$ & 0.29783 & 0.02814 & 0.23028 & 0.02675 & 0.06180 & 0.26095 & 0.04401 & 0.01028 & 0.05187 & 0.08971 & 0.02593 \\
\hline \multirow[t]{2}{*}{ Case 7b Batch 1} & $\min$ & 0.28559 & 0.00571 & 0.17027 & 0.00211 & 0.03506 & 0.24758 & 0.02046 & 0.00899 & 0.03510 & 0.05498 & 0.00462 \\
\hline & $\max$ & 0.33190 & 0.02571 & 0.19789 & 0.02211 & 0.05506 & 0.28773 & 0.04046 & 0.01399 & 0.05510 & 0.07498 & 0.02462 \\
\hline \multirow[t]{2}{*}{ Case $7 \mathrm{~b}$ Blend 1} & $\min$ & 0.19250 & 0.01396 & 0.25163 & 0.01675 & 0.04814 & 0.21988 & 0.01211 & 0.00911 & 0.02578 & 0.07796 & 0.00584 \\
\hline & $\max$ & 0.22371 & 0.03396 & 0.29244 & 0.03675 & 0.06814 & 0.25554 & 0.03211 & 0.01411 & 0.04578 & 0.09796 & 0.02584 \\
\hline \multirow[t]{2}{*}{ Case $7 b$ Batch 2} & $\min$ & 0.30463 & 0.00675 & 0.18163 & 0.00291 & 0.03806 & 0.20512 & 0.02250 & 0.00683 & 0.03810 & 0.05931 & 0.00559 \\
\hline & $\max$ & 0.35403 & 0.02675 & 0.21108 & 0.02291 & 0.05806 & 0.23838 & 0.04250 & 0.01183 & 0.05810 & 0.07931 & 0.02559 \\
\hline \multirow[t]{2}{*}{ Case $7 \mathrm{~b}$ Blend 2} & $\min$ & 0.27855 & 0.00825 & 0.19608 & 0.00588 & 0.04003 & 0.21193 & 0.02006 & 0.00725 & 0.03517 & 0.06306 & 0.00557 \\
\hline & $\max$ & 0.32372 & 0.02825 & 0.22788 & 0.02588 & 0.06003 & 0.24630 & 0.04006 & 0.01225 & 0.05517 & 0.08306 & 0.02557 \\
\hline
\end{tabular}

\footnotetext{
${ }^{5}$ The $1 \mathrm{wt} \%$ variation around the nominal mass fraction concentration of 0.00791 for the Case 15 Batch $1 \mathrm{MgO}$ drives the lower limit of the concentration for this oxide negative; a value of zero was used for this lower limit in the development of the EVs for Case 15 Batch 1.
} 
Table 4-2. Components (as weight percentages) of Others for Case 15 and Case 7b

\begin{tabular}{|c|c|c|c|c|c|c|c|c|c|c|c|c|}
\hline Option & BaO & $\mathrm{Ce}_{2} \mathrm{O}_{3}$ & $\mathrm{Cr}_{2} \mathrm{O}_{3}$ & $\mathrm{CuO}$ & $\mathbf{K}_{2} \mathbf{O}$ & $\mathrm{La}_{2} \mathbf{O}_{3}$ & PbO & $\mathrm{ThO}_{2}$ & $\mathrm{TiO}_{2}$ & ZnO & $\mathrm{ZrO}_{2}$ & Others \\
\hline Case 15 Batch 1 & 7.10 & 0.00 & 14.06 & 0.00 & 45.31 & 7.91 & 0.00 & 9.52 & 1.83 & 0.00 & 14.28 & 100.00 \\
\hline Case 15 Blend 1 & 8.31 & 9.86 & 13.98 & 3.93 & 22.68 & 7.15 & 5.99 & 4.32 & 1.74 & 6.44 & 15.59 & 100.00 \\
\hline Case 15 Batch 2 & 9.24 & 3.97 & 13.34 & 2.17 & 25.50 & 5.83 & 2.17 & 4.65 & 15.01 & 3.54 & 14.58 & 100.00 \\
\hline Case 15 Blend 2 & 9.04 & 5.15 & 13.50 & 2.51 & 24.92 & 6.09 & 2.95 & 4.58 & 12.37 & 4.14 & 14.75 & 100.00 \\
\hline Case 7b Batch 1 & 8.75 & 3.06 & 13.51 & 1.64 & 30.14 & 6.32 & 1.68 & 5.77 & 11.91 & 2.71 & 14.50 & 100.00 \\
\hline Case $7 \mathrm{~b}$ Blend 1 & 8.81 & 10.53 & 13.80 & 4.35 & 18.39 & 6.64 & 6.31 & 3.28 & 5.15 & 7.11 & 15.65 & 100.00 \\
\hline Case 7b Batch 2 & 8.75 & 3.06 & 13.51 & 1.64 & 30.14 & 6.32 & 1.68 & 5.77 & 11.91 & 2.71 & 14.50 & 100.00 \\
\hline Case $7 \mathrm{~b}$ Blend 2 & 8.76 & 4.72 & 13.57 & 2.24 & 27.52 & 6.39 & 2.71 & 5.22 & 10.41 & 3.69 & 14.76 & 100.00 \\
\hline
\end{tabular}


Consider one of the sludge options of Table 4-1. A sludge composition is in the region corresponding to this option if its concentration for each oxide is within the min and max interval for that oxide and the sum of the concentrations equals 1 . Such a composition is a mixture of oxides at concentrations that correspond to one of the possible compositions for that sludge option as defined by Table 4-1. Algorithms are available in statistical software packages such as JMP (SAS 2002) to generate the compositions that bound the region defined by Table 4-1 for a particular sludge option - the bounding compositions are the extreme vertices (or "corner points") of the region.

JMP Version 5.1.2 (SAS 2002) was used to generate the extreme vertices (EVs) or corner points of the sludge regions defined by Table 4-1 for the options of Case 15 and Case 7B. The centroid (or average of the EVs) for each region was also generated as it was tracked during the Variation Stage to ensure consistent results with the Nominal Stage assessment. For each sludge option, candidate frits were selected to be combined with the EVs generated by JMP at waste loadings from 25 to $60 \%$. The resulting glass compositions were evaluated against the PCCS MAR criteria to determine their respective acceptability. A candidate frit is considered to demonstrate robustness to the variation in a sludge option if $100 \%$ of the EVs for that option meet the PCCS MAR criteria over a wide sub-interval of the 25 to $60 \%$ WL interval. In addition, where less than $100 \%$ of the EVs are acceptable, identifying the constraint or constraints that are not met (i.e., the constraint(s) that limit the operating window) is of interest.

Identification or selection of the candidate frits to be used in the Variation Stage assessment was primarily based on the results of the Nominal Stage assessments. Table 4-3 provides an overview of the glass systems that were evaluated as part of the Variation Stage. The number of EVs for each sludge option is also provided in Table 4-3. The results from this assessment (i.e., the operating windows for each of these glass systems) are provided in Section 5.3.

Table 4-3. Glass Systems Evaluated During Variation Stage Assessments

\begin{tabular}{||c|c|c|c||}
\hline Case & Sludge Option & Number of EVs & Candidate Frits \\
\hline 15 & Batch 1 & 2144 & 320 \\
\hline 15 & Blend 1 & 2464 & 418,425, P3-1 \\
\hline 15 & Batch 2 & 2534 & P3-1 \\
\hline 15 & Blend 2 & 2464 & 418,425, P3-1 \\
\hline 7B & Batch 1 & 2492 & 418, P3-1 \\
\hline 7B & Blend 1 & 2464 & 418,425, P3-1 \\
\hline 7B & Batch 2 & 2464 & 418, P3-1 \\
\hline 7B & Blend 2 & 2534 & 418,425, P3-1 \\
\hline
\end{tabular}




\subsection{PROJECTED OPERATING WINDOWS FOR THE SB4 OPTIONS}

In this section, the projected operating windows are presented for the Nominal and Variation Stage assessments. Prior to the discussion of these results, the impact of the $\mathrm{SO}_{4}{ }^{=}$solubility limit on the operating windows is discussed.

\subsection{Impact of $\mathrm{SO}_{4}=$}

The nominal $\mathrm{SO}_{4}{ }^{=}$concentration for SB4 is dependent on three primary inputs: (1) the wash endpoint for SB4, (2) the SB3 heel volume, and (3) the inclusion (or exclusion) of ARP streams. ARP is not included in the scope of this effort. From a historical SB4 perspective, Lorier et al. (2005) provided preliminary results that indicated that the $0.6 \mathrm{wt} \% \mathrm{SO}_{4}{ }$ solubility limit (defined for SB3) was applicable for select SB4 sludge (based on Lilliston 2005) - frit combinations. No salt layer formation was evident in any test $(30 \%$ or $40 \% \mathrm{WL})$ with Frit 320 until $\mathrm{SO}_{4}{ }{ }$ concentrations of $0.8 \mathrm{wt} \%$ were targeted. Tests with Frit 418 showed that $\mathrm{SO}_{4}{ }^{=}$was apparent on the glass surface of tests at $40 \% \mathrm{WL}$ and $0.6 \mathrm{wt} \% \mathrm{SO}_{4}{ }^{=}$- indicating that a $\mathrm{SO}_{4}{ }^{=}$limit of $0.5 \mathrm{wt} \%$ may be required. The results suggested that the $\mathrm{SO}_{4}{ }^{=}$solubility limit was dependent on the overall glass composition - in general, the more refractory the frit, the lower the $\mathrm{SO}_{4}{ }^{=}$ solubility.

Given Frit 320 and Frit 418 are somewhat bounding in terms of their $\mathrm{Na}_{2} \mathrm{O}$ concentrations (for the "sliding $\mathrm{Na}_{2} \mathrm{O}$ scale" series - see Table 3-3) and acknowledging the recent results from Lorier et al. (2005), an assessment of the maximum WLs that could be achieved prior to a specific system becoming $\mathrm{SO}_{4}{ }^{=}$limited at either 0.5 and $0.6 \mathrm{wt} \%$ is warranted. It should be noted that a formal experimental assessment of $\mathrm{SO}_{4}{ }^{=}$solubility based on the final SB4 preparation plan and candidate frit compositions will be addressed in a subsequent report.

Table 5-1 shows how much $\mathrm{SO}_{4}{ }^{=}$must be retained (assuming no volatilization occurs) in the glass at various WLs (by increments of 5 percentage points). Since there is no measurement uncertainty accounted for in the $\mathrm{SO}_{4}{ }^{=}$PCCS constraint, this table can be used as a basis for understanding how $\mathrm{SO}_{4}{ }^{=}$ solubility might limit DWPF's operating window (i.e., targeted WL) depending upon its solubility limit in the glass system. The cells highlighted in yellow indicate that the solubility limit would be exceeded assuming a $0.5 \mathrm{wt} \%$ limit in PCCS. Those cells highlighted in green represent conditions in which the solubility limit would be exceeded assuming a $0.6 \mathrm{wt} \%$ value. The mathematical WL limit at which the assumed solubility limit would be exceeded can easily be calculated (assuming no volatilization occurs).

Assuming a $0.6 \mathrm{wt} \% \mathrm{SO}_{4}{ }^{=}$limit, Case 15B (both Batch 1 and Blend 1) and Case 15 (both Batch 1 and Blend 1) are the only sludge options that would exceed this limit at WLs at or below $50 \%$. Case $15 \mathrm{~B}$ Batch 1 being the "worst case" scenario as WLs would be limited to $~ 36 \%$ WL or lower. It should be noted that the Batch 1 composition reflects a qualification sample and thus the targeted WL may not be as critical. When considering the "blends" (i.e., sludges to be processed in DWPF), the maximum WLs for the Case $15 \mathrm{~B}$ Blend 1 and Case 15 Blend 1 would be $\sim 48 \%$ and $49 \%$ WL, respectively. The potential for the $0.6 \mathrm{wt} \% \mathrm{SO}_{4}{ }^{=}$solubility limit to constrain DWPF processing is minimal as another constraint will likely restrict assess to these higher WLs.

Obviously, if one assumes a lower $\mathrm{SO}_{4}{ }$ solubility limit (i.e., $0.5 \mathrm{wt} \%$ ), the impact on select systems is more severe (especially the Blend 1 and Batch 1 sludges for Case 15 and Case 15B). In general, WLs of at least $\sim 40-45 \%$ would be obtainable for most systems of interest. One interesting observation is the fact that none of the Blend 2 options (which would be processed in DWPF) are limited by $\mathrm{SO}_{4}{ }^{=}$even with the lower assumed $0.5 \mathrm{wt} \%$ limit. As previously mentioned, although these data provide insight into the 
possible impact of $\mathrm{SO}_{4}{ }^{=}$solubility limits on the projected operating window, a formal experimental assessment of $\mathrm{SO}_{4}{ }^{=}$solubility based on the final SB4 preparation plan and candidate frit compositions will be addressed in a subsequent report.

Table 5-1. $\mathrm{SO}_{4}{ }^{=}$Concentrations in the Glass for the Sludge Cases at Select WLs

\begin{tabular}{|c|c|c|c|c|c|c|c|}
\hline \multirow{2}{*}{ Set } & \multirow{2}{*}{ Sludge Options } & \multirow{2}{*}{$\mathrm{SO}_{4}=\mathrm{wt} \%$} & \multicolumn{5}{|c|}{ 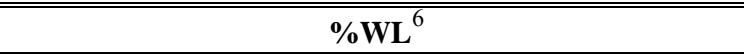 } \\
\hline & & & 30 & 35 & 40 & 45 & 50 \\
\hline 1 & Case 8 and $8 \mathrm{a}-$ Batch 1 & 1.121 & 0.336 & 0.392 & 0.448 & 0.504 & 0.561 \\
\hline 1 & Case 8 - Blend 1 & 1.138 & 0.341 & 0.398 & 0.455 & 0.512 & 0.569 \\
\hline 1 & Case $8 \mathrm{~A}$ - Blend 1 & 1.124 & 0.337 & 0.393 & 0.450 & 0.506 & 0.562 \\
\hline 1 & Case 7 Batch 1 & 0.965 & 0.290 & 0.338 & 0.386 & 0.434 & 0.483 \\
\hline 1 & Case 7 Blend 1 & 1.124 & 0.337 & 0.393 & 0.450 & 0.506 & 0.562 \\
\hline 1 & Case 7 Batch 2 & 0.766 & 0.230 & 0.268 & 0.306 & 0.345 & 0.383 \\
\hline 1 & Case 7 Blend 2 & 0.833 & 0.250 & 0.292 & 0.333 & 0.375 & 0.417 \\
\hline 1 & Case 15B - Batch 1 & 1.649 & 0.495 & 0.577 & 0.660 & 0.742 & 0.825 \\
\hline 1 & Case 15B - Blend 1 & 1.246 & 0.374 & 0.436 & 0.498 & 0.561 & 0.623 \\
\hline 1 & Case 15B - Batch 2 & 0.867 & 0.260 & 0.303 & 0.347 & 0.390 & 0.434 \\
\hline 1 & Case $15 \mathrm{~B}-$ Blend 2 & 0.934 & 0.280 & 0.327 & 0.374 & 0.420 & 0.467 \\
\hline 1 & Case 16 - Batch 1 & 0.302 & 0.091 & 0.106 & 0.121 & 0.136 & 0.151 \\
\hline 1 & Case 16 - Blend 1 & 0.725 & 0.218 & 0.254 & 0.290 & 0.326 & 0.363 \\
\hline 1 & Case 16 - Batch 2 & 1.174 & 0.352 & 0.411 & 0.470 & 0.528 & 0.587 \\
\hline 1 & Case $16-$ Blend 2 & 1.060 & 0.318 & 0.371 & 0.424 & 0.477 & 0.530 \\
\hline 2 & Case 15 Batch 1 & 1.264 & 0.379 & 0.442 & 0.506 & 0.569 & 0.632 \\
\hline 2 & Case 15 Blend 1 & 1.210 & 0.363 & 0.424 & 0.484 & 0.545 & 0.605 \\
\hline 2 & Case 15 Batch 2 & 0.671 & 0.201 & 0.235 & 0.268 & 0.302 & 0.336 \\
\hline 2 & Case 15 Blend 2 & 0.778 & 0.233 & 0.272 & 0.311 & 0.350 & 0.389 \\
\hline 3 & Case $7 \mathrm{~b}$ Batch 1 & 1.149 & 0.345 & 0.402 & 0.460 & 0.517 & 0.575 \\
\hline 3 & Case $7 \mathrm{~b}$ Blend 1 & 1.161 & 0.348 & 0.406 & 0.464 & 0.522 & 0.581 \\
\hline 3 & Case $7 \mathrm{~b}$ Batch 2 & 0.933 & 0.280 & 0.327 & 0.373 & 0.420 & 0.467 \\
\hline 3 & Case $7 \mathrm{~b}$ Blend 2 & 0.975 & 0.293 & 0.341 & 0.390 & 0.439 & 0.488 \\
\hline
\end{tabular}

\subsection{Results from the Nominal Stage Assessments}

Exhibit A1 in the Appendix provides the details of the MAR and nepheline results at each WL for each sludge/frit glass system. Table 5-2 provides (in two parts) ${ }^{7}$ the projected operating windows (defined in terms of minimum and maximum WLs) for each glass system based upon the MAR assessments and an activated nepheline constraint. It should be noted that a "_. in Table 5-2 indicates that there is no operating window for that frit - sludge combination based on the acceptance criteria used.

Obviously, numerous comparisons can be made among the SB4 scenarios for a specific frit, and, yet, even more when considering differences among the frits for the sludge options. Be that as it may, the authors have elected to provide a detailed assessment of one of the sludge options -

\footnotetext{
${ }^{6}$ The cells highlighted in yellow indicate that the solubility limit would be exceeded assuming a $0.5 \mathrm{wt} \%$ limit in PCCS. Those cells highlighted in green represent conditions in which the solubility limit would be exceeded assuming a $0.6 \mathrm{wt} \%$ value.

${ }^{7}$ The two parts are differentiated by the $\mathrm{Na}_{2} \mathrm{O}$ concentration in frit. Part 1 shows the frits containing $3-6 \mathrm{wt} \% \mathrm{Na}_{2} \mathrm{O}$; Part 2 contains the frits with $7-15$ wt $\% \mathrm{Na}_{2} \mathrm{O}$.
} 
specifically Frit 418 with Case 15 (see the yellow highlighted cells of part 2 in Table 5-2 and, for details, of part 3 of Exhibit A1 ). Frit 418 was selected given it is the current frit being used to process SB3 and a primary candidate for SB4 as well. Case 15 was selected given it was one of the sludge composition used in the limited Variation Stage assessment and will provide continuity in the transition from the discussion of the Nominal Stage results to the discussion of the Variation Stage results. The details associated with the Nominal Stage assessment of these cases are highlighted in Appendix A. The discussion of the Frit 418 - Case 15 flowsheet will provide a general understanding of how the table can be interpreted and applied to other systems that may be of interest to the reader.

For the Case 15 Batch 1 option, there is no projected operating window when Frit 418 is utilized. From Appendix A, predictions of high viscosity limit access to WLs of $25 \%$ to $39 \%$ with an additional constraint (nepheline formation potential) also becoming an issue at $40 \%$ and higher. Although Frit 418 could not be used to qualify this batch, other frits are available with operating windows (e.g., 425, 417, 320, 431, P2-2, and P2-4). Operating windows do exist for these alternative frits, but the maximum WL is typically in the low-to-mid-30's with nepheline predictions becoming the primary issue. Therefore, qualification of this batch would most likely be based on a relatively low WL (high 20's to low 30's) and possibly with a different frit than used to process the "blend" in DWPF. It should be noted that the $\mathrm{Fe}_{2} \mathrm{O}_{3}$-based frits (P2-2 and $\left.\mathrm{P} 2-4\right)$ provide operating windows of $25-38$ and $39 \%$, respectively, given the suppression of nepheline formation to higher WLs for these glass systems.

Coupling Frit 418 with Batch 2 (another qualification composition), yields a projected operating window of $25-43 \%$ WL. From Appendix A, nepheline becomes the limiting factor at $44 \% \mathrm{WL}$ and higher. This sludge could be qualified with Frit 418 at reasonable WLs of interest.

When Frit 418 is used with Case 15 Blend 1 and Blend 2, the projected operating windows are 25 $46 \%$ and $25-43 \% \mathrm{WL}$, respectively. Predictions of $\mathrm{T}_{\mathrm{L}}$ limit access to WLs of $47 \%$ and higher in the Blend 1 system. Nepheline concerns limit access to WLs of $44 \%$ and higher in the Blend 2 system. With respect to DWPF processing, Frit 418 could be used with both Blend 1 and Blend 2 with a potential need to reduce the targeted WL for Blend 2. This latter statement assumes that the maximum waste throughput for Blend 1 would be achieved at WLs of $44 \%-46 \%$. Although historically maximum waste throughput has not been achieved at the maximum WL identified through the model based assessments (but at some intermediate WL), predictions of $T_{L}$ would restrict WLs to $43 \%$ or lower for the Blend 2 system even if the higher WLs were realized for Blend 1.

Again, the number of comparisons within a specific flowsheet or among different flowsheets could be overwhelming. The summary in Table 5-2 coupled with the details in Appendix A provide a technical basis from which other comparisons can be made that may be of interest to the reader. Given the preferred preparation plan as defined by the CBU is not shown in this report, the discussions provided in this report have been held to a minimum. 
Table 5-2. Operating Windows from Nominal Assessments Expressed as WL Intervals (part 1)

Constraint not met: neph (nepheline), TL (liquidus temperature), lvisc (low viscosity - new model), hvisc (high viscosity - new model), and Del GP - durability.

\begin{tabular}{|c|c|c|c|c|c|c|c|c|c|c|c|c|c|c|}
\hline & $\mathrm{Na}_{2} \mathrm{O}(\mathrm{wt} \%)$ content & 3 & 4 & 5 & 5 & 5 & 5 & 5 & 5 & 6 & 6 & 6 & 6 & 6 \\
\hline Set & Frit & 422 & d1-1 & 473 & $\mathrm{P} 1-1$ & P2-1 & $\mathrm{P} 2-2$ & P3-1 & $\mathrm{t} 1-1$ & 202 & 432 & P2-3 & $\mathrm{P} 2-4$ & P3-2 \\
\hline 1 & Case 15B - Batch 1 & -8 & - & - & - & $\begin{array}{c}25-39 \\
\text { neph }\end{array}$ & $\begin{array}{c}\begin{array}{c}25-38 \\
\text { neph }\end{array} \\
\end{array}$ & $\begin{array}{c}25-40 \\
\text { neph }\end{array}$ & - & - & - & $\begin{array}{c}25-39 \\
\text { neph }\end{array}$ & $\begin{array}{c}25-37 \\
\text { neph }\end{array}$ & $\begin{array}{c}25-39 \\
\text { neph }\end{array}$ \\
\hline 1 & Case 15B - Batch 2 & - & - & - & - & $\begin{array}{c}25-37 \\
\text { TL }\end{array}$ & $\begin{array}{c}25-37 \\
\text { TL }\end{array}$ & $\begin{array}{c}25-42 \\
\text { TL }\end{array}$ & - & - & - & $\begin{array}{c}25-39 \\
\text { TL }\end{array}$ & $\begin{array}{c}25-38 \\
\text { TL }\end{array}$ & $\begin{array}{c}25-41 \\
\text { TL }\end{array}$ \\
\hline 1 & Case 15B - Blend 1 & $\begin{array}{c}\text { hvisc } \\
34-41 \\
\text { TL } \\
\end{array}$ & $\begin{array}{c}\text { hvisc } \\
31-43 \\
\text { TL } \\
\end{array}$ & $\begin{array}{c}\text { hvisc } \\
27-44 \\
\text { TL } \\
\end{array}$ & $\begin{array}{c}25-39 \\
\text { TL }\end{array}$ & $\begin{array}{l}25-38 \\
\text { lvisc }\end{array}$ & $\begin{array}{c}25-31 \\
\text { lvisc }\end{array}$ & $\begin{array}{l}25-41 \\
\text { lvisc }\end{array}$ & $\begin{array}{c}25-42 \\
\text { TL }\end{array}$ & $\begin{array}{c}\text { hvisc } \\
28-40 \\
\text { TL } \\
\end{array}$ & $\begin{array}{c}25-45 \\
\text { TL }\end{array}$ & $\begin{array}{l}25-37 \\
\text { lvisc }\end{array}$ & $\begin{array}{l}25-27 \\
\text { lvisc }\end{array}$ & $\begin{array}{l}25-41 \\
\text { lvisc }\end{array}$ \\
\hline 1 & Case 15B - Blend 2 & - & - & - & $\begin{array}{c}\text { hvisc } \\
31-35 \\
\text { TL }\end{array}$ & $\begin{array}{c}25-38 \\
\text { TL }\end{array}$ & $\begin{array}{c}25-38 \\
\text { TL }\end{array}$ & $\begin{array}{c}25-43 \\
\text { TL }\end{array}$ & $\begin{array}{c}\text { hvisc } \\
38-38 \\
\text { TL }\end{array}$ & - & $\begin{array}{c}\text { hvisc } \\
39-41 \\
\text { TL }\end{array}$ & $\begin{array}{c}25-40 \\
\text { TL }\end{array}$ & $\begin{array}{c}25-39 \\
\text { TL }\end{array}$ & $\begin{array}{c}25-42 \\
\text { TL }\end{array}$ \\
\hline 1 & Case 16 - Batch 1 & - & - & - & - & - & - & - & - & - & - & - & - & - \\
\hline 1 & Case 16 - Batch 2 & - & - & - & - & $\begin{array}{c}25-41 \\
\text { neph }\end{array}$ & $\begin{array}{c}25-40 \\
\text { neph }\end{array}$ & $\begin{array}{c}25-42 \\
\text { neph }\end{array}$ & - & - & - & $\begin{array}{c}25-40 \\
\text { neph }\end{array}$ & $\begin{array}{c}25-39 \\
\text { neph }\end{array}$ & $\begin{array}{l}25-41 \\
\text { neph }\end{array}$ \\
\hline 1 & Case 16 - Blend 1 & $\begin{array}{c}\text { hvisc } \\
32-35 \\
\text { TL }\end{array}$ & $\begin{array}{c}\text { hvisc } \\
29-36 \\
\text { TL }\end{array}$ & $\begin{array}{c}\text { hvisc } \\
26-38 \\
\text { TL }\end{array}$ & $\begin{array}{c}25-34 \\
\text { TL }\end{array}$ & $\begin{array}{c}25-36 \\
\text { TL, lvisc }\end{array}$ & $\begin{array}{c}25-29 \\
\text { lvisc }\end{array}$ & $\begin{array}{c}25-39 \\
\text { lvisc }\end{array}$ & $\begin{array}{c}25-36 \\
\text { TL }\end{array}$ & $\begin{array}{c}\text { hvisc } \\
26-34 \\
\text { TL }\end{array}$ & $\begin{array}{c}25-39 \\
\text { TL }\end{array}$ & $\begin{array}{l}25-35 \\
\text { lvisc }\end{array}$ & $\begin{array}{l}25-26 \\
\text { lvisc }\end{array}$ & $\begin{array}{l}25-39 \\
\text { lvisc }\end{array}$ \\
\hline 1 & Case 16 - Blend 2 & - & - & $\begin{array}{c}\text { hvisc } \\
44-44 \\
\text { TL } \\
\end{array}$ & $\begin{array}{c}\text { hvisc } \\
32-39 \\
\text { TL } \\
\end{array}$ & $\begin{array}{c}25-42 \\
\text { TL }\end{array}$ & $\begin{array}{c}25-41 \\
\text { TL }\end{array}$ & $\begin{array}{c}25-44 \\
\text { neph }\end{array}$ & $\begin{array}{c}\text { lvisc } \\
39-42 \\
\text { TL } \\
\end{array}$ & - & $\begin{array}{c}\text { hvisc } \\
39-44 \\
\text { neph } \\
\end{array}$ & $\begin{array}{c}25-43 \\
\text { neph }\end{array}$ & $\begin{array}{c}25-41 \\
\text { neph }\end{array}$ & $\begin{array}{c}25-43 \\
\text { neph }\end{array}$ \\
\hline 1 & Case 7 Batch 1 & - & $\begin{array}{c}\text { hvisc } \\
45-45 \\
\text { neph }\end{array}$ & $\begin{array}{c}\text { hvisc } \\
41-44 \\
\text { neph }\end{array}$ & $\begin{array}{c}\text { hvisc } \\
29-42 \\
\text { TL }\end{array}$ & $\begin{array}{c}25-42 \\
\text { neph }\end{array}$ & $\begin{array}{c}25-41 \\
\text { neph }\end{array}$ & $\begin{array}{c}25-43 \\
\text { neph }\end{array}$ & $\begin{array}{c}\text { hvisc } \\
36-44 \\
\text { TL, neph }\end{array}$ & $\begin{array}{c}\text { hvisc } \\
42-43 \\
\text { TL, neph }\end{array}$ & $\begin{array}{c}\text { hvisc } \\
36-43 \\
\text { neph }\end{array}$ & $\begin{array}{c}25-41 \\
\text { neph }\end{array}$ & $\begin{array}{c}25-40 \\
\text { neph }\end{array}$ & $\begin{array}{c}25-42 \\
\text { neph }\end{array}$ \\
\hline 1 & Case 7 Batch 2 & - & - & - & - & $\begin{array}{c}25-37 \\
\mathrm{TL}\end{array}$ & $\begin{array}{c}25-37 \\
\text { TL }\end{array}$ & $\begin{array}{c}25-42 \\
\text { TL }\end{array}$ & - & - & - & $\begin{array}{c}25-39 \\
\text { TL } \\
\end{array}$ & $\begin{array}{c}25-38 \\
\text { TL } \\
\end{array}$ & $\begin{array}{c}25-41 \\
\text { TL }\end{array}$ \\
\hline 1 & Case 7 Blend 1 & $\begin{array}{c}\text { hvisc } \\
33-41 \\
\text { TL } \\
\end{array}$ & $\begin{array}{c}\text { hvisc } \\
30-42 \\
\text { TL } \\
\end{array}$ & $\begin{array}{c}\text { hvisc } \\
27-43 \\
\text { TL } \\
\end{array}$ & $\begin{array}{l}25-39 \\
\text { lvisc }\end{array}$ & $\begin{array}{l}25-38 \\
\text { lvisc }\end{array}$ & $\begin{array}{l}25-30 \\
\text { lvisc }\end{array}$ & $\begin{array}{l}25-40 \\
\text { lvisc }\end{array}$ & $\begin{array}{c}25-41 \\
\text { TL }\end{array}$ & $\begin{array}{c}\text { hvisc } \\
27-40 \\
\text { TL } \\
\end{array}$ & $\begin{array}{c}25-45 \\
\text { TL }\end{array}$ & $\begin{array}{l}25-37 \\
\text { lvisc }\end{array}$ & $\begin{array}{l}25-27 \\
\text { lvisc }\end{array}$ & $\begin{array}{l}25-40 \\
\text { lvisc }\end{array}$ \\
\hline 1 & Case 7 Blend 2 & - & - & - & $\begin{array}{c}\text { hvisc } \\
33-35 \\
\text { TL } \\
\end{array}$ & $\begin{array}{c}25-38 \\
\text { TL }\end{array}$ & $\begin{array}{c}25-38 \\
\text { TL }\end{array}$ & $\begin{array}{c}25-43 \\
\text { TL }\end{array}$ & - & - & $\begin{array}{c}\text { hvisc } \\
41-42 \\
\text { TL } \\
\end{array}$ & $\begin{array}{c}25-40 \\
\text { TL }\end{array}$ & $\begin{array}{c}25-39 \\
\text { TL }\end{array}$ & $\begin{array}{c}25-42 \\
\text { TL }\end{array}$ \\
\hline 1 & Case 8 - Blend 1 & $\begin{array}{c}\text { hvisc } \\
37-40 \\
\text { TL }\end{array}$ & $\begin{array}{c}\text { hvisc } \\
34-41 \\
\text { TL }\end{array}$ & $\begin{array}{c}\text { hvisc } \\
30-42 \\
\text { TL }\end{array}$ & $\begin{array}{c}25-38 \\
\text { TL }\end{array}$ & $\begin{array}{c}25-40 \\
\text { TL }\end{array}$ & $\begin{array}{l}25-35 \\
\text { lvisc }\end{array}$ & $\begin{array}{c}25-45 \\
\text { TL, lvisc }\end{array}$ & $\begin{array}{c}\text { hvisc } \\
26-40 \\
\mathrm{Tl}\end{array}$ & $\begin{array}{c}\text { hvisc } \\
31-38 \\
\text { TL }\end{array}$ & $\begin{array}{c}\text { hvisc } \\
26-43 \\
\text { TL }\end{array}$ & $\begin{array}{l}25-41 \\
\text { lvisc }\end{array}$ & $\begin{array}{c}25-31 \\
\text { lvisc }\end{array}$ & $\begin{array}{c}25-45 \\
T L, \text { lvisc }\end{array}$ \\
\hline
\end{tabular}

${ }^{8}$ A “-“ entry in a cell indicates no operating window for the corresponding glass system. 
Table 5-2. Operating Windows from Nominal Assessments Expressed as WL Intervals (part 1)

Constraint not met: neph (nepheline), TL (liquidus temperature), lvisc (low viscosity - new model), hvisc (high viscosity - new model), and Del GP - durability.

\begin{tabular}{|c|c|c|c|c|c|c|c|c|c|c|c|c|c|c|}
\hline & $\mathrm{Na}_{2} \mathrm{O}(\mathrm{wt} \%)$ content & 3 & 4 & 5 & 5 & 5 & 5 & 5 & 5 & 6 & 6 & 6 & 6 & 6 \\
\hline Set & Frit & 422 & d1-1 & 473 & P1-1 & $\mathrm{P} 2-1$ & $\mathrm{P} 2-2$ & P3-1 & $\mathrm{t} 1-1$ & 202 & 432 & $\mathrm{P} 2-3$ & $\mathrm{P} 2-4$ & P3-2 \\
\hline 1 & $\begin{array}{c}\text { Case } 8 \text { and } 8 \mathrm{a} \text { Batch } \\
1\end{array}$ & - & - & $\begin{array}{c}\text { hvisc } \\
38-42 \\
\text { TL }\end{array}$ & $\begin{array}{c}\text { hvisc } \\
26-37 \\
\text { TL }\end{array}$ & $\begin{array}{c}25-39 \\
\text { TL }\end{array}$ & $\begin{array}{c}25-39 \\
\text { TL }\end{array}$ & $\begin{array}{l}25-45 \\
\text { TL, neph }\end{array}$ & $\begin{array}{c}\text { hvisc } \\
33-39 \\
\text { TL }\end{array}$ & - & $\begin{array}{c}\text { hvisc } \\
33-43 \\
\text { Tl }\end{array}$ & $\begin{array}{c}25-42 \\
\text { TL }\end{array}$ & $\begin{array}{l}25-39 \\
\text { lvisc }\end{array}$ & $\begin{array}{c}25-44 \\
\text { TL, neph }\end{array}$ \\
\hline 1 & Case $8 \mathrm{~A}$ - Blend 1 & - & $\begin{array}{c}\text { hvisc } \\
37-41 \\
\text { TL } \\
\end{array}$ & $\begin{array}{c}\text { hvisc } \\
33-42 \\
\text { TL } \\
\end{array}$ & $\begin{array}{c}25-38 \\
\text { TL }\end{array}$ & $\begin{array}{c}25-40 \\
\text { TL }\end{array}$ & $\begin{array}{l}25-38 \\
\text { lvisc }\end{array}$ & $\begin{array}{l}25-45 \\
\text { TL }\end{array}$ & $\begin{array}{c}\text { hvisc } \\
28-40 \\
\text { TL } \\
\end{array}$ & $\begin{array}{c}\text { hvisc } \\
34-38 \\
\text { TL } \\
\end{array}$ & $\begin{array}{c}\text { hvisc } \\
29-43 \\
\text { TL } \\
\end{array}$ & $\begin{array}{c}25-42 \\
\text { TL }\end{array}$ & $\begin{array}{l}25-34 \\
\text { lvisc }\end{array}$ & $\begin{array}{l}25-44 \\
\text { TL }\end{array}$ \\
\hline 2 & Case 15 Batch 1 & - & - & - & - & - & $\begin{array}{c}25-39 \\
\text { neph }\end{array}$ & - & - & - & - & - & $\begin{array}{c}25-38 \\
\text { neph }\end{array}$ & - \\
\hline 2 & Case 15 Batch 2 & - & $\begin{array}{c}\text { hvisc } \\
40-42 \\
\text { TL }\end{array}$ & $\begin{array}{c}\text { hvisc } \\
36-43 \\
\text { TL }\end{array}$ & $\begin{array}{c}25-38 \\
\text { TL }\end{array}$ & $\begin{array}{l}25-41 \\
\text { TL }\end{array}$ & $\begin{array}{c}25-41 \\
\text { TL, lvisc }\end{array}$ & $\begin{array}{c}25-45 \\
\text { neph }\end{array}$ & $\begin{array}{c}\text { hvisc } \\
31-41 \\
\text { TL }\end{array}$ & $\begin{array}{c}\text { hvisc } \\
37-39 \\
\text { TL }\end{array}$ & $\begin{array}{c}\text { hvisc } \\
31-45 \\
\text { TL, neph }\end{array}$ & $\begin{array}{c}25-43 \\
\text { TL, neph }\end{array}$ & $\begin{array}{l}25-36 \\
\text { lvisc }\end{array}$ & $\begin{array}{c}25-44 \\
\text { neph }\end{array}$ \\
\hline 2 & Case 15 Blend 1 & - & $\begin{array}{c}\text { hvisc } \\
39-41 \\
\text { TL } \\
\end{array}$ & $\begin{array}{c}\text { hvisc } \\
35-42 \\
\text { TL } \\
\end{array}$ & $\begin{array}{c}25-37 \\
\text { TL }\end{array}$ & $\begin{array}{l}25-40 \\
\text { TL }\end{array}$ & $\begin{array}{c}25-40 \\
T L, \text { lvisc }\end{array}$ & $\begin{array}{c}25-45 \\
\text { TL }\end{array}$ & $\begin{array}{c}\text { hvisc } \\
30-40 \\
\text { TL } \\
\end{array}$ & $\begin{array}{c}\text { hvisc } \\
36-38 \\
\text { TL } \\
\end{array}$ & $\begin{array}{c}\text { hvisc } \\
31-43 \\
\text { TL }\end{array}$ & $\begin{array}{c}25-42 \\
\text { TL }\end{array}$ & $\begin{array}{l}25-36 \\
\text { lvisc }\end{array}$ & $\begin{array}{c}25-44 \\
\text { TL }\end{array}$ \\
\hline 2 & Case 15 Blend 2 & - & $\begin{array}{c}\text { hvisc } \\
39-43 \\
\text { TL } \\
\end{array}$ & $\begin{array}{c}\text { hvisc } \\
35-44 \\
\text { TL } \\
\end{array}$ & $\begin{array}{c}25-39 \\
\text { TL }\end{array}$ & $\begin{array}{l}25-41 \\
\text { TL }\end{array}$ & $\begin{array}{l}25-40 \\
\text { lvisc }\end{array}$ & $\begin{array}{c}25-45 \\
\text { neph }\end{array}$ & $\begin{array}{c}\text { hvisc } \\
30-41 \\
\text { TL } \\
\end{array}$ & $\begin{array}{c}\text { hvisc } \\
36-40 \\
\text { TL }\end{array}$ & $\begin{array}{c}\text { hvisc } \\
31-45 \\
\text { TL, neph }\end{array}$ & $\begin{array}{c}25-43 \\
\text { neph }\end{array}$ & $\begin{array}{l}25-35 \\
\text { lvisc }\end{array}$ & $\begin{array}{c}25-44 \\
\text { neph }\end{array}$ \\
\hline 3 & Case $7 b$ Batch 1 & $\begin{array}{c}\text { hvisc } \\
44-45 \\
\text { neph } \\
\end{array}$ & $\begin{array}{c}\text { hvisc } \\
40-45 \\
\text { neph } \\
\end{array}$ & $\begin{array}{c}\text { hvisc } \\
36-44 \\
\text { neph } \\
\end{array}$ & $\begin{array}{c}25-42 \\
\text { neph }\end{array}$ & $\begin{array}{c}25-42 \\
\text { neph }\end{array}$ & $\begin{array}{c}25-41 \\
\text { lvisc, neph }\end{array}$ & $\begin{array}{c}25-43 \\
\text { neph }\end{array}$ & $\begin{array}{c}\text { hvisc } \\
31-43 \\
\text { neph } \\
\end{array}$ & $\begin{array}{c}\text { hvisc } \\
37-42 \\
\text { neph } \\
\end{array}$ & $\begin{array}{c}\text { hvisc } \\
32-43 \\
\text { neph } \\
\end{array}$ & $\begin{array}{c}25-41 \\
\text { neph }\end{array}$ & $\begin{array}{l}25-37 \\
\text { lvisc }\end{array}$ & $\begin{array}{c}25-42 \\
\text { neph }\end{array}$ \\
\hline 3 & Case $7 b$ Batch 2 & - & - & - & $\begin{array}{c}\text { hvisc } \\
35-38 \\
\text { TL } \\
\end{array}$ & $\begin{array}{c}25-40 \\
\text { TL }\end{array}$ & $\begin{array}{l}25-40 \\
\text { TL }\end{array}$ & $\begin{array}{c}25-44 \\
\text { neph }\end{array}$ & - & - & $\begin{array}{c}\text { hvisc } \\
44-44 \\
\text { TL, neph }\end{array}$ & $\begin{array}{c}25-42 \\
\text { TL, neph }\end{array}$ & $\begin{array}{c}25-41 \\
\text { TL, neph }\end{array}$ & $\begin{array}{c}25-43 \\
\text { neph }\end{array}$ \\
\hline 3 & Case $7 \mathrm{~b}$ Blend 1 & $\begin{array}{c}\text { hvisc } \\
34-43 \\
\text { TL }\end{array}$ & $\begin{array}{c}\text { hvisc } \\
31-44 \\
\text { TL } \\
\end{array}$ & $\begin{array}{c}\text { hvisc } \\
28-45 \\
\text { TL } \\
\end{array}$ & $\begin{array}{c}25-40 \\
\text { TL }\end{array}$ & $\begin{array}{l}25-3 \\
\text { lvisc9 }\end{array}$ & $\begin{array}{l}25-31 \\
\text { lvisc }\end{array}$ & $\begin{array}{l}25-41 \\
\text { lvisc }\end{array}$ & $\begin{array}{c}25-43 \\
\text { TL }\end{array}$ & $\begin{array}{c}\text { hvisc } \\
28-41 \\
\text { TL }\end{array}$ & $\begin{array}{c}25-46 \\
\text { TL }\end{array}$ & $\begin{array}{l}25-38 \\
\text { lvisc }\end{array}$ & $\begin{array}{l}25-28 \\
\text { lvisc }\end{array}$ & $\begin{array}{l}25-41 \\
\text { lvisc }\end{array}$ \\
\hline 3 & Case $7 b$ Blend 2 & - & - & $\begin{array}{c}\text { hvisc } \\
41-44 \\
\text { TL }\end{array}$ & $\begin{array}{c}\text { hvisc } \\
28-39 \\
\text { TL }\end{array}$ & $\begin{array}{c}25-41 \\
\text { TL }\end{array}$ & $\begin{array}{c}25-41 \\
\text { TL }\end{array}$ & $\begin{array}{c}25-45 \\
\text { neph }\end{array}$ & $\begin{array}{c}\text { hvisc } \\
35-41 \\
\text { TL }\end{array}$ & - & $\begin{array}{c}\text { hvisc } \\
36-45 \\
\text { TL, neph }\end{array}$ & $\begin{array}{c}25-43 \\
\text { TL, neph }\end{array}$ & $\begin{array}{l}25-41 \\
\text { lvisc }\end{array}$ & $\begin{array}{c}25-44 \\
\text { neph }\end{array}$ \\
\hline
\end{tabular}


Table 5-2. Operating Windows from Nominal Assessments Expressed as WL Intervals (part 2)

Constraint not met: neph (nepheline), TL (liquidus temperature), lvisc (low viscosity - new model), hvisc (high viscosity - new model), and Del GP - durability.

\begin{tabular}{|c|c|c|c|c|c|c|c|c|c|c|c|c|c|c|}
\hline & $\mathrm{Na}_{2} \mathrm{O}(\mathrm{wt} \%)$ content & 7 & 7 & 7 & 7 & 8 & 9 & 10 & 11 & 11 & 12 & 13 & 14 & 15 \\
\hline Set & Frit & 460 & P2-5 & P3-3 & P3-4 & 418 & 426 & 425 & 200 & 417 & 320 & 431 & Y1-1 & $\mathrm{X} 1-1$ \\
\hline 1 & Case 15B - Batch 1 & - & $\begin{array}{c}25-37 \\
\text { neph }\end{array}$ & $\begin{array}{c}25-38 \\
\text { neph }\end{array}$ & $\begin{array}{c}25-39 \\
\text { neph }\end{array}$ & - & - & $\begin{array}{c}25-36 \\
\text { neph }\end{array}$ & - & $\begin{array}{c}25-35 \\
\text { neph }\end{array}$ & $\begin{array}{c}25-34 \\
\text { neph }\end{array}$ & $\begin{array}{c}25-33 \\
\text { neph }\end{array}$ & $\begin{array}{c}25-32 \\
\text { neph }\end{array}$ & $\begin{array}{c}25-31 \\
\text { neph }\end{array}$ \\
\hline 1 & Case 15B - Batch 2 & $\begin{array}{c}\text { hvisc } \\
41-42 \\
\text { TL } \\
\end{array}$ & $\begin{array}{c}25-38 \\
\text { TL }\end{array}$ & $\begin{array}{c}25-42 \\
\text { TL }\end{array}$ & $\begin{array}{c}25-43 \\
\text { TL, neph }\end{array}$ & $\begin{array}{c}\text { hvisc } \\
33-43 \\
\text { TL, neph }\end{array}$ & $\begin{array}{c}25-42 \\
\text { neph }\end{array}$ & $\begin{array}{c}25-41 \\
\text { neph }\end{array}$ & $\begin{array}{c}\text { hvisc } \\
26-38 \\
\text { neph } \\
\end{array}$ & $\begin{array}{c}25-40 \\
\text { neph }\end{array}$ & $\begin{array}{c}25-39 \\
\text { neph }\end{array}$ & $\begin{array}{c}25-37 \\
\text { neph }\end{array}$ & $\begin{array}{c}25-36 \\
\text { neph }\end{array}$ & $\begin{array}{c}25-35 \\
\text { neph }\end{array}$ \\
\hline 1 & Case $15 \mathrm{~B}$ - Blend 1 & $\begin{array}{c}25-46 \\
\text { TL }\end{array}$ & $\begin{array}{l}25-30 \\
\text { lvisc }\end{array}$ & $\begin{array}{c}25-38 \\
\text { lvisc } \\
\end{array}$ & $\begin{array}{l}25-40 \\
\text { lvisc }\end{array}$ & $\begin{array}{c}25-46 \\
\text { lvisc } \\
\end{array}$ & $\begin{array}{l}25-43 \\
\text { lvisc } \\
\end{array}$ & $\begin{array}{l}25-41 \\
\text { lvisc } \\
\end{array}$ & $\begin{array}{c}25-42 \\
\text { lvisc }\end{array}$ & $\begin{array}{l}25-38 \\
\text { lvisc } \\
\end{array}$ & $\begin{array}{l}25-35 \\
\text { lvisc } \\
\end{array}$ & - & - & - \\
\hline 1 & Case 15B - Blend 2 & $\begin{array}{c}\text { hvisc } \\
33-42 \\
\text { TL } \\
\end{array}$ & $\begin{array}{c}25-39 \\
\text { TL }\end{array}$ & $\begin{array}{c}25-43 \\
\text { TL }\end{array}$ & $\begin{array}{c}25-44 \\
\text { TL, neph }\end{array}$ & $\begin{array}{c}25-43 \\
\text { TL }\end{array}$ & $\begin{array}{c}25-43 \\
\text { neph }\end{array}$ & $\begin{array}{c}25-42 \\
\text { neph }\end{array}$ & $\begin{array}{c}25-39 \\
\text { neph }\end{array}$ & $\begin{array}{c}25-41 \\
\text { neph }\end{array}$ & $\begin{array}{c}25-39 \\
\text { neph }\end{array}$ & $\begin{array}{c}25-38 \\
\text { neph }\end{array}$ & $\begin{array}{c}25-37 \\
\text { neph }\end{array}$ & $\begin{array}{c}\text { Del GP } \\
30-36 \\
\text { neph } \\
\end{array}$ \\
\hline 1 & Case 16 - Batch 1 & - & - & - & - & - & - & - & - & - & - & - & - & - \\
\hline 1 & Case 16 - Batch 2 & - & $\begin{array}{c}25-38 \\
\text { neph }\end{array}$ & $\begin{array}{c}25-40 \\
\text { neph }\end{array}$ & $\begin{array}{c}25-40 \\
\text { neph }\end{array}$ & $\begin{array}{c}\text { hvisc } \\
40-40 \\
\text { neph } \\
\end{array}$ & $\begin{array}{c}\text { hvisc } \\
28-39 \\
\text { neph } \\
\end{array}$ & $\begin{array}{c}25-38 \\
\text { neph }\end{array}$ & $\begin{array}{c}\text { hvisc } \\
32-35 \\
\text { neph } \\
\end{array}$ & $\begin{array}{c}25-37 \\
\text { neph }\end{array}$ & $\begin{array}{c}25-35 \\
\text { neph }\end{array}$ & $\begin{array}{c}25-34 \\
\text { neph }\end{array}$ & $\begin{array}{c}25-33 \\
\text { neph }\end{array}$ & $\begin{array}{c}25-32 \\
\text { neph }\end{array}$ \\
\hline 1 & Case 16 - Blend 1 & $\begin{array}{c}25-40 \\
\text { TL }\end{array}$ & $\begin{array}{c}25-28 \\
\text { lvisc }\end{array}$ & $\begin{array}{l}25-36 \\
\text { lvisc }\end{array}$ & $\begin{array}{c}25-38 \\
\text { lvisc }\end{array}$ & $\begin{array}{c}25-41 \\
\text { TL }\end{array}$ & $\begin{array}{l}25-41 \\
\text { lvisc }\end{array}$ & $\begin{array}{c}25-39 \\
\text { lvisc }\end{array}$ & $\begin{array}{c}25-38 \\
\text { TL }\end{array}$ & $\begin{array}{c}\mathrm{R} 2 \mathrm{O} \\
30-36 \\
\text { lvisc }\end{array}$ & $\begin{array}{c}\mathrm{R} 2 \mathrm{O} \\
30-33 \\
\text { lvisc }\end{array}$ & - & - & - \\
\hline 1 & Case 16 - Blend 2 & $\begin{array}{c}\text { hvisc } \\
33-44 \\
\text { neph }\end{array}$ & $\begin{array}{c}25-41 \\
\text { neph }\end{array}$ & $\begin{array}{c}25-42 \\
\text { neph }\end{array}$ & $\begin{array}{c}25-43 \\
\text { neph }\end{array}$ & $\begin{array}{c}26-43 \\
\text { neph }\end{array}$ & $\begin{array}{c}25-41 \\
\text { neph }\end{array}$ & $\begin{array}{c}25-40 \\
\text { neph }\end{array}$ & $\begin{array}{c}25-38 \\
\text { neph }\end{array}$ & $\begin{array}{c}25-39 \\
\text { neph }\end{array}$ & $\begin{array}{c}25-38 \\
\text { neph }\end{array}$ & $\begin{array}{c}25-37 \\
\text { neph }\end{array}$ & $\begin{array}{c}25-36 \\
\text { neph }\end{array}$ & $\begin{array}{c}\text { Del GP } \\
31-34 \\
\text { neph }\end{array}$ \\
\hline 1 & Case 7 Batch 1 & $\begin{array}{c}\text { hvisc } \\
31-42 \\
\text { neph }\end{array}$ & $\begin{array}{c}25-40 \\
\text { neph }\end{array}$ & $\begin{array}{c}25-41 \\
\text { neph }\end{array}$ & $\begin{array}{c}25-42 \\
\text { neph }\end{array}$ & $\begin{array}{c}25-41 \\
\text { neph }\end{array}$ & $\begin{array}{c}25-40 \\
\text { neph }\end{array}$ & $\begin{array}{c}25-39 \\
\text { neph }\end{array}$ & $\begin{array}{c}25-37 \\
\text { neph }\end{array}$ & $\begin{array}{c}25-38 \\
\text { neph }\end{array}$ & $\begin{array}{c}25-37 \\
\text { neph }\end{array}$ & $\begin{array}{c}25-36 \\
\text { neph }\end{array}$ & $\begin{array}{c}\text { Del GP } \\
\text { 30-35 } \\
\text { neph }\end{array}$ & - \\
\hline 1 & Case 7 Batch 2 & - & $\begin{array}{c}25-38 \\
\text { TL }\end{array}$ & $\begin{array}{c}25-42 \\
\text { TL, neph }\end{array}$ & $\begin{array}{c}25-43 \\
\text { neph }\end{array}$ & $\begin{array}{c}\text { hvisc } \\
38-43 \\
\text { TL,neph }\end{array}$ & $\begin{array}{l}\text { hvisc } \\
26-42 \\
\text { neph }\end{array}$ & $\begin{array}{c}25-40 \\
\text { neph }\end{array}$ & $\begin{array}{c}\text { hvisc } \\
30-38 \\
\text { neph }\end{array}$ & $\begin{array}{c}25-39 \\
\text { neph }\end{array}$ & $\begin{array}{c}25-38 \\
\text { neph }\end{array}$ & $\begin{array}{c}25-37 \\
\text { neph }\end{array}$ & $\begin{array}{c}25-36 \\
\text { neph }\end{array}$ & $\begin{array}{c}25-34 \\
\text { neph }\end{array}$ \\
\hline 1 & Case 7 Blend 1 & $\begin{array}{c}25-46 \\
\text { TL }\end{array}$ & $\begin{array}{l}25-29 \\
\text { lvisc }\end{array}$ & $\begin{array}{l}25-37 \\
\text { lvisc }\end{array}$ & $\begin{array}{c}25-39 \\
\text { lvisc }\end{array}$ & $\begin{array}{c}25-45 \\
\text { lvisc }\end{array}$ & $\begin{array}{c}25-43 \\
\text { lvisc }\end{array}$ & $\begin{array}{c}25-40 \\
\text { lvisc }\end{array}$ & $\begin{array}{l}25-42 \\
\text { lvisc }\end{array}$ & $\begin{array}{l}25-37 \\
\text { lvisc }\end{array}$ & $\begin{array}{l}25-34 \\
\text { lvisc }\end{array}$ & - & - & - \\
\hline 1 & Case 7 Blend 2 & $\begin{array}{c}\text { hvisc } \\
35-43 \\
\text { TL } \\
\end{array}$ & $\begin{array}{c}25-39 \\
\text { TL }\end{array}$ & $\begin{array}{c}25-43 \\
\text { TL, neph }\end{array}$ & $\begin{array}{c}25-44 \\
T L, \text { neph }\end{array}$ & $\begin{array}{c}27-44 \\
\text { TL, neph }\end{array}$ & $\begin{array}{c}25-43 \\
\text { neph }\end{array}$ & $\begin{array}{c}25-41 \\
\text { neph }\end{array}$ & $\begin{array}{c}25-39 \\
\text { neph }\end{array}$ & $\begin{array}{c}25-40 \\
\text { neph }\end{array}$ & $\begin{array}{c}25-39 \\
\text { neph }\end{array}$ & $\begin{array}{c}25-38 \\
\text { neph }\end{array}$ & $\begin{array}{c}25-37 \\
\text { neph }\end{array}$ & $\begin{array}{c}28-35 \\
\text { neph }\end{array}$ \\
\hline 1 & Case 8 - Blend 1 & $\begin{array}{c}25-45 \\
\text { TL }\end{array}$ & $\begin{array}{c}25-33 \\
\text { lvisc }\end{array}$ & $\begin{array}{c}25-42 \\
\text { lvisc }\end{array}$ & $\begin{array}{c}25-44 \\
\text { lvisc }\end{array}$ & $\begin{array}{c}25-46 \\
\text { TL, neph }\end{array}$ & $\begin{array}{c}25-45 \\
\text { neph }\end{array}$ & $\begin{array}{c}25-44 \\
\text { neph }\end{array}$ & $\begin{array}{c}25-42 \\
\text { TL, neph }\end{array}$ & $\begin{array}{c}25-42 \\
\text { lvisc }\end{array}$ & $\begin{array}{c}25-39 \\
\text { lvisc }\end{array}$ & $\begin{array}{c}\text { Del GP } \\
32-35 \\
\text { lvisc }\end{array}$ & - & - \\
\hline 1 & Case 8 and $8 \mathrm{a}-$ Batch 1 & $\begin{array}{c}\text { hvisc } \\
28-44 \\
\text { TL, neph } \\
\end{array}$ & $\begin{array}{c}25-41 \\
\text { TL, neph }\end{array}$ & $\begin{array}{c}25-43 \\
\text { neph }\end{array}$ & $\begin{array}{c}25-43 \\
\text { neph }\end{array}$ & $\begin{array}{c}25-43 \\
\text { neph }\end{array}$ & $\begin{array}{c}25-42 \\
\text { neph }\end{array}$ & $\begin{array}{c}25-41 \\
\text { neph }\end{array}$ & $\begin{array}{c}25-39 \\
\text { neph }\end{array}$ & $\begin{array}{c}25-40 \\
\text { neph }\end{array}$ & $\begin{array}{c}25-39 \\
\text { neph }\end{array}$ & $\begin{array}{c}25-38 \\
\text { neph }\end{array}$ & $\begin{array}{c}33-36 \\
\text { neph }\end{array}$ & - \\
\hline
\end{tabular}


Table 5-2. Operating Windows from Nominal Assessments Expressed as WL Intervals (part 2)

Constraint not met: neph (nepheline), TL (liquidus temperature), lvisc (low viscosity - new model), hvisc (high viscosity - new model), and Del GP - durability.

\begin{tabular}{|c|c|c|c|c|c|c|c|c|c|c|c|c|c|c|}
\hline & $\mathrm{Na}_{2} \mathrm{O}(\mathrm{wt} \%)$ content & 7 & 7 & 7 & 7 & 8 & 9 & 10 & 11 & 11 & 12 & 13 & 14 & 15 \\
\hline Set & Frit & 460 & P2-5 & P3-3 & P3-4 & 418 & 426 & 425 & 200 & 417 & 320 & 431 & Y1-1 & $\mathrm{X} 1-1$ \\
\hline 1 & Case $8 \mathrm{~A}$ - Blend 1 & $\begin{array}{c}25-45 \\
\text { TL }\end{array}$ & $\begin{array}{c}25-36 \\
\text { lvisc }\end{array}$ & $\begin{array}{c}25-44 \\
\text { neph }\end{array}$ & $\begin{array}{c}25-45 \\
\text { neph }\end{array}$ & $\begin{array}{c}25-45 \\
\text { neph }\end{array}$ & $\begin{array}{c}25-44 \\
\text { neph }\end{array}$ & $\begin{array}{c}25-42 \\
\text { neph }\end{array}$ & $\begin{array}{c}25-40 \\
\text { neph }\end{array}$ & $\begin{array}{c}25-41 \\
\text { neph }\end{array}$ & $\begin{array}{c}25-40 \\
\text { neph }\end{array}$ & $\begin{array}{c}25-38 \\
\text { lvisc }\end{array}$ & 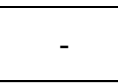 & - \\
\hline 2 & Case 15 Batch 1 & - & $\begin{array}{c}25-38 \\
\text { neph }\end{array}$ & - & - & - & - & - & - & - & $\begin{array}{l}25-33 \\
\text { hvisc }\end{array}$ & $\begin{array}{c}25-34 \\
\text { neph }\end{array}$ & $\begin{array}{c}25-33 \\
\text { neph }\end{array}$ & $\begin{array}{c}25-31 \\
\text { neph }\end{array}$ \\
\hline 2 & Case 15 Batch 2 & $\begin{array}{c}\text { hvisc } \\
26-44 \\
\text { neph }\end{array}$ & $\begin{array}{c}25-39 \\
\text { lvisc }\end{array}$ & $\begin{array}{c}25-43 \\
\text { neph }\end{array}$ & $\begin{array}{c}25-43 \\
\text { neph }\end{array}$ & $\begin{array}{c}25-43 \\
\text { neph }\end{array}$ & $\begin{array}{c}25-42 \\
\text { neph }\end{array}$ & $\begin{array}{c}25-41 \\
\text { neph }\end{array}$ & $\begin{array}{c}25-38 \\
\text { neph }\end{array}$ & $\begin{array}{c}25-39 \\
\text { neph }\end{array}$ & $\begin{array}{c}25-38 \\
\text { neph }\end{array}$ & $\begin{array}{c}25-37 \\
\text { neph }\end{array}$ & - & - \\
\hline 2 & Case 15 Blend 1 & $\begin{array}{c}\text { hvisc } \\
26-44 \\
\text { TL }\end{array}$ & $\begin{array}{c}25-39 \\
\text { lvisc }\end{array}$ & $\begin{array}{c}25-45 \\
\text { TL }\end{array}$ & $\begin{array}{c}25-46 \\
\text { TL }\end{array}$ & $\begin{array}{c}25-46 \\
\text { TL }\end{array}$ & $\begin{array}{c}25-46 \\
\text { neph }\end{array}$ & $\begin{array}{c}25-44 \\
\text { neph }\end{array}$ & $\begin{array}{c}25-42 \\
\text { TL, neph }\end{array}$ & $\begin{array}{c}25-43 \\
\text { neph }\end{array}$ & $\begin{array}{c}25-42 \\
\text { neph }\end{array}$ & $\begin{array}{l}25-41 \\
\text { lvisc, } \\
\text { neph }\end{array}$ & $\begin{array}{c}25-36 \\
\text { lvisc }\end{array}$ & - \\
\hline 2 & Case 15 Blend 2 & $\begin{array}{c}25-44 \\
\text { neph }\end{array}$ & $\begin{array}{l}25-38 \\
\text { lvisc } \\
\end{array}$ & $\begin{array}{c}25-43 \\
\text { neph }\end{array}$ & $\begin{array}{c}25-44 \\
\text { neph }\end{array}$ & $\begin{array}{c}25-43 \\
\text { neph }\end{array}$ & $\begin{array}{c}25-42 \\
\text { neph }\end{array}$ & $\begin{array}{c}25-41 \\
\text { neph }\end{array}$ & $\begin{array}{c}25-39 \\
\text { neph }\end{array}$ & $\begin{array}{c}25-40 \\
\text { neph }\end{array}$ & $\begin{array}{c}25-39 \\
\text { neph }\end{array}$ & $\begin{array}{c}25-38 \\
\text { neph }\end{array}$ & - & - \\
\hline 3 & Case 7b Batch 1 & $\begin{array}{c}\text { hvisc } \\
26-42 \\
\text { neph }\end{array}$ & $\begin{array}{c}25-39 \\
\text { neph }\end{array}$ & $\begin{array}{c}25-41 \\
\text { neph }\end{array}$ & $\begin{array}{c}25-41 \\
\text { neph }\end{array}$ & $\begin{array}{c}25-41 \\
\text { neph }\end{array}$ & $\begin{array}{c}25-40 \\
\text { neph }\end{array}$ & $\begin{array}{c}25-39 \\
\text { neph }\end{array}$ & $\begin{array}{c}25-36 \\
\text { neph }\end{array}$ & $\begin{array}{c}25-38 \\
\text { neph }\end{array}$ & $\begin{array}{c}25-36 \\
\text { neph }\end{array}$ & $\begin{array}{c}\text { Del GP } \\
31-35 \\
\text { neph } \\
\end{array}$ & - & - \\
\hline 3 & Case $7 b$ Batch 2 & $\begin{array}{c}\text { hvisc } \\
37-43 \\
\text { neph }\end{array}$ & $\begin{array}{c}25-40 \\
\text { neph }\end{array}$ & $\begin{array}{c}25-42 \\
\text { neph }\end{array}$ & $\begin{array}{c}25-42 \\
\text { neph }\end{array}$ & $\begin{array}{c}29-42 \\
\text { neph }\end{array}$ & $\begin{array}{c}25-41 \\
\text { neph }\end{array}$ & $\begin{array}{c}25-40 \\
\text { neph }\end{array}$ & $\begin{array}{c}25-37 \\
\text { neph }\end{array}$ & $\begin{array}{c}25-39 \\
\text { neph }\end{array}$ & $\begin{array}{c}25-38 \\
\text { neph }\end{array}$ & $\begin{array}{c}25-36 \\
\text { neph }\end{array}$ & $\begin{array}{c}25-35 \\
\text { neph }\end{array}$ & $\begin{array}{c}\text { Del GP } \\
29-34 \\
\text { neph } \\
\end{array}$ \\
\hline 3 & Case $7 \mathrm{~b}$ Blend 1 & $\begin{array}{c}25-47 \\
\text { TL }\end{array}$ & $\begin{array}{l}25-30 \\
\text { lvisc } \\
\end{array}$ & $\begin{array}{l}25-38 \\
\text { lvisc } \\
\end{array}$ & $\begin{array}{c}25-40 \\
\text { lvisc }\end{array}$ & $\begin{array}{c}25-46 \\
\text { lvisc } \\
\end{array}$ & $\begin{array}{c}25-44 \\
\text { lvisc }\end{array}$ & $\begin{array}{l}25-41 \\
\text { lvisc }\end{array}$ & $\begin{array}{c}25-42 \\
\text { neph }\end{array}$ & $\begin{array}{c}25-38 \\
\text { lvisc }\end{array}$ & $\begin{array}{c}25-35 \\
\text { lvisc }\end{array}$ & - & - & - \\
\hline 3 & Case $7 b$ Blend 2 & $\begin{array}{c}\text { hvisc } \\
30-44 \\
\text { neph } \\
\end{array}$ & $\begin{array}{c}25-41 \\
\text { neph }\end{array}$ & $\begin{array}{c}25-43 \\
\text { neph }\end{array}$ & $\begin{array}{c}25-43 \\
\text { neph }\end{array}$ & $\begin{array}{c}25-43 \\
\text { neph }\end{array}$ & $\begin{array}{c}25-42 \\
\text { neph }\end{array}$ & $\begin{array}{c}25-41 \\
\text { neph }\end{array}$ & $\begin{array}{c}25-38 \\
\text { neph }\end{array}$ & $\begin{array}{c}25-40 \\
\text { neph }\end{array}$ & $\begin{array}{c}25-38 \\
\text { neph }\end{array}$ & $\begin{array}{c}25-37 \\
\text { neph }\end{array}$ & $\begin{array}{c}\text { Del Gp } \\
26-36 \\
\text { neph }\end{array}$ & - \\
\hline
\end{tabular}




\subsection{Results of the Variation Stage Assessments}

As described in Section 4.3, a small set of sludge/frit glass systems was selected for evaluation by the aggressive Variation Stage assessment. How are the results from these assessments to be summarized? The answer is the interval of waste loadings for which $100 \%$ of the region's EVs meet the MAR criteria for that frit. Table 5-3 through Table 5-6 provide the summaries of the results at each waste loading from 25 to $60 \%$ for the candidate frits for each of the glass systems studied. Note that the potential for nepheline formation was considered as part of this evaluation; those entries that are shaded yellow indicate the WLs at which the nepheline constraint indicated a potential problem for some of the EVs.

For continuity and to provide a roadmap for interpretation, consider Frit 418 - Case 15 Blend 1 option (see Table 5-3). The projected operating window for the centroid composition is $25-46 \%$ with a prediction of $\mathrm{T}_{\mathrm{L}}$ limiting access to higher WLs. This is consistent with the Nominal Stage assessment as discussed in the previous section. The WL interval over which all 2464 EVs could be processed is $25-$ $37 \% \mathrm{WL}$. This is a relatively large operating window which demonstrates the robustness of Frit 418 to potential variation (even with the aggressive approach) in sludge composition. At $38 \% \mathrm{WL}, 2 \%$ (or approximately $49 \mathrm{EVs}$ ) are classified as not acceptable due to $\mathrm{T}_{\mathrm{L}}$ predictions. Potential nepheline issues do not come into play until 45\% WL (only a $3 \mathrm{WL}$ point reduction from the centroid assessment). This suggests that the initial concerns of nepheline having a dramatic negative impact on the operating windows once compositional variation was applied did not materialize. Instead, the "aggressive" nature of the approach taken resulted in significant changes in $T_{L}$ predictions to the point at which severe reductions in the operating windows occurred. More specifically, the $\pm 1 \mathrm{wt} \%$ value applied to $\mathrm{NiO}$ and $\mathrm{MnO}$ coupled with the $7.5 \%$ variation applied with $\mathrm{Fe}_{2} \mathrm{O}_{3}$ resulted in significant changes to $\mathrm{T}_{\mathrm{L}}$ predictions for the EVs. If the more "traditional" approach were to be taken in the Variation Stage assessment, the reduction in the operating window over which all the EVs could be processed would not be as severe and the window would possibly be limited based on nepheline as anticipated.

Although a detailed discussion is not provided for each system, an easy assessment to make is to assess the response of each frit to the same EVs that define a specific case. Consider Frit 425 and Frit P3-1 with the Case 15 Blend 1 option. Based on the \% of EVs considered processable, there appears to be very little, if any, difference in the projected operating windows between Frit 418 and Frit 425 . With a 2\% $\mathrm{Na}_{2} \mathrm{O}$ difference between the two frits, this is expected - given the significant impact of $\mathrm{T}_{\mathrm{L}}$ predictions. The primary difference between the two frits can be seen in the centroid assessments where the additional $2 \% \mathrm{Na}_{2} \mathrm{O}$ in Frit 425 has a negative impact on the upper WL achievable given nepheline prediction issues. The $\mathrm{Fe}_{2} \mathrm{O}_{3}$ addition to Frit P3-1 does not help the $\mathrm{T}_{\mathrm{L}}$ issue for the centroid assessment as $1 \mathrm{WL}$ point is lost as compared to the Frit 418 system. However, if one elects to suppress nepheline formation through strategic frit development efforts, Frit P3-1 does not appear to yield significant losses with respect to its ability to process the EVs. 
WSRC-RP-2006-00002

Revision 0

Table 5-3. Variation Stage MAR Results (as \% of EVs) for Case 15 Batch 1 and Blend 1.

\begin{tabular}{|c|c|c|c|c|c|c|c|c|}
\hline \multirow[b]{2}{*}{$\begin{array}{c}\% \\
\text { Waste }\end{array}$} & \multicolumn{2}{|c|}{ Case 15 Batch $1^{9}$} & \multicolumn{6}{|c|}{ Case 15 Blend 1} \\
\hline & Frit 320 & Frit 320 & Frit 418 & Frit 418 & Frit 425 & Frit 425 & Frit p3-1 & Frit $\mathrm{p} 3-1$ \\
\hline Loading & Centroid & $\begin{array}{l}\% \text { of } \\
\text { EVs }\end{array}$ & Centroid & $\begin{array}{l}\% \text { of } \\
\text { EVs }\end{array}$ & Centroid & $\begin{array}{l}\% \text { of } \\
\text { EVs }\end{array}$ & Centroid & $\begin{array}{l}\% \text { of } \\
\text { EVs }\end{array}$ \\
\hline 25 & & 73 & & 100 & & 100 & & 100 \\
\hline 26 & & 70 & & 100 & & 100 & & 100 \\
\hline 27 & & 67 & & 100 & & 100 & & 100 \\
\hline 28 & & 65 & & 100 & & 100 & & 100 \\
\hline 29 & & 63 & & 100 & & 100 & & 100 \\
\hline 30 & & 59 & & 100 & & 100 & & 100 \\
\hline 31 & & 57 & & 100 & & 100 & & 100 \\
\hline 32 & & 54 & & 100 & & 100 & & 100 \\
\hline 33 & & 53 & & 100 & & 100 & & 100 \\
\hline 34 & & 51 & & 100 & & 100 & & 100 \\
\hline 35 & newhy & 46 & & 100 & & 100 & & 100 \\
\hline 37 & newhy Neph & 8 & & 100 & & 100 & & 99.9 \\
\hline 38 & newhy Neph & & & 98 & & 98 & & 98 \\
\hline 39 & newhv Neph & & & 96 & & 96 & & 95 \\
\hline 40 & newhy Neph & & & 91 & & 91 & & 91 \\
\hline 41 & newhy Neph & & & 87 & & 87 & & 86 \\
\hline 42 & newhy Neph & & & 80 & & 80 & & 80 \\
\hline 43 & newhy Neph & & & 73 & & 64 & & 72 \\
\hline 44 & newhy Neph & & & 64 & & 46 & & 63 \\
\hline 45 & newhv Neph & & & 50 & Neph & 24 & & 52 \\
\hline 46 & newhy Neph & & & 35 & Neph & 5 & TL & 42 \\
\hline 47 & newhy Neph & & $\mathrm{TL}$ & 18 & TL Neph & 1 & TL & 25 \\
\hline 48 & newhv Neph & & TL Neph & 2 & TL Neph & & $\mathrm{TL}$ & 11 \\
\hline 49 & newhv Neph & & TL Neph & 1 & TL Neph & & $\mathrm{TL}$ & 4 \\
\hline 50 & newhv Neph & & TL Neph & & TL Neph & & TL Neph & 1 \\
\hline 51 & newhy Neph & & TL Neph & & TL Neph & & TL newlv Neph & \\
\hline 54 & newhv Neph & & TL Neph & & TL Neph & & TL newlv Neph & \\
\hline 55 & newhy Neph & & TL Neph & & TL Neph & & TL newlv Neph & \\
\hline 56 & TL newhy Neph & & TL newlv Neph & & TL newlv Neph & & TL lvisc newlv Neph & \\
\hline 57 & TL newhv Neph & & TL newlv Neph & & TL newlv Neph & & TL lvisc newlv Neph & \\
\hline 58 & TL newhv Neph & & TL newly Neph & & TL newlv Neph & & TL lvisc newlv Neph & \\
\hline 59 & TL newhv Neph & & TL newlv Neph & & TL newlv Neph & & TL lvisc newlv Neph & \\
\hline 60 & TL newhv Neph & & TL newly Neph & & TL newlv Neph & & TL lvisc newlv Neph & \\
\hline
\end{tabular}

\footnotetext{
${ }^{9}$ The shaded cells indicate that the potential for nepheline formation is predicted for some of the EVs using the 0.62 value for the constraint. Constraint not met: neph (nepheline), TL (liquidus temperature), lvisc (low viscosity - new model), hvisc (high viscosity - new model), and Del GP - durability.
} 
Table 5-4. Variation Stage MAR Results (as \% of EVs) for Case 15 Batch 2 and Blend 2.

\begin{tabular}{|c|c|c|c|c|c|c|c|c|c|c|}
\hline \multirow[b]{2}{*}{$\begin{array}{c}\% \\
\text { Waste }\end{array}$} & \multicolumn{4}{|c|}{ Case 15 Batch $2^{10}$} & \multicolumn{6}{|c|}{ Case 15 Blend 2} \\
\hline & Frit 418 & Frit 418 & Frit p3-1 & Frit $\mathrm{p} 3-1$ & Frit 418 & Frit 418 & Frit 425 & Frit 425 & Frit $\mathrm{p} 3-1$ & Frit $\mathrm{p} 3-1$ \\
\hline Loading & Centroid & $\begin{array}{c}\text { \% of } \\
\text { EVs }\end{array}$ & Centroid & $\begin{array}{c}\% \text { of } \\
\text { EVs }\end{array}$ & Centroid & $\begin{array}{c}\text { \% of } \\
\text { EVs }\end{array}$ & Centroid & $\begin{array}{c}\text { \% of } \\
\text { EVs }\end{array}$ & Centroid & $\begin{array}{c}\% \text { of } \\
\text { EVs }\end{array}$ \\
\hline 25 & & 93 & & 100 & & 100 & & 100 & & 100 \\
\hline 26 & & 100 & & 100 & & 100 & & 100 & & 100 \\
\hline 27 & & 100 & & 100 & & 100 & & 100 & & 100 \\
\hline 28 & & 100 & & 100 & & 100 & & 100 & & 100 \\
\hline 29 & & 100 & & 100 & & 100 & & 100 & & 100 \\
\hline 30 & & 100 & & 100 & & 100 & & 100 & & 100 \\
\hline 31 & & 100 & & 100 & & 100 & & 100 & & 100 \\
\hline 32 & & 100 & & 100 & & 100 & & 100 & & 100 \\
\hline 33 & & 100 & & 100 & & 100 & & 100 & & 100 \\
\hline 34 & & 100 & & 100 & & 100 & & 100 & & 100 \\
\hline 35 & & 100 & & 100 & & 100 & & 100 & & 100 \\
\hline 36 & & 100 & & 100 & & 100 & & 100 & & 100 \\
\hline 37 & & 100 & & 100 & & 100 & & 100 & & 100 \\
\hline 38 & & 99.8 & & 99.7 & & 99.9 & & 100 & & 99.9 \\
\hline 39 & & 98 & & 96 & & 98 & & 100 & & 98 \\
\hline 40 & & 93 & & 93 & & 94 & & 91 & & 94 \\
\hline 41 & & 89 & & 88 & & 90 & & 76 & & 90 \\
\hline 42 & & 69 & & 83 & & 77 & Neph & 31 & & 85 \\
\hline 43 & & 50 & & 71 & & 61 & Neph & 11 & & 81 \\
\hline 44 & Neph & 9 & & 54 & Neph & 31 & Neph & 5 & & 61 \\
\hline 45 & Neph & 3 & & 29 & Neph & 6 & Neph & & & 41 \\
\hline 46 & Neph & & Neph & 4 & Neph & 2 & Neph & & Neph & 15 \\
\hline 47 & Neph & & TL Neph & 1 & Neph & & Neph & & Neph & 3 \\
\hline 48 & TL Neph & & TL Neph & & TL Neph & & Neph & & TL Neph & 1 \\
\hline 49 & TL Neph & & TL Neph & & TL Neph & & Neph & & TL Neph & \\
\hline 50 & TL Neph & & TL Neph & & TL Neph & & TL Neph & & TL Neph & \\
\hline 51 & TL Neph & & TL Neph & & TL Neph & & TL newlv Neph & & TL newlv Neph & \\
\hline 52 & TL Neph & & TL newlv Neph & & TL Neph & & TL newlv Neph & & TL newlv Neph & \\
\hline 53 & TL Neph & & TL newlv Neph & & TL Neph & & TL newlv Neph & & TL newlv Neph & \\
\hline 54 & TL Neph & & TL newlv Neph & & TL Neph & & TL newlv Neph & & TL newlv Neph & \\
\hline 55 & TL Neph & & TL newlv Neph & & TL Neph & & TL newlv Neph & & TL newlv Neph & \\
\hline 56 & TL Neph & & TL newlv Neph & & TL newlv Neph & & TL newlv Neph & & TL newlv Neph & \\
\hline 57 & TL newlv Neph & & TL newlv Neph & & TL newlv Neph & & TL newlv Neph & & TL newlv Neph & \\
\hline 58 & TL newlv Neph & & TL newlv Neph & & TL newlv Neph & & TL newlv Neph & & TL newlv Neph & \\
\hline 59 & TL newlv Neph & & TL newlv Neph & & TL newlv Neph & & TL newlv Neph & & TL newlv Neph & \\
\hline 60 & TL newlv Neph & & TL newlv Neph & & TL newlv Neph & & TL newlv Neph & & TL newlv Neph & \\
\hline
\end{tabular}

\footnotetext{
${ }^{10}$ The shaded cells indicate that the potential for nepheline formation is predicted for some of the EVs using the 0.62 value for the constraint. Constraint not met: neph (nepheline), TL (liquidus
} temperature), lvisc (low viscosity - new model), hvisc (high viscosity - new model), and Del GP - durability. 
Table 5-5. Variation Stage MAR Results (as \% of EVs) for Case 7b Batch 1 and Blend 1.

\begin{tabular}{|c|c|c|c|c|c|c|c|c|c|c|}
\hline & \multicolumn{4}{|c|}{ Case $7 \mathrm{~b}$ Batch $1^{11}$} & \multicolumn{6}{|c|}{ Case $7 \mathrm{~b}$ Blend 1} \\
\hline$\%$ Waste & Frit 418 & Frit 418 & Frit p3-1 & Frit p3-1 & Frit 418 & Frit 418 & Frit 425 & Frit 425 & Frit p3-1 & Frit p3-1 \\
\hline Loading & Centroid & $\%$ of EVs & Centroid & $\%$ of EVs & Centroid & $\%$ of EVs & Centroid & $\%$ of EVs & Centroid & $\%$ of EVs \\
\hline 25 & & 89 & & 100 & & 100 & & 100 & & 100 \\
\hline 26 & & 95 & & 100 & & 100 & & 100 & & 100 \\
\hline 27 & & 100 & & 100 & & 100 & & 100 & & 100 \\
\hline 28 & & 100 & & 100 & & 100 & & 100 & & 100 \\
\hline 29 & & 100 & & 100 & & 100 & & 100 & & 100 \\
\hline 30 & & 100 & & 100 & & 100 & & 100 & & 100 \\
\hline 31 & & 100 & & 100 & & 100 & & 100 & & 100 \\
\hline 32 & & 100 & & 100 & & 100 & & 100 & & 100 \\
\hline 33 & & 100 & & 100 & & 100 & & 100 & & 100 \\
\hline 34 & & 100 & & 100 & & 100 & & 100 & & 100 \\
\hline 35 & & 100 & & 100 & & 100 & & 100 & & 100 \\
\hline 36 & & 100 & & 100 & & 100 & & 100 & & 100 \\
\hline 37 & & 100 & & 100 & & 100 & & 100 & & 100 \\
\hline 38 & & 100 & & 100 & & 100 & & 91 & & 91 \\
\hline 39 & & 100 & & 100 & & 99.8 & & 82 & & 82 \\
\hline 40 & & 87 & & 100 & & 98 & & 75 & & 73 \\
\hline 41 & & 65 & & 100 & & 95 & & 59 & & 55 \\
\hline 42 & Neph & 18 & & 85 & & 89 & newlv & 47 & newlv & 41 \\
\hline 43 & Neph & 9 & & 53 & & 77 & newlv & 31 & newlv & 25 \\
\hline 44 & Neph & & Neph & 16 & & 64 & newlv & 19 & newlv & 16 \\
\hline 45 & Neph & & Neph & 7 & & 50 & newlv & 12 & newlv & 10 \\
\hline 46 & Neph & & Neph & & & 30 & newlv Neph & 1 & newlv & 4 \\
\hline 47 & Neph & & Neph & & newlv & 16 & newlv Neph & & newlv & 2 \\
\hline 48 & Neph & & Neph & & newlv Neph & 6 & newlv Neph & & newlv & \\
\hline 49 & Neph & & Neph & & TL newlv Neph & & newlv Neph & & TL newlv & \\
\hline 50 & Neph & & Neph & & TL newlv Neph & & newlv Neph & & TL newlv Neph & \\
\hline 51 & Neph & & Neph & & TL newlv Neph & & TL newlv Neph & & TL newlv Neph & \\
\hline 52 & Neph & & Neph & & TL newlv Neph & & TL newlv Neph & & TL newlv Neph & \\
\hline 53 & Neph & & newlv Neph & & TL newlv Neph & & TL newlv Neph & & TL newlv Neph & \\
\hline 54 & Neph & & newlv Neph & & TL newlv Neph & & TL newlv Neph & & TL newlv Neph & \\
\hline 55 & Neph & & TL newlv Neph & & TL newlv Neph & & TL newlv Neph & & TL newlv Neph & \\
\hline 56 & TL Neph & & TL newlv Neph & & TL newlv Neph & & TL newlv Neph & & TL newlv Neph & \\
\hline 57 & TL Neph & & TL newlv Neph & & TL newlv Neph & & TL newlv Neph & & TL newlv Neph & \\
\hline 58 & TL newlv Neph & & TL newlv Neph & & TL newlv Neph & & TL newlv Neph & & TL newlv Neph & \\
\hline 59 & TL newlv Neph & & TL newlv Neph & & TL newlv Neph & & TL newlv Neph & & TL newlv Neph & \\
\hline 60 & TL newlv Neph & & TL newlv Neph & & TL newlv Neph & & TL newlv Neph & & TL newlv Neph & \\
\hline
\end{tabular}

\footnotetext{
${ }^{11}$ The shaded cells indicate that the potential for nepheline formation is predicted for some of the EVs using the 0.62 value for the constraint. Constraint not met: neph (nepheline), TL (liquidus temperature), lvisc (low viscosity - new model), hvisc (high viscosity - new model), and Del GP - durability
} 
Table 5-6. Variation Stage MAR Results (as \% of EVs) for Case 7b Batch 2 and Blend 2.

\begin{tabular}{|c|c|c|c|c|c|c|c|c|c|c|}
\hline & \multicolumn{4}{|c|}{ Case $7 \mathrm{~b}$ Batch $2^{12}$} & \multicolumn{6}{|c|}{ Case $7 \mathrm{~b}$ Blend 2} \\
\hline$\%$ Waste & Frit 418 & Frit 418 & Frit $\mathrm{p} 3-1$ & Frit p3-1 & Frit 418 & Frit 418 & Frit 425 & Frit 425 & Frit p3-1 & Frit p3-1 \\
\hline Loading & Centroid & $\%$ of EVs & Centroid & $\%$ of EVs & Centroid & $\%$ of EVs & Centroid & $\%$ of EVs & Centroid & $\%$ of EVs \\
\hline 25 & newhv & 31 & & 100 & & 67 & & 100 & & 100 \\
\hline 26 & newhy & 34 & & 100 & & 70 & & 100 & & 100 \\
\hline 27 & newhv & 39 & & 100 & & 77 & & 100 & & 100 \\
\hline 28 & newhv & 42 & & 100 & & 80 & & 100 & & 100 \\
\hline 29 & & 50 & & 100 & & 88 & & 100 & & 100 \\
\hline 30 & & 58 & & 100 & & 91 & & 100 & & 100 \\
\hline 31 & & 60 & & 100 & & 93 & & 100 & & 100 \\
\hline 32 & & 65 & & 100 & & 97 & & 100 & & 100 \\
\hline 33 & & 66 & & 100 & & 100 & & 100 & & 100 \\
\hline 34 & & 68 & & 100 & & 100 & & 100 & & 100 \\
\hline 35 & & 70 & & 100 & & 100 & & 100 & & 100 \\
\hline 36 & & 73 & & 100 & & 100 & & 100 & & 100 \\
\hline 37 & & 78 & & 100 & & 100 & & 100 & & 100 \\
\hline 38 & & 79 & & 99 & & 99.9 & & 100 & & 99.9 \\
\hline 39 & & 79 & & 95 & & 98 & & 100 & & 98 \\
\hline 40 & & 77 & & 91 & & 94 & & 85 & & 93 \\
\hline 41 & & 65 & & 86 & & 90 & & 63 & & 90 \\
\hline 42 & & 45 & & 81 & & 72 & Neph & 18 & & 84 \\
\hline 43 & Neph & 22 & & 60 & & 51 & Neph & 7 & & 80 \\
\hline 44 & Neph & 5 & & 40 & Neph & 10 & Neph & & & 57 \\
\hline 45 & Neph & & Neph & 14 & Neph & 5 & Neph & & & 40 \\
\hline 46 & Neph & & Neph & 1 & Neph & & Neph & & Neph & 6 \\
\hline 47 & TL Neph & & TL Neph & & Neph & & Neph & & Neph & 2 \\
\hline 48 & TL Neph & & TL Neph & & TL Neph & & Neph & & TL Neph & \\
\hline 49 & TL Neph & & TL Neph & & TL Neph & & Neph & & TL Neph & \\
\hline 50 & TL Neph & & TL Neph & & TL Neph & & TL Neph & & TL Neph & \\
\hline 51 & TL Neph & & TL Neph & & TL Neph & & TL Neph & & TL Neph & \\
\hline 52 & TL Neph & & TL Neph & & TL Neph & & TL Neph & & TL Neph & \\
\hline 53 & TL Neph & & TL Neph & & TL Neph & & TL Neph & & TL Neph & \\
\hline 54 & TL Neph & & TL Neph & & TL Neph & & TL Neph & & TL Neph & \\
\hline 55 & TL Neph & & TL Neph & & TL Neph & & TL Neph & & TL Neph & \\
\hline 56 & TL Neph & & TL Neph & & TL Neph & & TL Neph & & TL Neph & \\
\hline 57 & TL Neph & & TL Neph & & TL Neph & & TL newlv Neph & & TL newlv Neph & \\
\hline 58 & TL Neph & & TL Neph & & TL Neph & & TL newlv Neph & & TL newlv Neph & \\
\hline 59 & TL Neph & & TL Neph & & TL Neph & & TL newlv Neph & & TL newlv Neph & \\
\hline 60 & TL Neph & & TL Neph & & TL Neph & & TL newlv Neph & & TL newlv Neph & \\
\hline
\end{tabular}

\footnotetext{
${ }^{12}$ The shaded cells indicate that the potential for nepheline formation is predicted for some of the EVs using the 0.62 value for the constraint. Constraint not met: neph (nepheline), TL (liquidus temperature), lvisc (low viscosity - new model), hvisc (high viscosity - new model), and Del GP - durability.
} 
WSRC-RP-2006-00002

Revision 0

Table 5-7 provides a summary of these results for the EVs by showing the first constraint(s), using the same terms as used in Tables 5-3 through 5-6, that limit(s) the operating window at either end of the 25 to $60 \%$ interval of WLs.

Table 5-7. Intervals with $100 \%$ EVs Acceptable and Limiting Constraint(s)

\begin{tabular}{|c|c|c|c|c|c|}
\hline Case & Sludge Option & Frit 320 & Frit 418 & Frit 425 & Frit P3-1 \\
\hline 15 & Batch 1 & - & N/A & $\mathrm{N} / \mathrm{A}$ & $\mathrm{N} / \mathrm{A}$ \\
\hline 15 & Batch 2 & N/A & $\begin{array}{c}\text { hvisc } \\
26-37 \\
\text { TL }\end{array}$ & N/A & $\begin{array}{c}25-37 \\
\text { TL }\end{array}$ \\
\hline 15 & Blend 1 & N/A & $\begin{array}{c}25-37 \\
\text { TL }\end{array}$ & $\begin{array}{c}25-37 \\
\text { TL }\end{array}$ & $\begin{array}{c}25-36 \\
\text { TL }\end{array}$ \\
\hline 15 & Blend 2 & - & $\begin{array}{c}25-37 \\
\text { TL }\end{array}$ & $\begin{array}{c}25-39 \\
\text { Neph, TL }\end{array}$ & $\begin{array}{c}25-37 \\
\text { TL }\end{array}$ \\
\hline $7 \mathrm{~B}$ & Batch 1 & N/A & $\begin{array}{c}\text { hvisc } \\
27-39 \\
\text { neph }\end{array}$ & N/A & $\begin{array}{c}25-41 \\
\text { neph }\end{array}$ \\
\hline $7 \mathrm{~B}$ & Batch 2 & N/A & - & - & $\begin{array}{c}25-37 \\
\text { TL }\end{array}$ \\
\hline $7 \mathrm{~B}$ & Blend 1 & N/A & $\begin{array}{c}25-38 \\
\text { TL }\end{array}$ & $\begin{array}{c}25-37 \\
\text { lvisc }\end{array}$ & $\begin{array}{c}25-37 \\
\text { lvisc }\end{array}$ \\
\hline $7 \mathrm{~B}$ & Blend 2 & N/A & $\begin{array}{c}\text { hvisc } \\
33-37 \\
\text { TL }\end{array}$ & $\begin{array}{c}25-39 \\
\text { TL, neph }\end{array}$ & $\begin{array}{c}25-37 \\
\text { TL }\end{array}$ \\
\hline
\end{tabular}

\subsection{SUMMARY}

The objective of this report is to document technical information that has been provided to CBU personnel as part of the frit development support for SB4. The information presented in this report includes the resulting projected operating windows (expressed in terms of waste loading) for various sludge blending and/or washing options when coupled with candidate frits of interest. Although the Nominal Stage assessment serves as the primary tool for these evaluations, select systems were also assessed using the Variation Stage in which compositional uncertainties were added. In addition, assessments of the impacts of nepheline formation potential and the $\mathrm{SO}_{4}{ }^{=}$solubility limit on the projected operating windows are also provided. Although this information was used as part of the technical basis from which the preferred SB4 preparation plan was selected, none of the options presented in this report is the preferred plan. Therefore, the information is presented without significant interpretation but the details are provided so additional insight can be made if desired. Details regarding the evaluation of the preferred preparation plan are documented in Peeler and Edwards (2006).

Although a detailed discussion is not provided, below are some high level observations from the datasets:

(1) A comparison of the Nominal Stage assessments with the centroids from the Variation Stage assessments shows consistency between the two methods.

(2) The impact of nepheline formation does not appear to be as significant (in terms of access to upper WLs) as anticipated once compositional variations are applied. In fact, the reduction in upper WLs between the nominal (or centroid) assessment and that for the EV-based evaluation was typically $1-3$ percentage points. The more severe impact resulted from predictions associated with $\mathrm{T}_{\mathrm{L}}$.

(3) The "aggressive" approach taken in the Variation Stage assessment appears to severely impact the WL interval over which all of the EVs could be processed. Applying the $\pm 1 \mathrm{wt} \%$ value to $\mathrm{NiO}$ and $\mathrm{MnO}$ coupled with the $7.5 \%$ variation applied to $\mathrm{Fe}_{2} \mathrm{O}_{3}$ resulted in significant changes in 
the $\mathrm{T}_{\mathrm{L}}$ predictions for select EVs. If the more "traditional" approach were to be taken, the reduction in the operating windows over which all the EVs could be processed would not be as severe and possibly could be defined based on nepheline as anticipated.

(4) It appears that the "P-series" of frits (i.e., the $\mathrm{Fe}_{2} \mathrm{O}_{3}$ based) have the possibility to mitigate nepheline formation allowing access to higher WLs for select sludge systems. If true, then one may elect to utilize a $\mathrm{Fe}_{2} \mathrm{O}_{3}$-based frit to process one of the high $\mathrm{Al}_{2} \mathrm{O}_{3}$-based sludges. This latter statement assumes that there is no negative impact on melt rate and/or waste throughput - critical processing factors that were not addressed in this report.

(5) With only one exception (i.e., Case 16), projected operating windows for all of the flowsheets considered could be identified using strategic frit development strategies. It is noted that some of the operating windows are relatively small and have maximum WLs that may not meet expectations - but windows do exist.

Frit selection will ultimately be dependent on the strategy (or risks) one elects to take for SB4. More specifically, a conservative (and potentially bounding) strategy would be to avoid nepheline formation altogether through strategic frit development efforts or at least suppress it to WLs of $50 \%$ of higher. At these higher WLs, the probability of another property limiting acceptability is extremely high - thus taking nepheline and its potential negative impact out of the SB4 picture. To do this, one may need to reduce the $\mathrm{Na}_{2} \mathrm{O}$ content and/or increase the $\mathrm{SiO}_{2}$ content of the frit; both of which may lead to relatively slow melt rates. The other extreme would be to push the $\mathrm{Na}_{2} \mathrm{O}$ concentrations in glass to the point at which WL and/or melt rate may be optimized, but this strategy could put DWPF on the edge of compromising product quality - a condition to be avoided. The answer or most appropriate strategy may lie somewhere between these two extremes. That is, one should attempt to balance the probability (or possibility) to mitigate nepheline formation while gaining as much advantage in melt rate and/or WL space as possible. To perform such a balancing act, more information than presented in this report is required (e.g., melt rate information will be critical). The concept of frit selection to meet processing goals is explored more thoroughly in Peeler and Edwards (2006).

\subsection{REFERENCES}

\section{ASTM 2002. Standard Test Methods for Determining Chemical Durability of Nuclear Waste} Glasses: The Product Consistency Test (PCT), ASTM C-1285-2002.

Brown KG, CM Jantzen, and G Ritzhaupt. 2001. Relating Liquidus Temperature to Composition for Defense Waste Processing Facility (DWPF) Process Control, WSRC-TR-2001-00520, Revision 0, Westinghouse Savannah River Company, Aiken, South Carolina.

Brown, KG, RL Postles, and TB Edwards, 2002. SME Acceptability Determination for DWPF

Process Control, WSRC-TR-95-0364, Revision 4, Westinghouse Savannah River Company, Aiken, South Carolina.

Edwards, TB, DK Peeler, and SL Marra. 2003. Revisiting the Prediction Limits for Acceptable Durability, WSRC-TR-2003-00510, Revision 0, Westinghouse Savannah River Company, Aiken, South Carolina.

Jantzen, CM, JB Pickett, KG Brown, TB Edwards, and DC Beam. 1995. Process/Product Models for the Defense Waste Processing Facility (DWPF): Part I. Predicting Glass Durability from Composition Using a Thermodynamic Hydration Energy Reaction Model (THERMO) (U), WSRCTR-93-672, Revision 1, Volume 1, Westinghouse Savannah River Company, Aiken, South Carolina. 
Jantzen, CM. 2005. The Impacts of Uranium and Thorium on the Defense Waste Processing Facility (DWPF) Viscosity Model, WSRC-TR-2004-00311, Revision 0, Westinghouse Savannah River Company, Aiken, South Carolina.

Li, H., P. Hrma, J.D. Vienna, M. Qian, Y. Su, and D.E. Smith. 2003. Effects of $\mathbf{A l}_{2} \mathbf{O}_{3}, \mathbf{B}_{2} \mathbf{O}_{3}, \mathbf{N a}_{2} \mathbf{O}$, and $\mathrm{SiO}_{2}$ on Nepheline Formation in Borosilicate Glasses: Chemical and Physical Correlations, Journal of Non-Crystalline Solids, 331, pgs. $202-216$.

Lilliston, GR. 2005. Development of Elemental Sludge Compositions for Variations of Sludge Batch 4 (SB4), CBU-PIT-2004-00011, Revision 1, Westinghouse Savannah River Company, Aiken, South Carolina.

Lorier, TH, IA Reamer, and RJ Workman. 2005. Initial Sulfate Solubility Study for Sludge Batch 4 (SB4), WSRC-TR-2005-00213, Revision 0, Westinghouse Savannah River Company, Aiken, South Carolina.

Peeler, DK. 2004. Task Technical \& QA Plan: Sludge Batch and MCU Frit Optimization, WSRCRP-2004-00746, Revision 0, Westinghouse Savannah River Company, Aiken, South Carolina.

Peeler, DK and TB Edwards. 2002. Frit Development for Sludge Batch 3, WSRC-TR-2002-00491, Revision 0, Westinghouse Savannah River Company, Aiken, South Carolina.

Peeler, DK and TB Edwards. 2005. Frit Development Efforts for Sludge Batch 4 (SB4): ModelBased Assessments, WSRC-TR-2005-00103, Revision 0, Westinghouse Savannah River Company, Aiken, South Carolina.

Peeler, DK and TB Edwards. 2006. Model Based Assessments for the Baseline Sludge Batch 4 (Case 15C) Preparation Plan, WSRC-TR-2006-000, Revision 0, Westinghouse Savannah River Company, Aiken, South Carolina.

Peeler, DK, CC Herman, and TB Edwards. 2005a. The Impact of MCU on the Sludge Batch 4 (SB4) Variability Study,WSRC-TR-2005-00041, Revision 0, Westinghouse Savannah River Company, Aiken, South Carolina.

Peeler, DK, TB Edwards, IA Reamer, and RJ Workman. 2005b. Nepheline Formation Study for Sludge Batch 4 (SB4): Phase 1 Experimental Results, WSRC-TR-2005-00371, Revision 0, Westinghouse Savannah River Company, Aiken, South Carolina.

Peeler, DK, TB Edwards, IA Reamer, and RJ Workman. 2006. Nepheline Formation Study for Sludge Batch 4 (SB4): Phase 2 Experimental Results, WSRC-TR-2006-00006, Revision 0, Westinghouse Savannah River Company, Aiken, South Carolina.

SAS Institute, Inc. 2002. JMP® Statistics and Graphics Guide: JMP Version 5.1.2, SAS Institute, Inc, Cary, North Carolina.

Shah, HB. 2006. Estimate of Sludge Batch 4 Calcine Composition Additional Cases for Final Recommendation, CBU-PIT-2006-00011, Revision 0, Westinghouse Savannah River Company, Aiken, South Carolina.

Washburn, FA. 2004. Technical Task Request: Sludge Batch 4 and MCU Frit Optimization, HLW/DWPF/TTR-2004-0025, Revision 0, Westinghouse Savannah River Company, Aiken, South Carolina. 
WSRC-RP-2006-00002

Revision 0

\section{Appendix A}


Exhibit A1. Results for MAR Assessments of the Nominal Stage (part 1)

Constraint not met: neph (nepheline), TL (liquidus temperature), newlv (low viscosity - new model), newhv (high viscosity - new model), and Del GP - durability

\begin{tabular}{|c|c|c|c|c|c|c|c|c|c|}
\hline & & $\mathrm{Na} 2 \mathrm{O}$ & 0.03 & 0.04 & 0.05 & 0.05 & 0.05 & 0.05 & 0.05 \\
\hline Set & Type & Sludge Loading (\%) & 422 & $\mathrm{~d} 1-1$ & 473 & P1-1 & P2-1 & P2-2 & P3-1 \\
\hline 1 & Case 15B - Batch 1 & 25 & newhv & newhy & newhy & newhv & & & \\
\hline 1 & Case 15B - Batch 1 & 26 & newhv & newhv & newhv & newhv & & & \\
\hline 1 & Case 15B - Batch 1 & 27 & newhv & newhv & newhy & newhy & & & \\
\hline 1 & Case 15B - Batch 1 & 28 & newhy & newhy & newhy & newhy & & & \\
\hline 1 & Case 15B - Batch 1 & 29 & newhv & newhv & newhv & newhy & & & \\
\hline 1 & Case 15B - Batch 1 & 30 & newhy & newhy & newhv & newhy & & & \\
\hline 1 & Case 15B - Batch 1 & 31 & newhv & newhv & newhy & newhy & & & \\
\hline 1 & Case 15B - Batch 1 & 32 & newhv & newhy & newhy & newhy & & & \\
\hline 1 & Case 15B - Batch 1 & 33 & newhv & newhv & newhv & newhv & & & \\
\hline 1 & Case 15B - Batch 1 & 34 & newhy & newhv & newhv & newhy & & & \\
\hline 1 & Case 15B - Batch 1 & 35 & newhy & newhv & newhy & newhy & & & \\
\hline 1 & Case 15B - Batch 1 & 36 & newhv & newhy & newhy & newhv & & & \\
\hline 1 & Case 15B - Batch 1 & 37 & newhv & newhv & newhv & newhv & & & \\
\hline 1 & Case 15B - Batch 1 & 38 & newhv & newhv & newhv & newhv & & & \\
\hline 1 & Case 15B - Batch 1 & 39 & newhy & newhv & newhy & newhv & & Neph & \\
\hline 1 & Case 15B - Batch 1 & 40 & newhv & newhv & newhv & newhv & Neph & Neph & \\
\hline 1 & Case 15B - Batch 1 & 41 & newhy & newhy & newhy & newhv Neph & Neph & Neph & Neph \\
\hline 1 & Case 15B - Batch 1 & 42 & newhv & newhv & newhy Neph & newhy Neph & Neph & Neph & Neph \\
\hline 1 & Case 15B - Batch 1 & 43 & newhv & newhv Neph & newhv Neph & newhv Neph & Neph & Neph & Neph \\
\hline 1 & Case 15B - Batch 1 & 44 & newhv Neph & newhv Neph & newhv Neph & newhv Neph & Neph & Neph & Neph \\
\hline 1 & Case 15B - Batch 1 & 45 & newhv Neph & newhv Neph & newhy Neph & newhy Neph & Neph & Neph & Neph \\
\hline 1 & Case 15B - Batch 1 & 46 & newhv Neph & newhy Neph & newhv Neph & newhv Neph & Neph & Neph & Neph \\
\hline 1 & Case 15B - Batch 1 & 47 & newhv Neph & newhv Neph & newhv Neph & newhv Neph & Neph & Neph & Neph \\
\hline 1 & Case 15B - Batch 1 & 48 & newhv Neph & newhy Neph & newhv Neph & newhv Neph & Neph & Neph & Neph \\
\hline 1 & Case 15B - Batch 1 & 49 & newhv Neph & newhv Neph & newhv Neph & newhy Neph & Neph & Neph & Neph \\
\hline 1 & Case 15B - Batch 1 & 50 & newhv Neph & newhv Neph & newhv Neph & newhv Neph & Neph & Neph & Neph \\
\hline 1 & Case 15B - Batch 1 & 51 & newhv Neph & newhv Neph & newhv Neph & newhv Neph & Neph & Neph & Neph \\
\hline 1 & Case 15B - Batch 1 & 52 & newhv Neph & newhv Neph & newhv Neph & newhv Neph & Neph & Neph & Neph \\
\hline 1 & Case 15B - Batch 1 & 53 & newhv Neph & newhy Neph & newhv Neph & newhv Neph & Neph & Neph & Neph \\
\hline 1 & Case 15B - Batch 1 & 54 & newhv Neph & newhv Neph & newhv Neph & TL newhy Neph & Neph & Neph & Neph \\
\hline 1 & Case 15B - Batch 1 & 55 & newhv Neph & newhv Neph & newhv Neph & TL newhv Neph & Neph & TL Neph & Neph \\
\hline 1 & Case 15B - Batch 1 & 56 & newhv Neph & newhy Neph & newhv Neph & TL newhy Neph & TL Neph & TL Neph & Neph \\
\hline 1 & Case 15B - Batch 1 & 57 & newhv Neph & newhv Neph & newhv Neph & TL newhy Neph & TL Neph & TL Neph & Neph \\
\hline 1 & Case 15B - Batch 1 & 58 & TL newhy Neph & newhv Neph & newhv Neph & TL newhy Neph & TL Neph & TL Neph & Neph \\
\hline 1 & Case 15B - Batch 1 & 59 & TL newhy Neph & TL newhy Neph & newhv Neph & TL newhy Neph & TL Neph & TL Neph & Neph \\
\hline 1 & Case 15B - Batch 1 & 60 & TL newhy Neph & TL newhy Neph & TL newhy Neph & TL newhy Neph & TL Neph & TL Neph & Neph \\
\hline 1 & Case 15B - Batch 2 & 25 & newhy & newhv & newhv & newhv & & & \\
\hline 1 & Case 15B - Batch 2 & 26 & newhv & newhv & newhy & newhy & & & \\
\hline 1 & Case 15B - Batch 2 & 27 & newhv & newhv & newhv & newhv & & & \\
\hline 1 & Case 15B - Batch 2 & 28 & newhy & newhy & newhy & newhy & & & \\
\hline 1 & Case 15B - Batch 2 & 29 & newhv & newhy & newhv & newhv & & & \\
\hline 1 & Case 15B - Batch 2 & 30 & newhv & newhv & newhy & newhv & & & \\
\hline 1 & Case 15B - Batch 2 & 31 & newhy & newhv & newhy & newhy & & & \\
\hline 1 & Case 15B - Batch 2 & 32 & newhv & newhy & newhv & newhv & & & \\
\hline 1 & Case 15B - Batch 2 & 33 & newhv & newhy & newhv & newhv & & & \\
\hline 1 & Case 15B - Batch 2 & 34 & newhv & newhv & newhy & newhv & & & \\
\hline 1 & Case 15B - Batch 2 & 35 & newhv & newhv & newhy & TL newhy & & & \\
\hline 1 & Case 15B - Batch 2 & 36 & newhv & newhv & newhv & TL newhy & & & \\
\hline 1 & Case 15B - Batch 2 & 37 & newhv & newhv & newhv & TL newhv & & & \\
\hline 1 & Case 15B - Batch 2 & 38 & TL newhy & newhv & newhv & TL newhy & $\mathrm{TL}$ & $\mathrm{TL}$ & \\
\hline 1 & Case 15B - Batch 2 & 39 & TL newhv & TL newhv & newhy & $\mathrm{TL}$ & $\mathrm{TL}$ & $\mathrm{TL}$ & \\
\hline 1 & Case 15B - Batch 2 & 40 & TL newhv & TL newhv & TL newhv & $\mathrm{TL}$ & $\mathrm{TL}$ & $\mathrm{TL}$ & \\
\hline
\end{tabular}


Exhibit A1. Results for MAR Assessments of the Nominal Stage (part 1)

Constraint not met: neph (nepheline), TL (liquidus temperature), newlv (low viscosity - new model), newhv (high viscosity - new model), and Del GP - durability

\begin{tabular}{|c|c|c|c|c|c|c|c|c|c|}
\hline & & $\begin{array}{l}\mathrm{Na} 2 \mathrm{O} \\
\end{array}$ & 0.03 & 0.04 & 0.05 & 0.05 & 0.05 & 0.05 & 0.05 \\
\hline Set & $\begin{array}{c}\text { Type } \\
\end{array}$ & Sludge Loading (\%) & 422 & $\mathrm{~d} 1-1$ & 473 & P1-1 & P2-1 & P2-2 & P3-1 \\
\hline 1 & Case 15B - Batch 2 & 41 & TL newhy & TL newhy & TL newhv & $\mathrm{TL}$ & $\mathrm{TL}$ & $\mathrm{TL}$ & \\
\hline 1 & Case 15B - Batch 2 & 42 & TL newhv & TL newhv & TL newhv & $\mathrm{TL}$ & $\mathrm{TL}$ & $\mathrm{TL}$ & \\
\hline 1 & Case 15B - Batch 2 & 43 & TL newhy & TL newhy & TL newhy & $\mathrm{TL}$ & $\mathrm{TL}$ & $\mathrm{TL}$ & $\mathrm{TL}$ \\
\hline 1 & Case 15B - Batch 2 & 44 & TL newhy & TL newhy & TL newhy & TL & $\mathrm{TL}$ & TL Neph & $\mathrm{TL}$ \\
\hline 1 & $\begin{array}{l}\text { Case 15B - Batch } 2 \\
\end{array}$ & 45 & TL newhy & TL newhy & TL newhy & TL Neph & TL Neph & TL Neph & $\mathrm{TL}$ \\
\hline 1 & Case 15B - Batch 2 & 46 & TL newhy & TL newhv & TL newhv & TL Neph & TL Neph & TL Neph & TL Neph \\
\hline 1 & Case 15B - Batch 2 & 47 & TL newhy & TL newhy & TL newhv Neph & TL Neph & TL Neph & TL Neph & TL Neph \\
\hline 1 & Case 15B - Batch 2 & 48 & TL newhy & TL newhy Neph & TL newhy Neph & TL Neph & TL Neph & TL Neph & TL Neph \\
\hline 1 & Case 15B - Batch 2 & 49 & TL newhv Neph & TL newhv Neph & TL newhy Neph & TL Neph & TL Neph & TL Neph & TL Neph \\
\hline 1 & Case 15B - Batch 2 & 50 & TL newhy Neph & TL newhy Neph & TL newhv Neph & TL Neph & TL Neph & TL Neph & TL Neph \\
\hline 1 & Case 15B - Batch 2 & 51 & TL newhy Neph & TL newhy Neph & TL newhy Neph & TL Neph & TL Neph & TL Neph & TL Neph \\
\hline 1 & Case 15B - Batch 2 & 52 & TL newhy Neph & TL newhy Neph & TL newhy Neph & TL Neph & TL Neph & TL Neph & TL Neph \\
\hline 1 & Case 15B - Batch 2 & 53 & TL newhy Neph & TL newhy Neph & TL Neph & TL Neph & TL Neph & TL Neph & TL Neph \\
\hline 1 & Case 15B - Batch 2 & 54 & TL newhy Neph & TL newhy Neph & TL Neph & TL Neph & TL Neph & TL Neph & TL Neph \\
\hline 1 & Case 15B - Batch 2 & 55 & TL newhy Neph & TL newhy Neph & TL Neph & TL Neph & TL Neph & TL Neph & TL Neph \\
\hline 1 & Case 15B - Batch 2 & 56 & TL newhy Neph & TL newhy Neph & TL Neph & TL Neph & TL Neph & TL Neph & TL Neph \\
\hline 1 & Case 15B - Batch 2 & 57 & TL newhv Neph & TL newhv Neph & TL Neph & TL Neph & TL Neph & TL Neph & TL Neph \\
\hline 1 & Case 15B - Batch 2 & 58 & TL newhy Neph & TL Neph & TL Neph & TL Neph & TL Neph & TL Neph & TL Neph \\
\hline 1 & Case 15B - Batch 2 & 59 & TL newhy Neph & TL Neph & TL Neph & TL Neph & TL Neph & TL newlv Neph & TL Neph \\
\hline 1 & Case 15B - Batch 2 & 60 & TL newhv Neph & TL Neph & TL Neph & TL Neph & TL Neph & TL newlv Neph & TL Neph \\
\hline 1 & Case 15B - Blend 1 & 25 & newhv & newhy & newhv & & & & \\
\hline 1 & Case 15B - Blend 1 & 26 & newhy & newhy & newhv & & & & \\
\hline 1 & Case 15B - Blend 1 & 27 & newhv & newhv & & & & & \\
\hline 1 & Case 15B - Blend 1 & 28 & newhv & newhy & & & & & \\
\hline 1 & Case 15B - Blend 1 & 29 & newhv & newhv & & & & & \\
\hline 1 & Case 15B - Blend 1 & 30 & newhy & newhy & & & & & \\
\hline 1 & Case 15B - Blend 1 & 31 & newhv & & & & & & \\
\hline 1 & Case 15B - Blend 1 & 32 & newhv & & & & & newlv & \\
\hline 1 & Case 15B - Blend 1 & 33 & newhv & & & & & newlv & \\
\hline 1 & $\begin{array}{l}\text { Case } 15 \mathrm{~B} \text { - Blend } 1 \\
\end{array}$ & 34 & & & & & & newlv & \\
\hline 1 & Case 15B - Blend 1 & 35 & & & & & & newlv & \\
\hline 1 & Case 15B - Blend 1 & 36 & & & & & & newlv & \\
\hline 1 & Case 15B - Blend 1 & 37 & & & & & & newlv & \\
\hline 1 & Case 15B - Blend 1 & 38 & & & & & & newlv & \\
\hline 1 & Case 15B - Blend 1 & 39 & & & & & newlv & newlv & \\
\hline 1 & Case 15B - Blend 1 & 40 & & & & $\mathrm{TL}$ & newlv & newlv & \\
\hline 1 & Case 15B - Blend 1 & 41 & & & & $\mathrm{TL}$ & newlv & newlv & \\
\hline 1 & Case 15B - Blend 1 & 42 & $\mathrm{TL}$ & & & $\mathrm{TL}$ & newlv & newlv & newlv \\
\hline 1 & Case 15B - Blend 1 & 43 & $\mathrm{TL}$ & & & $\mathrm{TL}$ & TL newlv & TL newlv & newlv \\
\hline 1 & Case 15B - Blend 1 & 44 & $\mathrm{TL}$ & $\mathrm{TL}$ & & $\mathrm{TL}$ & TL newlv & TL newlv & newlv \\
\hline 1 & $\begin{array}{l}\text { Case } 15 \mathrm{~B} \text { - Blend } 1 \\
\end{array}$ & 45 & $\mathrm{TL}$ & $\mathrm{TL}$ & $\mathrm{TL}$ & $\mathrm{TL}$ & TL newlv & TL newlv & newlv \\
\hline 1 & Case 15B - Blend 1 & 46 & $\mathrm{TL}$ & $\mathrm{TL}$ & $\mathrm{TL}$ & $\mathrm{TL}$ & TL newlv & TL newlv & newlv \\
\hline 1 & Case 15B - Blend 1 & 47 & $\mathrm{TL}$ & $\mathrm{TL}$ & $\mathrm{TL}$ & $\mathrm{TL}$ & TL newlv & TL newlv & newlv \\
\hline 1 & Case 15B - Blend 1 & 48 & $\mathrm{TL}$ & $\mathrm{TL}$ & $\mathrm{TL}$ & TL newlv & TL newlv & TL newlv & TL newlv \\
\hline 1 & Case 15B - Blend 1 & 49 & $\mathrm{TL}$ & $\mathrm{TL}$ & $\mathrm{TL}$ & TL newlv & TL newlv & TL newlv & TL newlv \\
\hline 1 & Case 15B - Blend 1 & 50 & $\mathrm{TL}$ & $\mathrm{TL}$ & $\mathrm{TL}$ & TL newlv & TL newlv & TL newlv Neph & TL newlv \\
\hline 1 & Case 15B - Blend 1 & 51 & $\mathrm{TL}$ & $\mathrm{TL}$ & $\mathrm{TL}$ & TL newlv & TL newlv Neph & TL newlv Neph & TL newlv \\
\hline 1 & Case 15B - Blend 1 & 52 & $\mathrm{TL}$ & $\mathrm{TL}$ & TL & TL newlv Neph & TL newlv Neph & TL newlv Neph & TL newlv Neph \\
\hline 1 & Case 15B - Blend 1 & 53 & $\mathrm{TL}$ & $\mathrm{TL}$ & TL newlv Neph & TL newlv Neph & TL newlv Neph & TL newlv Neph & TL newlv Neph \\
\hline 1 & Case 15B - Blend 1 & 54 & $\mathrm{TL}$ & TL newlv Neph & TL newlv Neph & TL newlv Neph & TL newlv Neph & TL newlv Neph & TL newlv Neph \\
\hline 1 & Case 15B - Blend 1 & 55 & TL Neph & TL newlv Neph & TL newlv Neph & TL newlv Neph & TL newlv Neph & TL newlv Neph & TL newlv Neph \\
\hline 1 & Case 15B - Blend 1 & 56 & TL newlv Neph & TL newlv Neph & TL newlv Neph & TL newlv Neph & TL newlv Neph & TL newlv Neph & TL newlv Neph \\
\hline
\end{tabular}


Exhibit A1. Results for MAR Assessments of the Nominal Stage (part 1)

Constraint not met: neph (nepheline), TL (liquidus temperature), newlv (low viscosity - new model), newhv (high viscosity - new model), and Del GP - durability

\begin{tabular}{|c|c|c|c|c|c|c|c|c|c|}
\hline & & $\begin{array}{l}\mathrm{Na} 2 \mathrm{O} \\
\end{array}$ & 0.03 & 0.04 & 0.05 & 0.05 & 0.05 & 0.05 & 0.05 \\
\hline Set & Tyрe & Sludge Loading (\%) & 422 & $\mathrm{~d} 1-1$ & 473 & P1-1 & P2-1 & P2-2 & P3-1 \\
\hline 1 & Case 15B - Blend 1 & 57 & TL newlv Neph & TL newlv Neph & TL newlv Neph & TL newlv Neph & TL newlv Neph & TL newlv Neph & TL newlv Neph \\
\hline 1 & Case 15B - Blend 1 & 58 & TL newlv Neph & TL newlv Neph & TL newlv Neph & TL newlv Neph & TL newlv Neph & TL newlv Neph & TL newlv Neph \\
\hline 1 & Case 15B - Blend 1 & 59 & TL newlv Neph & TL newlv Neph & TL newlv Neph & TL newlv Neph & TL newlv Neph & TL newlv Neph & TL newlv Neph \\
\hline 1 & Case 15B - Blend 1 & 60 & TL newlv Neph & TL newlv Neph & TL newlv Neph & TL newlv Neph & TL newlv Neph & TL newlv Neph & TL newlv Neph \\
\hline 1 & Case 15B - Blend 2 & 25 & newhv & newhv & newhv & newhy & & & \\
\hline 1 & Case 15B - Blend 2 & 26 & newhv & newhv & newhv & newhv & & & \\
\hline 1 & Case $15 \mathrm{~B}$ - Blend 2 & 27 & newhv & newhv & newhy & newhy & & & \\
\hline 1 & Case 15B - Blend 2 & 28 & newhy & newhy & newhy & newhy & & & \\
\hline 1 & Case 15B - Blend 2 & 29 & newhv & newhv & newhv & newhv & & & \\
\hline 1 & Case $15 \mathrm{~B}$ - Blend 2 & 30 & newhy & newhy & newhy & newhy & & & \\
\hline$\frac{1}{1}$ & Case 15B - Blend 2 & 31 & newhy & newhy & newhy & & & & \\
\hline 1 & Case 15B - Blend 2 & 32 & newhv & newhy & newhv & & & & \\
\hline 1 & Case 15B - Blend 2 & 33 & newhv & newhv & newhy & & & & \\
\hline$\frac{1}{1}$ & Case 15B - Blend 2 & 34 & newhy & newhy & newhy & & & & \\
\hline 1 & Case 15B - Blend 2 & 35 & newhy & newhy & newhy & & & & \\
\hline 1 & Case 15B - Blend 2 & 36 & newhv & newhv & newhy & TL & & & \\
\hline 1 & Case 15B - Blend 2 & 37 & newhy & newhv & newhy & $\mathrm{TL}$ & & & \\
\hline$\frac{1}{1}$ & Case 15B - Blend 2 & 38 & newhv & newhy & newhy & $\frac{T L}{T L}$ & & & \\
\hline 1 & Case 15B - Blend 2 & 39 & TL newhv & newhy & newhv & $\mathrm{TL}$ & $\mathrm{TL}$ & $\mathrm{TL}$ & \\
\hline 1 & Case 15B - Blend 2 & 40 & TL newhy & TL newhy & newhv & $\mathrm{TL}$ & $\mathrm{TL}$ & $\mathrm{TL}$ & \\
\hline 1 & Case 15B - Blend 2 & 41 & TL newhv & TL newhv & TL newhv & $\mathrm{TL}$ & $\mathrm{TL}$ & $\mathrm{TL}$ & \\
\hline 1 & Case $15 \mathrm{~B}$ - Blend 2 & 42 & TL newhv & TL newhy & TL newhy & $\mathrm{TL}$ & $\mathrm{TL}$ & $\mathrm{TL}$ & \\
\hline 1 & Case 15B - Blend 2 & 43 & TL newhv & TL newhy & TL newhv & $\mathrm{TL}$ & $\mathrm{TL}$ & $\mathrm{TL}$ & \\
\hline 1 & Case 15B - Blend 2 & 44 & TL newhy & TL newhy & $\mathrm{TL}$ & $\mathrm{TL}$ & $\mathrm{TL}$ & $\mathrm{TL}$ & $\mathrm{TL}$ \\
\hline$\frac{1}{1}$ & Case 15B - Blend 2 & 45 & TL newhy & TL newhy & $\mathrm{TL}$ & $\mathrm{TL}$ & $\mathrm{TL}$ & TL Neph & $\mathrm{TL}$ \\
\hline 1 & Case 15B - Blend 2 & 46 & TL newhv & TL newhy & $\mathrm{TL}$ & TL Neph & TL Neph & TL Neph & $\mathrm{TL}$ \\
\hline 1 & Case $15 \mathrm{~B}-$ Blend 2 & 47 & TL newhv & TL newhv & $\mathrm{TL}$ & TL Neph & TL Neph & TL Neph & TL Neph \\
\hline$\frac{1}{1}$ & Case 15B - Blend 2 & 48 & TL newhy & TL & TL Neph & TL Neph & TL Neph & TL Neph & TL Neph \\
\hline 1 & Case 15B - Blend 2 & 49 & TL newhy & TL Neph & TL Neph & TL Neph & TL Neph & TL Neph & TL Neph \\
\hline 1 & Case 15B - Blend 2 & 50 & TL newhv Neph & TL Neph & TL Neph & TL Neph & TL Neph & TL newlv Neph & TL Neph \\
\hline 1 & Case 15B - Blend 2 & 51 & TL newhv Neph & TL Neph & TL Neph & TL Neph & TL Neph & TL newlv Neph & TL Neph \\
\hline 1 & Case 15B - Blend 2 & 52 & TL Neph & TL Neph & TL Neph & TL Neph & TL Neph & TL newlv Neph & TL Neph \\
\hline 1 & Case 15B - Blend 2 & 53 & TL Neph & TL Neph & TL Neph & TL Neph & TL Neph & TL newlv Neph & TL Neph \\
\hline 1 & Case 15B - Blend 2 & 54 & TL Neph & TL Neph & TL Neph & TL Neph & TL Neph & TL newlv Neph & TL Neph \\
\hline 1 & Case 15B - Blend 2 & 55 & TL Neph & TL Neph & TL Neph & TL Neph & TL Neph & TL newlv Neph & TL Neph \\
\hline$\frac{1}{1}$ & Case 15B - Blend 2 & 56 & TL Neph & TL Neph & TL Neph & TL Neph & TL Neph & TL newlv Neph & TL Neph \\
\hline 1 & Case 15B - Blend 2 & 57 & TL Neph & TL Neph & TL Neph & TL Neph & TL Neph & TL newlv Neph & TL Neph \\
\hline 1 & Case 15B - Blend 2 & 58 & TL Neph & TL Neph & TL Neph & TL Neph & TL newlv Neph & TL newlv Neph & TL Neph \\
\hline 1 & Case 15B - Blend 2 & 59 & TL Neph & TL Neph & TL Neph & TL Neph & TL newlv Neph & TL newlv Neph & TL Neph \\
\hline 1 & Case 15B - Blend 2 & 60 & TL Neph & TL Neph & TL Neph & TL Neph & TL newlv Neph & TL newlv Neph & TL newlv Neph \\
\hline 1 & Case 16 - Batch 1 & 25 & $\mathrm{Al} 2 \mathrm{O} 3$ & $\mathrm{Al} 2 \mathrm{O} 3$ & $\mathrm{Al} 2 \mathrm{O} 3$ & $\mathrm{Al} 2 \mathrm{O} 3$ & $\mathrm{Al} 2 \mathrm{O} 3 \mathrm{R} 2 \mathrm{O}$ & newlv Al2O3 R2O & $\mathrm{Al} 2 \mathrm{O} 3 \mathrm{R} 2 \mathrm{O}$ \\
\hline 1 & Case 16 - Batch 1 & 26 & $\mathrm{Al} 2 \mathrm{O} 3$ & $\mathrm{Al} 2 \mathrm{O} 3$ & $\mathrm{Al} 2 \mathrm{O} 3$ & $\mathrm{Al} 2 \mathrm{O} 3$ & $\mathrm{Al} 2 \mathrm{O} 3 \mathrm{R} 2 \mathrm{O}$ & Del Gp newlv Al2O3 R2O & Del Gp Al2O3 R2O \\
\hline$\frac{1}{1}$ & Case 16 - Batch 1 & 27 & $\mathrm{Al} 2 \mathrm{O} 3$ & $\mathrm{Al} 2 \mathrm{O} 3$ & $\mathrm{Al} 2 \mathrm{O} 3$ & $\mathrm{Al} 2 \mathrm{O} 3$ & newlv Al2O3 R2O & Del Gp newlv Al2O3 R2O & Del Gp Al2O3 R2O \\
\hline 1 & Case 16 - Batch 1 & 28 & $\mathrm{Al} 2 \mathrm{O} 3$ & $\mathrm{Al} 2 \mathrm{O} 3$ & $\mathrm{Al} 2 \mathrm{O} 3$ & $\mathrm{Al} 2 \mathrm{O} 3$ & newlv Al2O3 R2O & Del Gp newlv Al2O3 R2O & Del Gp Al2O3 R2O \\
\hline 1 & Case 16 - Batch 1 & 29 & $\mathrm{Al} 2 \mathrm{O} 3$ & $\mathrm{Al} 2 \mathrm{O} 3$ & $\mathrm{Al} 2 \mathrm{O} 3$ & $\mathrm{Al} 2 \mathrm{O} 3$ & newlv Al2O3 R2O & Del Gp newlv Al2O3 R2O & Del Gp newlv Al2O3 R2O \\
\hline 1 & Case 16 - Batch 1 & 30 & $\mathrm{Al} 2 \mathrm{O} 3$ & $\mathrm{Al} 2 \mathrm{O} 3$ & $\mathrm{Al} 2 \mathrm{O} 3$ & $\mathrm{Al} 2 \mathrm{O} 3$ & Del Gp newlv Al2O3 R2O & Del Gp newlv Al2O3 R2O & Del Gp newlv Al2O3 R2O \\
\hline 1 & Case 16 - Batch 1 & 31 & $\mathrm{Al} 2 \mathrm{O} 3$ & $\mathrm{Al} 2 \mathrm{O} 3$ & $\mathrm{Al} 2 \mathrm{O} 3 \mathrm{R} 2 \mathrm{O}$ & $\mathrm{Al} 2 \mathrm{O} 3 \mathrm{R} 2 \mathrm{O}$ & Del Gp newlv Al2O3 R2O & Del Gp newlv Al2O3 R2O & Del Gp newlv Al2O3 R2O \\
\hline 1 & Case 16 - Batch 1 & 32 & $\mathrm{Al} 2 \mathrm{O} 3$ & $\mathrm{Al} 2 \mathrm{O} 3$ & $\mathrm{Al} 2 \mathrm{O} 3 \mathrm{R} 2 \mathrm{O}$ & $\mathrm{Al} 2 \mathrm{O} 3 \mathrm{R} 2 \mathrm{O}$ & Del Gp newlv Al2O3 R2O & Del Gp newlv Al2O3 R2O & Del Gp newlv Al2O3 R2O \\
\hline 1 & Case 16 - Batch 1 & 33 & $\mathrm{Al} 2 \mathrm{O} 3$ & $\mathrm{Al} 2 \mathrm{O} 3$ & $\mathrm{Al} 2 \mathrm{O} 3 \mathrm{R} 2 \mathrm{O}$ & $\mathrm{Al} 2 \mathrm{O} 3 \mathrm{R} 2 \mathrm{O}$ & Del Gp newlv Al2O3 R2O & Del Gp newlv Al2O3 R2O & Del Gp newlv Al2O3 R2O \\
\hline 1 & Case 16 - Batch 1 & 34 & $\mathrm{Al} 2 \mathrm{O} 3$ & $\mathrm{Al} 2 \mathrm{O} 3$ & $\mathrm{Al} 2 \mathrm{O} 3 \mathrm{R} 2 \mathrm{O}$ & newlv Al2O3 R2O & Del Gp newlv Al2O3 R2O & Del Gp newlv Al2O3 R2O & Del Gp newlv Al2O3 R2O \\
\hline 1 & Case 16 - Batch 1 & 35 & $\mathrm{Al} 2 \mathrm{O} 3$ & $\mathrm{Al} 2 \mathrm{O} 3 \mathrm{R} 2 \mathrm{O}$ & $\mathrm{Al} 2 \mathrm{O} 3 \mathrm{R} 2 \mathrm{O}$ & newlv Al2O3 R2O & Del Gp newlv Al2O3 R2O & Del Gp newlv Al2O3 R2O & Del Gp newlv Al2O3 R2O \\
\hline 1 & Case 16 - Batch 1 & 36 & $\mathrm{Al} 2 \mathrm{O} 3$ & $\mathrm{Al} 2 \mathrm{O} 3 \mathrm{R} 2 \mathrm{O}$ & Del Gp Al2O3 R2O & Del Gp newlv Al2O3 R2O & Del Gp newlv Al2O3 R2O & Del Gp newlv Al2O3 R2O & Del Gp newlv Al2O3 R2O \\
\hline
\end{tabular}


Exhibit A1. Results for MAR Assessments of the Nominal Stage (part 1)

Constraint not met: neph (nepheline), TL (liquidus temperature), newlv (low viscosity - new model), newhv (high viscosity - new model), and Del GP - durability

\begin{tabular}{|c|c|c|c|c|c|c|c|c|c|}
\hline & & $\mathrm{Na} 2 \mathrm{O}$ & 0.03 & 0.04 & 0.05 & 0.05 & 0.05 & 0.05 & 0.05 \\
\hline Set & Type & $\begin{array}{l}\text { Sludge Loading }(\%) \\
\end{array}$ & 422 & $\mathrm{~d} 1-1$ & 473 & P1-1 & P2-1 & P2-2 & P3-1 \\
\hline 1 & Case 16 - Batch 1 & 37 & $\mathrm{Al} 2 \mathrm{O} 3$ & $\begin{array}{l}\mathrm{Al} 2 \mathrm{O} 3 \mathrm{R} 2 \mathrm{O} \\
\end{array}$ & Del Gp Al2O3 R2O & Del Gp newlv Al2O3 R2O & Del Gp newlv Al2O3 R2O & Del Gp newlv Al2O3 R2O & Del Gp newlv Al2O3 R2O \\
\hline 1 & Case 16 - Batch 1 & 38 & Al2O3 R2O & $\mathrm{Al} 2 \mathrm{O} 3 \mathrm{R} 2 \mathrm{O}$ & Del Gp newlv Al2O3 R2O & Del Gp newlv Al2O3 R2O & Del Gp newlv Al2O3 R2O & Del Gp newlv Al2O3 R2O & Del Gp newlv Al2O3 R2O \\
\hline 1 & Case 16 - Batch 1 & 39 & $\mathrm{Al} 2 \mathrm{O} 3 \mathrm{R} 2 \mathrm{O}$ & Del Gp Al2O3 R2O & Del Gp newlv Al2O3 R2O & Del Gp newlv Al2O3 R2O & Del Gp newlv Al2O3 R2O & Del Gp newlv Al2O3 R2O & Del Gp newlv Al2O3 R2O \\
\hline 1 & Case 16 - Batch 1 & 40 & $\mathrm{Al} 2 \mathrm{O} 3 \mathrm{R} 2 \mathrm{O}$ & Del Gp newlv Al2O3 R2O & Del Gp newlv Al2O3 R2O & Del Gp newlv Al2O3 R2O & Del Gp newlv Al2O3 R2O & Del Gp newlv Al2O3 R2O & Del Gp newlv Al2O3 R2O \\
\hline 1 & Case 16 - Batch 1 & 41 & Del Gp newlv Al2O3 R2O & Del Gp newlv Al2O3 R2O & Del Gp newlv Al2O3 R2O & Del Gp newlv Al2O3 R2O & Del Gp newlv Al2O3 R2O & Del Gp newlv Al2O3 R2O & Del Gp newlv Al2O3 R2O \\
\hline 1 & Case 16 - Batch 1 & 42 & Del Gp newlv Al2O3 R2O & Del Gp newlv Al2O3 R2O & Del Gp newlv Al2O3 R2O & Del Gp newlv Al2O3 R2O & Del Gp newlv Al2O3 R2O & Del Gp newlv Al2O3 R2O & Del Gp newlv Al2O3 R2O \\
\hline 1 & Case 16 - Batch 1 & 43 & Del Gp newlv Al2O3 R2O & Del Gp newlv Al2O3 R2O & Del Gp newlv Al2O3 R2O & Del Gp newlv Al2O3 R2O & Del Gp newlv Al2O3 R2O & Del Gp newlv Al2O3 R2O & Del Gp newlv Al2O3 R2O \\
\hline 1 & Case 16 - Batch 1 & 44 & Del Gp newlv Al2O3 R2O & Del Gp newlv Al2O3 R2O & Del Gp newlv Al2O3 R2O & Del Gp newlv Al2O3 R2O & Del Gp newlv Al2O3 R2O & Del Gp newlv Al2O3 R2O & Del Gp newlv Al2O3 R2O \\
\hline 1 & Case 16 - Batch 1 & 45 & Del Gp newlv Al2O3 R2O & Del Gp newlv Al2O3 R2O & Del Gp newlv Al2O3 R2O & Del Gp newlv Al2O3 R2O & Del Gp newlv Al2O3 R2O & Del Gp newlv Al2O3 R2O & Del Gp newlv Al203 R2O \\
\hline 1 & Case 16 - Batch 1 & 46 & Del Gp newlv Al2O3 R2O & Del Gp newlv Al2O3 R2O & Del Gp newlv Al2O3 R2O & Del Gp newlv Al2O3 R2O & Del Gp newlv Al2O3 R2O & Del Gp newlv Al2O3 R2O & Del Gp newlv Al2O3 R2O \\
\hline 1 & Case 16 - Batch 1 & 47 & Del Gp newlv Al2O3 R2O & Del Gp newlv Al2O3 R2O & Del Gp newlv Al2O3 R2O & Del Gp newlv Al2O3 R2O & Del Gp newlv Al2O3 R2O & Del Gp newlv Al2O3 R2O & Del Gp newlv Al2O3 R2O \\
\hline 1 & Case 16 - Batch 1 & 48 & Del Gp newlv Al2O3 R2O & Del Gp newlv Al2O3 R2O & Del Gp newlv Al2O3 R2O & Del Gp newlv Al2O3 R2O & Del Gp newlv Al2O3 R2O & Del Gp newlv Al2O3 R2O & Del Gp newlv Al2O3 R2O \\
\hline 1 & Case 16 - Batch 1 & 49 & Del Gp newlv Al2O3 R2O & Del Gp newlv Al2O3 R2O & Del Gp newlv Al2O3 R2O & Del Gp newlv Al2O3 R2O & Del Gp newlv Al2O3 R2O & Del Gp newlv Al2O3 R2O & Del Gp newlv Al2O3 R2O \\
\hline 1 & Case 16 - Batch 1 & 50 & Del Gp newlv Al2O3 R2O & Del Gp newlv Al2O3 R2O & Del Gp newlv Al2O3 R2O & Del Gp newlv Al2O3 R2O & Del Gp newlv Al2O3 R2O & Del Gp newlv Al2O3 R2O & Del Gp newlv Al2O3 R2O \\
\hline 1 & Case 16 - Batch 1 & 51 & Del Gp newlv R2O & Del Gp newlv R2O & Del Gp newlv R2O & Del Gp newlv R2O & Del Gp newlv R2O & Del Gp newlv R2O & Del Gp newlv R2O \\
\hline 1 & Case 16 - Batch 1 & 52 & Del Gp newlv R2O & Del Gp newlv R2O & Del Gp newlv R2O & Del Gp newlv R2O & Del Gp newlv R2O & Del Gp newlv Neph & Del Gp newlv R2O \\
\hline 1 & Case 16 - Batch 1 & 53 & Del Gp newlv R2O & Del Gp newlv R2O & Del Gp newlv R2O & Del Gp TL newlv R2O Neph & Del Gp newlv R2O Neph & Del Gp newlv Neph & Del Gp newlv R2O \\
\hline 1 & Case 16 - Batch 1 & 54 & Del Gp TL newlv R2O & Del Gp newlv R2O & Del Gp newlv R2O & Del Gp TL newlv R2O Neph & Del Gp newlv R2O Neph & Del Gp newlv Neph & Del Gp newlv R2O Neph \\
\hline 1 & Case 16 - Batch 1 & 55 & Del Gp TL newlv R2O & Del Gp TL newlv R2O & Del Gp newlv R2O Neph & Del Gp TL newlv Neph & Del Gp newlv Neph & Del Gp newlv Neph & Del Gp newlv R2O Neph \\
\hline 1 & Case 16 - Batch 1 & 56 & Del Gp TL newlv R2O & Del Gp TL newlv R2O Neph & Del Gp newlv R2O Neph & Del Gp TL newlv Neph & Del Gp newlv Neph & Del Gp newly Neph & Del Gp newlv R2O Neph \\
\hline 1 & Case 16 - Batch 1 & 57 & Del Gp TL newlv R2O Neph & Del Gp TL newlv R2O Neph & Del Gp TL newlv R2O Neph & Del Gp TL newlv Neph & Del Gp TL newlv Neph & Del Gp TL newlv Neph & Del Gp newlv R2O Neph \\
\hline 1 & Case 16 - Batch 1 & 58 & Del Gp TL newlv R2O Neph & Del Gp TL newlv R2O Neph & Del Gp TL newlv R2O Neph & Del Gp TL newlv Neph & Del Gp TL newlv Neph & Del Gp TL newlv Neph & Del Gp newlv R2O Neph \\
\hline 1 & Case 16 - Batch 1 & 59 & Del Gp TL newlv R2O Neph & Del Gp TL newlv R2O Neph & Del Gp TL newlv R2O Neph & Del Gp TL newlv Neph & Del Gp TL newlv Neph & Del Gp TL newlv Neph & Del Gp newlv R2O Neph \\
\hline 1 & Case 16 - Batch 1 & 60 & Del Gp TL newlv R2O Neph & Del Gp TL newlv R2O Neph & Del Gp TL newlv R2O Neph & Del Gp TL newlv Neph & Del Gp TL newlv Neph & Del Gp TL newlv Neph & Del Gp newlv R2O Neph \\
\hline 1 & Case 16 - Batch 2 & 25 & newhv & newhv & newhv & newhv & & & \\
\hline 1 & Case 16 - Batch 2 & 26 & newhv & newhv & newhy & newhy & & & \\
\hline 1 & Case 16 - Batch 2 & 27 & newhv & newhy & newhy & newhy & & & \\
\hline 1 & Case 16 - Batch 2 & 28 & newhv & newhv & newhy & newhy & & & \\
\hline 1 & Case 16 - Batch 2 & 29 & newhv & newhv & newhy & newhy & & & \\
\hline 1 & Case 16 - Batch 2 & 30 & newhy & newhy & newhy & newhy & & & \\
\hline 1 & Case 16 - Batch 2 & 31 & newhy & newhy & newhv & newhv & & & \\
\hline 1 & Case 16 - Batch 2 & 32 & newhv & newhv & newhy & newhy & & & \\
\hline 1 & Case 16 - Batch 2 & 33 & newhv & newhv & newhv & newhy & & & \\
\hline 1 & Case 16 - Batch 2 & 34 & newhv & newhv & newhy & newhy & & & \\
\hline 1 & Case 16 - Batch 2 & 35 & newhv & newhv & newhy & newhy & & & \\
\hline 1 & Case 16 - Batch 2 & 36 & newhv & newhv & newhy & newhy & & & \\
\hline 1 & Case 16 - Batch 2 & 37 & newhv & newhv & newhy & newhv & & & \\
\hline 1 & Case 16 - Batch 2 & 38 & newhv & newhv & newhy & newhy & & & \\
\hline 1 & Case 16 - Batch 2 & 39 & newhv & newhv & newhy & newhy & & & \\
\hline 1 & Case 16 - Batch 2 & 40 & newhv & newhv & newhv & newhv & & & \\
\hline 1 & Case 16 - Batch 2 & 41 & newhy & newhy & newhy & newhy & & Neph & \\
\hline 1 & Case 16 - Batch 2 & 42 & newhv & newhv & newhv & newhv Neph & Neph & Neph & \\
\hline 1 & Case 16 - Batch 2 & 43 & newhv & newhv & newhy & newhv Neph & Neph & Neph & Neph \\
\hline 1 & Case 16 - Batch 2 & 44 & newhy & newhv & newhv Neph & TL newhy Neph & Neph & Neph & Neph \\
\hline 1 & Case 16 - Batch 2 & 45 & newhv Neph & newhv Neph & newhv Neph & TL newhy Neph & Neph & Neph & Neph \\
\hline 1 & Case 16 - Batch 2 & 46 & newhv Neph & newhv Neph & newhy Neph & TL newhy Neph & TL Neph & TL Neph & Neph \\
\hline 1 & Case 16 - Batch 2 & 47 & newhv Neph & newhv Neph & newhy Neph & TL Neph & TL Neph & TL Neph & Neph \\
\hline 1 & Case 16 - Batch 2 & 48 & TL newhy Neph & newhv Neph & newhy Neph & TL Neph & TL Neph & TL Neph & Neph \\
\hline 1 & Case 16 - Batch 2 & 49 & TL newhv Neph & TL newhv Neph & newhv Neph & TL Neph & TL Neph & TL Neph & Neph \\
\hline 1 & Case 16 - Batch 2 & 50 & TL newhv Neph & TL newhy Neph & TL newhy Neph & TL Neph & TL Neph & TL Neph & Neph \\
\hline 1 & Case 16 - Batch 2 & 51 & TL newhy Neph & TL newhy Neph & TL newhy Neph & TL Neph & TL Neph & TL Neph & Neph \\
\hline 1 & Case 16 - Batch 2 & 52 & TL newhv Neph & TL n newhy Neph & TL newhy Neph & TL Neph & TL Neph & TL Neph & Neph \\
\hline
\end{tabular}


Exhibit A1. Results for MAR Assessments of the Nominal Stage (part 1)

Constraint not met: neph (nepheline), TL (liquidus temperature), newlv (low viscosity - new model), newhv (high viscosity - new model), and Del GP - durability

\begin{tabular}{|c|c|c|c|c|c|c|c|c|c|}
\hline & & $\mathrm{Na} 2 \mathrm{O}$ & 0.03 & 0.04 & 0.05 & 0.05 & 0.05 & 0.05 & 0.05 \\
\hline Set & $\begin{array}{c}\text { Type } \\
\end{array}$ & $\begin{array}{l}\text { Sludge Loading }(\%) \\
\end{array}$ & 422 & $\mathrm{~d} 1-1$ & 473 & P1-1 & $\mathrm{P} 2-1$ & P2-2 & P3-1 \\
\hline 1 & Case 16 - Batch 2 & 53 & TL newhy Neph & TL newhy Neph & TL newhv Neph & TL Neph & TL Neph & TL Neph & TL Neph \\
\hline 1 & Case 16 - Batch 2 & 54 & TL newhy Neph & TL newhy Neph & TL newhy Neph & TL Neph & TL Neph & TL Neph & TL Neph \\
\hline 1 & $\begin{array}{l}\text { Case } 16 \text { - Batch } 2 \\
\end{array}$ & 55 & TL newhy Neph & TL newhy Neph & TL newhy Neph & TL Neph & TL Neph & TL Neph & TL Neph \\
\hline 1 & Case 16 - Batch 2 & 56 & TL newhy Neph & TL newhy Neph & TL newhy Neph & TL Neph & TL Neph & TL Neph & TL Neph \\
\hline 1 & Case 16 - Batch 2 & 57 & TL newhv Neph & TL newhv Neph & TL newhv Neph & TL Neph & TL Neph & TL Neph & TL Neph \\
\hline 1 & Case 16 - Batch 2 & 58 & TL newhy Neph & TL newhy Neph & TL newhy Neph & TL Neph & TL Neph & TL Neph & TL Neph \\
\hline 1 & Case 16 - Batch 2 & 59 & TL newhy Neph & TL newhy Neph & TL newhy Neph & TL Neph & TL Neph & TL Neph & TL Neph \\
\hline 1 & Case 16 - Batch 2 & 60 & TL newhy Neph & TL newhy Neph & TL newhy Neph & TL Neph & TL Neph & TL Neph & TL Neph \\
\hline 1 & Case 16 - Blend 1 & 25 & newhy & newhv & newhy & & & & \\
\hline 1 & Case 16 - Blend 1 & 26 & newhv & newhy & & & & & \\
\hline 1 & Case 16 - Blend 1 & 27 & newhv & newhy & & & & & \\
\hline 1 & Case 16 - Blend 1 & 28 & newhv & newhv & & & & & \\
\hline 1 & Case 16 - Blend 1 & 29 & newhy & & & & & & \\
\hline 1 & Case 16 - Blend 1 & 30 & newhv & & & & & newlv & \\
\hline 1 & Case 16 - Blend 1 & 31 & newhv & & & & & newlv & \\
\hline 1 & Case 16 - Blend 1 & 32 & & & & & & newlv & \\
\hline 1 & Case 16 - Blend 1 & 33 & & & & & & newlv & \\
\hline 1 & Case 16 - Blend 1 & 34 & & & & & & newlv & \\
\hline 1 & Case 16 - Blend 1 & 35 & & & & $\mathrm{TL}$ & & newlv & \\
\hline 1 & Case 16 - Blend 1 & 36 & $\mathrm{TL}$ & & & $\mathrm{TL}$ & & newlv & \\
\hline 1 & Case 16 - Blend 1 & 37 & $\mathrm{TL}$ & $\mathrm{TL}$ & & $\mathrm{TL}$ & TL newlv & TL newlv & \\
\hline 1 & Case 16 - Blend 1 & 38 & $\mathrm{TL}$ & $\mathrm{TL}$ & & $\mathrm{TL}$ & TL newlv & TL newlv & \\
\hline 1 & Case 16 - Blend 1 & 39 & $\mathrm{TL}$ & $\mathrm{TL}$ & $\mathrm{TL}$ & $\mathrm{TL}$ & TL newlv & TL newlv & \\
\hline 1 & Case 16 - Blend 1 & 40 & $\mathrm{TL}$ & $\mathrm{TL}$ & $\mathrm{TL}$ & $\mathrm{TL}$ & TL newlv & TL newlv & newlv \\
\hline 1 & Case 16 - Blend 1 & 41 & $\mathrm{TL}$ & $\mathrm{TL}$ & $\mathrm{TL}$ & $\mathrm{TL}$ & TL newlv & TL newlv & newlv \\
\hline 1 & Case 16 - Blend 1 & 42 & $\mathrm{TL}$ & $\mathrm{TL}$ & $\mathrm{TL}$ & $\mathrm{TL}$ & TL newlv & $\begin{array}{l}\text { TL newlv } \\
\end{array}$ & TL newlv \\
\hline 1 & Case 16 - Blend 1 & 43 & $\mathrm{TL}$ & $\mathrm{TL}$ & $\mathrm{TL}$ & $\mathrm{TL}$ & TL newlv & TL newlv & TL newlv \\
\hline 1 & Case 16 - Blend 1 & 44 & $\mathrm{TL}$ & $\mathrm{TL}$ & $\mathrm{TL}$ & $\mathrm{TL}$ & TL newlv & TL newlv & TL newlv \\
\hline 1 & Case 16 - Blend 1 & 45 & $\mathrm{TL}$ & $\mathrm{TL}$ & $\mathrm{TL}$ & TL & TL newlv & TL newlv & TL newlv \\
\hline 1 & Case 16 - Blend 1 & 46 & $\mathrm{TL}$ & $\mathrm{TL}$ & $\mathrm{TL}$ & TL newlv & TL newlv & TL newlv & TL newlv \\
\hline 1 & Case 16 - Blend 1 & 47 & $\mathrm{TL}$ & $\mathrm{TL}$ & $\mathrm{TL}$ & TL newlv & TL newlv & TL newlv & TL newlv \\
\hline 1 & Case 16 - Blend 1 & 48 & $\mathrm{TL}$ & $\mathrm{TL}$ & $\mathrm{TL}$ & TL newlv & TL newlv & TL newlv & TL newlv \\
\hline 1 & Case 16 - Blend 1 & 49 & $\mathrm{TL}$ & $\mathrm{TL}$ & $\mathrm{TL}$ & TL newlv & TL newlv & TL newlv & TL newlv \\
\hline 1 & Case 16 - Blend 1 & 50 & $\mathrm{TL}$ & $\mathrm{TL}$ & $\mathrm{TL}$ & TL newlv & TL newlv & TL newlv & TL newlv \\
\hline 1 & Case 16 - Blend 1 & 51 & $\mathrm{TL}$ & $\mathrm{TL}$ & TL newlv & TL newlv & TL newlv & TL newlv & TL newlv \\
\hline 1 & Case 16 - Blend 1 & 52 & $\mathrm{TL}$ & TL newlv & TL newlv & TL newlv & TL newlv & TL newlv & TL newlv \\
\hline 1 & Case 16 - Blend 1 & 53 & $\mathrm{TL}$ & TL newlv & TL newlv & TL newlv & TL newlv & TL newlv & TL newlv \\
\hline 1 & Case 16 - Blend 1 & 54 & TL newly & TL newlv & TL newlv & TL newlv & TL newlv & TL newlv & TL newlv \\
\hline 1 & Case 16 - Blend 1 & 55 & TL newlv & TL newlv & TL newlv & TL newlv & TL newlv & TL newlv Neph & TL newlv \\
\hline 1 & Case 16 - Blend 1 & 56 & TL newlv & TL newlv & $\begin{array}{l}\text { TL newlv } \\
\end{array}$ & TL newlv & TL newlv Neph & TL newlv Neph & TL newlv \\
\hline 1 & Case 16 - Blend 1 & 57 & TL newlv & TL newlv & TL newlv & TL newlv Neph & TL newlv Neph & TL newlv Neph & TL newlv Neph \\
\hline 1 & Case 16 - Blend 1 & 58 & TL newlv & TL newlv & TL newlv Neph & TL newlv Neph & TL newlv Neph & TL newlv Neph & TL newlv Neph \\
\hline 1 & Case 16 - Blend 1 & 59 & TL newlv & TL newlv Neph & TL newlv Neph & TL newlv Neph & TL newlv Neph & TL newlv Neph & TL newlv Neph \\
\hline 1 & Case 16 - Blend 1 & 60 & TL newlv Neph & TL newlv Neph & TL newlv Neph & TL newlv Neph & TL newlv Neph & TL newlv Neph & TL newlv Neph \\
\hline 1 & Case 16 - Blend 2 & 25 & newhv & newhv & newhv & newhv & & & \\
\hline 1 & Case 16 - Blend 2 & 26 & newhy & newhy & newhy & newhy & & & \\
\hline 1 & Case 16 - Blend 2 & 27 & newhv & newhv & newhy & newhy & & & \\
\hline 1 & Case 16 - Blend 2 & 28 & newhv & newhv & newhv & newhv & & & \\
\hline 1 & Case 16 - Blend 2 & 29 & newhv & newhv & newhy & newhy & & & \\
\hline 1 & Case 16 - Blend 2 & 30 & newhv & newhv & newhy & newhy & & & \\
\hline 1 & Case 16 - Blend 2 & 31 & newhv & newhv & newhy & newhy & & & \\
\hline 1 & Case 16 - Blend 2 & 32 & newhv & newhv & newhv & & & & \\
\hline
\end{tabular}


Exhibit A1. Results for MAR Assessments of the Nominal Stage (part 1)

Constraint not met: neph (nepheline), TL (liquidus temperature), newlv (low viscosity - new model), newhv (high viscosity - new model), and Del GP - durability

\begin{tabular}{|c|c|c|c|c|c|c|c|c|c|}
\hline & & $\mathrm{Na} 2 \mathrm{O}$ & 0.03 & 0.04 & 0.05 & 0.05 & 0.05 & 0.05 & 0.05 \\
\hline Set & Type & Sludge Loading (\%) & 422 & $\begin{array}{ll}\mathrm{d} 1-1 \\
\end{array}$ & 473 & P1-1 & P2-1 & P2-2 & P3-1 \\
\hline 1 & $\begin{array}{l}\text { Case } 16 \text { - Blend } 2 \\
\end{array}$ & 33 & newhv & newhv & newhv & & & & \\
\hline 1 & Case 16 - Blend 2 & 34 & newhy & newhy & newhy & & & & \\
\hline 1 & Case 16 - Blend 2 & 35 & newhv & newhv & newhy & & & & \\
\hline 1 & Case 16 - Blend 2 & 36 & newhy & newhv & newhy & & & & \\
\hline 1 & $\begin{array}{l}\text { Case } 16 \text { - Blend } 2 \\
\end{array}$ & 37 & newhv & newhv & newhv & & & & \\
\hline 1 & Case 16 - Blend 2 & 38 & newhv & newhv & newhv & & & & \\
\hline 1 & Case 16 - Blend 2 & 39 & newhv & newhv & newhy & & & & \\
\hline 1 & $\begin{array}{l}\text { Case } 16 \text { - Blend } 2 \\
\end{array}$ & 40 & newhv & newhv & newhy & $\mathrm{TL}$ & & & \\
\hline 1 & Case 16 - Blend 2 & 41 & newhy & newhy & newhy & $\mathrm{TL}$ & & & \\
\hline 1 & Case 16 - Blend 2 & 42 & newhv & newhv & newhy & $\mathrm{TL}$ & & $\mathrm{TL}$ & \\
\hline 1 & Case 16 - Blend 2 & 43 & TL newhy & newhy & newhy & $\mathrm{TL}$ & $\mathrm{TL}$ & TL Neph & \\
\hline 1 & Case 16 - Blend 2 & 44 & TL newhv & TL newhv & & $\mathrm{TL}$ & TL Neph & TL Neph & \\
\hline 1 & Case 16 - Blend 2 & 45 & TL newhy & TL newhy & $\mathrm{TL}$ & TL Neph & TL Neph & TL Neph & Neph \\
\hline 1 & Case 16 - Blend 2 & 46 & TL newhy & TL newhy & TL Neph & TL Neph & TL Neph & TL Neph & Neph \\
\hline 1 & $\begin{array}{l}\text { Case } 16 \text { - Blend } 2 \\
\end{array}$ & 47 & TL newhy & TL newhy Neph & TL Neph & TL Neph & TL Neph & TL Neph & Neph \\
\hline 1 & Case 16 - Blend 2 & 48 & TL newhy Neph & TL newhy Neph & TL Neph & TL Neph & TL Neph & TL Neph & TL Nepl \\
\hline 1 & Case 16 - Blend 2 & 49 & TL newhv Neph & TL Neph & TL Neph & TL Neph & TL Neph & TL Neph & TL Nepl \\
\hline 1 & $\begin{array}{l}\text { Case } 16 \text { - Blend } 2 \\
\end{array}$ & 50 & TL newhy Neph & TL Neph & TL Neph & TL Neph & TL Neph & TL Neph & TL Nepl \\
\hline 1 & Case 16 - Blend 2 & 51 & TL newhy Neph & TL Neph & TL Neph & TL Neph & TL Neph & TL newlv Neph & TL Nepl \\
\hline 1 & Case 16 - Blend 2 & 52 & TL newhv Neph & TL Neph & TL Neph & TL Neph & TL Neph & TL newlv Neph & TL Nepl \\
\hline 1 & Case 16 - Blend 2 & 53 & TL Neph & TL Neph & TL Neph & TL Neph & TL Neph & TL newlv Neph & TL Nepl \\
\hline 1 & Case 16 - Blend 2 & 54 & TL Neph & TL Neph & TL Neph & TL Neph & TL Neph & TL newlv Neph & TL Nepl \\
\hline 1 & $\begin{array}{l}\text { Case } 16-\text { Blend } 2 \\
\end{array}$ & 55 & TL Neph & TL Neph & TL Neph & TL Neph & TL Neph & TL newlv Neph & TL Nepl \\
\hline 1 & Case 16 - Blend 2 & 56 & TL Neph & TL Neph & TL Neph & TL Neph & TL Neph & TL newlv Neph & TL Nepl \\
\hline 1 & Case 16 - Blend 2 & 57 & TL Neph & TL Neph & TL Neph & TL Neph & TL Neph & TL newlv Neph & TL Nepl \\
\hline 1 & $\begin{array}{l}\text { Case } 16 \text { - Blend } 2 \\
\end{array}$ & 58 & TL Neph & TL Neph & TL Neph & TL Neph & TL Neph & TL newlv Neph & TL Nepl \\
\hline 1 & Case 16 - Blend 2 & 59 & TL Neph & TL Neph & TL Neph & TL Neph & TL newlv Neph & TL newlv Neph & TL Nepl \\
\hline 1 & Case 16 - Blend 2 & 60 & TL Neph & TL Neph & TL Neph & TL Neph & TL newlv Neph & TL newlv Neph & TL Nepl \\
\hline 1 & Case 7 Batch 1 & 25 & newhv & newhv & newhy & newhy & & & \\
\hline 1 & Case 7 Batch 1 & 26 & newhv & newhv & newhy & newhy & & & \\
\hline 1 & Case 7 Batch 1 & 27 & newhy & newhv & newhy & newhy & & & \\
\hline 1 & Case 7 Batch 1 & 28 & newhv & newhv & newhy & newhy & & & \\
\hline 1 & Case 7 Batch 1 & 29 & newhv & newhv & newhy & & & & \\
\hline 1 & $\begin{array}{l}\text { Case } 7 \text { Batch } 1 \\
\end{array}$ & 30 & newhv & newhv & newhy & & & & \\
\hline 1 & Case 7 Batch 1 & 31 & newhv & newhv & newhy & & & & \\
\hline 1 & Case 7 Batch 1 & 32 & newhy & newhy & newhy & & & & \\
\hline 1 & Case 7 Batch 1 & 33 & newhv & newhv & newhy & & & & \\
\hline 1 & Case 7 Batch 1 & 34 & newhv & newhv & newhy & & & & \\
\hline 1 & Case 7 Batch 1 & 35 & newhv & newhv & newhy & & & & \\
\hline 1 & Case 7 Batch 1 & 36 & newhy & newhv & newhy & & & & \\
\hline 1 & Case 7 Batch 1 & 37 & newhv & newhv & newhy & & & & \\
\hline 1 & Case 7 Batch 1 & 38 & newhv & newhv & newhy & & & & \\
\hline 1 & Case 7 Batch 1 & 39 & newhy & newhy & newhy & & & & \\
\hline 1 & Case 7 Batch 1 & 40 & newhv & newhv & newhy & & & & \\
\hline 1 & Case 7 Batch 1 & 41 & newhv & newhv & & & & & \\
\hline 1 & Case 7 Batch 1 & 42 & newhy & newhv & & & & Neph & \\
\hline 1 & Case 7 Batch 1 & 43 & newhv & newhv & & $\mathrm{TL}$ & Neph & Neph & \\
\hline 1 & Case 7 Batch 1 & 44 & newhv & newhv & & TL Neph & Neph & Neph & Neph \\
\hline 1 & Case 7 Batch 1 & 45 & newhv & & Neph & TL Neph & TL Neph & TL Neph & Neph \\
\hline 1 & Case 7 Batch 1 & 46 & TL newhv & Neph & Neph & TL Neph & TL Neph & TL Neph & Neph \\
\hline 1 & Case 7 Batch 1 & 47 & TL newhy Neph & TL Neph & Neph & TL Neph & TL Neph & TL newlv Neph & Neph \\
\hline 1 & $\begin{array}{l}\text { Case } 7 \text { Batch } 1 \\
\end{array}$ & 48 & TL newhy Neph & TL Neph & TL Neph & TL Neph & TL Neph & TL newlv Neph & Neph \\
\hline
\end{tabular}


Exhibit A1. Results for MAR Assessments of the Nominal Stage (part 1)

Constraint not met: neph (nepheline), TL (liquidus temperature), newlv (low viscosity - new model), newhv (high viscosity - new model), and Del GP - durability

\begin{tabular}{|c|c|c|c|c|c|c|c|c|c|}
\hline & & $\mathrm{Na} 2 \mathrm{O}$ & 0.03 & 0.04 & 0.05 & 0.05 & 0.05 & 0.05 & 0.05 \\
\hline Set & Type & $\begin{array}{l}\text { Sludge Loading (\%) } \\
\end{array}$ & 422 & $\mathrm{~d} 1-1$ & 473 & P1-1 & P2-1 & P2-2 & $\begin{array}{ll}\text { P3-1 } \\
\end{array}$ \\
\hline 1 & Case 7 Batch 1 & 49 & TL Neph & TL Neph & TL Neph & TL Neph & TL Neph & TL newlv Neph & Neph \\
\hline 1 & Case 7 Batch 1 & 50 & TL Neph & TL Neph & TL Neph & TL Neph & TL Neph & TL newlv Neph & Neph \\
\hline 1 & Case 7 Batch 1 & 51 & TL Neph & TL Neph & TL Neph & TL Neph & TL Neph & TL newlv Neph & TL Neph \\
\hline 1 & Case 7 Batch 1 & 52 & TL Neph & TL Neph & TL Neph & TL Neph & TL Neph & TL newlv Neph & TL Neph \\
\hline 1 & Case 7 Batch 1 & 53 & TL Neph & TL Neph & TL Neph & TL Neph & TL Neph & TL newlv Neph & TL Neph \\
\hline 1 & Case 7 Batch 1 & 54 & TL Neph & TL Neph & TL Neph & TL Neph & TL Neph & TL newlv Neph & TL Neph \\
\hline 1 & Case 7 Batch 1 & 55 & TL Neph & TL Neph & TL Neph & TL Neph & TL Neph & TL newlv Neph & TL Neph \\
\hline 1 & Case 7 Batch 1 & 56 & TL Neph & TL Neph & TL Neph & TL Neph & TL newlv Neph & TL newlv Neph & TL Neph \\
\hline 1 & Case 7 Batch 1 & 57 & TL Neph & TL Neph & TL Neph & TL Neph & TL newlv Neph & TL newlv Neph & TL Neph \\
\hline 1 & Case 7 Batch 1 & 58 & TL Neph & TL Neph & TL Neph & TL Neph & TL newlv Neph & TL newlv Neph & TL newlv Neph \\
\hline 1 & Case 7 Batch 1 & 59 & TL Neph & TL Neph & TL Neph & TL Neph & TL newlv Neph & TL newlv Neph & TL newly Neph \\
\hline 1 & Case 7 Batch 1 & 60 & TL Neph & TL Neph & TL Neph & TL Neph & TL newlv Neph & TL newlv Neph & TL newlv Neph \\
\hline 1 & Case 7 Batch 2 & 25 & newhv & newhv & newhv & newhv & & & \\
\hline 1 & Case 7 Batch 2 & 26 & newhv & newhv & newhy & newhy & & & \\
\hline 1 & Case 7 Batch 2 & 27 & newhv & newhv & newhv & newhv & & & \\
\hline 1 & Case 7 Batch 2 & 28 & newhv & newhv & newhv & newhv & & & \\
\hline 1 & Case 7 Batch 2 & 29 & newhy & newhv & newhy & newhy & & & \\
\hline 1 & Case 7 Batch 2 & 30 & newhv & newhv & newhv & newhv & & & \\
\hline 1 & Case 7 Batch 2 & 31 & newhv & newhv & newhv & newhv & & & \\
\hline 1 & Case 7 Batch 2 & 32 & newhy & newhv & newhy & newhy & & & \\
\hline 1 & Case 7 Batch 2 & 33 & newhy & newhv & newhv & newhv & & & \\
\hline 1 & Case 7 Batch 2 & 34 & newhv & newhv & newhy & newhv & & & \\
\hline 1 & Case 7 Batch 2 & 35 & newhy & newhv & newhy & newhy & & & \\
\hline 1 & Case 7 Batch 2 & 36 & newhv & newhv & newhv & TL newhy & & & \\
\hline 1 & Case 7 Batch 2 & 37 & newhv & newhy & newhv & TL newhv & & & \\
\hline 1 & Case 7 Batch 2 & 38 & TL newhv & newhv & newhv & TL newhv & $\mathrm{TL}$ & $\mathrm{TL}$ & \\
\hline 1 & Case 7 Batch 2 & 39 & TL newhy & newhv & newhv & TL newhy & $\mathrm{TL}$ & $\mathrm{TL}$ & \\
\hline 1 & Case 7 Batch 2 & 40 & TL newhy & TL newhv & newhy & TL newhv & $\mathrm{TL}$ & $\mathrm{TL}$ & \\
\hline 1 & Case 7 Batch 2 & 41 & TL newhy & TL newhv & TL newhv & TL newhv & $\mathrm{TL}$ & $\mathrm{TL}$ & \\
\hline 1 & Case 7 Batch 2 & 42 & TL newhy & TL newhy & TL newhy & TL newhy & $\mathrm{TL}$ & TL & \\
\hline 1 & Case 7 Batch 2 & 43 & TL newhy & TL newhy & TL newhy & TL newhv & $\mathrm{TL}$ & $\mathrm{TL}$ & $\mathrm{TL}$ \\
\hline 1 & Case 7 Batch 2 & 44 & TL newhv & TL newhv & TL newhv & TL newhv & TL Neph & TL Neph & $\mathrm{TL}$ \\
\hline 1 & Case 7 Batch 2 & 45 & TL newhv & TL newhv & TL newhv & TL Neph & TL Neph & TL Neph & TL Neph \\
\hline 1 & Case 7 Batch 2 & 46 & TL newhv & TL newhy & TL newhy & TL Neph & TL Neph & TL Neph & TL Neph \\
\hline 1 & Case 7 Batch 2 & 47 & TL newhy & TL newhy Neph & TL newhy Neph & TL Neph & TL Neph & TL Neph & TL Neph \\
\hline 1 & Case 7 Batch 2 & 48 & TL newhy Neph & TL newhy Neph & TL newhy Neph & TL Neph & TL Neph & TL Neph & TL Neph \\
\hline 1 & Case 7 Batch 2 & 49 & TL newhv Neph & TL newhy Neph & TL newhv Neph & TL Neph & TL Neph & TL Neph & TL Neph \\
\hline 1 & Case 7 Batch 2 & 50 & TL newhy Neph & TL newhy Neph & TL newhy Neph & TL Neph & TL Neph & TL Neph & TL Neph \\
\hline 1 & Case 7 Batch 2 & 51 & TL newhy Neph & TL newhy Neph & TL newhy Neph & TL Neph & TL Neph & TL Neph & TL Neph \\
\hline 1 & Case 7 Batch 2 & 52 & TL newhy Neph & TL newhy Neph & TL newhy Neph & TL Neph & TL Neph & TL Neph & TL Neph \\
\hline 1 & Case 7 Batch 2 & 53 & TL newhy Neph & TL newhy Neph & TL newhy Neph & TL Neph & TL Neph & TL Neph & TL Neph \\
\hline 1 & Case 7 Batch 2 & 54 & TL newhy Neph & TL newhv Neph & TL newhy Neph & TL Neph & TL Neph & TL Neph & TL Neph \\
\hline 1 & Case 7 Batch 2 & 55 & TL newhy Neph & TL newhy Neph & TL newhy Neph & TL Neph & TL Neph & TL Neph & TL Neph \\
\hline 1 & Case 7 Batch 2 & 56 & TL newhy Neph & TL newhy Neph & TL newhv Neph & TL Neph & TL Neph & TL Neph & TL Neph \\
\hline 1 & Case 7 Batch 2 & 57 & TL newhv Neph & TL newhy Neph & TL newhy Neph & TL Neph & TL Neph & TL Neph & TL Neph \\
\hline 1 & Case 7 Batch 2 & 58 & TL newhy Neph & TL newhy Neph & TL newhy Neph & TL Neph & TL Neph & TL Neph & TL Neph \\
\hline 1 & Case 7 Batch 2 & 59 & TL newhy Neph & TL newhy Neph & TL Neph & TL Neph & TL Neph & TL Neph & TL Neph \\
\hline 1 & Case 7 Batch 2 & 60 & TL newhy Neph & TL newhy Neph & TL Neph & TL Neph & TL Neph & TL Neph & TL Neph \\
\hline 1 & Case 7 Blend 1 & 25 & newhv & newhv & newhv & & & & \\
\hline 1 & Case 7 Blend 1 & 26 & newhv & newhv & newhy & & & & \\
\hline 1 & Case 7 Blend 1 & 27 & newhv & newhv & & & & & \\
\hline 1 & Case 7 Blend 1 & 28 & newhy & newhy & & & & & \\
\hline
\end{tabular}


Exhibit A1. Results for MAR Assessments of the Nominal Stage (part 1)

Constraint not met: neph (nepheline), TL (liquidus temperature), newlv (low viscosity - new model), newhv (high viscosity - new model), and Del GP - durability

\begin{tabular}{|c|c|c|c|c|c|c|c|c|c|}
\hline & & Na2O & 0.03 & 0.04 & 0.05 & 0.05 & 0.05 & 0.05 & 0.05 \\
\hline Set & Type & Sludge Loading (\%) & 422 & $\begin{array}{ll}\mathrm{d} 1-1 \\
\end{array}$ & 473 & P1-1 & $\mathrm{P} 2-1$ & P2-2 & P3-1 \\
\hline 1 & Case 7 Blend 1 & 29 & newhv & newhv & & & & & \\
\hline 1 & Case 7 Blend 1 & 30 & newhv & & & & & & \\
\hline 1 & Case 7 Blend 1 & 31 & newhv & & & & & newlv & \\
\hline 1 & Case 7 Blend 1 & 32 & newhy & & & & & newlv & \\
\hline 1 & Case 7 Blend 1 & 33 & & & & & & newlv & \\
\hline 1 & Case 7 Blend 1 & 34 & & & & & & newlv & \\
\hline 1 & Case 7 Blend 1 & 35 & & & & & & newlv & \\
\hline 1 & Case 7 Blend 1 & 36 & & & & & & newlv & \\
\hline 1 & Case 7 Blend 1 & 37 & & & & & & newlv & \\
\hline 1 & Case 7 Blend 1 & 38 & & & & & & newlv & \\
\hline 1 & Case 7 Blend 1 & 39 & & & & & newlv & newlv & \\
\hline 1 & Case 7 Blend 1 & 40 & & & & $\mathrm{TL}$ & newlv & newlv & \\
\hline 1 & Case 7 Blend 1 & 41 & & & & $\mathrm{TL}$ & newlv & newlv & newlv \\
\hline 1 & Case 7 Blend 1 & 42 & $\mathrm{TL}$ & & & $\mathrm{TL}$ & newlv & newly & newlv \\
\hline 1 & Case 7 Blend 1 & 43 & $\mathrm{TL}$ & $\mathrm{TL}$ & & $\mathrm{TL}$ & TL newlv & TL newlv & newlv \\
\hline 1 & Case 7 Blend 1 & 44 & $\mathrm{TL}$ & $\mathrm{TL}$ & $\mathrm{TL}$ & $\mathrm{TL}$ & TL newlv & TL newlv & newlv \\
\hline 1 & Case 7 Blend 1 & 45 & $\mathrm{TL}$ & $\mathrm{TL}$ & $\mathrm{TL}$ & $\mathrm{TL}$ & TL newlv & TL newlv & newlv \\
\hline 1 & Case 7 Blend 1 & 46 & $\mathrm{TL}$ & $\mathrm{TL}$ & $\mathrm{TL}$ & $\mathrm{TL}$ & TL newlv & TL newlv & newlv \\
\hline 1 & Case 7 Blend 1 & 47 & $\mathrm{TL}$ & $\mathrm{TL}$ & $\mathrm{TL}$ & TL newlv & TL newlv & TL newlv & newlv \\
\hline 1 & Case 7 Blend 1 & 48 & $\mathrm{TL}$ & $\mathrm{TL}$ & $\mathrm{TL}$ & TL newlv & TL newlv & TL newlv & TL newlv \\
\hline 1 & Case 7 Blend 1 & 49 & $\mathrm{TL}$ & $\mathrm{TL}$ & $\mathrm{TL}$ & TL newlv & TL newlv & TL newlv & TL newlv \\
\hline 1 & Case 7 Blend 1 & 50 & $\mathrm{TL}$ & $\mathrm{TL}$ & $\mathrm{TL}$ & TL newlv & TL newlv & TL newlv Neph & TL newlv \\
\hline 1 & Case 7 Blend 1 & 51 & $\mathrm{TL}$ & $\mathrm{TL}$ & $\mathrm{TL}$ & TL newlv & TL newlv Neph & TL newlv Neph & TL newlv \\
\hline 1 & Case 7 Blend 1 & 52 & $\mathrm{TL}$ & $\mathrm{TL}$ & TL newlv & TL newlv Neph & TL newlv Neph & TL newlv Neph & TL newlv Neph \\
\hline 1 & Case 7 Blend 1 & 53 & $\mathrm{TL}$ & $\mathrm{TL}$ & TL newlv Neph & TL newlv Neph & TL newlv Neph & TL newlv Neph & TL newlv Neph \\
\hline 1 & Case 7 Blend 1 & 54 & TL & TL newlv Neph & TL newlv Neph & TL newlv Neph & TL newlv Neph & TL newlv Neph & TL newlv Neph \\
\hline 1 & Case 7 Blend 1 & 55 & TL newlv Neph & TL newlv Neph & TL newlv Neph & TL newlv Neph & TL newlv Neph & TL newlv Neph & TL newlv Neph \\
\hline 1 & Case 7 Blend 1 & 56 & TL newlv Neph & TL newlv Neph & TL newlv Neph & TL newlv Neph & TL newlv Neph & TL newlv Neph & TL newlv Neph \\
\hline 1 & Case 7 Blend 1 & 57 & TL newlv Neph & TL newlv Neph & TL newlv Neph & TL newlv Neph & TL newlv Neph & TL newlv Neph & TL newlv Neph \\
\hline 1 & Case 7 Blend 1 & 58 & TL newlv Neph & TL newlv Neph & TL newlv Neph & TL newlv Neph & TL newlv Neph & TL newlv Neph & TL newlv Neph \\
\hline 1 & Case 7 Blend 1 & 59 & TL newlv Neph & TL newlv Neph & TL newlv Neph & TL newlv Neph & TL newlv Neph & TL newlv Neph & TL newlv Neph \\
\hline 1 & Case 7 Blend 1 & 60 & TL newlv Neph & TL newlv Neph & TL newlv Neph & TL newlv Neph & TL newlv Neph & TL newlv Neph & TL newlv Neph \\
\hline 1 & Case 7 Blend 2 & 25 & newhv & newhv & newhy & newhy & & & \\
\hline 1 & $\begin{array}{l}\text { Case } 7 \text { Blend } 2 \\
\end{array}$ & 26 & newhv & newhv & newhv & newhy & & & \\
\hline 1 & Case 7 Blend 2 & 27 & newhv & newhv & newhy & newhy & & & \\
\hline 1 & Case 7 Blend 2 & 28 & newhy & newhy & newhy & newhy & & & \\
\hline 1 & Case 7 Blend 2 & 29 & newhv & newhv & newhy & newhy & & & \\
\hline 1 & Case 7 Blend 2 & 30 & newhy & newhy & newhv & newhy & & & \\
\hline 1 & Case 7 Blend 2 & 31 & newhv & newhv & newhy & newhy & & & \\
\hline 1 & Case 7 Blend 2 & 32 & newhy & newhv & newhy & newhy & & & \\
\hline 1 & Case 7 Blend 2 & 33 & newhv & newhv & newhy & & & & \\
\hline 1 & Case 7 Blend 2 & 34 & newhv & newhv & newhy & & & & \\
\hline 1 & Case 7 Blend 2 & 35 & newhy & newhy & newhy & & & & \\
\hline 1 & Case 7 Blend 2 & 36 & newhv & newhv & newhy & $\mathrm{TL}$ & & & \\
\hline 1 & Case 7 Blend 2 & 37 & newhv & newhv & newhy & $\mathrm{TL}$ & & & \\
\hline 1 & Case 7 Blend 2 & 38 & newhy & newhv & newhy & $\mathrm{TL}$ & & & \\
\hline 1 & Case 7 Blend 2 & 39 & TL newhy & newhy & newhy & $\mathrm{TL}$ & $\mathrm{TL}$ & $\overline{T L}$ & \\
\hline 1 & Case 7 Blend 2 & 40 & TL newhv & TL newhv & newhy & $\mathrm{TL}$ & $\mathrm{TL}$ & $\mathrm{TL}$ & \\
\hline 1 & Case 7 Blend 2 & 41 & TL newhv & TL newhv & TL newhv & $\mathrm{TL}$ & $\mathrm{TL}$ & $\mathrm{TL}$ & \\
\hline 1 & Case 7 Blend 2 & 42 & TL newhy & TL newhy & TL newhy & $\mathrm{TL}$ & $\mathrm{TL}$ & $\mathrm{TL}$ & \\
\hline 1 & Case 7 Blend 2 & 43 & TL newhy & TL newhy & TL newhy & $\mathrm{TL}$ & $\mathrm{TL}$ & $\mathrm{TL}$ & \\
\hline 1 & Case 7 Blend 2 & 44 & TL newhy & TL newhy & TL newhy & $\mathrm{TL}$ & $\mathrm{TL}$ & $\mathrm{TL}$ & $\mathrm{TL}$ \\
\hline
\end{tabular}


Exhibit A1. Results for MAR Assessments of the Nominal Stage (part 1)

Constraint not met: neph (nepheline), TL (liquidus temperature), newlv (low viscosity - new model), newhv (high viscosity - new model), and Del GP - durability

\begin{tabular}{|c|c|c|c|c|c|c|c|c|c|}
\hline & & $\begin{array}{l}\mathrm{Na} 2 \mathrm{O} \\
\end{array}$ & 0.03 & 0.04 & 0.05 & 0.05 & 0.05 & 0.05 & 0.05 \\
\hline Set & Type & Sludge Loading (\%) & 422 & d1-1 & 473 & P1-1 & P2-1 & P2-2 & P3-1 \\
\hline 1 & Case 7 Blend 2 & 45 & TL newhv & TL newhy & TL newhy & $\mathrm{TL}$ & TL Neph & TL Neph & $\mathrm{TL}$ \\
\hline 1 & Case 7 Blend 2 & 46 & TL newhv & TL newhv & $\mathrm{TL}$ & TL Neph & TL Neph & TL Neph & TL Neph \\
\hline$\frac{1}{1}$ & Case 7 Blend 2 & $\frac{70}{47}$ & TL newhy & TL newhy & $\mathrm{TL}$ & TL Neph & TL Neph & TL Neph & TL Neph \\
\hline 1 & Case 7 Blend 2 & 48 & TL newhy & TL newhy Neph & TL Neph & TL Neph & TL Neph & TL Neph & TL Neph \\
\hline 1 & Case 7 Blend 2 & 49 & TL newhv Neph & TL newhy Neph & TL Neph & TL Neph & TL Neph & TL Neph & TL Neph \\
\hline 1 & Case 7 Blend 2 & 50 & TL newhy Neph & TL newhy Neph & TL Neph & TL Neph & TL Neph & TL Neph & TL Neph \\
\hline 1 & Case 7 Blend 2 & 51 & TL newhv Neph & TL Neph & TL Neph & TL Neph & TL Neph & TL Neph & TL Neph \\
\hline 1 & Case 7 Blend 2 & 52 & TL newhv Neph & TL Neph & TL Neph & TL Neph & TL Neph & TL Neph & TL Neph \\
\hline 1 & Case 7 Blend 2 & 53 & TL newhv Neph & TL Neph & TL Neph & TL Neph & TL Neph & TL newlv Neph & TL Neph \\
\hline 1 & Case 7 Blend 2 & 54 & TL Neph & TL Neph & TL Neph & TL Neph & TL Neph & TL newlv Neph & TL Neph \\
\hline$\frac{1}{1}$ & Case 7 Blend 2 & 55 & TL Neph & TL Neph & TL Neph & TL Neph & TL Neph & TL newlv Neph & TL Neph \\
\hline 1 & Case 7 Blend 2 & 56 & TL Neph & TL Neph & TL Neph & TL Neph & TL Neph & TL newlv Neph & TL Neph \\
\hline 1 & Case 7 Blend 2 & 57 & TL Neph & TL Neph & TL Neph & TL Neph & TL Neph & TL newlv Neph & TL Neph \\
\hline 1 & Case 7 Blend 2 & 58 & TL Neph & TL Neph & TL Neph & TL Neph & TL Neph & TL newlv Neph & TL Neph \\
\hline 1 & Case 7 Blend 2 & 59 & TL Neph & TL Neph & TL Neph & TL Neph & TL Neph & TL newlv Neph & TL Neph \\
\hline 1 & Case 7 Blend 2 & 60 & TL Neph & TL Neph & TL Neph & TL Neph & TL Neph & TL newlv Neph & TL Neph \\
\hline 1 & Case 8 - Blend 1 & 25 & newhy & newhy & newhy & & & & \\
\hline$\frac{1}{1}$ & Case 8 - Blend 1 & $\frac{25}{26}$ & newhy & newhy & newhy & & & & \\
\hline 1 & Case 8 - Blend 1 & 27 & newhv & newhv & newhv & & & & \\
\hline 1 & Case 8 - Blend 1 & 28 & newhy & newhy & newhy & & & & \\
\hline 1 & Case 8 - Blend 1 & 29 & newhy & newhv & newhy & & & & \\
\hline 1 & Case 8 - Blend 1 & 30 & newhy & newhy & & & & & \\
\hline 1 & Case 8 - Blend 1 & 31 & newhv & newhy & & & & & \\
\hline 1 & Case 8 - Blend 1 & 32 & newhy & newhy & & & & & \\
\hline 1 & Case 8 - Blend 1 & 33 & newhy & newhy & & & & & \\
\hline 1 & Case 8 - Blend 1 & 34 & newhy & & & & & & \\
\hline 1 & Case 8 - Blend 1 & 35 & newhv & & & & & & \\
\hline 1 & Case 8 - Blend 1 & $\frac{53}{36}$ & newhy & & & & & newlv & \\
\hline 1 & Case 8 - Blend 1 & 37 & & & & & & newlv & \\
\hline 1 & Case 8 - Blend 1 & 38 & & & & & & newlv & \\
\hline 1 & Case 8 - Blend 1 & 39 & & & & $\mathrm{TL}$ & & newlv & \\
\hline 1 & Case 8 - Blend 1 & 40 & & & & $\mathrm{TL}$ & & newlv & \\
\hline 1 & Case 8 - Blend 1 & 41 & $\overline{T L}$ & & & $\mathrm{TL}$ & $\mathrm{TL}$ & TL newlv & \\
\hline 1 & Case 8 - Blend 1 & 42 & $\mathrm{TL}$ & $\mathrm{TL}$ & & $\mathrm{TL}$ & TL & TL newlv & \\
\hline 1 & Case 8 - Blend 1 & 43 & $\mathrm{TL}$ & $\mathrm{TL}$ & $\mathrm{TL}$ & $\mathrm{TL}$ & TL newlv & TL newlv & \\
\hline$\frac{1}{1}$ & Case 8 - Blend 1 & 44 & $\frac{T L}{T L}$ & $\frac{T L}{T L}$ & $\frac{T}{T L}$ & $\frac{T L}{T L}$ & TL newlv & TL newlv & \\
\hline 1 & Case 8 - Blend 1 & 45 & $\mathrm{TL}$ & $\mathrm{TL}$ & $\mathrm{TL}$ & $\mathrm{TL}$ & TL newlv & TL newlv & \\
\hline 1 & Case 8 - Blend 1 & 46 & $\mathrm{TL}$ & $\mathrm{TL}$ & $\mathrm{TL}$ & $\mathrm{TL}$ & TL newlv & TL newlv & TL newlv \\
\hline 1 & Case 8 - Blend 1 & 47 & $\mathrm{TL}$ & $\mathrm{TL}$ & $\mathrm{TL}$ & $\mathrm{TL}$ & TL newlv & TL newlv Neph & TL newlv \\
\hline 1 & Case 8 - Blend 1 & 48 & $\mathrm{TL}$ & $\mathrm{TL}$ & $\mathrm{TL}$ & $\mathrm{TL}$ & TL newlv Neph & TL newlv Neph & TL newlv \\
\hline 1 & Case 8 - Blend 1 & 49 & $\mathrm{TL}$ & $\mathrm{TL}$ & $\mathrm{TL}$ & TL Neph & TL newlv Neph & TL newlv Neph & TL newlv Neph \\
\hline 1 & Case 8 - Blend 1 & 50 & $\mathrm{TL}$ & $\mathrm{TL}$ & TL Neph & TL Neph & TL newlv Neph & TL newlv Neph & TL newlv Neph \\
\hline$\frac{1}{1}$ & Case 8 - Blend 1 & 51 & TL & TL Neph & TL Neph & TL Neph & TL newlv Neph & TL newlv Neph & TL newly Neph \\
\hline 1 & Case 8 - Blend 1 & 52 & TL Neph & TL Neph & TL Neph & TL newlv Neph & TL newlv Neph & TL newlv Neph & TL newlv Neph \\
\hline 1 & Case 8 - Blend 1 & 53 & TL Neph & TL Neph & TL Neph & TL newlv Neph & TL newlv Neph & TL newlv Neph & TL newlv Neph \\
\hline 1 & Case 8 - Blend 1 & 54 & TL Neph & TL Neph & TL Neph & TL newlv Neph & TL newlv Neph & TL newlv Neph & TL newlv Neph \\
\hline 1 & Case 8 - Blend 1 & 55 & TL Neph & TL Neph & TL Neph & TL newlv Neph & TL newlv Neph & TL newlv Neph & TL newlv Neph \\
\hline 1 & Case 8 - Blend 1 & 56 & TL Neph & TL Neph & TL Neph & TL newlv Neph & TL newlv Neph & TL newlv Neph & TL newlv Neph \\
\hline 1 & Case 8 - Blend 1 & 57 & TL Neph & TL Neph & TL newlv Neph & TL newlv Neph & TL newlv Neph & TL newlv Neph & TL newlv Neph \\
\hline 1 & Case 8 - Blend 1 & 58 & TL Neph & TL newlv Neph & TL newlv Neph & TL newlv Neph & TL newlv Neph & TL newlv Neph & TL newly Neph \\
\hline 1 & Case 8 - Blend 1 & 59 & TL Neph & TL newlv Neph & TL newlv Neph & TL newlv Neph & TL newlv Neph & TL newlv Neph & TL newlv Neph \\
\hline 1 & Case 8 - Blend 1 & 60 & TL newlv Neph & TL newlv Neph & TL newlv Neph & TL newlv Neph & TL newlv Neph & TL newlv Neph & TL newlv Neph \\
\hline
\end{tabular}


Exhibit A1. Results for MAR Assessments of the Nominal Stage (part 1)

Constraint not met: neph (nepheline), TL (liquidus temperature), newlv (low viscosity - new model), newhv (high viscosity - new model), and Del GP - durability

\begin{tabular}{|c|c|c|c|c|c|c|c|c|c|}
\hline & & ( $\mathrm{Na} 2 \mathrm{O}$ & 0.03 & 0.04 & 0.05 & 0.05 & 0.05 & 0.05 & 0.05 \\
\hline Set & Type & Sludge Loading (\%) & 422 & $\mathrm{~d} 1-1$ & 473 & P1-1 & P2-1 & P2-2 & P3-1 \\
\hline 1 & Case 8 and $8 \mathrm{a}$ - Batch 1 & 25 & newhy & newhv & newhv & newhy & & & \\
\hline 1 & Case 8 and $8 \mathrm{a}-$ - Batch 1 & 26 & newhy & newhy & newhy & & & & \\
\hline 1 & Case 8 and $8 \mathrm{a}-$ - Batch 1 & 27 & newhy & newhy & newhy & & & & \\
\hline 1 & Case 8 and $8 \mathrm{a}$ - Batch 1 & 28 & newhy & newhv & newhy & & & & \\
\hline 1 & Case 8 and $8 \mathrm{a}-$ - Batch 1 & 29 & newhy & newhy & newhy & & & & \\
\hline 1 & Case 8 and $8 a-$ Batch 1 & 30 & newhy & newhv & newhy & & & & \\
\hline$\frac{1}{1}$ & Case 8 and $8 \mathrm{a}$ - Batch 1 & 31 & newhy & newhy & newhy & & & & \\
\hline 1 & Case 8 and $8 \mathrm{a}-$ Batch 1 & 32 & newhy & newhv & newhy & & & & \\
\hline 1 & Case 8 and $8 \mathrm{a}-$ - Batch 1 & 33 & newhy & newhy & newhy & & & & \\
\hline 1 & Case 8 and $8 \mathrm{a}-$ Batch 1 & 34 & newhv & newhy & newhv & & & & \\
\hline$\frac{1}{1}$ & Case 8 and $8 \mathrm{a}$ - Batch 1 & 35 & newhy & newhy & newhy & & & & \\
\hline 1 & Case 8 and $8 \mathrm{a}-$ Batch 1 & 36 & newhv & newhv & newhv & & & & \\
\hline 1 & Case 8 and $8 a-$ Batch 1 & 37 & newhy & newhv & newhy & & & & \\
\hline 1 & Case 8 and $8 \mathrm{a}-$ - Batch 1 & 38 & newhy & newhy & & $\mathrm{TL}$ & & & \\
\hline$\frac{1}{1}$ & Case 8 and $8 \mathrm{a}-$ Batch 1 & 39 & newhy & newhy & & $\mathrm{TL}$ & & & \\
\hline 1 & Case 8 and $8 \mathrm{a}-$ - Batch 1 & 40 & newhy & newhy & & $\mathrm{TL}$ & $\mathrm{TL}$ & $\mathrm{TL}$ & \\
\hline 1 & Case 8 and $8 a-$ Batch 1 & 41 & TL newhy & newhv & & $\mathrm{TL}$ & $\mathrm{TL}$ & $\mathrm{TL}$ & \\
\hline$\frac{1}{1}$ & Case 8 and $8 \mathrm{a}$ - Batch 1 & $\frac{42}{42}$ & TL newhy & $\mathrm{TL}$ & & $\frac{T L}{T L}$ & $\frac{T L}{T L}$ & $\frac{T L}{T L}$ & \\
\hline 1 & Case 8 and $8 \mathrm{a}$ - Batch 1 & 43 & TL newhy & $\mathrm{TL}$ & $\mathrm{TL}$ & $\mathrm{TL}$ & $\mathrm{TL}$ & $\mathrm{TL}$ & \\
\hline 1 & Case 8 and $8 \mathrm{a}-$ - Batch 1 & 44 & TL newhy & $\mathrm{TL}$ & $\mathrm{TL}$ & $\mathrm{TL}$ & $\mathrm{TL}$ & TL newlv Neph & \\
\hline 1 & Case 8 and $8 \mathrm{a}-$ - Batch 1 & 45 & TL newhy & $\mathrm{TL}$ & $\mathrm{TL}$ & $\mathrm{TL}$ & TL Neph & TL newlv Neph & \\
\hline 1 & Case 8 and $8 \mathrm{a}$ - Batch 1 & 46 & TL & $\mathrm{TL}$ & $\mathrm{TL}$ & TL Neph & TL Neph & TL newlv Neph & TL Neph \\
\hline 1 & Case 8 and $8 \mathrm{a}-$ Batch 1 & 47 & $\mathrm{TL}$ & $\mathrm{TL}$ & TL Neph & TL Neph & TL Neph & TL newlv Neph & TL Neph \\
\hline 1 & Case 8 and $8 \mathrm{a}$ - Batch 1 & 48 & $\mathrm{TL}$ & TL Neph & TL Neph & TL Neph & TL Neph & TL newlv Neph & TL Neph \\
\hline$\frac{1}{1}$ & Case 8 and $8 \mathrm{a}$ - Batch 1 & 49 & TL Neph & TL Neph & TL Neph & TL Neph & TL Neph & TL newlv Neph & TL Neph \\
\hline 1 & Case 8 and $8 \mathrm{a}$ - Batch 1 & 50 & TL Neph & TL Neph & TL Neph & TL Neph & TL Neph & TL newlv Neph & TL Neph \\
\hline 1 & Case 8 and $8 \mathrm{a}-$ Batch 1 & 51 & TL Neph & TL Neph & TL Neph & TL Neph & TL Neph & TL newlv Neph & TL Neph \\
\hline$\frac{1}{1}$ & Case 8 and $8 \mathrm{a}-$ Batch 1 & $\frac{51}{52}$ & TL Neph & TL Neph & TL Neph & TL Neph & TL newlv Neph & TL newlv Neph & TL Neph \\
\hline 1 & Case 8 and $8 \mathrm{a}$ - Batch 1 & 53 & TL Neph & TL Neph & TL Neph & TL Neph & TL newlv Neph & TL newlv Neph & TL Neph \\
\hline 1 & Case 8 and $8 \mathrm{a}-$ Batch 1 & 54 & TL Neph & TL Neph & TL Neph & TL Neph & TL newlv Neph & TL newlv Neph & TL Neph \\
\hline 1 & Case 8 and $8 a-$ Batch 1 & 55 & TL Neph & TL Neph & TL Neph & TL Neph & TL newlv Neph & TL newlv Neph & TL newlv Neph \\
\hline 1 & Case 8 and $8 \mathrm{a}-$ - Batch 1 & 56 & TL Neph & TL Neph & TL Neph & TL Neph & TL newlv Neph & TL newlv Neph & TL newlv Neph \\
\hline 1 & Case 8 and $8 \mathrm{a}-$ - Batch 1 & 57 & TL Neph & TL Neph & TL Neph & TL Neph & TL newlv Neph & TL newlv Neph & TL newlv Neph \\
\hline 1 & Case 8 and $8 \mathrm{a}-$ Batch 1 & 58 & TL Neph & TL Neph & TL Neph & TL Neph & TL newlv Neph & TL newlv Neph & TL newlv Neph \\
\hline 1 & Case 8 and $8 a-$ Batch 1 & 59 & TL Neph & TL Neph & TL Neph & TL Neph & TL newlv Neph & TL newlv Neph & TL newlv Neph \\
\hline$\frac{1}{1}$ & Case 8 and $8 \mathrm{a}$ - Batch 1 & 60 & TL Neph & TL Neph & TL Neph & TL Neph & TL newlv Neph & TL newlv Neph & TL newlv Neph \\
\hline 1 & Case $8 \mathrm{~A}$ - Blend 1 & 25 & newhy & newhy & newhy & & & & \\
\hline 1 & Case 8A - Blend 1 & 26 & newhy & newhy & newhy & & & & \\
\hline 1 & Case $8 \mathrm{~A}$ - Blend 1 & 27 & newhy & newhy & newhy & & & & \\
\hline 1 & Case 8A - Blend 1 & 28 & newhy & newhy & newhy & & & & \\
\hline 1 & Case 8A - Blend 1 & 29 & newhv & newhy & newhy & & & & \\
\hline 1 & Case $8 \mathrm{~A}$ - Blend 1 & 30 & newhv & newhv & newhy & & & & \\
\hline$\frac{1}{1}$ & Case 8A - Blend 1 & 31 & newhy & newhv & newhy & & & & \\
\hline 1 & Case 8A - Blend 1 & 32 & newhv & newhv & newhv & & & & \\
\hline 1 & Case 8A - Blend 1 & 33 & newhv & newhv & & & & & \\
\hline 1 & Case 8A - Blend 1 & 34 & newhv & newhv & & & & & \\
\hline 1 & Case $8 \mathrm{~A}$ - Blend 1 & 35 & newhv & newhv & & & & & \\
\hline 1 & Case 8A - Blend 1 & 36 & newhv & newhv & & & & & \\
\hline 1 & Case $8 \mathrm{~A}$ - Blend 1 & 37 & newhy & & & & & & \\
\hline 1 & Case $8 \mathrm{~A}$ - Blend 1 & 38 & newhy & & & & & & \\
\hline 1 & Case 8A - Blend 1 & 39 & newhy & & & $\mathrm{TL}$ & & newlv & \\
\hline 1 & Case 8A - Blend 1 & 40 & newhy & & & TL & & newlv & \\
\hline
\end{tabular}


Exhibit A1. Results for MAR Assessments of the Nominal Stage (part 1)

Constraint not met: neph (nepheline), TL (liquidus temperature), newlv (low viscosity - new model), newhv (high viscosity - new model), and Del GP - durability

\begin{tabular}{|c|c|c|c|c|c|c|c|c|c|}
\hline & & $\mathrm{Na} 2 \mathrm{O}$ & 0.03 & 0.04 & 0.05 & 0.05 & 0.05 & 0.05 & 0.05 \\
\hline Set & Type & $\begin{array}{l}\text { Sludge Loading }(\%) \\
\end{array}$ & 422 & d1-1 & 473 & P1-1 & P2-1 & P2-2 & P3-1 \\
\hline 1 & Case $8 \mathrm{~A}$ - Blend 1 & 41 & $\mathrm{TL}$ & & & $\mathrm{TL}$ & $\mathrm{TL}$ & TL newlv & \\
\hline 1 & Case $8 \mathrm{~A}$ - Blend 1 & 42 & $\mathrm{TL}$ & $\mathrm{TL}$ & & $\mathrm{TL}$ & $\mathrm{TL}$ & TL newlv & \\
\hline 1 & Case $8 \mathrm{~A}$ - Blend 1 & 43 & $\mathrm{TL}$ & $\mathrm{TL}$ & $\mathrm{TL}$ & $\mathrm{TL}$ & $\mathrm{TL}$ & TL newlv & \\
\hline 1 & $\begin{array}{l}\text { Case } 8 \mathrm{~A} \text { - Blend } 1 \\
\end{array}$ & 44 & $\mathrm{TL}$ & $\mathrm{TL}$ & $\mathrm{TL}$ & $\mathrm{TL}$ & $\mathrm{TL}$ & TL newlv & \\
\hline 1 & Case $8 \mathrm{~A}$ - Blend 1 & 45 & $\mathrm{TL}$ & $\mathrm{TL}$ & $\mathrm{TL}$ & $\mathrm{TL}$ & $\mathrm{TL}$ & TL newlv & \\
\hline 1 & Case $8 \mathrm{~A}$ - Blend 1 & 46 & $\mathrm{TL}$ & $\mathrm{TL}$ & $\mathrm{TL}$ & $\mathrm{TL}$ & TL Neph & TL newlv Neph & $\mathrm{TL}$ \\
\hline 1 & $\begin{array}{l}\text { Case } 8 \mathrm{~A} \text { - Blend } 1 \\
\end{array}$ & 47 & $\mathrm{TL}$ & $\mathrm{TL}$ & $\mathrm{TL}$ & TL Neph & TL newlv Neph & TL newlv Neph & TL Neph \\
\hline 1 & Case $8 \mathrm{~A}$ - Blend 1 & 48 & $\mathrm{TL}$ & $\mathrm{TL}$ & $\mathrm{TL}$ & TL Neph & TL newlv Neph & TL newlv Neph & TL Neph \\
\hline 1 & Case $8 \mathrm{~A}$ - Blend 1 & 49 & $\mathrm{TL}$ & TL Neph & TL Neph & TL Neph & TL newlv Neph & TL newlv Neph & TL newlv Neph \\
\hline 1 & Case 8A-Blend 1 & 50 & TL Neph & TL Neph & TL Neph & TL Neph & TL newlv Neph & TL newlv Neph & TL newlv Neph \\
\hline 1 & Case $8 \mathrm{~A}$ - Blend 1 & 51 & TL Neph & TL Neph & TL Neph & TL Neph & TL newlv Neph & TL newlv Neph & TL newlv Neph \\
\hline 1 & Case $8 \mathrm{~A}$ - Blend 1 & 52 & TL Neph & TL Neph & TL Neph & TL Neph & TL newlv Neph & TL newlv Neph & TL newlv Neph \\
\hline 1 & Case $8 \mathrm{~A}$ - Blend 1 & 53 & TL Neph & TL Neph & TL Neph & TL Neph & TL newlv Neph & TL newlv Neph & TL newlv Neph \\
\hline 1 & Case $8 \mathrm{~A}$ - Blend 1 & 54 & TL Neph & TL Neph & TL Neph & TL Neph & TL newlv Neph & TL newlv Neph & TL newlv Neph \\
\hline 1 & Case $8 \mathrm{~A}$ - Blend 1 & 55 & TL Neph & TL Neph & TL Neph & TL newlv Neph & TL newlv Neph & TL newlv Neph & TL newlv Neph \\
\hline 1 & Case $8 \mathrm{~A}$ - Blend 1 & 56 & TL Neph & TL Neph & TL Neph & TL newlv Neph & TL newlv Neph & TL newlv Neph & TL newlv Neph \\
\hline 1 & Case $8 \mathrm{~A}$ - Blend 1 & 57 & TL Neph & TL Neph & TL Neph & TL newlv Neph & TL newlv Neph & TL newlv Neph & TL newlv Neph \\
\hline 1 & Case $8 \mathrm{~A}$ - Blend 1 & 58 & TL Neph & TL Neph & TL Neph & TL newlv Neph & TL newlv Neph & TL newlv Neph & TL newlv Neph \\
\hline 1 & Case $8 \mathrm{~A}$ - Blend 1 & 59 & TL Neph & TL Neph & TL Neph & TL newlv Neph & TL newlv Neph & TL newlv Neph & TL newlv Neph \\
\hline 1 & Case 8A - Blend 1 & 60 & TL Neph & TL Neph & TL newlv Neph & TL newlv Neph & TL newlv Neph & TL newlv Neph & TL newlv Neph \\
\hline 2 & Case 15 Batch 1 & 25 & newhy & newhy & newhv & newhv & newhv & & newhy \\
\hline 2 & Case 15 Batch 1 & 26 & newhv & newhy & newhy & newhv & newhv & & newhy \\
\hline 2 & Case 15 Batch 1 & 27 & newhv & newhv & newhy & newhy & newhv & & newhy \\
\hline 2 & Case 15 Batch 1 & 28 & newhy & newhy & newhy & newhy & newhv & & newhy \\
\hline 2 & Case 15 Batch 1 & 29 & newhv & newhv & newhy & newhy & newhv & & newhy \\
\hline 2 & Case 15 Batch 1 & 30 & newhv & newhv & newhv & newhy & newhv & & newhv \\
\hline 2 & Case 15 Batch 1 & 31 & newhv & newhv & newhy & newhy & newhv & & newhy \\
\hline 2 & Case 15 Batch 1 & 32 & newhy & newhy & newhy & newhy & newhv & & newhy \\
\hline 2 & Case 15 Batch 1 & 33 & newhv & newhv & newhy & newhv & newhv & & newhy \\
\hline 2 & Case 15 Batch 1 & 34 & newhy & newhv & newhy & newhy & newhv & & newhy \\
\hline 2 & Case 15 Batch 1 & 35 & newhy & newhy & newhy & newhv & newhv & & newhy \\
\hline 2 & $\begin{array}{l}\text { Case } 15 \text { Batch } 1 \\
\end{array}$ & 36 & newhv & newhv & newhy & newhy & newhv & & newhy \\
\hline 2 & Case 15 Batch 1 & 37 & newhv & newhv & newhv & newhy & newhv & & newhy \\
\hline 2 & Case 15 Batch 1 & 38 & newhv & newhv & newhy & newhy & newhv & & newhv \\
\hline 2 & Case 15 Batch 1 & 39 & newhy & newhy & newhy & newhy & newhv & & newhy \\
\hline 2 & $\begin{array}{l}\text { Case } 15 \text { Batch } 1 \\
\end{array}$ & 40 & newhv & newhv & newhy & newhy & newhv & Neph & newhy \\
\hline 2 & Case 15 Batch 1 & 41 & newhy & newhv & newhy & newhv & newhv Neph & Neph & newhv \\
\hline 2 & Case 15 Batch 1 & 42 & newhv & newhv & newhy & newhv Neph & newhv Neph & Neph & newhv Neph \\
\hline 2 & Case 15 Batch 1 & 43 & newhy & newhy & newhv Neph & TL newhy Neph & newhv Neph & Neph & newhv Neph \\
\hline 2 & Case 15 Batch 1 & 44 & newhy & newhv Neph & newhv Neph & TL newhy Neph & newhv Neph & TL Neph & newhv Neph \\
\hline 2 & Case 15 Batch 1 & 45 & newhv Neph & newhv Neph & newhv Neph & TL newhv Neph & TL newhv Neph & TL Neph & newhv Neph \\
\hline 2 & Case 15 Batch 1 & 46 & newhv Neph & newhv Neph & newhv Neph & TL newhv Neph & TL newhy Neph & TL newhv Neph & newhv Neph \\
\hline 2 & Case 15 Batch 1 & 47 & TL newhy Neph & newhv Neph & newhv Neph & TL newhy Neph & TL newhy Neph & TL newhy Neph & newhv Neph \\
\hline 2 & Case 15 Batch 1 & 48 & TL newhy Neph & TL newhy Neph & newhv Neph & TL newhy Neph & TL newhy Neph & TL newhv Neph & newhv Neph \\
\hline 2 & Case 15 Batch 1 & 49 & TL newhy Neph & TL newhy Neph & TL newhy Neph & TL newhy Neph & TL newhy Neph & TL newhv Neph & newhv Neph \\
\hline 2 & Case 15 Batch 1 & 50 & TL newhy Neph & TL newhy Neph & TL newhy Neph & TL newhy Neph & TL newhy Neph & TL newhv Neph & newhv Neph \\
\hline 2 & Case 15 Batch 1 & 51 & TL newhy Neph & TL newhy Neph & TL newhy Neph & TL newhy Neph & TL newhy Neph & TL newhv Neph & TL newhy Neph \\
\hline 2 & $\begin{array}{l}\text { Case } 15 \text { Batch } 1 \\
\end{array}$ & 52 & TL newhy Neph & TL newhy Neph & TL newhy Neph & TL newhy Neph & TL newhy Neph & TL newhv Neph & TL newhv Neph \\
\hline 2 & Case 15 Batch 1 & 53 & TL newhy Neph & TL newhy Neph & TL newhy Neph & TL newhy Neph & TL newhy Neph & TL newhv Neph & TL newhv Neph \\
\hline 2 & $\begin{array}{l}\text { Case } 15 \text { Batch } 1 \\
\end{array}$ & 54 & TL newhy Neph & TL newhy Neph & TL newhy Neph & TL newhy Neph & TL newhy Neph & TL newhv Neph & TL newhv Neph \\
\hline 2 & Case 15 Batch 1 & 55 & TL newhy Neph & TL newhv Neph & TL newhy Neph & TL newhy Neph & TL newhy Neph & TL newhy Neph & TL newhv Neph \\
\hline 2 & Case 15 Batch 1 & 56 & TL newhv Neph & TL newhy Neph & TL newhv Neph & TL newhv Neph & TL newhv Neph & TL newhv Neph & TL newhv Neph \\
\hline
\end{tabular}


Exhibit A1. Results for MAR Assessments of the Nominal Stage (part 1)

Constraint not met: neph (nepheline), TL (liquidus temperature), newlv (low viscosity - new model), newhv (high viscosity - new model), and Del GP - durability

\begin{tabular}{|c|c|c|c|c|c|c|c|c|c|}
\hline & & $\mathrm{Na} 2 \mathrm{O}$ & 0.03 & 0.04 & 0.05 & 0.05 & 0.05 & 0.05 & 0.05 \\
\hline Set & Type & Sludge Loading (\%) & 422 & $\mathrm{~d} 1-1$ & 473 & P1-1 & P2-1 & P2-2 & P3-1 \\
\hline 2 & Case 15 Batch 1 & 57 & TL newhy Neph & TL newhy Neph & TL newhy Neph & TL newhy Neph & TL newhy Neph & TL newhy Neph & TL newhy Neph \\
\hline 2 & Case 15 Batch 1 & 58 & TL newhy Neph & TL newhy Neph & TL newhy Neph & TL newhy Neph & TL newhy Neph & TL newhy Neph & TL newhy Neph \\
\hline 2 & Case 15 Batch 1 & 59 & TL newhy Neph & TL newhy Neph & TL newhv Neph & TL newhy Neph & TL newhy Neph & TL newhy Neph & TL newhy Neph \\
\hline 2 & Case 15 Batch 1 & 60 & TL newhy Neph & TL newhy Neph & TL newhy Neph & TL newhy Neph & TL newhy Neph & TL newhy Neph & TL newhv Neph \\
\hline 2 & Case 15 Batch 2 & 25 & newhy & newhv & newhv & & & & \\
\hline 2 & Case 15 Batch 2 & 26 & newhy & newhy & newhy & & & & \\
\hline 2 & Case 15 Batch 2 & 27 & newhv & newhv & newhy & & & & \\
\hline 2 & Case 15 Batch 2 & 28 & newhy & newhy & newhy & & & & \\
\hline 2 & Case 15 Batch 2 & 29 & newhv & newhv & newhv & & & & \\
\hline 2 & Case 15 Batch 2 & 30 & newhv & newhv & newhy & & & & \\
\hline 2 & Case 15 Batch 2 & 31 & newhy & newhy & newhy & & & & \\
\hline 2 & Case 15 Batch 2 & 32 & newhv & newhv & newhy & & & & \\
\hline 2 & Case 15 Batch 2 & 33 & newhv & newhv & newhy & & & & \\
\hline 2 & Case 15 Batch 2 & 34 & newhv & newhv & newhy & & & & \\
\hline 2 & Case 15 Batch 2 & 35 & newhy & newhy & newhy & & & & \\
\hline 2 & Case 15 Batch 2 & 36 & newhv & newhv & & & & & \\
\hline 2 & Case 15 Batch 2 & 37 & newhv & newhv & & & & & \\
\hline 2 & Case 15 Batch 2 & 38 & newhy & newhy & & & & & \\
\hline 2 & Case 15 Batch 2 & 39 & newhy & newhy & & $\mathrm{TL}$ & & & \\
\hline 2 & Case 15 Batch 2 & 40 & newhy & & & $\mathrm{TL}$ & & & \\
\hline 2 & Case 15 Batch 2 & 41 & newhy & & & $\mathrm{TL}$ & & & \\
\hline 2 & Case 15 Batch 2 & 42 & TL newhy & & & $\mathrm{TL}$ & $\mathrm{TL}$ & TL newlv & \\
\hline 2 & Case 15 Batch 2 & 43 & $\mathrm{TL}$ & $\mathrm{TL}$ & & $\mathrm{TL}$ & $\mathrm{TL}$ & TL newlv & \\
\hline 2 & Case 15 Batch 2 & 44 & $\mathrm{TL}$ & $\mathrm{TL}$ & $\mathrm{TL}$ & $\mathrm{TL}$ & TL Neph & TL newlv Neph & \\
\hline 2 & Case 15 Batch 2 & 45 & $\mathrm{TL}$ & $\mathrm{TL}$ & $\mathrm{TL}$ & TL Neph & TL Neph & TL newlv Neph & \\
\hline 2 & Case 15 Batch 2 & 46 & $\mathrm{TL}$ & $\mathrm{TL}$ & $\mathrm{TL}$ & TL Neph & TL Neph & TL newlv Neph & Neph \\
\hline 2 & Case 15 Batch 2 & 47 & TL & TL & TL Neph & TL Neph & TL Neph & TL newlv Neph & TL Neph \\
\hline 2 & Case 15 Batch 2 & 48 & TL Neph & TL Neph & TL Neph & TL Neph & TL Neph & TL newlv Neph & TL Neph \\
\hline 2 & Case 15 Batch 2 & 49 & TL Neph & TL Neph & TL Neph & TL Neph & TL Neph & TL newlv Neph & TL Neph \\
\hline 2 & Case 15 Batch 2 & 50 & TL Neph & TL Neph & TL Neph & TL Neph & TL newlv Neph & TL newlv Neph & TL Neph \\
\hline 2 & Case 15 Batch 2 & 51 & TL Neph & TL Neph & TL Neph & TL Neph & TL newlv Neph & TL newlv Neph & TL Neph \\
\hline 2 & Case 15 Batch 2 & 52 & TL Neph & TL Neph & TL Neph & TL Neph & TL newlv Neph & TL newlv Neph & TL newlv Neph \\
\hline 2 & Case 15 Batch 2 & 53 & TL Neph & TL Neph & TL Neph & TL Neph & TL newlv Neph & TL newlv Neph & TL newlv Neph \\
\hline 2 & Case 15 Batch 2 & 54 & TL Neph & TL Neph & TL Neph & TL Neph & TL newlv Neph & TL newlv Neph & TL newlv Neph \\
\hline 2 & Case 15 Batch 2 & 55 & TL Neph & TL Neph & TL Neph & TL Neph & TL newlv Neph & TL newlv Neph & TL newlv Neph \\
\hline 2 & Case 15 Batch 2 & 56 & TL Neph & TL Neph & TL Neph & TL Neph & TL newlv Neph & TL newlv Neph & TL newlv Neph \\
\hline 2 & Case 15 Batch 2 & 57 & TL Neph & TL Neph & TL Neph & TL Neph & TL newlv Neph & TL newlv Neph & TL newlv Neph \\
\hline 2 & Case 15 Batch 2 & 58 & TL Neph & TL Neph & TL Neph & TL newlv Neph & TL newlv Neph & TL newlv Neph & TL newlv Neph \\
\hline 2 & Case 15 Batch 2 & 59 & TL Neph & TL Neph & TL Neph & TL newlv Neph & TL newlv Neph & TL newlv Neph & TL newlv Neph \\
\hline 2 & Case 15 Batch 2 & 60 & TL Neph & TL Neph & TL Neph & TL newlv Neph & TL newly Neph & TL newlv Neph & TL newlv Neph \\
\hline 2 & Case 15 Blend 1 & 25 & newhy & newhy & newhv & & & & \\
\hline 2 & Case 15 Blend 1 & 26 & newhv & newhy & newhv & & & & \\
\hline 2 & Case 15 Blend 1 & 27 & newhy & newhy & newhy & & & & \\
\hline 2 & Case 15 Blend 1 & 28 & newhv & newhv & newhy & & & & \\
\hline 2 & Case 15 Blend 1 & 29 & newhv & newhv & newhy & & & & \\
\hline 2 & Case 15 Blend 1 & 30 & newhv & newhv & newhv & & & & \\
\hline 2 & Case 15 Blend 1 & 31 & newhv & newhv & newhy & & & & \\
\hline 2 & Case 15 Blend 1 & 32 & newhv & newhv & newhy & & & & \\
\hline 2 & Case 15 Blend 1 & 33 & newhv & newhv & newhv & & & & \\
\hline 2 & Case 15 Blend 1 & 34 & newhv & newhv & newhy & & & & \\
\hline 2 & Case 15 Blend 1 & 35 & newhv & newhv & & & & & \\
\hline 2 & Case 15 Blend 1 & 36 & newhy & newhy & & & & & \\
\hline
\end{tabular}


Exhibit A1. Results for MAR Assessments of the Nominal Stage (part 1)

Constraint not met: neph (nepheline), TL (liquidus temperature), newlv (low viscosity - new model), newhv (high viscosity - new model), and Del GP - durability

\begin{tabular}{|c|c|c|c|c|c|c|c|c|c|}
\hline & & Na2O & 0.03 & 0.04 & 0.05 & 0.05 & 0.05 & 0.05 & 0.05 \\
\hline Set & Type & Sludge Loading (\%) & 422 & $\begin{array}{ll}\mathrm{d} 1-1 \\
\end{array}$ & 473 & P1-1 & $\mathrm{P} 2-1$ & P2-2 & P3-1 \\
\hline 2 & Case 15 Blend 1 & 37 & newhv & newhv & & & & & \\
\hline 2 & Case 15 Blend 1 & 38 & newhy & newhy & & $\mathrm{TL}$ & & & \\
\hline 2 & Case 15 Blend 1 & 39 & newhv & & & $\mathrm{TL}$ & & & \\
\hline 2 & Case 15 Blend 1 & 40 & newhy & & & $\mathrm{TL}$ & & & \\
\hline 2 & Case 15 Blend 1 & 41 & TL newhv & & & $\mathrm{TL}$ & $\mathrm{TL}$ & TL newlv & \\
\hline 2 & Case 15 Blend 1 & 42 & TL newhy & $\mathrm{TL}$ & & $\mathrm{TL}$ & $\mathrm{TL}$ & TL newlv & \\
\hline 2 & Case 15 Blend 1 & 43 & $\mathrm{TL}$ & $\mathrm{TL}$ & $\mathrm{TL}$ & $\mathrm{TL}$ & $\mathrm{TL}$ & TL newlv & \\
\hline 2 & Case 15 Blend 1 & 44 & $\mathrm{TL}$ & $\mathrm{TL}$ & $\mathrm{TL}$ & $\mathrm{TL}$ & $\mathrm{TL}$ & TL newlv & \\
\hline 2 & Case 15 Blend 1 & 45 & $\mathrm{TL}$ & $\mathrm{TL}$ & $\mathrm{TL}$ & $\mathrm{TL}$ & $\mathrm{TL}$ & TL newlv & \\
\hline 2 & Case 15 Blend 1 & 46 & $\mathrm{TL}$ & $\mathrm{TL}$ & $\mathrm{TL}$ & $\mathrm{TL}$ & $\mathrm{TL}$ & TL newlv & $\mathrm{TL}$ \\
\hline 2 & Case 15 Blend 1 & 47 & $\mathrm{TL}$ & $\mathrm{TL}$ & $\mathrm{TL}$ & $\mathrm{TL}$ & $\mathrm{TL}$ & TL newlv & $\mathrm{TL}$ \\
\hline 2 & Case 15 Blend 1 & 48 & $\mathrm{TL}$ & $\mathrm{TL}$ & $\mathrm{TL}$ & $\mathrm{TL}$ & TL Neph & TL newlv Neph & $\mathrm{TL}$ \\
\hline 2 & Case 15 Blend 1 & 49 & $\mathrm{TL}$ & $\mathrm{TL}$ & $\mathrm{TL}$ & TL Neph & TL newlv Neph & TL newlv Neph & $\mathrm{TL}$ \\
\hline 2 & Case 15 Blend 1 & 50 & $\mathrm{TL}$ & $\mathrm{TL}$ & $\mathrm{TL}$ & TL Neph & TL newlv Neph & TL newlv Neph & TL Neph \\
\hline 2 & Case 15 Blend 1 & 51 & $\mathrm{TL}$ & TL Neph & TL Neph & TL Neph & TL newlv Neph & TL newlv Neph & TL newlv Neph \\
\hline 2 & Case 15 Blend 1 & 52 & TL Neph & TL Neph & TL Neph & TL Neph & TL newlv Neph & TL newlv Neph & TL newlv Neph \\
\hline 2 & Case 15 Blend 1 & 53 & TL Neph & TL Neph & TL Neph & TL Neph & TL newlv Neph & TL newlv Neph & TL newlv Neph \\
\hline 2 & Case 15 Blend 1 & 54 & TL Neph & TL Neph & TL Neph & TL Neph & TL newlv Neph & TL newlv Neph & TL newlv Neph \\
\hline 2 & Case 15 Blend 1 & 55 & TL Neph & TL Neph & TL Neph & TL Neph & TL newlv Neph & TL newlv Neph & TL newlv Neph \\
\hline 2 & Case 15 Blend 1 & 56 & TL Neph & TL Neph & TL Neph & TL Neph & TL newlv Neph & TL newlv Neph & TL newlv Neph \\
\hline 2 & Case 15 Blend 1 & 57 & TL Neph & TL Neph & TL Neph & TL Neph & TL newlv Neph & TL newlv Neph & TL newlv Neph \\
\hline 2 & Case 15 Blend 1 & 58 & TL Neph & TL Neph & TL Neph & TL newlv Neph & TL newlv Neph & TL newlv Neph & TL newlv Neph \\
\hline 2 & Case 15 Blend 1 & 59 & TL Neph & TL Neph & TL Neph & TL newlv Neph & TL newlv Neph & TL newlv Neph & TL newlv Neph \\
\hline 2 & Case 15 Blend 1 & 60 & TL Neph & TL Neph & TL Neph & TL newlv Neph & TL newlv Neph & TL newlv Neph & TL newlv Neph \\
\hline 2 & Case 15 Blend 2 & 25 & newhy & newhv & newhy & & & & \\
\hline 2 & Case 15 Blend 2 & 26 & newhv & newhy & newhy & & & & \\
\hline 2 & Case 15 Blend 2 & 27 & newhy & newhy & newhy & & & & \\
\hline 2 & Case 15 Blend 2 & 28 & newhv & newhv & newhy & & & & \\
\hline 2 & Case 15 Blend 2 & 29 & newhv & newhy & newhy & & & & \\
\hline 2 & Case 15 Blend 2 & 30 & newhv & newhv & newhy & & & & \\
\hline 2 & Case 15 Blend 2 & 31 & newhv & newhv & newhy & & & & \\
\hline 2 & Case 15 Blend 2 & 32 & newhv & newhv & newhy & & & & \\
\hline 2 & Case 15 Blend 2 & 33 & newhv & newhv & newhy & & & & \\
\hline 2 & Case 15 Blend 2 & 34 & newhy & newhy & newhy & & & & \\
\hline 2 & Case 15 Blend 2 & 35 & newhv & newhy & & & & & \\
\hline 2 & Case 15 Blend 2 & 36 & newhy & newhy & & & & & \\
\hline 2 & Case 15 Blend 2 & 37 & newhv & newhy & & & & & \\
\hline 2 & Case 15 Blend 2 & 38 & newhv & newhv & & & & & \\
\hline 2 & Case 15 Blend 2 & 39 & newhy & & & & & & \\
\hline 2 & Case 15 Blend 2 & 40 & newhy & & & $\mathrm{TL}$ & & & \\
\hline 2 & Case 15 Blend 2 & 41 & newhy & & & $\mathrm{TL}$ & & newlv & \\
\hline 2 & Case 15 Blend 2 & 42 & TL newhy & & & $\mathrm{TL}$ & TL & TL newlv & \\
\hline 2 & Case 15 Blend 2 & 43 & $\mathrm{TL}$ & & & $\mathrm{TL}$ & $\mathrm{TL}$ & TL newlv & \\
\hline 2 & Case 15 Blend 2 & 44 & $\mathrm{TL}$ & TL & & $\mathrm{TL}$ & $\mathrm{TL}$ & TL newlv Neph & \\
\hline 2 & Case 15 Blend 2 & 45 & $\mathrm{TL}$ & $\mathrm{TL}$ & $\mathrm{TL}$ & $\mathrm{TL}$ & TL Neph & TL newlv Neph & \\
\hline 2 & Case 15 Blend 2 & 46 & $\mathrm{TL}$ & $\mathrm{TL}$ & $\mathrm{TL}$ & TL Neph & TL Neph & TL newlv Neph & Neph \\
\hline 2 & Case 15 Blend 2 & 47 & $\mathrm{TL}$ & $\mathrm{TL}$ & TL Neph & TL Neph & TL Neph & TL newlv Neph & Neph \\
\hline 2 & Case 15 Blend 2 & 48 & $\mathrm{TL}$ & TL Neph & TL Neph & TL Neph & TL Neph & TL newlv Neph & TL Neph \\
\hline 2 & Case 15 Blend 2 & 49 & TL Neph & TL Neph & TL Neph & TL Neph & TL newlv Neph & TL newlv Neph & TL Neph \\
\hline 2 & Case 15 Blend 2 & 50 & TL Neph & TL Neph & TL Neph & TL Neph & TL newlv Neph & TL newlv Neph & TL Neph \\
\hline 2 & Case 15 Blend 2 & 51 & TL Neph & TL Neph & TL Neph & TL Neph & TL newlv Neph & TL newlv Neph & TL newlv Neph \\
\hline 2 & Case 15 Blend 2 & 52 & TL Neph & TL Neph & TL Neph & TL Neph & TL newlv Neph & TL newlv Neph & TL newlv Neph \\
\hline
\end{tabular}


Exhibit A1. Results for MAR Assessments of the Nominal Stage (part 1)

Constraint not met: neph (nepheline), TL (liquidus temperature), newlv (low viscosity - new model), newhv (high viscosity - new model), and Del GP - durability

\begin{tabular}{|c|c|c|c|c|c|c|c|c|c|}
\hline & & $\mathrm{Na} 2 \mathrm{O}$ & 0.03 & 0.04 & 0.05 & 0.05 & 0.05 & 0.05 & 0.05 \\
\hline Set & Type & $\begin{array}{l}\text { Sludge Loading }(\%) \\
\end{array}$ & 422 & $\mathrm{~d} 1-1$ & 473 & P1-1 & P2-1 & P2-2 & P3-1 \\
\hline 22 & Case 15 Blend 2 & 53 & TL Neph & TL Neph & TL Neph & TL Neph & TL newlv Neph & TL newlv Neph & TL newlv Neph \\
\hline 2 & Case 15 Blend 2 & 54 & TL Neph & TL Neph & TL Neph & TL Neph & TL newlv Neph & TL newlv Neph & TL newlv Neph \\
\hline 2 & Case 15 Blend 2 & 55 & TL Neph & TL Neph & TL Neph & TL Neph & TL newlv Neph & TL newlv Neph & TL newlv Neph \\
\hline 2 & Case 15 Blend 2 & 56 & TL Neph & TL Neph & TL Neph & TL Neph & TL newlv Neph & TL newlv Neph & TL newlv Neph \\
\hline 2 & Case 15 Blend 2 & 57 & TL Neph & TL Neph & TL Neph & TL newlv Neph & TL newlv Neph & TL newlv Neph & TL newlv Neph \\
\hline 2 & Case 15 Blend 2 & 58 & TL Neph & TL Neph & TL Neph & TL newlv Neph & TL newlv Neph & TL newlv Neph & TL newlv Neph \\
\hline 2 & Case 15 Blend 2 & 59 & TL Neph & TL Neph & TL Neph & TL newlv Neph & TL newlv Neph & TL newlv Neph & TL newlv Neph \\
\hline 2 & Case 15 Blend 2 & 60 & TL Neph & TL Neph & TL Neph & TL newlv Neph & TL newlv Neph & TL newlv Neph & TL newlv Neph \\
\hline 3 & Case 7b Batch 1 & 25 & newhy & newhv & newhy & & & & \\
\hline 3 & Case 7b Batch 1 & 26 & newhv & newhy & newhy & & & & \\
\hline 3 & Case 7b Batch 1 & 27 & newhv & newhv & newhy & & & & \\
\hline 3 & Case 7b Batch 1 & 28 & newhv & newhv & newhv & & & & \\
\hline 3 & Case 7b Batch 1 & 29 & newhy & newhy & newhy & & & & \\
\hline 3 & Case 7b Batch 1 & 30 & newhv & newhv & newhy & & & & \\
\hline 3 & Case 7b Batch 1 & 31 & newhv & newhv & newhv & & & & \\
\hline 3 & Case $7 \mathrm{~b}$ Batch 1 & 32 & newhv & newhv & newhy & & & & \\
\hline 3 & Case $7 \mathrm{~b}$ Batch 1 & 33 & newhy & newhy & newhy & & & & \\
\hline 3 & Case 7b Batch 1 & 34 & newhv & newhv & newhy & & & & \\
\hline 3 & Case 7b Batch 1 & 35 & newhy & newhy & newhy & & & & \\
\hline 3 & Case 7b Batch 1 & 36 & newhy & newhy & & & & & \\
\hline 3 & Case 7b Batch 1 & 37 & newhv & newhy & & & & & \\
\hline 3 & Case 7b Batch 1 & 38 & newhv & newhy & & & & & \\
\hline 3 & Case $7 \mathrm{~b}$ Batch 1 & 39 & newhv & newhv & & & & & \\
\hline 3 & Case $7 \mathrm{~b}$ Batch 1 & 40 & newhy & & & & & & \\
\hline 3 & Case 7b Batch 1 & 41 & newhy & & & & & & \\
\hline 3 & Case 7b Batch 1 & 42 & newhv & & & & & newlv Neph & \\
\hline 3 & Case 7b Batch 1 & 43 & newhv & & & Neph & Neph & newlv Neph & \\
\hline 3 & Case 7b Batch 1 & 44 & & & & Neph & Neph & newlv Neph & Neph \\
\hline 3 & Case $7 \mathrm{~b}$ Batch 1 & 45 & & & Neph & Neph & Neph & newlv Neph & Neph \\
\hline 3 & Case 7b Batch 1 & 46 & Neph & Neph & Neph & Neph & Neph & newlv Neph & Neph \\
\hline 3 & Case 7b Batch 1 & 47 & Neph & Neph & Neph & TL Neph & Neph & newlv Neph & Neph \\
\hline 3 & Case $7 \mathrm{~b}$ Batch 1 & 48 & Neph & Neph & Neph & TL Neph & Neph & newlv Neph & Neph \\
\hline 3 & Case 7b Batch 1 & 49 & Neph & Neph & Neph & TL Neph & TL Neph & TL newlv Neph & Neph \\
\hline 3 & Case $7 \mathrm{~b}$ Batch 1 & 50 & TL Neph & Neph & Neph & TL Neph & TL newlv Neph & TL newlv Neph & Neph \\
\hline 3 & Case $7 \mathrm{~b}$ Batch 1 & 51 & TL Neph & TL Neph & Neph & TL Neph & TL newlv Neph & TL newlv Neph & Neph \\
\hline 3 & Case 7b Batch 1 & 52 & TL Neph & TL Neph & TL Neph & TL Neph & TL newlv Neph & TL newlv Neph & Neph \\
\hline 3 & Case $7 \mathrm{~b}$ Batch 1 & 53 & TL Neph & TL Neph & TL Neph & TL Neph & TL newlv Neph & TL newlv Neph & newlv Neph \\
\hline 3 & Case 7b Batch 1 & 54 & TL Neph & TL Neph & TL Neph & TL Neph & TL newlv Neph & TL newlv Neph & newlv Neph \\
\hline 3 & Case 7b Batch 1 & 55 & TL Neph & TL Neph & TL Neph & TL Neph & TL newlv Neph & TL newlv Neph & TL newlv Neph \\
\hline 3 & Case 7b Batch 1 & 56 & TL Neph & TL Neph & TL Neph & TL Neph & TL newlv Neph & TL newlv Neph & TL newlv Neph \\
\hline 3 & Case 7b Batch 1 & 57 & TL Neph & TL Neph & TL Neph & TL Neph & TL newlv Neph & TL newlv Neph & TL newlv Neph \\
\hline 3 & Case $7 \mathrm{~b}$ Batch 1 & 58 & TL Neph & TL Neph & TL Neph & TL Neph & TL newlv Neph & TL newlv Neph & TL newlv Neph \\
\hline 3 & Case 7b Batch 1 & 59 & TL Neph & TL Neph & TL Neph & TL newlv Neph & TL newlv Neph & TL newlv Neph & TL newlv Neph \\
\hline 3 & Case 7b Batch 1 & 60 & TL Neph & TL Neph & TL Neph & TL newlv Neph & TL newlv Neph & TL newlv Neph & TL newlv Neph \\
\hline 3 & Case $7 \mathrm{~b}$ Batch 2 & 25 & newhy & newhv & newhy & newhy & & & \\
\hline 3 & Case 7b Batch 2 & 26 & newhy & newhy & newhy & newhy & & & \\
\hline 3 & Case $7 \mathrm{~b}$ Batch 2 & 27 & newhv & newhv & newhy & newhy & & & \\
\hline 3 & Case $7 \mathrm{~b}$ Batch 2 & 28 & newhv & newhv & newhv & newhv & & & \\
\hline 3 & Case $7 \mathrm{~b}$ Batch 2 & 29 & newhv & newhv & newhy & newhy & & & \\
\hline 3 & Case $7 \mathrm{~b}$ Batch 2 & 30 & newhv & newhy & newhy & newhy & & & \\
\hline 3 & Case 7b Batch 2 & 31 & newhv & newhv & newhv & newhy & & & \\
\hline 3 & Case 7b Batch 2 & 32 & newhv & newhy & newhy & newhy & & & \\
\hline
\end{tabular}


Exhibit A1. Results for MAR Assessments of the Nominal Stage (part 1)

Constraint not met: neph (nepheline), TL (liquidus temperature), newlv (low viscosity - new model), newhv (high viscosity - new model), and Del GP - durability

\begin{tabular}{|c|c|c|c|c|c|c|c|c|c|}
\hline & & $\mathrm{Na} 2 \mathrm{O}$ & 0.03 & 0.04 & 0.05 & 0.05 & 0.05 & 0.05 & 0.05 \\
\hline Set & Type & $\begin{array}{l}\text { Sludge Loading (\%) } \\
\end{array}$ & 422 & $\begin{array}{ll}\mathrm{d} 1-1 \\
\end{array}$ & 473 & P1-1 & P2-1 & P2-2 & P3-1 \\
\hline 3 & Case 7b Batch 2 & 33 & newhv & newhv & newhy & newhy & & & \\
\hline 3 & Case 7b Batch 2 & 34 & newhv & newhv & newhy & newhy & & & \\
\hline 3 & Case 7b Batch 2 & 35 & newhv & newhv & newhy & & & & \\
\hline 3 & Case 7b Batch 2 & 36 & newhy & newhy & newhy & & & & \\
\hline 3 & $\begin{array}{l}\text { Case 7b Batch } 2 \\
\end{array}$ & 37 & newhv & newhv & newhv & & & & \\
\hline 3 & Case $7 \mathrm{~b}$ Batch 2 & 38 & newhv & newhv & newhv & & & & \\
\hline 3 & Case 7b Batch 2 & 39 & newhv & newhv & newhy & $\mathrm{TL}$ & & & \\
\hline 3 & Case 7b Batch 2 & 40 & newhv & newhv & newhy & $\mathrm{TL}$ & & & \\
\hline 3 & Case 7b Batch 2 & 41 & newhv & newhv & newhv & $\mathrm{TL}$ & $\mathrm{TL}$ & $\mathrm{TL}$ & \\
\hline 3 & Case 7b Batch 2 & 42 & TL newhy & newhv & newhy & $\mathrm{TL}$ & $\mathrm{TL}$ & $\mathrm{TL}$ & \\
\hline 3 & Case 7b Batch 2 & 43 & TL newhy & TL newhy & newhv & $\mathrm{TL}$ & $\mathrm{TL}$ & TL Neph & \\
\hline 3 & Case 7b Batch 2 & 44 & TL newhv & TL newhy & TL newhv & TL Neph & TL Neph & TL Neph & \\
\hline 3 & Case 7b Batch 2 & 45 & TL newhy & TL newhv & TL newhv & TL Neph & TL Neph & TL Neph & Neph \\
\hline 3 & Case 7b Batch 2 & 46 & TL newhy & TL newhy & TL newhv Neph & TL Neph & TL Neph & TL Neph & Neph \\
\hline 3 & Case 7b Batch 2 & 47 & TL newhy & TL newhy Neph & TL newhy Neph & TL Neph & TL Neph & TL Neph & TL Neph \\
\hline 3 & Case 7b Batch 2 & 48 & TL newhy Neph & TL newhy Neph & TL newhy Neph & TL Neph & TL Neph & TL Neph & TL Neph \\
\hline 3 & Case $7 \mathrm{~b}$ Batch 2 & 49 & TL newhv Neph & TL newhv Neph & TL Neph & TL Neph & TL Neph & TL Neph & TL Neph \\
\hline 3 & Case 7b Batch 2 & 50 & TL newhy Neph & TL newhy Neph & TL Neph & TL Neph & TL Neph & TL Neph & TL Neph \\
\hline 3 & Case 7b Batch 2 & 51 & TL newhy Neph & TL newhy Neph & TL Neph & TL Neph & TL Neph & TL Neph & TL Neph \\
\hline 3 & Case 7b Batch 2 & 52 & TL newhv Neph & TL newhv Neph & TL Neph & TL Neph & TL Neph & TL Neph & TL Neph \\
\hline 3 & Case 7b Batch 2 & 53 & TL newhy Neph & TL Neph & TL Neph & TL Neph & TL Neph & TL Neph & TL Neph \\
\hline 3 & Case 7b Batch 2 & 54 & TL newhy Neph & TL Neph & TL Neph & TL Neph & TL Neph & TL Neph & TL Neph \\
\hline 3 & Case 7b Batch 2 & 55 & TL newhy Neph & TL Neph & TL Neph & TL Neph & TL Neph & TL newlv Neph & TL Neph \\
\hline 3 & Case 7b Batch 2 & 56 & TL newhv Neph & TL Neph & TL Neph & TL Neph & TL Neph & TL newlv Neph & TL Neph \\
\hline 3 & Case 7b Batch 2 & 57 & TL Neph & TL Neph & TL Neph & TL Neph & TL Neph & TL newlv Neph & TL Neph \\
\hline 3 & Case 7b Batch 2 & 58 & TL Neph & TL Neph & TL Neph & TL Neph & TL Neph & TL newlv Neph & TL Neph \\
\hline 3 & Case 7b Batch 2 & 59 & TL Neph & TL Neph & TL Neph & TL Neph & TL Neph & TL newlv Neph & TL Neph \\
\hline 3 & Case 7b Batch 2 & 60 & TL Neph & TL Neph & TL Neph & TL Neph & TL Neph & TL newlv Neph & TL Neph \\
\hline 3 & Case $7 \mathrm{~b}$ Blend 1 & 25 & newhy & newhy & newhy & & & & \\
\hline 3 & Case $7 \mathrm{~b}$ Blend 1 & 26 & newhv & newhy & newhy & & & & \\
\hline 3 & Case 7b Blend 1 & 27 & newhy & newhv & newhy & & & & \\
\hline 3 & Case $7 \mathrm{~b}$ Blend 1 & 28 & newhv & newhv & & & & & \\
\hline 3 & Case 7b Blend 1 & 29 & newhv & newhy & & & & & \\
\hline 3 & Case 7b Blend 1 & 30 & newhv & newhv & & & & & \\
\hline 3 & Case $7 \mathrm{~b}$ Blend 1 & 31 & newhy & & & & & & \\
\hline 3 & Case 7b Blend 1 & 32 & newhy & & & & & newlv & \\
\hline 3 & Case $7 \mathrm{~b}$ Blend 1 & 33 & newhv & & & & & newlv & \\
\hline 3 & Case $7 \mathrm{~b}$ Blend 1 & 34 & & & & & & newlv & \\
\hline 3 & Case $7 \mathrm{~b}$ Blend 1 & 35 & & & & & & newlv & \\
\hline 3 & Case $7 \mathrm{~b}$ Blend 1 & 36 & & & & & & newlv & \\
\hline 3 & Case 7b Blend 1 & 37 & & & & & & newlv & \\
\hline 3 & Case $7 \mathrm{~b}$ Blend 1 & 38 & & & & & & newlv & \\
\hline 3 & Case 7b Blend 1 & 39 & & & & & & newlv & \\
\hline 3 & Case $7 \mathrm{~b}$ Blend 1 & 40 & & & & & newlv & newlv & \\
\hline 3 & Case $7 \mathrm{~b}$ Blend 1 & 41 & & & & $\mathrm{TL}$ & newlv & newlv & \\
\hline 3 & Case $7 \mathrm{~b}$ Blend 1 & 42 & & & & $\mathrm{TL}$ & newlv & newlv & newlv \\
\hline 3 & Case $7 \mathrm{~b}$ Blend 1 & 43 & & & & $\mathrm{TL}$ & newlv & newlv & newlv \\
\hline 3 & Case $7 \mathrm{~b}$ Blend 1 & 44 & $\mathrm{TL}$ & & & $\mathrm{TL}$ & TL newlv & TL newlv & newlv \\
\hline 3 & Case 7b Blend 1 & 45 & $\mathrm{TL}$ & $\mathrm{TL}$ & & $\mathrm{TL}$ & $\begin{array}{l}\text { TL newlv } \\
\end{array}$ & $\begin{array}{l}\text { TL newlv } \\
\end{array}$ & newlv \\
\hline 3 & Case $7 \mathrm{~b}$ Blend 1 & 46 & $\mathrm{TL}$ & $\mathrm{TL}$ & $\mathrm{TL}$ & $\mathrm{TL}$ & TL newlv & TL newlv & newlv \\
\hline 3 & Case 7b Blend 1 & 47 & $\mathrm{TL}$ & $\mathrm{TL}$ & $\mathrm{TL}$ & $\mathrm{TL}$ & TL newlv & TL newlv & newlv \\
\hline 3 & Case $7 \mathrm{~b}$ Blend 1 & 48 & $\mathrm{TL}$ & $\mathrm{TL}$ & $\mathrm{TL}$ & TL newlv & TL newlv & TL newlv Neph & newlv \\
\hline
\end{tabular}


Exhibit A1. Results for MAR Assessments of the Nominal Stage (part 1)

Constraint not met: neph (nepheline), TL (liquidus temperature), newlv (low viscosity - new model), newhv (high viscosity - new model), and Del GP - durability

\begin{tabular}{|c|c|c|c|c|c|c|c|c|c|}
\hline & & $\mathrm{Na} 2 \mathrm{O}$ & 0.03 & 0.04 & 0.05 & 0.05 & 0.05 & 0.05 & 0.05 \\
\hline Set & Type & $\begin{array}{l}\text { Sludge Loading }(\%) \\
\end{array}$ & 422 & d1-1 & 473 & P1-1 & P2-1 & P2-2 & P3-1 \\
\hline 3 & Case $7 \mathrm{~b}$ Blend 1 & 49 & $\mathrm{TL}$ & $\mathrm{TL}$ & $\mathrm{TL}$ & TL newlv & TL newlv Neph & TL newlv Neph & TL newlv \\
\hline 3 & Case $7 \mathrm{~b}$ Blend 1 & 50 & $\mathrm{TL}$ & $\mathrm{TL}$ & $\mathrm{TL}$ & TL newlv Neph & TL newlv Neph & TL newlv Neph & TL newlv Neph \\
\hline 3 & Case 7b Blend 1 & 51 & $\mathrm{TL}$ & $\mathrm{TL}$ & TL Neph & TL newlv Neph & TL newly Neph & TL newlv Neph & TL newlv Neph \\
\hline 3 & Case $7 \mathrm{~b}$ Blend 1 & 52 & $\mathrm{TL}$ & TL Neph & TL Neph & TL newlv Neph & TL newlv Neph & TL newlv Neph & TL newlv Neph \\
\hline 3 & Case $7 \mathrm{~b}$ Blend 1 & 53 & TL Neph & TL Neph & TL newlv Neph & TL newlv Neph & TL newlv Neph & TL newlv Neph & TL newlv Neph \\
\hline 3 & Case $7 \mathrm{~b}$ Blend 1 & 54 & TL Neph & TL Neph & TL newlv Neph & TL newlv Neph & TL newlv Neph & TL newlv Neph & TL newlv Neph \\
\hline 3 & Case $7 \mathrm{~b}$ Blend 1 & 55 & TL Neph & TL newlv Neph & TL newlv Neph & TL newlv Neph & TL newlv Neph & TL newlv Neph & TL newlv Neph \\
\hline 3 & Case $7 \mathrm{~b}$ Blend 1 & 56 & TL newlv Neph & TL newlv Neph & TL newlv Neph & TL newlv Neph & TL newlv Neph & TL newlv Neph & TL newlv Neph \\
\hline 3 & Case $7 \mathrm{~b}$ Blend 1 & 57 & TL newlv Neph & TL newlv Neph & TL newlv Neph & TL newlv Neph & TL newlv Neph & TL newlv Neph & TL newlv Neph \\
\hline 3 & Case $7 \mathrm{~b}$ Blend 1 & 58 & TL newlv Neph & TL newlv Neph & TL newlv Neph & TL newlv Neph & TL newlv Neph & TL newlv Neph & TL newlv Neph \\
\hline 3 & Case 7b Blend 1 & 59 & TL newlv Neph & TL newly Neph & TL newlv Neph & TL newly Neph & TL newlv Neph & TL newlv Neph & TL newlv Neph \\
\hline 3 & Case $7 \mathrm{~b}$ Blend 1 & 60 & TL newlv Neph & TL newlv Neph & TL newlv Neph & TL newlv Neph & TL newlv Neph & TL newlv Neph & TL newlv Neph \\
\hline 3 & Case $7 \mathrm{~b}$ Blend 2 & 25 & newhy & newhy & newhy & newhy & & & \\
\hline 3 & Case $7 \mathrm{~b}$ Blend 2 & 26 & newhv & newhv & newhy & newhy & & & \\
\hline 3 & Case $7 \mathrm{~b}$ Blend 2 & 27 & newhv & newhv & newhv & newhy & & & \\
\hline 3 & Case $7 \mathrm{~b}$ Blend 2 & 28 & newhv & newhv & newhy & & & & \\
\hline 3 & Case $7 \mathrm{~b}$ Blend 2 & 29 & newhy & newhy & newhy & & & & \\
\hline 3 & Case $7 \mathrm{~b}$ Blend 2 & 30 & newhv & newhv & newhy & & & & \\
\hline 3 & Case 7b Blend 2 & 31 & newhy & newhy & newhy & & & & \\
\hline 3 & Case $7 \mathrm{~b}$ Blend 2 & 32 & newhy & newhy & newhy & & & & \\
\hline 3 & Case $7 \mathrm{~b}$ Blend 2 & 33 & newhv & newhv & newhy & & & & \\
\hline 3 & Case $7 \mathrm{~b}$ Blend 2 & 34 & newhv & newhv & newhy & & & & \\
\hline 3 & Case $7 \mathrm{~b}$ Blend 2 & 35 & newhv & newhv & newhy & & & & \\
\hline 3 & Case $7 \mathrm{~b}$ Blend 2 & 36 & newhy & newhy & newhy & & & & \\
\hline 3 & Case $7 \mathrm{~b}$ Blend 2 & 37 & newhv & newhv & newhy & & & & \\
\hline 3 & Case $7 \mathrm{~b}$ Blend 2 & 38 & newhv & newhv & newhy & & & & \\
\hline 3 & Case $7 \mathrm{~b}$ Blend 2 & 39 & newhv & newhv & newhy & & & & \\
\hline 3 & Case $7 \mathrm{~b}$ Blend 2 & 40 & newhv & newhy & newhy & $\mathrm{TL}$ & & & \\
\hline 3 & Case $7 \mathrm{~b}$ Blend 2 & 41 & newhv & newhv & & $\mathrm{TL}$ & & & \\
\hline 3 & Case $7 \mathrm{~b}$ Blend 2 & 42 & TL newhv & newhy & & $\mathrm{TL}$ & $\mathrm{TL}$ & TL & \\
\hline 3 & Case $7 \mathrm{~b}$ Blend 2 & 43 & TL newhy & TL newhv & & $\mathrm{TL}$ & $\mathrm{TL}$ & $\mathrm{TL}$ & \\
\hline 3 & Case $7 \mathrm{~b}$ Blend 2 & 44 & TL newhv & TL newhy & & $\mathrm{TL}$ & $\mathrm{TL}$ & TL Neph & \\
\hline 3 & Case $7 \mathrm{~b}$ Blend 2 & 45 & TL newhy & TL & $\mathrm{TL}$ & TL Neph & $\begin{array}{l}\text { TL Neph } \\
\end{array}$ & TL Neph & \\
\hline 3 & Case $7 \mathrm{~b}$ Blend 2 & 46 & TL newhy & $\mathrm{TL}$ & $\mathrm{TL}$ & TL Neph & TL Neph & TL Neph & Neph \\
\hline 3 & Case $7 \mathrm{~b}$ Blend 2 & 47 & TL newhy & $\mathrm{TL}$ & TL Neph & TL Neph & TL Neph & TL newlv Neph & Neph \\
\hline 3 & Case $7 \mathrm{~b}$ Blend 2 & 48 & TL newhv & TL Neph & TL Neph & TL Neph & TL Neph & TL newlv Neph & TL Neph \\
\hline 3 & Case $7 \mathrm{~b}$ Blend 2 & 49 & TL Neph & TL Neph & TL Neph & TL Neph & TL Neph & TL newlv Neph & TL Neph \\
\hline 3 & Case $7 \mathrm{~b}$ Blend 2 & 50 & TL Neph & TL Neph & TL Neph & TL Neph & TL Neph & TL newlv Neph & TL Neph \\
\hline 3 & Case $7 \mathrm{~b}$ Blend 2 & 51 & TL Neph & TL Neph & TL Neph & TL Neph & TL Neph & TL newlv Neph & TL Neph \\
\hline 3 & Case $7 \mathrm{~b}$ Blend 2 & 52 & $\begin{array}{l}\text { TL Neph } \\
\end{array}$ & TL Neph & TL Neph & TL Neph & TL Neph & TL newlv Neph & TL Neph \\
\hline 3 & Case $7 \mathrm{~b}$ Blend 2 & 53 & TL Neph & TL Neph & TL Neph & TL Neph & TL Neph & TL newlv Neph & TL Neph \\
\hline 3 & Case $7 \mathrm{~b}$ Blend 2 & 54 & TL Neph & TL Neph & TL Neph & TL Neph & TL Neph & TL newlv Neph & TL Neph \\
\hline 3 & Case $7 \mathrm{~b}$ Blend 2 & 55 & TL Neph & TL Neph & TL Neph & TL Neph & TL newlv Neph & TL newlv Neph & TL Neph \\
\hline 3 & Case $7 \mathrm{~b}$ Blend 2 & 56 & TL Neph & TL Neph & TL Neph & TL Neph & TL newlv Neph & TL newlv Neph & TL Neph \\
\hline 3 & Case $7 \mathrm{~b}$ Blend 2 & 57 & TL Neph & TL Neph & TL Neph & TL Neph & TL newlv Neph & TL newlv Neph & TL newlv Neph \\
\hline 3 & Case $7 \mathrm{~b}$ Blend 2 & 58 & TL Neph & TL Neph & TL Neph & TL Neph & TL newlv Neph & TL newlv Neph & TL newlv Neph \\
\hline 3 & Case $7 \mathrm{~b}$ Blend 2 & 59 & TL Neph & TL Neph & TL Neph & TL Neph & TL newlv Neph & TL newlv Neph & TL newlv Neph \\
\hline 3 & Case $7 \mathrm{~b}$ Blend 2 & 60 & TL Neph & TL Neph & TL Neph & TL Neph & $\begin{array}{l}\text { TL newlv Neph } \\
\end{array}$ & TL newlv Neph & TL newlv Neph \\
\hline
\end{tabular}


Exhibit A1. Results for MAR Assessments of the Nominal Stage (part 2)

Constraint not met: neph (nepheline), TL (liquidus temperature), newlv (low viscosity - new model), newhv (high viscosity - new model), and Del GP - durability

\begin{tabular}{|c|c|c|c|c|c|c|c|c|c|}
\hline & & $\begin{array}{l}\mathrm{Na} 2 \mathrm{O} \\
\end{array}$ & 0.05 & 0.06 & 0.06 & 0.06 & 0.06 & 0.06 & 0.07 \\
\hline Set & Type & Sludge Loading (\%) & $\mathrm{t} 1-1$ & 202 & 432 & P2-3 & P2-4 & P3-2 & 460 \\
\hline 1 & Case 15B - Batch 1 & 25 & newhv & newhv & newhv & & & & newhv \\
\hline 1 & Case 15B - Batch 1 & 26 & newhy & newhv & newhy & & & & newhv \\
\hline 1 & Case 15B - Batch 1 & 27 & newhy & newhy & newhv & & & & newhv \\
\hline 1 & Case 15B - Batch 1 & 28 & newhy & newhy & newhy & & & & newhv \\
\hline 1 & Case 15B - Batch 1 & 29 & newhv & newhv & newhv & & & & newhy \\
\hline 1 & Case 15B - Batch 1 & 30 & newhv & newhv & newhv & & & & newhv \\
\hline 1 & Case 15B - Batch 1 & 31 & newhv & newhv & newhv & & & & newhv \\
\hline 1 & Case 15B - Batch 1 & 32 & newhv & newhv & newhv & & & & newhv \\
\hline 1 & Case 15B - Batch 1 & 33 & newhv & newhv & newhv & & & & newhv \\
\hline 1 & Case 15B - Batch 1 & 34 & newhv & newhv & newhv & & & & newhv \\
\hline$\frac{1}{1}$ & Case 15B - Batch 1 & 35 & newhy & newhy & newhy & & & & newhv \\
\hline 1 & Case 15B - Batch 1 & 36 & newhv & newhv & newhv & & & & newhv \\
\hline 1 & Case 15B - Batch 1 & 37 & newhv & newhv & newhv & & & & newhv \\
\hline$\frac{1}{1}$ & Case 15B - Batch 1 & 38 & newhy & newhy & newhv & & Neph & & newhv \\
\hline 1 & Case 15B - Batch 1 & 39 & newhy & newhv & newhy & & Neph & & newhv \\
\hline 1 & Case 15B - Batch 1 & 40 & newhv & newhv & newhv & Neph & Neph & Neph & newhv Neph \\
\hline 1 & Case 15B - Batch 1 & 41 & newhv & newhv Neph & newhv Neph & Neph & Neph & Neph & newhv Neph \\
\hline$\frac{1}{1}$ & Case 15B - Batch 1 & $\frac{71}{42}$ & newhv Neph & newhy Neph & newhv Neph & Neph & Neph & Neph & newhv Neph \\
\hline 1 & Case 15B - Batch 1 & 43 & newhv Neph & newhv Neph & newhv Neph & Neph & Neph & Neph & newhv Neph \\
\hline 1 & $\begin{array}{l}\text { Case 15B - Batch } 1 \\
\end{array}$ & 44 & newhv Neph & newhy Neph & newhv Neph & Neph & Neph & Neph & newhv Neph \\
\hline 1 & Case 15B - Batch 1 & 45 & newhv Neph & newhv Neph & newhv Neph & Neph & Neph & Neph & newhv Neph \\
\hline 1 & Case 15B - Batch 1 & 46 & newhv Neph & newhv Neph & newhv Neph & Neph & Neph & Neph & newhv Neph \\
\hline 1 & Case 15B - Batch 1 & 47 & newhv Neph & newhv Neph & newhv Neph & Neph & Neph & Neph & newhv Neph \\
\hline 1 & Case 15B - Batch 1 & 48 & newhv Neph & newhv Neph & newhv Neph & Neph & Neph & Neph & newhv Neph \\
\hline$\frac{1}{1}$ & Case 15B - Batch 1 & 49 & newhv Neph & newhy Neph & newhv Neph & Neph & Neph & Neph & newhv Neph \\
\hline 1 & Case 15B - Batch 1 & 50 & newhv Neph & newhv Neph & newhv Neph & Neph & Neph & Neph & newhv Neph \\
\hline 1 & Case 15B - Batch 1 & 51 & newhv Neph & newhv Neph & newhv Neph & Neph & Neph & Neph & newhv Neph \\
\hline$\frac{1}{1}$ & Case 15B - Batch 1 & $\frac{11}{52}$ & newhv Neph & newhv Neph & newhv Neph & Neph & Neph & Neph & newhv Neph \\
\hline 1 & Case 15B - Batch 1 & 53 & newhv Neph & newhy Neph & newhv Neph & Neph & Neph & Neph & newhv Neph \\
\hline 1 & Case 15B - Batch 1 & 54 & newhv Neph & newhv Neph & newhv Neph & Neph & Neph & Neph & newhv Neph \\
\hline 1 & Case 15B - Batch 1 & 55 & newhv Neph & newhv Neph & newhv Neph & Neph & Neph & Neph & newhv Neph \\
\hline$\frac{1}{1}$ & Case 15B - Batch 1 & 56 & newhv Neph & TL newhv Neph & newhv Neph & Neph & Neph & Neph & newhv Neph \\
\hline 1 & Case 15B - Batch 1 & 57 & TL newhv Neph & TL newhv Neph & newhv Neph & Neph & TL Neph & Neph & newhv Neph \\
\hline 1 & Case 15B - Batch 1 & 58 & TL newhv Neph & TL newhv Neph & newhv Neph & Neph & TL Neph & Neph & newhv Neph \\
\hline 1 & Case 15B - Batch 1 & 59 & TL newhv Neph & TL newhy Neph & newhv Neph & TL Neph & TL Neph & Neph & newhv Neph \\
\hline$\frac{1}{1}$ & Case 15B - Batch 1 & 60 & TL newhv Neph & TL newhv Neph & newhv Neph & TL Neph & TL Neph & Neph & newhv Neph \\
\hline 1 & Case 15B - Batch 2 & 25 & newhv & newhv & newhy & & & & newhv \\
\hline 1 & Case 15B - Batch 2 & 26 & newhv & newhy & newhv & & & & newhv \\
\hline$\frac{1}{1}$ & Case 15B - Batch 2 & 27 & newhy & newhy & newhv & & & & newhy \\
\hline 1 & Case 15B - Batch 2 & 28 & newhy & newhy & newhy & & & & newhy \\
\hline 1 & Case 15B - Batch 2 & 29 & newhv & newhv & newhv & & & & newhv \\
\hline 1 & Case 15B - Batch 2 & 30 & newhv & newhv & newhv & & & & newhv \\
\hline$\frac{1}{1}$ & Case 15B - Batch 2 & 31 & newhy & newhy & newhy & & & & newhy \\
\hline 1 & Case 15B - Batch 2 & 32 & newhv & newhv & newhv & & & & newhv \\
\hline 1 & Case 15B - Batch 2 & 33 & newhv & newhv & newhv & & & & newhv \\
\hline 1 & Case 15B - Batch 2 & 34 & newhv & newhv & newhv & & & & newhv \\
\hline 1 & Case 15B - Batch 2 & 35 & newhy & newhy & newhy & & & & newhv \\
\hline 1 & Case 15B - Batch 2 & 36 & newhv & TL newhv & newhv & & & & newhy \\
\hline 1 & Case 15B - Batch 2 & 37 & newhy & TL newhy & newhv & & & & newhv \\
\hline 1 & Case 15B - Batch 2 & 38 & TL newhy & TL newhy & newhy & & & & newhv \\
\hline 1 & Case 15B - Batch 2 & 39 & TL newhy & TL newhy & newhv & & $\overline{T L}$ & & newhv \\
\hline 1 & Case 15B - Batch 2 & 40 & TL newhy & TL newhy & newhy & $\mathrm{TL}$ & $\mathrm{TL}$ & & newhv \\
\hline
\end{tabular}


Exhibit A1. Results for MAR Assessments of the Nominal Stage (part 2)

Constraint not met: neph (nepheline), TL (liquidus temperature), newlv (low viscosity - new model), newhv (high viscosity - new model), and Del GP - durability

\begin{tabular}{|c|c|c|c|c|c|c|c|c|c|}
\hline & & $\mathrm{Na} 2 \mathrm{O}$ & 0.05 & 0.06 & 0.06 & 0.06 & 0.06 & 0.06 & 0.07 \\
\hline Set & $\begin{array}{c}\text { Type } \\
\end{array}$ & $\begin{array}{l}\text { Sludge Loading (\%) } \\
\end{array}$ & $\mathrm{t} 1-1$ & 202 & 432 & $\begin{array}{l}\mathrm{P} 2-3 \\
\end{array}$ & P2-4 & P3-2 & 460 \\
\hline 1 & Case 15B - Batch 2 & 41 & TL newhy & TL newhy & TL newhy & $\mathrm{TL}$ & $\mathrm{TL}$ & & \\
\hline 1 & Case 15B - Batch 2 & 42 & TL newhy & TL newhy & TL newhy & $\mathrm{TL}$ & $\mathrm{TL}$ & $\mathrm{TL}$ & \\
\hline 1 & Case 15B - Batch 2 & 43 & TL newhy & TL newhy & TL newhy & $\mathrm{TL}$ & TL Neph & $\mathrm{TL}$ & $\mathrm{TL}$ \\
\hline 1 & Case 15B - Batch 2 & 44 & TL newhy & TL newhv & TL newhy & TL Neph & TL Neph & $\mathrm{TL}$ & $\mathrm{TL}$ \\
\hline 1 & Case 15B - Batch 2 & 45 & TL newhy & TL newhy & TL newhy & TL Neph & TL Neph & TL Neph & TL Neph \\
\hline 1 & Case 15B - Batch 2 & 46 & TL newhv Neph & TL newhy Neph & TL newhy Neph & TL Neph & TL Neph & TL Neph & TL Neph \\
\hline 1 & Case 15B - Batch 2 & 47 & TL Neph & TL newhv Neph & TL newhy Neph & TL Neph & TL Neph & TL Neph & TL Neph \\
\hline 1 & Case 15B - Batch 2 & 48 & TL Neph & TL newhy Neph & TL Neph & TL Neph & TL Neph & TL Neph & TL Neph \\
\hline 1 & Case 15B - Batch 2 & 49 & TL Neph & TL newhv Neph & TL Neph & TL Neph & TL Neph & TL Neph & TL Neph \\
\hline 1 & Case 15B - Batch 2 & 50 & TL Neph & TL newhy Neph & TL Neph & TL Neph & TL Neph & TL Neph & TL Neph \\
\hline 1 & $\begin{array}{l}\text { Case 15B - Batch } 2 \\
\end{array}$ & 51 & TL Neph & TL newhy Neph & TL Neph & TL Neph & TL Neph & TL Neph & TL Neph \\
\hline 1 & Case 15B - Batch 2 & 52 & TL Neph & TL newhy Neph & TL Neph & TL Neph & TL Neph & TL Neph & TL Neph \\
\hline 1 & Case 15B - Batch 2 & 53 & TL Neph & TL newhy Neph & TL Neph & TL Neph & TL Neph & TL Neph & TL Neph \\
\hline 1 & Case 15B - Batch 2 & 54 & TL Neph & TL Neph & TL Neph & TL Neph & TL Neph & TL Neph & TL Neph \\
\hline 1 & Case 15B - Batch 2 & 55 & TL Neph & TL Neph & TL Neph & TL Neph & TL newlv Neph & TL Neph & TL Neph \\
\hline 1 & Case 15B - Batch 2 & 56 & TL Neph & TL Neph & TL Neph & TL Neph & TL newlv Neph & TL Neph & TL Neph \\
\hline 1 & Case 15B - Batch 2 & 57 & TL Neph & TL Neph & TL Neph & TL Neph & TL newlv Neph & TL Neph & TL Neph \\
\hline 1 & Case 15B - Batch 2 & 58 & TL Neph & TL Neph & TL Neph & TL Neph & TL newlv Neph & TL Neph & TL Neph \\
\hline 1 & Case 15B - Batch 2 & 59 & TL Neph & TL Neph & TL Neph & TL Neph & TL newlv Neph & TL Neph & TL Neph \\
\hline 1 & $\begin{array}{l}\text { Case 15B - Batch } 2 \\
\end{array}$ & 60 & TL Neph & TL Neph & TL Neph & TL Neph & TL newlv Neph & TL Neph & TL Neph \\
\hline 1 & Case 15B - Blend 1 & 25 & & newhv & & & & & \\
\hline 1 & Case 15B - Blend 1 & 26 & & newhv & & & & & \\
\hline 1 & Case 15B - Blend 1 & 27 & & newhy & & & & & \\
\hline 1 & Case 15B - Blend 1 & 28 & & & & & newlv & & \\
\hline 1 & $\begin{array}{l}\text { Case 15B - Blend } 1 \\
\end{array}$ & 29 & & & & & newlv & & \\
\hline 1 & Case 15B - Blend 1 & 30 & & & & & newlv & & \\
\hline 1 & Case 15B - Blend 1 & 31 & & & & & newlv & & \\
\hline 1 & Case 15B - Blend 1 & 32 & & & & & newlv & & \\
\hline 1 & Case 15B - Blend 1 & 33 & & & & & newlv & & \\
\hline 1 & Case 15B - Blend 1 & 34 & & & & & newlv & & \\
\hline 1 & $\begin{array}{l}\text { Case 15B - Blend } 1 \\
\end{array}$ & 35 & & & & & newlv & & \\
\hline 1 & $\begin{array}{l}\text { Case 15B - Blend } 1 \\
\end{array}$ & 36 & & & & & newlv & & \\
\hline 1 & Case 15B - Blend 1 & 37 & & & & & newlv & & \\
\hline 1 & Case 15B - Blend 1 & 38 & & & & newlv & newlv & & \\
\hline 1 & Case 15B - Blend 1 & 39 & & & & newlv & newlv & & \\
\hline 1 & $\begin{array}{l}\text { Case 15B - Blend } 1 \\
\end{array}$ & 40 & & & & newlv & newlv & & \\
\hline 1 & Case 15B - Blend 1 & 41 & & $\mathrm{TL}$ & & newlv & newlv & & \\
\hline 1 & Case 15B - Blend 1 & 42 & & $\mathrm{TL}$ & & newlv & newlv & newlv & \\
\hline 1 & Case 15B - Blend 1 & 43 & $\mathrm{TL}$ & $\mathrm{TL}$ & & newlv & newlv & newlv & \\
\hline 1 & $\begin{array}{l}\text { Case } 15 \mathrm{~B} \text { - Blend } 1 \\
\end{array}$ & 44 & $\mathrm{TL}$ & $\mathrm{TL}$ & & newlv & TL newlv & newlv & \\
\hline 1 & Case 15B - Blend 1 & 45 & $\mathrm{TL}$ & $\mathrm{TL}$ & & TL newlv & TL newlv & newlv & \\
\hline 1 & Case 15B - Blend 1 & 46 & $\mathrm{TL}$ & $\mathrm{TL}$ & $\mathrm{TL}$ & TL newlv & TL newlv & newlv & \\
\hline 1 & $\begin{array}{l}\text { Case 15B - Blend } 1 \\
\end{array}$ & 47 & $\mathrm{TL}$ & $\mathrm{TL}$ & $\mathrm{TL}$ & TL newlv & TL newlv & TL newlv & $\overline{\mathrm{TL}}$ \\
\hline 1 & Case 15B - Blend 1 & 48 & $\mathrm{TL}$ & $\mathrm{TL}$ & $\mathrm{TL}$ & TL newlv & TL newlv & TL newlv & $\mathrm{TL}$ \\
\hline 1 & Case 15B - Blend 1 & 49 & $\mathrm{TL}$ & $\mathrm{TL}$ & $\mathrm{TL}$ & TL newly & TL newlv Neph & TL newlv & TL newlv \\
\hline 1 & Case 15B - Blend 1 & 50 & TL newlv & $\mathrm{TL}$ & $\mathrm{TL}$ & TL newly Neph & TL newly Neph & TL newlv & TL newlv \\
\hline 1 & Case 15B - Blend 1 & 51 & TL newlv & TL & TL newlv & TL newly Neph & TL newlv Neph & TL newlv Neph & TL newlv Neph \\
\hline 1 & Case 15B - Blend 1 & 52 & TL newlv Neph & TL newlv Neph & TL newlv Neph & TL newlv Neph & TL newlv Neph & TL newlv Neph & TL newlv Neph \\
\hline 1 & Case 15B - Blend 1 & 53 & TL newlv Neph & TL newlv Neph & TL newlv Neph & TL newlv Neph & TL newlv Neph & TL newlv Neph & TL newlv Neph \\
\hline 1 & Case 15B - Blend 1 & 54 & TL newlv Neph & TL newlv Neph & TL newlv Neph & TL newlv Neph & TL newlv Neph & TL newlv Neph & TL newlv Neph \\
\hline 1 & Case 15B - Blend 1 & 55 & TL newlv Neph & TL newlv Neph & TL newlv Neph & TL newlv Neph & TL newlv Neph & TL newlv Neph & TL newlv Neph \\
\hline 1 & Case 15B - Blend 1 & 56 & TL newlv Neph & TL newlv Neph & TL newlv Neph & TL newlv Neph & TL newlv Neph & TL newlv Neph & TL newlv Neph \\
\hline
\end{tabular}


Exhibit A1. Results for MAR Assessments of the Nominal Stage (part 2)

Constraint not met: neph (nepheline), TL (liquidus temperature), newlv (low viscosity - new model), newhv (high viscosity - new model), and Del GP - durability

\begin{tabular}{|c|c|c|c|c|c|c|c|c|c|}
\hline & & $\mathrm{Na2O}$ & 0.05 & 0.06 & 0.06 & 0.06 & 0.06 & 0.06 & 0.07 \\
\hline Set & Type & Sludge Loading (\%) & $\mathrm{t} 1-1$ & 202 & 432 & P2-3 & P2-4 & P3-2 & 460 \\
\hline 1 & Case 15B - Blend 1 & 57 & TL newlv Neph & TL newlv Neph & TL newlv Neph & TL newlv Neph & TL newlv Neph & TL newlv Neph & TL newlv Neph \\
\hline 1 & Case 15B - Blend 1 & 58 & TL newlv Neph & TL newlv Neph & TL newlv Neph & TL newlv Neph & TL newlv Neph & TL newlv Neph & TL newlv Neph \\
\hline 1 & Case 15B - Blend 1 & 59 & TL newlv Neph & TL newlv Neph & TL newlv Neph & TL newlv Neph & TL newlv Neph & TL newlv Neph & TL newlv Neph \\
\hline 1 & Case 15B - Blend 1 & 60 & TL newlv Neph & TL newlv Neph & TL newlv Neph & TL newlv Neph & TL newlv Neph & TL newlv Neph & TL newlv Neph \\
\hline 1 & Case $15 \mathrm{~B}$ - Blend 2 & 25 & newhv & newhv & newhv & & & & newhv \\
\hline 1 & Case 15B - Blend 2 & 26 & newhv & newhv & newhv & & & & newhv \\
\hline 1 & Case 15B - Blend 2 & 27 & newhv & newhv & newhv & & & & newhv \\
\hline$\frac{1}{1}$ & Case 15B - Blend 2 & 28 & newhy & newhy & newhy & & & & newhy \\
\hline 1 & Case 15B - Blend 2 & 29 & newhv & newhv & newhv & & & & newhv \\
\hline 1 & Case 15B - Blend 2 & 30 & newhv & newhv & newhv & & & & newhv \\
\hline 1 & Case 15B - Blend 2 & 31 & newhv & newhv & newhv & & & & newhv \\
\hline 1 & Case 15B - Blend 2 & 32 & newhv & newhv & newhv & & & & newhv \\
\hline 1 & Case 15B - Blend 2 & 33 & newhv & newhv & newhv & & & & \\
\hline 1 & Case 15B - Blend 2 & 34 & newhv & newhv & newhv & & & & \\
\hline 1 & $\begin{array}{l}\text { Case 15B - Blend } 2 \\
\end{array}$ & 35 & newhy & newhv & newhv & & & & \\
\hline 1 & Case $15 \mathrm{~B}-$ Blend 2 & 36 & newhv & newhv & newhv & & & & \\
\hline 1 & Case 15B - Blend 2 & 37 & newhv & TL newhy & newhv & & & & \\
\hline 1 & Case 15B - Blend 2 & 38 & & TL newhy & newhv & & & & \\
\hline 1 & Case 15B - Blend 2 & 39 & $\mathrm{TL}$ & TL newhy & & & & & \\
\hline 1 & Case 15B - Blend 2 & 40 & $\mathrm{TL}$ & TL newhy & & & $\mathrm{TL}$ & & \\
\hline 1 & Case 15B - Blend 2 & 41 & $\mathrm{TL}$ & TL newhv & & $\mathrm{TL}$ & $\mathrm{TL}$ & & \\
\hline 1 & Case 15B - Blend 2 & 42 & $\mathrm{TL}$ & TL newhy & $\mathrm{TL}$ & $\mathrm{TL}$ & $\mathrm{TL}$ & & \\
\hline 1 & Case 15B - Blend 2 & 43 & $\mathrm{TL}$ & TL newhy & $\mathrm{TL}$ & $\mathrm{TL}$ & $\mathrm{TL}$ & $\overline{T L}$ & TL \\
\hline 1 & Case 15B - Blend 2 & 44 & $\mathrm{TL}$ & TL newhy & $\mathrm{TL}$ & $\mathrm{TL}$ & TL Neph & $\mathrm{TL}$ & $\mathrm{TL}$ \\
\hline 1 & Case 15B - Blend 2 & 45 & $\mathrm{TL}$ & $\mathrm{TL}$ & $\mathrm{TL}$ & TL Neph & TL newlv Neph & $\mathrm{TL}$ & $\mathrm{TL}$ \\
\hline$\frac{1}{1}$ & Case 15B - Blend 2 & 46 & $\mathrm{TL}$ & $\mathrm{TL}$ & $\mathrm{TL}$ & TL Neph & TL newlv Neph & TL Neph & TL Neph \\
\hline 1 & Case 15B - Blend 2 & 47 & TL Neph & TL Neph & TL Neph & TL Neph & TL newlv Neph & TL Neph & TL Neph \\
\hline 1 & Case 15B - Blend 2 & 48 & TL Neph & TL Neph & TL Neph & TL Neph & TL newlv Neph & TL Neph & TL Neph \\
\hline 1 & Case 15B - Blend 2 & 49 & TL Neph & TL Neph & TL Neph & TL Neph & TL newlv Neph & TL Neph & TL Neph \\
\hline 1 & Case 15B - Blend 2 & 50 & TL Neph & TL Neph & TL Neph & TL Neph & TL newlv Neph & TL Neph & TL Neph \\
\hline 1 & Case 15B - Blend 2 & 51 & TL Neph & TL Neph & TL Neph & TL Neph & TL newlv Neph & TL Neph & TL Neph \\
\hline 1 & Case 15B - Blend 2 & 52 & TL Neph & TL Neph & TL Neph & TL Neph & TL newlv Neph & TL Neph & TL Neph \\
\hline$\frac{1}{1}$ & Case 15B - Blend 2 & 53 & TL Neph & TL Neph & TL Neph & TL Neph & TL newlv Neph & TL Neph & TL Neph \\
\hline 1 & Case 15B - Blend 2 & 54 & TL Neph & TL Neph & TL Neph & TL Neph & TL newlv Neph & TL Neph & TL Neph \\
\hline 1 & Case 15B - Blend 2 & 55 & TL Neph & TL Neph & TL Neph & TL Neph & TL newlv Neph & TL Neph & TL Neph \\
\hline 1 & Case 15B - Blend 2 & 56 & TL Neph & TL Neph & TL Neph & TL Neph & TL newlv Neph & TL Neph & TL Neph \\
\hline 1 & Case 15B - Blend 2 & 57 & TL Neph & TL Neph & TL Neph & TL newlv Neph & TL newlv Neph & TL Neph & TL Neph \\
\hline 1 & Case $15 \mathrm{~B}$ - Blend 2 & 58 & TL Neph & TL Neph & TL Neph & TL newlv Neph & TL newlv Neph & TL Neph & TL Neph \\
\hline 1 & Case 15B - Blend 2 & 59 & TL Neph & TL Neph & TL Neph & TL newlv Neph & TL newlv Neph & TL Neph & TL Neph \\
\hline$\frac{1}{1}$ & Case 15B - Blend 2 & 60 & TL Neph & TL Neph & TL Neph & TL newlv Neph & TL newlv Neph & TL Neph & TL Neph \\
\hline 1 & Case 16 - Batch 1 & 25 & $\mathrm{Al} 2 \mathrm{O} 3$ & $\mathrm{Al} 2 \mathrm{O} 3$ & $\mathrm{Al} 2 \mathrm{O} 3$ & $\mathrm{Al} 2 \mathrm{O} 3 \mathrm{R} 2 \mathrm{O}$ & Del Gp newlv Al2O3 R2O & $\mathrm{Al} 2 \mathrm{O} 3 \mathrm{R} 2 \mathrm{O}$ & $\mathrm{Al} 2 \mathrm{O} 3 \mathrm{R} 2 \mathrm{O}$ \\
\hline 1 & Case 16-Batch 1 & 26 & $\mathrm{Al} 2 \mathrm{O} 3$ & $\mathrm{Al} 2 \mathrm{O} 3$ & $\mathrm{Al} 2 \mathrm{O} 3$ & Del Gp newlv Al2O3 R2O & Del Gp newlv Al2O3 R2O & Del Gp Al2O3 R2O & $\mathrm{Al} 2 \mathrm{O} 3 \mathrm{R} 2 \mathrm{O}$ \\
\hline 1 & Case 16 - Batch 1 & 27 & $\mathrm{Al} 2 \mathrm{O} 3$ & $\mathrm{Al} 2 \mathrm{O} 3$ & $\mathrm{Al} 2 \mathrm{O} 3 \mathrm{R} 2 \mathrm{O}$ & Del Gp newlv Al2O3 R2O & Del Gp newlv Al2O3 R2O & Del Gp Al2O3 R2O & $\mathrm{Al2O} 3 \mathrm{R} 2 \mathrm{O}$ \\
\hline 1 & Case 16 - Batch 1 & 28 & $\mathrm{~A} 12 \mathrm{O} 3$ & $\mathrm{~A} 12 \mathrm{O} 3$ & $\mathrm{Al} 2 \mathrm{O} 3 \mathrm{R} 2 \mathrm{O}$ & Del Gp newlv Al2O3 R2O & Del Gp newlv Al2O3 R2O & Del Gp Al2O3 R2O & $\mathrm{Al} 2 \mathrm{O} 3 \mathrm{R} 2 \mathrm{O}$ \\
\hline 1 & Case 16- Batch 1 & 29 & $\mathrm{Al} 2 \mathrm{O} 3$ & $\mathrm{Al} 2 \mathrm{O} 3$ & $\mathrm{Al} 2 \mathrm{O} 3 \mathrm{R} 2 \mathrm{O}$ & Del Gp newlv Al2O3 R2O & Del Gp newlv Al2O3 R2O & Del Gp newlv Al2O3 R2O & Del Gp Al2O3 R2O \\
\hline 1 & Case 16 - Batch 1 & 30 & $\mathrm{Al} 2 \mathrm{O} 3$ & $\mathrm{Al} 2 \mathrm{O} 3$ & $\mathrm{Al} 2 \mathrm{O} 3 \mathrm{R} 2 \mathrm{O}$ & Del Gp newlv Al2O3 R2O & Del Gp newlv Al2O3 R2O & Del Gp newlv Al2O3 R2O & Del Gp Al2O3 R2O \\
\hline 1 & Case 16 - Batch 1 & 31 & $\mathrm{Al} 2 \mathrm{O} 3 \mathrm{R} 2 \mathrm{O}$ & $\mathrm{Al} 2 \mathrm{O} 3 \mathrm{R} 2 \mathrm{O}$ & $\mathrm{Al} 2 \mathrm{O} 3 \mathrm{R} 2 \mathrm{O}$ & Del Gp newlv Al2O3 R2O & Del Gp newlv Al2O3 R2O & Del Gp newlv Al2O3 R2O & Del Gp Al2O3 R2O \\
\hline 1 & Case 16- Batch 1 & 32 & $\mathrm{Al} 2 \mathrm{O} 3 \mathrm{R} 2 \mathrm{O}$ & $\mathrm{Al} 2 \mathrm{O} 3 \mathrm{R} 2 \mathrm{O}$ & Del Gp Al2O3 R2O & Del Gp newlv Al2O3 R2O & Del Gp newlv Al2O3 R2O & Del Gp newlv Al2O3 R2O & Del Gp Al2O3 R2O \\
\hline 1 & Case 16-Batch 1 & 33 & $\mathrm{Al} 2 \mathrm{O} 3 \mathrm{R} 2 \mathrm{O}$ & $\mathrm{Al} 2 \mathrm{O} 3 \mathrm{R} 2 \mathrm{O}$ & Del Gp Al2O3 R2O & Del Gp newlv Al2O3 R2O & Del Gp newlv Al2O3 R2O & Del Gp newlv Al2O3 R2O & Del Gp Al2O3 R2O \\
\hline 1 & $\begin{array}{l}\text { Case } 16 \text { - Batch } 1 \\
\end{array}$ & 34 & $\mathrm{Al} 2 \mathrm{O} 3 \mathrm{R} 2 \mathrm{O}$ & Del Gp Al2O3 R2O & Del Gp Al2O3 R2O & Del Gp newlv Al2O3 R2O & Del Gp newlv Al2O3 R2O & Del Gp newlv Al2O3 R2O & Del Gp Al2O3 R2O \\
\hline 1 & Case 16 - Batch 1 & 35 & $\mathrm{Al} 2 \mathrm{O} 3 \mathrm{R} 2 \mathrm{O}$ & Del Gp Al2O3 R2O & Del Gp Al2O3 R2O & Del Gp newlv Al2O3 R2O & Del Gp newlv Al2O3 R2O & Del Gp newlv Al2O3 R2O & Del Gp newlv Al2O3 R2O \\
\hline 1 & Case 16 - Batch 1 & 36 & Del Gp newlv Al2O3 R2O & Del Gp Al2O3 R2O & Del Gp newlv Al2O3 R2O & Del Gp newlv Al2O3 R2O & Del Gp newlv Al2O3 R2O & Del Gp newlv Al2O3 R2O & Del Gp newlv Al2O3 R2O \\
\hline
\end{tabular}


Exhibit A1. Results for MAR Assessments of the Nominal Stage (part 2)

Constraint not met: neph (nepheline), TL (liquidus temperature), newlv (low viscosity - new model), newhv (high viscosity - new model), and Del GP - durability

\begin{tabular}{|c|c|c|c|c|c|c|c|c|c|}
\hline & & $\mathrm{Na2O}$ & 0.05 & 0.06 & 0.06 & 0.06 & 0.06 & 0.06 & $\begin{array}{ll}0.07 \\
\end{array}$ \\
\hline Set & Type & Sludge Loading (\%) & $\frac{0.03}{t 1-1}$ & 202 & 432 & P2-3 & P2-4 & P3-2 & 460 \\
\hline 1 & Case 16 - Batch 1 & 37 & Del Gp newlv Al2O3 R2O & Del Gp Al2O3 R2O & Del Gp newlv Al2O3 R2O & Del Gp newlv Al2O3 R2O & Del Gp newlv Al2O3 R2O & Del Gp newlv Al2O3 R2O & Del Gp newlv Al2O3 R2O \\
\hline 1 & Case 16 - Batch 1 & 38 & Del Gp newlv Al2O3 R2O & Del Gp newlv Al2O3 R2O & Del Gp newlv Al2O3 R2O & Del Gp newlv Al2O3 R2O & Del Gp newlv Al2O3 R2O & Del Gp newlv Al2O3 R2O & Del Gp newlv Al2O3 R2O \\
\hline 1 & Case 16 - Batch 1 & 39 & Del Gp newlv Al2O3 R2O & Del Gp newlv Al2O3 R2O & Del Gp newlv Al2O3 R2O & Del Gp newlv Al2O3 R2O & Del Gp newlv Al2O3 R2O & Del Gp newlv Al203 R2O & Del Gp newlv Al2O3 R2O \\
\hline 1 & Case 16 - Batch 1 & 40 & Del Gp newlv Al2O3 R2O & Del Gp newlv Al2O3 R2O & Del Gp newlv Al2O3 R2O & Del Gp newlv Al2O3 R2O & Del Gp newlv Al2O3 R2O & Del Gp newlv Al2O3 R2O & Del Gp newlv Al2O3 R2O \\
\hline 1 & Case 16 - Batch 1 & 41 & Del Gp newlv Al2O3 R2O & Del Gp newlv Al2O3 R2O & Del Gp newlv Al2O3 R2O & Del Gp newlv Al2O3 R2O & Del Gp newlv Al2O3 R2O & Del Gp newlv Al2O3 R2O & Del Gp newlv Al2O3 R2O \\
\hline 1 & Case 16 - Batch 1 & 42 & Del Gp newlv Al2O3 R2O & Del Gp newlv Al2O3 R2O & Del Gp newlv Al2O3 R2O & Del Gp newlv Al2O3 R2O & Del Gp newlv Al2O3 R2O & Del Gp newlv Al2O3 R2O & Del Gp newlv Al2O3 R2O \\
\hline$\frac{1}{1}$ & Case 16 - Batch 1 & 43 & Del Gp newlv Al2O3 R2O & Del Gp newlv Al2O3 R2O & Del Gp newlv Al2O3 R2O & Del Gp newlv Al2O3 R2O & Del Gp newlv Al2O3 R2O & Del Gp newlv Al2O3 R2O & Del Gp newlv Al2O3 R2O \\
\hline 1 & Case 16 - Batch 1 & 44 & Del Gp newlv Al2O3 R2O & Del Gp newlv Al2O3 R2O & Del Gp newlv Al2O3 R2O & Del Gp newlv Al2O3 R2O & Del Gp newlv Al2O3 R2O & Del Gp newlv Al2O3 R2O & Del Gp newlv Al2O3 R2O \\
\hline 1 & Case 16 - Batch 1 & 45 & Del Gp newlv Al2O3 R2O & Del Gp newlv Al2O3 R2O & Del Gp newlv Al2O3 R2O & Del Gp newlv Al2O3 R2O & Del Gp newlv Al2O3 R2O & Del Gp newlv Al2O3 R2O & Del Gp newlv Al2O3 R2O \\
\hline 1 & Case 16 - Batch 1 & 46 & Del Gp newlv Al2O3 R2O & Del Gp newlv Al2O3 R2O & Del Gp newlv Al2O3 R2O & Del Gp newlv Al2O3 R2O & Del Gp newlv Al2O3 R2O & Del Gp newlv Al2O3 R2O & Del Gp newlv Al2O3 R2O \\
\hline$\frac{1}{1}$ & Case 16 - Batch 1 & 47 & Del Gp newlv Al2O3 R2O & Del Gp newlv Al2O3 R2O & Del Gp newlv Al2O3 R2O & Del Gp newlv Al2O3 R2O & Del Gp newlv Al2O3 R2O & Del Gp newlv Al2O3 R2O & Del Gp newlv Al2O3 R2O \\
\hline 1 & Case 16 - Batch 1 & 48 & Del Gp newlv Al2O3 R2O & Del Gp newlv Al2O3 R2O & Del Gp newlv Al2O3 R2O & Del Gp newlv Al2O3 R2O & Del Gp newlv Al2O3 R2O & Del Gp newlv Al2O3 R2O & Del Gp newlv Al2O3 R2O \\
\hline 1 & Case 16 - Batch 1 & 49 & Del Gp newlv Al2O3 R2O & Del Gp newlv Al2O3 R2O & Del Gp newlv Al2O3 R2O & Del Gp newlv Al2O3 R2O & Del Gp newlv Al2O3 R2O & Del Gp newlv Al2O3 R2O & Del Gp newlv Al2O3 R2O \\
\hline$\frac{1}{1}$ & Case 16 - Batch 1 & 50 & Del Gp newlv Al2O3 R2O & Del Gp newlv Al2O3 R2O & Del Gp newlv Al2O3 R2O & Del Gp newlv Al2O3 R2O & Del Gp newlv Al2O3 R2O & Del Gp newlv Al2O3 R2O & Del Gp newlv Al2O3 R2O \\
\hline 1 & Case 16 - Batch 1 & 51 & Del Gp newlv R2O & Del Gp newlv R2O & Del Gp newlv R2O & Del Gp newlv R2O & Del Gp newlv R2O Neph & Del Gp newlv R2O & Del Gp newlv R2O \\
\hline 1 & Case 16 - Batch 1 & 52 & Del Gp newlv R2O & Del Gp newlv R2O & Del Gp newlv R2O & Del Gp newlv R2O Neph & Del Gp newlv Neph & Del Gp newlv R2O & Del Gp newlv R2O \\
\hline 1 & Case 16 - Batch 1 & 53 & Del Gp newlv R2O & Del Gp TL newlv R2O & Del Gp newlv R2O & Del Gp newlv R2O Neph & Del Gp newlv Neph & Del Gp newlv R2O Neph & Del Gp newlv R2O Neph \\
\hline$\frac{1}{1}$ & Case 16 - Batch 1 & $\frac{3}{54}$ & Del Gp newlv R2O Neph & Del Gp TL newlv R2O Neph & Del Gp newlv R2O Neph & Del Gp newlv R2O Neph & Del Gp newlv Neph & Del Gp newlv R2O Neph & Del Gp newlv R2O Neph \\
\hline 1 & Case 16 - Batch 1 & 55 & Del Gp TL newlv R2O Neph & Del Gp TL newlv R2O Neph & Del Gp newlv R2O Neph & Del Gp newlv R2O Neph & Del Gp newlv Neph & Del Gp newlv R2O Neph & Del Gp newlv R2O Neph \\
\hline 1 & Case 16 - Batch 1 & 56 & Del Gp TL newlv R2O Neph & Del Gp TL newlv R2O Neph & Del Gp newlv R2O Neph & Del Gp newlv R2O Neph & Del Gp newlv Neph & Del Gp newlv R2O Neph & Del Gp newlv R2O Neph \\
\hline$\frac{1}{1}$ & Case 16 - Batch 1 & 57 & Del Gp TL newlv R2O Neph & Del Gp TL newlv R2O Neph & Del Gp newlv R2O Neph & Del Gp newlv Neph & Del Gp newlv Neph & Del Gp newlv R2O Neph & Del Gp newlv R2O Neph \\
\hline 1 & Case 16 - Batch 1 & 58 & Del Gp TL newlv R2O Neph & Del Gp TL newlv R2O Neph & Del Gp TL newlv R2O Neph & Del Gp newlv Neph & Del Gp newlv Neph & Del Gp newlv R2O Neph & Del Gp newlv R2O Neph \\
\hline 1 & Case 16 - Batch 1 & 59 & Del Gp TL newlv Neph & Del Gp TL newlv R2O Neph & Del Gp TL newlv R2O Neph & Del Gp TL newlv Neph & Del Gp TL newlv Neph & Del Gp newlv R2O Neph & Del Gp newlv R2O Neph \\
\hline 1 & Case 16 - Batch 1 & 60 & Del Gp TL newlv Neph & Del Gp TL newlv R2O Neph & Del Gp TL newlv R2O Neph & Del Gp TL newlv Neph & Del Gp TL newlv Neph & Del Gp TL newlv R2O Neph & Del Gp TL newlv R2O Neph \\
\hline$\frac{1}{1}$ & Case 16 - Batch 2 & 25 & newhy & newhv & newhv & & & & newhv \\
\hline 1 & Case 16 - Batch 2 & 26 & newhy & newhv & newhy & & & & newhy \\
\hline 1 & Case 16 - Batch 2 & 27 & newhv & newhv & newhv & & & & newhv \\
\hline$\frac{1}{1}$ & Case 16 - Batch 2 & $\frac{21}{28}$ & newhy & newhy & newhv & & & & newhy \\
\hline 1 & Case 16 - Batch 2 & 29 & newhy & newhy & newhv & & & & newhy \\
\hline 1 & Case 16 - Batch 2 & 30 & newhv & newhv & newhy & & & & newhv \\
\hline 1 & Case 16 - Batch 2 & 31 & newhv & newhv & newhv & & & & newhv \\
\hline$\frac{1}{1}$ & Case 16 - Batch 2 & 32 & newhy & newhy & newhv & & & & newhy \\
\hline 1 & Case 16 - Batch 2 & 33 & newhy & newhy & newhy & & & & newhy \\
\hline 1 & Case 16 - Batch 2 & 34 & newhv & newhv & newhv & & & & newhv \\
\hline 1 & Case 16 - Batch 2 & 35 & newhv & newhv & newhv & & & & newhv \\
\hline$\frac{1}{1}$ & Case 16 - Batch 2 & $\frac{3}{36}$ & newhy & newhy & newhv & & & & newhy \\
\hline 1 & Case 16 - Batch 2 & 37 & newhv & newhv & newhv & & & & newhv \\
\hline 1 & Case 16 - Batch 2 & 38 & newhv & newhv & newhv & & & & newhv \\
\hline$\frac{1}{1}$ & Case 16 - Batch 2 & 39 & newhy & newhy & newhv & & & & newhy \\
\hline 1 & Case 16 - Batch 2 & 40 & newhy & newhy & newhy & & Neph & & newhy \\
\hline 1 & Case 16 - Batch 2 & 41 & newhv & newhy & newhy & Neph & Neph & & newhy \\
\hline 1 & Case 16 - Batch 2 & 42 & newhv & newhv Neph & newhv & Neph & Neph & Neph & newhv Neph \\
\hline$\frac{1}{1}$ & Case 16 - Batch 2 & $\frac{72}{43}$ & newhv Neph & newhv Neph & newhv Neph & Neph & Neph & Neph & newhv Neph \\
\hline 1 & Case 16 - Batch 2 & 44 & newhv Neph & newhv Neph & newhv Neph & Neph & Neph & Neph & newhv Neph \\
\hline 1 & Case 16 - Batch 2 & 45 & newhv Neph & newhv Neph & newhv Neph & Neph & Neph & Neph & newhv Neph \\
\hline 1 & Case 16 - Batch 2 & 46 & newhv Neph & TL newhv Neph & newhv Neph & Neph & Neph & Neph & newhv Neph \\
\hline 1 & Case 16 - Batch 2 & 47 & TL newhv Neph & TL newhv Neph & newhv Neph & Neph & TL Neph & Neph & newhv Neph \\
\hline 1 & Case 16 - Batch 2 & 48 & TL newhv Neph & TL newhv Neph & newhv Neph & Neph & TL Neph & Neph & newhv Neph \\
\hline 1 & Case 16 - Batch 2 & 49 & TL newhv Neph & TL newhv Neph & newhv Neph & TL Neph & TL Neph & Neph & Neph \\
\hline 1 & Case 16 - Batch 2 & 50 & TL newhv Neph & TL newhv Neph & newhv Neph & TL Neph & TL Neph & Neph & Neph \\
\hline 1 & Case 16 - Batch 2 & 51 & TL newhv Neph & TL newhv Neph & TL newhy Neph & TL Neph & TL Neph & Neph & Neph \\
\hline 1 & Case 16 - Batch 2 & 52 & TL newhy Neph & TL newhy Neph & TL newhy Neph & TL Neph & TL Neph & TL Neph & TL Neph \\
\hline
\end{tabular}


Exhibit A1. Results for MAR Assessments of the Nominal Stage (part 2)

Constraint not met: neph (nepheline), TL (liquidus temperature), newlv (low viscosity - new model), newhv (high viscosity - new model), and Del GP - durability

\begin{tabular}{|c|c|c|c|c|c|c|c|c|c|}
\hline & & $\begin{array}{l}\mathrm{Na} 2 \mathrm{O} \\
\end{array}$ & 0.05 & 0.06 & 0.06 & 0.06 & 0.06 & 0.06 & 0.07 \\
\hline Set & Type & Sludge Loading (\%) & t1-1 & 202 & 432 & P2-3 & P2-4 & P3-2 & 460 \\
\hline 1 & Case 16 - Batch 2 & 53 & TL newhv Neph & TL newhv Neph & TL newhv Neph & TL Neph & TL Neph & TL Neph & TL Neph \\
\hline 1 & Case 16 - Batch 2 & 54 & TL newhy Neph & TL newhy Neph & TL newhy Neph & TL Neph & TL Neph & TL Neph & TL Neph \\
\hline 1 & Case 16 - Batch 2 & 55 & TL Neph & TL newhv Neph & TL newhv Neph & TL Neph & TL Neph & TL Neph & TL Neph \\
\hline 1 & Case 16 - Batch 2 & 56 & TL Neph & TL newhv Neph & TL Neph & TL Neph & TL Neph & TL Neph & TL Neph \\
\hline 1 & Case 16 - Batch 2 & 57 & TL Neph & TL newhv Neph & TL Neph & TL Neph & TL Neph & TL Neph & TL Neph \\
\hline 1 & Case 16 - Batch 2 & 58 & TL Neph & TL newhv Neph & TL Neph & TL Neph & TL Neph & TL Neph & TL Neph \\
\hline 1 & Case 16 - Batch 2 & 59 & TL Neph & TL newhv Neph & TL Neph & TL Neph & TL Neph & TL Neph & TL Neph \\
\hline 1 & Case 16 - Batch 2 & 60 & TL Neph & TL newhv Neph & TL Neph & TL Neph & TL Neph & TL Neph & TL Neph \\
\hline 1 & Case 16 - Blend 1 & 25 & & newhv & & & & & \\
\hline 1 & Case 16 - Blend 1 & 26 & & & & & & & \\
\hline$\frac{1}{1}$ & Case 16 - Blend 1 & 27 & & & & & newlv & & \\
\hline 1 & Case 16 - Blend 1 & 28 & & & & & newlv & & \\
\hline 1 & Case 16 - Blend 1 & 29 & & & & & newlv & & \\
\hline$\frac{1}{1}$ & Case 16 - Blend 1 & 30 & & & & & newlv & & \\
\hline 1 & Case 16 - Blend 1 & 31 & & & & & newlv & & \\
\hline 1 & Case 16 - Blend 1 & 32 & & & & & newlv & & \\
\hline 1 & Case 16 - Blend 1 & 33 & & & & & newlv & & \\
\hline$\frac{1}{1}$ & Case 16 - Blend 1 & 34 & & & & & newlv & & \\
\hline 1 & Case 16 - Blend 1 & 35 & & $\mathrm{TL}$ & & & newlv & & \\
\hline 1 & Case 16 - Blend 1 & 36 & & $\mathrm{TL}$ & & newlv & newlv & & \\
\hline 1 & Case 16 - Blend 1 & 37 & $\mathrm{TL}$ & $\mathrm{TL}$ & & newlv & newlv & & \\
\hline 1 & Case 16 - Blend 1 & 38 & $\mathrm{TL}$ & $\mathrm{TL}$ & & newlv & newlv & & \\
\hline 1 & Case 16 - Blend 1 & 39 & $\mathrm{TL}$ & $\mathrm{TL}$ & & TL newlv & TL newlv & & \\
\hline 1 & Case 16 - Blend 1 & 40 & $\mathrm{TL}$ & $\mathrm{TL}$ & $\mathrm{TL}$ & TL newlv & TL newlv & newlv & \\
\hline 1 & Case 16 - Blend 1 & 41 & $\mathrm{TL}$ & $\mathrm{TL}$ & $\mathrm{TL}$ & TL newlv & TL newly & TL newlv & $\mathrm{TL}$ \\
\hline 1 & Case 16 - Blend 1 & 42 & $\mathrm{TL}$ & $\mathrm{TL}$ & $\mathrm{TL}$ & TL newlv & TL newlv & TL newlv & $\mathrm{TL}$ \\
\hline 1 & Case 16 - Blend 1 & 43 & $\mathrm{TL}$ & $\mathrm{TL}$ & $\mathrm{TL}$ & TL newlv & TL newlv & TL newlv & $\mathrm{TL}$ \\
\hline$\frac{1}{1}$ & Case 16 - Blend 1 & $\frac{73}{44}$ & $\mathrm{TL}$ & $\mathrm{TL}$ & $\frac{1 \mathrm{TL}}{\mathrm{TL}}$ & TL newlv & TL newlv & TL newlv & $\mathrm{TL}$ \\
\hline 1 & Case 16 - Blend 1 & 45 & $\mathrm{TL}$ & $\mathrm{TL}$ & $\mathrm{TL}$ & TL newlv & TL newlv & TL newlv & $\mathrm{TL}$ \\
\hline 1 & Case 16 - Blend 1 & 46 & $\mathrm{TL}$ & $\mathrm{TL}$ & $\mathrm{TL}$ & TL newlv & TL newlv & TL newlv & $\mathrm{TL}$ \\
\hline 1 & Case 16 - Blend 1 & 47 & $\mathrm{TL}$ & $\mathrm{TL}$ & $\mathrm{TL}$ & TL newlv & TL newlv & TL newlv & TL newlv \\
\hline$\frac{1}{1}$ & Case 16 - Blend 1 & 48 & TL newlv & $\mathrm{TL}$ & $\mathrm{TL}$ & TL newlv & TL newlv & TL newlv & TL newlv \\
\hline$\frac{1}{1}$ & Case 16 - Blend 1 & 49 & TL newly & $\mathrm{TL}$ & TL newlv & TL newlv & TL newlv & TL newlv & TL newlv \\
\hline 1 & Case 16 - Blend 1 & 50 & TL newlv & TL newlv & TL newlv & TL newlv & TL newlv & TL newlv & TL newlv \\
\hline 1 & Case 16 - Blend 1 & 51 & TL newlv & TL newlv & TL newlv & TL newlv & TL newlv & TL newlv & TL newlv \\
\hline$\frac{1}{1}$ & Case 16 - Blend 1 & 52 & TL newlv & TL newlv & TL newlv & TL newlv & TL newlv & TL newlv & TL newlv \\
\hline 1 & Case 16 - Blend 1 & 53 & TL newlv & TL newlv & TL newlv & TL newlv & TL newly & TL newlv & TL newlv \\
\hline 1 & Case 16 - Blend 1 & 54 & TL newlv & TL newlv & TL newlv & TL newlv & TL newlv Neph & TL newlv & TL newlv \\
\hline 1 & Case 16 - Blend 1 & 55 & TL newlv & TL newlv & TL newlv & TL newly & TL newlv Neph & TL newlv & TL newlv \\
\hline 1 & Case 16 - Blend 1 & 56 & TL newlv & TL newlv & TL newlv & TL newlv Neph & TL newlv Neph & TL newlv Neph & TL newlv \\
\hline 1 & Case 16 - Blend 1 & 57 & TL newlv & TL newlv Neph & TL newlv Neph & TL newlv Neph & TL newlv Neph & TL newlv Neph & TL newlv Neph \\
\hline 1 & Case 16 - Blend 1 & 58 & TL newlv Neph & TL newlv Neph & TL newlv Neph & TL newlv Neph & TL newlv Neph & TL newlv Neph & TL newlv Neph \\
\hline$\frac{1}{1}$ & Case 16 - Blend 1 & 59 & TL newlv Neph & TL newlv Neph & TL newlv Neph & TL newlv Neph & TL newlv Neph & TL newlv Neph & TL newlv Neph \\
\hline 1 & Case 16 - Blend 1 & 60 & TL newlv Neph & TL newlv Neph & TL newlv Neph & TL newlv Neph & TL newlv Neph & TL newlv Neph & TL newlv Neph \\
\hline 1 & Case 16 - Blend 2 & 25 & newhv & newhv & newhv & & & & newhv \\
\hline 1 & Case 16 - Blend 2 & 26 & newhv & newhv & newhy & & & & newhv \\
\hline 1 & Case 16 - Blend 2 & 27 & newhy & newhy & newhv & & & & newhv \\
\hline 1 & Case 16 - Blend 2 & 28 & newhv & newhv & newhv & & & & newhy \\
\hline 1 & Case 16 - Blend 2 & 29 & newhv & newhv & newhv & & & & newhv \\
\hline 1 & Case 16 - Blend 2 & 30 & newhy & newhy & newhy & & & & newhv \\
\hline 1 & Case 16 - Blend 2 & 31 & newhv & newhv & newhv & & & & newhv \\
\hline 1 & Case 16 - Blend 2 & 32 & newhv & newhv & newhy & & & & newhv \\
\hline
\end{tabular}


Exhibit A1. Results for MAR Assessments of the Nominal Stage (part 2)

Constraint not met: neph (nepheline), TL (liquidus temperature), newlv (low viscosity - new model), newhv (high viscosity - new model), and Del GP - durability

\begin{tabular}{|c|c|c|c|c|c|c|c|c|c|}
\hline & & $\mathrm{Na} 2 \mathrm{O}$ & 0.05 & 0.06 & 0.06 & 0.06 & 0.06 & 0.06 & 0.07 \\
\hline Set & Type & Sludge Loading (\%) & $\mathrm{t} 1 \mathrm{-1}$ & 202 & 432 & P2-3 & P2-4 & P3-2 & 460 \\
\hline 1 & Case 16 - Blend 2 & 33 & newhv & newhy & newhy & & & & \\
\hline 1 & Case 16 - Blend 2 & 34 & newhv & newhv & newhv & & & & \\
\hline 1 & Case 16 - Blend 2 & 35 & newhy & newhy & newhy & & & & \\
\hline 1 & Case 16 - Blend 2 & 36 & newhy & newhy & newhy & & & & \\
\hline 1 & $\begin{array}{l}\text { Case } 16 \text { - Blend } 2 \\
\end{array}$ & 37 & newhv & newhv & newhv & & & & \\
\hline 1 & Case 16 - Blend 2 & 38 & newhv & newhv & newhy & & & & \\
\hline 1 & Case 16 - Blend 2 & 39 & & newhv & & & & & \\
\hline 1 & Case 16 - Blend 2 & 40 & & newhv & & & & & \\
\hline 1 & Case 16 - Blend 2 & 41 & & TL newhy & & & & & \\
\hline 1 & Case 16 - Blend 2 & 42 & & TL newhy & & & Neph & & \\
\hline 1 & Case 16 - Blend 2 & 43 & $\mathrm{TL}$ & TL newhy & & & Neph & & \\
\hline 1 & Case 16 - Blend 2 & 44 & $\mathrm{TL}$ & TL newhy & & Neph & TL Neph & Neph & \\
\hline 1 & Case 16 - Blend 2 & 45 & $\mathrm{TL}$ & TL Neph & Neph & TL Neph & TL Neph & Neph & Neph \\
\hline 1 & Case $16-$ Blend 2 & 46 & TL Neph & TL Neph & Neph & TL Neph & TL newly Neph & Neph & Neph \\
\hline 1 & Case 16 - Blend 2 & 47 & TL Neph & TL Neph & TL Neph & TL Neph & TL newlv Neph & TL Neph & Neph \\
\hline 1 & Case 16 - Blend 2 & 48 & TL Neph & TL Neph & TL Neph & TL Neph & TL newlv Neph & TL Neph & TL Neph \\
\hline 1 & Case 16 - Blend 2 & 49 & TL Neph & TL Neph & TL Neph & TL Neph & TL newlv Neph & TL Neph & TL Neph \\
\hline 1 & Case 16 - Blend 2 & 50 & TL Neph & TL Neph & TL Neph & TL Neph & TL newlv Neph & TL Neph & TL Neph \\
\hline 1 & Case 16 - Blend 2 & 51 & TL Neph & TL Neph & TL Neph & TL Neph & TL newlv Neph & TL Neph & TL Neph \\
\hline 1 & Case 16 - Blend 2 & 52 & TL Neph & TL Neph & TL Neph & TL Neph & TL newlv Neph & TL Neph & TL Neph \\
\hline 1 & Case 16 - Blend 2 & 53 & TL Neph & TL Neph & TL Neph & TL Neph & TL newlv Neph & TL Neph & TL Neph \\
\hline 1 & Case 16 - Blend 2 & 54 & TL Neph & TL Neph & TL Neph & TL Neph & TL newlv Neph & TL Neph & TL Neph \\
\hline 1 & Case 16 - Blend 2 & 55 & TL Neph & TL Neph & TL Neph & TL Neph & TL newlv Neph & TL Neph & TL Neph \\
\hline 1 & Case 16 - Blend 2 & 56 & TL Neph & TL Neph & TL Neph & TL Neph & TL newlv Neph & TL Neph & TL Neph \\
\hline 1 & Case 16 - Blend 2 & 57 & TL Neph & TL Neph & TL Neph & TL Neph & TL newly Neph & TL Neph & TL Neph \\
\hline 1 & Case 16 - Blend 2 & 58 & TL Neph & TL Neph & TL Neph & TL newlv Neph & TL newlv Neph & TL Neph & TL Neph \\
\hline 1 & Case 16 - Blend 2 & 59 & TL Neph & TL Neph & TL Neph & TL newlv Neph & TL newlv Neph & TL Neph & TL Neph \\
\hline 1 & Case 16 - Blend 2 & 60 & TL Neph & TL Neph & TL Neph & TL newlv Neph & TL newlv Neph & TL Neph & TL Neph \\
\hline 1 & Case 7 Batch 1 & 25 & newhv & newhv & newhv & & & & newhv \\
\hline 1 & Case 7 Batch 1 & 26 & newhy & newhy & newhv & & & & newhv \\
\hline 1 & Case 7 Batch 1 & 27 & newhv & newhv & newhv & & & & newhv \\
\hline 1 & Case 7 Batch 1 & 28 & newhv & newhv & newhv & & & & newhv \\
\hline 1 & Case 7 Batch 1 & 29 & newhv & newhy & newhv & & & & newhv \\
\hline 1 & Case 7 Batch 1 & 30 & newhy & newhv & newhv & & & & newhv \\
\hline 1 & Case 7 Batch 1 & 31 & newhv & newhv & newhv & & & & \\
\hline 1 & Case 7 Batch 1 & 32 & newhy & newhy & newhv & & & & \\
\hline 1 & Case 7 Batch 1 & 33 & newhv & newhv & newhv & & & & \\
\hline 1 & Case 7 Batch 1 & 34 & newhy & newhv & newhv & & & & \\
\hline 1 & Case 7 Batch 1 & 35 & newhv & newhv & newhv & & & & \\
\hline 1 & Case 7 Batch 1 & 36 & & newhv & & & & & \\
\hline 1 & $\begin{array}{l}\text { Case } 7 \text { Batch } 1 \\
\end{array}$ & 37 & & newhv & & & & & \\
\hline 1 & Case 7 Batch 1 & 38 & & newhv & & & & & \\
\hline 1 & Case 7 Batch 1 & 39 & & newhy & & & & & \\
\hline 1 & Case 7 Batch 1 & 40 & & newhv & & & & & \\
\hline 1 & Case 7 Batch 1 & 41 & & newhv & & & Neph & & \\
\hline 1 & Case 7 Batch 1 & 42 & & & & Neph & Neph & & \\
\hline 1 & Case 7 Batch 1 & 43 & & & & Neph & newlv Neph & Neph & Neph \\
\hline 1 & Case 7 Batch 1 & 44 & & TL Neph & Neph & Neph & newlv Neph & Neph & Neph \\
\hline 1 & Case 7 Batch 1 & 45 & TL Neph & TL Neph & Neph & Neph & newlv Neph & Neph & Neph \\
\hline 1 & Case 7 Batch 1 & 46 & TL Neph & TL Neph & Neph & Neph & TL newlv Neph & Neph & Neph \\
\hline 1 & Case 7 Batch 1 & 47 & TL Neph & TL Neph & Neph & TL Neph & TL newlv Neph & Neph & Neph \\
\hline 1 & $\begin{array}{l}\text { Case } 7 \text { Batch } 1 \\
\end{array}$ & 48 & TL Neph & TL Neph & Neph & TL Neph & TL newlv Neph & Neph & Neph \\
\hline
\end{tabular}


Exhibit A1. Results for MAR Assessments of the Nominal Stage (part 2)

Constraint not met: neph (nepheline), TL (liquidus temperature), newlv (low viscosity - new model), newhv (high viscosity - new model), and Del GP - durability

\begin{tabular}{|c|c|c|c|c|c|c|c|c|c|}
\hline & & " $\mathrm{Na} 2 \mathrm{O}$ & 0.05 & 0.06 & 0.06 & 0.06 & 0.06 & 0.06 & 0.07 \\
\hline Set & Type & Sludge Loading (\%) & $\mathrm{t} 1-1$ & 202 & 432 & P2-3 & P2-4 & P3-2 & 460 \\
\hline 1 & Case 7 Batch 1 & 49 & TL Neph & TL Neph & TL Neph & TL Neph & TL newlv Neph & Neph & Neph \\
\hline 1 & Case 7 Batch 1 & 50 & TL Neph & TL Neph & TL Neph & TL Neph & TL newlv Neph & TL Neph & TL Neph \\
\hline$\frac{1}{1}$ & Case 7 Batch 1 & 51 & TL Neph & TL Neph & TL Neph & TL Neph & TL newlv Neph & TL Neph & TL Neph \\
\hline 1 & Case 7 Batch 1 & 52 & TL Neph & TL Neph & TL Neph & TL Neph & TL newlv Neph & TL Neph & TL Neph \\
\hline 1 & Case 7 Batch 1 & 53 & TL Neph & TL Neph & TL Neph & TL Neph & TL newlv Neph & TL Neph & TL Neph \\
\hline 1 & Case 7 Batch 1 & 54 & TL Neph & TL Neph & TL Neph & TL newlv Neph & TL newlv Neph & TL Neph & TL Neph \\
\hline 1 & Case 7 Batch 1 & 55 & TL Neph & TL Neph & TL Neph & TL newlv Neph & TL newlv Neph & TL Neph & TL Neph \\
\hline$\frac{1}{1}$ & Case 7 Batch 1 & 56 & TL Neph & TL Neph & TL Neph & TL newlv Neph & TL newlv Neph & TL Neph & TL Neph \\
\hline 1 & Case 7 Batch 1 & 57 & TL Neph & TL Neph & TL Neph & TL newlv Neph & TL newlv Neph & TL Neph & TL Neph \\
\hline 1 & Case 7 Batch 1 & 58 & TL Neph & TL Neph & TL Neph & TL newlv Neph & TL newly Neph & TL newlv Neph & TL Neph \\
\hline$\frac{1}{1}$ & Case 7 Batch 1 & 59 & TL Neph & TL Neph & TL Neph & TL newlv Neph & TL newlv Neph & TL newlv Neph & TL Neph \\
\hline 1 & Case 7 Batch 1 & 60 & TL Neph & TL Neph & TL Neph & TL newlv Neph & TL newlv Neph & TL newlv Neph & TL Neph \\
\hline 1 & Case 7 Batch 2 & 25 & newhy & newhy & newhy & & & & newhy \\
\hline$\frac{1}{1}$ & Case 7 Batch 2 & 26 & newhy & newhy & newhy & & & & newhv \\
\hline 1 & Case 7 Batch 2 & 27 & newhv & newhv & newhv & & & & newhv \\
\hline 1 & Case 7 Batch 2 & 28 & newhv & newhv & newhv & & & & newhv \\
\hline 1 & Case 7 Batch 2 & 29 & newhy & newhy & newhy & & & & newhy \\
\hline$\frac{1}{1}$ & Case 7 Batch 2 & 30 & newhy & newhy & newhv & & & & newhy \\
\hline 1 & Case 7 Batch 2 & 31 & newhy & newhy & newhv & & & & newhv \\
\hline 1 & Case 7 Batch 2 & 32 & newhy & newhv & newhy & & & & newhy \\
\hline 1 & Case 7 Batch 2 & 33 & newhv & newhv & newhv & & & & newhv \\
\hline 1 & Case 7 Batch 2 & 34 & newhy & newhy & newhv & & & & newhy \\
\hline 1 & Case 7 Batch 2 & 35 & newhv & newhv & newhv & & & & newhv \\
\hline 1 & Case 7 Batch 2 & 36 & newhv & newhy & newhy & & & & newhv \\
\hline 1 & Case 7 Batch 2 & 37 & newhv & TL newhy & newhv & & & & newhy \\
\hline 1 & Case 7 Batch 2 & 38 & TL newhy & TL newhy & newhy & & & & newhv \\
\hline 1 & Case 7 Batch 2 & 39 & TL newhy & TL newhy & newhv & & $\mathrm{TL}$ & & newhv \\
\hline$\frac{1}{1}$ & Case 7 Batch 2 & 40 & TL newhy & TL newhy & newhv & $\mathrm{TL}$ & $\frac{1 \mathrm{LL}}{\mathrm{TL}}$ & & newhy \\
\hline 1 & Case 7 Batch 2 & 41 & TL newhy & TL newhy & newhv & $\mathrm{TL}$ & $\mathrm{TL}$ & & newhy \\
\hline 1 & Case 7 Batch 2 & 42 & TL newhy & TL newhy & TL newhv & $\mathrm{TL}$ & $\mathrm{TL}$ & $\overline{T L}$ & newhy \\
\hline 1 & Case 7 Batch 2 & 43 & TL newhy & TL newhy & TL newhy & $\mathrm{TL}$ & TL Neph & $\mathrm{TL}$ & TL newhy \\
\hline$\frac{1}{1}$ & Case 7 Batch 2 & 44 & TL newhy & TL newhv & TL newhy & TL Neph & TL Neph & TL Neph & TL newhv \\
\hline$\frac{1}{1}$ & Case 7 Batch 2 & 45 & TL newhy & TL newhy Neph & TL newhy & TL Neph & TL Neph & TL Neph & TL newhy Neph \\
\hline 1 & Case 7 Batch 2 & 46 & TL newhv Neph & TL newhv Neph & TL newhy Neph & TL Neph & TL Neph & TL Neph & TL newhv Neph \\
\hline 1 & Case 7 Batch 2 & 47 & TL newhy Neph & TL newhv Neph & TL newhy Neph & TL Neph & TL Neph & TL Neph & TL Neph \\
\hline$\frac{1}{1}$ & Case 7 Batch 2 & 48 & TL newhv Neph & TL newhv Neph & TL newhv Neph & TL Neph & TL Neph & TL Neph & TL Neph \\
\hline 1 & Case 7 Batch 2 & 49 & TL newhv Neph & TL newhv Neph & TL newhy Neph & TL Neph & TL Neph & TL Neph & TL Neph \\
\hline 1 & Case 7 Batch 2 & 50 & TL newhv Neph & TL newhv Neph & TL newhy Neph & TL Neph & TL Neph & TL Neph & TL Neph \\
\hline 1 & Case 7 Batch 2 & 51 & TL newhv Neph & TL newhv Neph & TL newhy Neph & TL Neph & TL Neph & TL Neph & TL Neph \\
\hline 1 & Case 7 Batch 2 & 52 & TL newhv Neph & TL newhv Neph & TL newhy Neph & TL Neph & TL Neph & TL Neph & TL Neph \\
\hline 1 & Case 7 Batch 2 & 53 & TL Neph & TL newhv Neph & TL newhy Neph & TL Neph & TL Neph & TL Neph & TL Neph \\
\hline 1 & Case 7 Batch 2 & 54 & TL Neph & TL newhv Neph & TL Neph & TL Neph & TL Neph & TL Neph & TL Neph \\
\hline$\frac{1}{1}$ & Case 7 Batch 2 & 55 & TL Neph & TL newhv Neph & TL Neph & TL Neph & TL Neph & TL Neph & TL Neph \\
\hline 1 & Case 7 Batch 2 & 56 & TL Neph & TL newhv Neph & TL Neph & TL Neph & TL Neph & TL Neph & TL Neph \\
\hline 1 & Case 7 Batch 2 & 57 & TL Neph & TL newhv Neph & TL Neph & TL Neph & TL Neph & TL Neph & TL Neph \\
\hline 1 & Case 7 Batch 2 & 58 & TL Neph & TL newhy Neph & TL Neph & TL Neph & TL Neph & TL Neph & TL Neph \\
\hline 1 & Case 7 Batch 2 & 59 & TL Neph & TL newhv Neph & TL Neph & TL Neph & TL Neph & TL Neph & TL Neph \\
\hline 1 & Case 7 Batch 2 & 60 & TL Neph & TL Neph & TL Neph & TL Neph & TL Neph & TL Neph & TL Neph \\
\hline 1 & Case 7 Blend 1 & 25 & & newhv & & & & & \\
\hline 1 & Case 7 Blend 1 & 26 & & newhy & & & & & \\
\hline 1 & Case 7 Blend 1 & 27 & & & & & & & \\
\hline 1 & Case 7 Blend 1 & 28 & & & & & newlv & & \\
\hline
\end{tabular}




\section{Exhibit A1. Results for MAR Assessments of the Nominal Stage (part 2)}

Constraint not met: neph (nepheline), TL (liquidus temperature), newlv (low viscosity - new model), newhv (high viscosity - new model), and Del GP - durability

\begin{tabular}{|c|c|c|c|c|c|c|c|c|c|}
\hline & & $\mathrm{Na} 2 \mathrm{O}$ & 0.05 & 0.06 & 0.06 & 0.06 & 0.06 & 0.06 & 0.07 \\
\hline Set & Type & Sludge Loading (\%) & $\mathrm{t} 1-1$ & 202 & 432 & $\mathrm{P} 2-3$ & P2-4 & P3-2 & 460 \\
\hline 1 & Case 7 Blend 1 & 29 & & & & & newlv & & \\
\hline 1 & Case 7 Blend 1 & 30 & & & & & newlv & & \\
\hline 1 & Case 7 Blend 1 & 31 & & & & & newlv & & \\
\hline 1 & Case 7 Blend 1 & 32 & & & & & newlv & & \\
\hline 1 & Case 7 Blend 1 & 33 & & & & & newlv & & \\
\hline 1 & Case 7 Blend 1 & 34 & & & & & newlv & & \\
\hline 1 & Case 7 Blend 1 & 35 & & & & & newlv & & \\
\hline 1 & Case 7 Blend 1 & 36 & & & & & newlv & & \\
\hline 1 & Case 7 Blend 1 & 37 & & & & & newlv & & \\
\hline 1 & Case 7 Blend 1 & 38 & & & & newlv & newlv & & \\
\hline 1 & Case 7 Blend 1 & 39 & & & & newlv & newlv & & \\
\hline 1 & Case 7 Blend 1 & 40 & & & & newlv & newlv & & \\
\hline 1 & Case 7 Blend 1 & 41 & & $\mathrm{TL}$ & & newlv & newlv & newlv & \\
\hline 1 & Case 7 Blend 1 & 42 & $\mathrm{TL}$ & $\mathrm{TL}$ & & newlv & newlv & newlv & \\
\hline 1 & Case 7 Blend 1 & 43 & $\mathrm{TL}$ & $\mathrm{TL}$ & & newlv & newlv & newlv & \\
\hline 1 & Case 7 Blend 1 & 44 & $\mathrm{TL}$ & $\mathrm{TL}$ & & newlv & TL newly & newlv & \\
\hline 1 & Case 7 Blend 1 & 45 & $\mathrm{TL}$ & $\mathrm{TL}$ & & TL newlv & TL newlv & newlv & \\
\hline 1 & Case 7 Blend 1 & 46 & $\mathrm{TL}$ & $\mathrm{TL}$ & $\mathrm{TL}$ & TL newlv & TL newlv & newlv & \\
\hline 1 & Case 7 Blend 1 & 47 & $\mathrm{TL}$ & $\mathrm{TL}$ & $\mathrm{TL}$ & TL newlv & TL newlv & TL newlv & $\mathrm{TL}$ \\
\hline 1 & Case 7 Blend 1 & 48 & $\mathrm{TL}$ & $\mathrm{TL}$ & $\mathrm{TL}$ & TL newlv & TL newlv & TL newlv & TL newlv \\
\hline 1 & Case 7 Blend 1 & 49 & $\mathrm{TL}$ & $\mathrm{TL}$ & $\mathrm{TL}$ & TL newlv & TL newlv Neph & TL newlv & TL newlv \\
\hline 1 & Case 7 Blend 1 & 50 & TL newlv & $\mathrm{TL}$ & TL newlv & TL newlv Neph & TL newlv Neph & TL newlv & TL newlv \\
\hline 1 & Case 7 Blend 1 & 51 & TL newlv & TL & TL newlv & TL newlv Neph & TL newlv Neph & TL newlv Neph & TL newlv Neph \\
\hline 1 & $\begin{array}{l}\text { Case } 7 \text { Blend } 1 \\
\end{array}$ & 52 & TL newlv Neph & TL newlv Neph & TL newlv Neph & TL newlv Neph & TL newlv Neph & TL newlv Neph & TL newlv Neph \\
\hline 1 & Case 7 Blend 1 & 53 & TL newlv Neph & TL newlv Neph & TL newlv Neph & TL newlv Neph & TL newly Neph & TL newlv Neph & TL newlv Neph \\
\hline 1 & Case 7 Blend 1 & 54 & TL newlv Neph & TL newlv Neph & TL newlv Neph & TL newlv Neph & TL newlv Neph & TL newlv Neph & TL newlv Neph \\
\hline 1 & Case 7 Blend 1 & 55 & TL newlv Neph & TL newlv Neph & TL newlv Neph & TL newlv Neph & TL newlv Neph & TL newlv Neph & TL newlv Neph \\
\hline 1 & Case 7 Blend 1 & 56 & TL newlv Neph & TL newlv Neph & TL newlv Neph & TL newlv Neph & TL newlv Neph & TL newlv Neph & TL newlv Neph \\
\hline 1 & Case 7 Blend 1 & 57 & TL newlv Neph & TL newlv Neph & TL newlv Neph & TL newlv Neph & TL newlv Neph & TL newlv Neph & TL newlv Neph \\
\hline 1 & Case 7 Blend 1 & 58 & TL newlv Neph & TL newlv Neph & TL newlv Neph & TL newlv Neph & TL newlv Neph & TL newlv Neph & TL newlv Neph \\
\hline 1 & Case 7 Blend 1 & 59 & TL newlv Neph & TL newlv Neph & TL newlv Neph & TL newlv Neph & TL newlv Neph & TL newlv Neph & TL newlv Neph \\
\hline 1 & Case 7 Blend 1 & 60 & TL newlv Neph & TL newlv Neph & TL newlv Neph & TL newlv Neph & TL newlv Neph & TL newlv Neph & TL newlv Neph \\
\hline 1 & Case 7 Blend 2 & 25 & newhv & newhy & newhv & & & & newhv \\
\hline 1 & Case 7 Blend 2 & 26 & newhy & newhy & newhy & & & & newhy \\
\hline 1 & Case 7 Blend 2 & 27 & newhv & newhv & newhv & & & & newhv \\
\hline 1 & Case 7 Blend 2 & 28 & newhy & newhy & newhy & & & & newhy \\
\hline 1 & Case 7 Blend 2 & 29 & newhv & newhv & newhv & & & & newhv \\
\hline 1 & Case 7 Blend 2 & 30 & newhv & newhv & newhv & & & & newhv \\
\hline 1 & Case 7 Blend 2 & 31 & newhv & newhv & newhv & & & & newhv \\
\hline 1 & Case 7 Blend 2 & 32 & newhv & newhv & newhv & & & & newhy \\
\hline 1 & $\begin{array}{l}\text { Case } 7 \text { Blend } 2 \\
\end{array}$ & 33 & newhv & newhv & newhv & & & & newhv \\
\hline 1 & Case 7 Blend 2 & 34 & newhv & newhv & newhv & & & & newhv \\
\hline 1 & Case 7 Blend 2 & 35 & newhy & newhy & newhy & & & & \\
\hline 1 & Case 7 Blend 2 & 36 & newhv & newhv & newhy & & & & \\
\hline 1 & Case 7 Blend 2 & 37 & newhv & newhv & newhv & & & & \\
\hline 1 & Case 7 Blend 2 & 38 & newhy & TL newhy & newhv & & & & \\
\hline 1 & Case 7 Blend 2 & 39 & TL newhy & TL newhy & newhy & & & & \\
\hline 1 & Case 7 Blend 2 & 40 & $\mathrm{TL}$ & TL newhy & newhv & & $\mathrm{TL}$ & & \\
\hline 1 & Case 7 Blend 2 & 41 & $\mathrm{TL}$ & TL newhy & & TL & $\mathrm{TL}$ & & \\
\hline 1 & Case 7 Blend 2 & 42 & $\mathrm{TL}$ & TL newhy & & $\mathrm{TL}$ & $\mathrm{TL}$ & & \\
\hline 1 & Case 7 Blend 2 & 43 & $\mathrm{TL}$ & TL newhy & $\mathrm{TL}$ & $\mathrm{TL}$ & TL & $\mathrm{TL}$ & \\
\hline 1 & $\begin{array}{l}\text { Case } 7 \text { Blend } 2 \\
\end{array}$ & 44 & $\mathrm{TL}$ & TL newhy & $\mathrm{TL}$ & $\mathrm{TL}$ & TL Neph & $\mathrm{TL}$ & $\mathrm{TL}$ \\
\hline
\end{tabular}


Exhibit A1. Results for MAR Assessments of the Nominal Stage (part 2)

Constraint not met: neph (nepheline), TL (liquidus temperature), newlv (low viscosity - new model), newhv (high viscosity - new model), and Del GP - durability

\begin{tabular}{|c|c|c|c|c|c|c|c|c|c|}
\hline & & $\begin{array}{l}\mathrm{Na} 2 \mathrm{O} \\
\end{array}$ & 0.05 & 0.06 & 0.06 & 0.06 & 0.06 & 0.06 & $\begin{array}{ll}0.07 \\
\end{array}$ \\
\hline Set & Type & Sludge Loading (\%) & $\frac{t 1-1}{t 1}$ & 202 & 432 & P2-3 & P2-4 & P3-2 & 460 \\
\hline 1 & Case 7 Blend 2 & 45 & $\mathrm{TL}$ & TL newhy & $\mathrm{TL}$ & TL Neph & TL Neph & TL Neph & $\mathrm{TL}$ \\
\hline 1 & Case 7 Blend 2 & 46 & $\mathrm{TL}$ & TL newhy Neph & $\mathrm{TL}$ & TL Neph & TL Neph & TL Neph & TL Neph \\
\hline$\frac{1}{1}$ & Case 7 Blend 2 & $\frac{40}{47}$ & TL Neph & TL Neph & TL Neph & TL Neph & TL Neph & TL Neph & TL Neph \\
\hline 1 & Case 7 Blend 2 & 48 & TL Neph & TL Neph & TL Neph & TL Neph & TL newlv Neph & TL Neph & TL Neph \\
\hline 1 & Case 7 Blend 2 & 49 & TL Neph & TL Neph & TL Neph & TL Neph & TL newlv Neph & TL Neph & TL Neph \\
\hline 1 & Case 7 Blend 2 & 50 & TL Neph & TL Neph & TL Neph & TL Neph & TL newlv Neph & TL Neph & TL Neph \\
\hline$\frac{1}{1}$ & Case 7 Blend 2 & 51 & TL Neph & TL Neph & TL Neph & TL Neph & TL newlv Neph & TL Neph & TL Neph \\
\hline$\frac{1}{1}$ & Case 7 Blend 2 & 52 & TL Neph & TL Neph & TL Neph & TL Neph & TL newlv Neph & TL Neph & TL Neph \\
\hline 1 & Case 7 Blend 2 & 53 & TL Neph & TL Neph & TL Neph & TL Neph & TL newlv Neph & TL Neph & TL Neph \\
\hline 1 & Case 7 Blend 2 & 54 & TL Neph & TL Neph & TL Neph & TL Neph & TL newly Neph & TL Neph & TL Neph \\
\hline$\frac{1}{1}$ & Case 7 Blend 2 & 55 & TL Neph & TL Neph & TL Neph & TL Neph & TL newlv Neph & TL Neph & TL Neph \\
\hline 1 & Case 7 Blend 2 & 56 & TL Neph & TL Neph & TL Neph & TL Neph & TL newlv Neph & TL Neph & TL Neph \\
\hline 1 & Case 7 Blend 2 & 57 & TL Neph & TL Neph & TL Neph & TL Neph & TL newlv Neph & TL Neph & TL Neph \\
\hline$\frac{1}{1}$ & Case 7 Blend 2 & 58 & TL Neph & TL Neph & TL Neph & TL Neph & TL newlv Neph & TL Neph & TL Neph \\
\hline 1 & Case 7 Blend 2 & 59 & TL Neph & TL Neph & TL Neph & TL newlv Neph & TL newlv Neph & TL Neph & TL Neph \\
\hline 1 & Case 7 Blend 2 & 60 & TL Neph & TL Neph & TL Neph & TL newlv Neph & TL newlv Neph & TL Neph & TL Neph \\
\hline 1 & Case 8 - Blend 1 & 25 & newhy & newhy & newhv & & & & \\
\hline$\frac{1}{1}$ & Case 8 - Blend 1 & 26 & & newhv & & & & & \\
\hline 1 & Case 8 - Blend 1 & 27 & & newhv & & & & & \\
\hline 1 & Case 8 - Blend 1 & 28 & & newhy & & & & & \\
\hline 1 & Case 8 - Blend 1 & 29 & & newhv & & & & & \\
\hline 1 & Case 8 - Blend 1 & 30 & & newhv & & & & & \\
\hline 1 & Case 8 - Blend 1 & 31 & & & & & & & \\
\hline 1 & Case 8 - Blend 1 & 32 & & & & & newlv & & \\
\hline 1 & Case 8 - Blend 1 & 33 & & & & & newlv & & \\
\hline 1 & Case 8 - Blend 1 & 34 & & & & & newlv & & \\
\hline 1 & Case 8 - Blend 1 & 35 & & & & & newlv & & \\
\hline$\frac{1}{1}$ & Case 8 - Blend 1 & $\frac{5}{36}$ & & & & & newlv & & \\
\hline 1 & Case 8 - Blend 1 & 37 & & & & & newlv & & \\
\hline 1 & Case 8 - Blend 1 & 38 & & & & & newlv & & \\
\hline 1 & Case 8 - Blend 1 & 39 & & $\mathrm{TL}$ & & & newlv & & \\
\hline$\frac{1}{1}$ & Case 8 - Blend 1 & 40 & & $\mathrm{TL}$ & & & newlv & & \\
\hline$\frac{1}{1}$ & Case 8 - Blend 1 & 41 & $\overline{T L}$ & $\mathrm{TL}$ & & & newlv & & \\
\hline 1 & Case 8 - Blend 1 & 42 & TL & $\mathrm{TL}$ & & newlv & TL newlv & & \\
\hline 1 & Case 8 - Blend 1 & 43 & TL & $\mathrm{TL}$ & & TL newlv & TL newlv & & \\
\hline$\frac{1}{1}$ & Case 8 - Blend 1 & 44 & $\frac{T L}{T L}$ & $\frac{T L}{T L}$ & $\mathrm{TL}$ & TL newlv & TL newlv & & \\
\hline 1 & Case 8 - Blend 1 & 45 & $\mathrm{TL}$ & $\mathrm{TL}$ & $\mathrm{TL}$ & TL newlv & TL newlv & & \\
\hline 1 & Case 8 - Blend 1 & 46 & $\mathrm{TL}$ & $\mathrm{TL}$ & $\mathrm{TL}$ & TL newlv & TL newlv Neph & TL newlv & $\mathrm{TL}$ \\
\hline 1 & Case 8 - Blend 1 & 47 & $\mathrm{TL}$ & $\mathrm{TL}$ & $\mathrm{TL}$ & TL newlv Neph & TL newlv Neph & TL newlv & $\mathrm{TL}$ \\
\hline 1 & Case 8 - Blend 1 & 48 & $\mathrm{TL}$ & $\mathrm{TL}$ & $\mathrm{TL}$ & TL newlv Neph & TL newlv Neph & TL newlv Neph & TL Neph \\
\hline 1 & Case 8 - Blend 1 & 49 & TL Neph & TL Neph & TL Neph & TL newlv Neph & TL newlv Neph & TL newlv Neph & TL Neph \\
\hline$\frac{1}{1}$ & Case 8 - Blend 1 & 50 & TL Neph & TL Neph & TL Neph & TL newlv Neph & TL newlv Neph & TL newlv Neph & TL Neph \\
\hline$\frac{1}{1}$ & Case 8 - Blend 1 & 51 & TL Neph & TL Neph & TL Neph & TL newlv Neph & TL newlv Neph & TL newlv Neph & TL Neph \\
\hline 1 & Case 8 - Blend 1 & 52 & TL Neph & TL Neph & TL Neph & TL newlv Neph & TL newlv Neph & TL newlv Neph & TL Neph \\
\hline 1 & Case 8 - Blend 1 & 53 & TL Neph & TL Neph & TL Neph & TL newlv Neph & TL newlv Neph & TL newlv Neph & TL newlv Neph \\
\hline 1 & Case 8 - Blend 1 & 54 & TL newlv Neph & TL Neph & TL Neph & TL newly Neph & TL newlv Neph & TL newlv Neph & TL newlv Neph \\
\hline 1 & Case 8 - Blend 1 & 55 & TL newlv Neph & TL Neph & TL newlv Neph & TL newlv Neph & TL newlv Neph & TL newlv Neph & TL newlv Neph \\
\hline 1 & Case 8 - Blend 1 & 56 & TL newlv Neph & TL newlv Neph & TL newlv Neph & TL newlv Neph & TL newlv Neph & TL newlv Neph & TL newlv Neph \\
\hline 1 & Case 8 - Blend 1 & 57 & TL newlv Neph & TL newlv Neph & TL newlv Neph & TL newlv Neph & TL newlv Neph & TL newlv Neph & TL newlv Neph \\
\hline 1 & Case 8 - Blend 1 & 58 & TL newlv Neph & TL newlv Neph & TL newlv Neph & TL newlv Neph & TL newlv Neph & TL newlv Neph & TL newlv Neph \\
\hline 1 & Case 8 - Blend 1 & 59 & TL newlv Neph & TL newlv Neph & TL newlv Neph & TL newlv Neph & TL newlv Neph & TL newlv Neph & TL newlv Neph \\
\hline 1 & Case 8 - Blend 1 & 60 & TL newlv Neph & TL newlv Neph & TL newlv Neph & TL newlv Neph & TL newlv Neph & TL newlv Neph & TL newlv Neph \\
\hline
\end{tabular}


Exhibit A1. Results for MAR Assessments of the Nominal Stage (part 2)

Constraint not met: neph (nepheline), TL (liquidus temperature), newlv (low viscosity - new model), newhv (high viscosity - new model), and Del GP - durability

\begin{tabular}{|c|c|c|c|c|c|c|c|c|c|}
\hline & & $\mathrm{Na} 2 \mathrm{O}$ & 0.05 & 0.06 & 0.06 & 0.06 & 0.06 & 0.06 & 0.07 \\
\hline Set & $\begin{array}{c}\text { Type } \\
\end{array}$ & Sludge Loading (\%) & $\mathrm{t} 1 \mathrm{-1}$ & 202 & 432 & $\mathrm{P} 2-3$ & P2-4 & P3-2 & 460 \\
\hline 1 & Case 8 and $8 \mathrm{a}$ - Batch 1 & 25 & newhv & newhy & newhv & & & & newhv \\
\hline 1 & Case 8 and $8 \mathrm{a}$ - Batch 1 & 26 & newhv & newhv & newhv & & & & newhv \\
\hline 1 & Case 8 and $8 \mathrm{a}-$ - Batch 1 & 27 & newhy & newhv & newhv & & & & newhv \\
\hline 1 & Case 8 and $8 \mathrm{a}$ - Batch 1 & 28 & newhy & newhy & newhy & & & & \\
\hline 1 & Case 8 and $8 \mathrm{a}$ - Batch 1 & 29 & newhv & newhv & newhv & & & & \\
\hline 1 & Case 8 and $8 \mathrm{a}$ - Batch 1 & 30 & newhv & newhv & newhv & & & & \\
\hline 1 & Case 8 and $8 \mathrm{a}-$ - Batch 1 & 31 & newhv & newhv & newhv & & & & \\
\hline 1 & Case 8 and $8 \mathrm{a}$ - Batch 1 & 32 & newhv & newhv & newhy & & & & \\
\hline 1 & Case 8 and $8 \mathrm{a}$ - Batch 1 & 33 & & newhy & & & & & \\
\hline 1 & Case 8 and $8 \mathrm{a}-$ - Batch 1 & 34 & & newhy & & & & & \\
\hline 1 & Case 8 and $8 a$ - Batch 1 & 35 & & newhy & & & & & \\
\hline 1 & Case 8 and $8 \mathrm{a}$ - Batch 1 & 36 & & newhv & & & & & \\
\hline 1 & Case 8 and $8 \mathrm{a}$ - Batch 1 & 37 & & newhv & & & & & \\
\hline 1 & Case 8 and $8 \mathrm{a}$ - Batch 1 & 38 & & newhy & & & & & \\
\hline 1 & Case 8 and $8 \mathrm{a}$ - Batch 1 & 39 & & $\mathrm{TL}$ & & & & & \\
\hline 1 & Case 8 and $8 \mathrm{a}$ - Batch 1 & 40 & $\mathrm{TL}$ & $\mathrm{TL}$ & & & newlv & & \\
\hline 1 & Case 8 and $8 \mathrm{a}$ - Batch 1 & 41 & $\mathrm{TL}$ & $\mathrm{TL}$ & & & TL newlv & & \\
\hline 1 & Case 8 and $8 a$ - Batch 1 & 42 & $\mathrm{TL}$ & $\mathrm{TL}$ & & & TL newlv & & \\
\hline 1 & Case 8 and $8 \mathrm{a}$ - Batch 1 & 43 & $\mathrm{TL}$ & $\mathrm{TL}$ & & $\mathrm{TL}$ & TL newlv Neph & & \\
\hline 1 & Case 8 and $8 \mathrm{a}$ - Batch 1 & 44 & $\mathrm{TL}$ & $\mathrm{TL}$ & $\mathrm{TL}$ & TL Neph & TL newlv Neph & & \\
\hline 1 & Case 8 and $8 \mathrm{a}-$ - Batch 1 & 45 & $\mathrm{TL}$ & $\mathrm{TL}$ & $\mathrm{TL}$ & TL Neph & TL newlv Neph & TL Neph & TL Neph \\
\hline 1 & Case 8 and $8 \mathrm{a}-$ Batch 1 & 46 & TL Neph & TL Neph & TL Neph & TL Neph & TL newlv Neph & TL Neph & TL Neph \\
\hline 1 & Case 8 and $8 \mathrm{a}$ - Batch 1 & 47 & TL Neph & TL Neph & TL Neph & TL Neph & TL newlv Neph & TL Neph & TL Neph \\
\hline 1 & Case 8 and $8 \mathrm{a}$ - Batch 1 & 48 & TL Neph & TL Neph & TL Neph & TL Neph & TL newlv Neph & TL Neph & TL Neph \\
\hline 1 & Case 8 and $8 \mathrm{a}$ - Batch 1 & 49 & TL Neph & TL Neph & TL Neph & TL Neph & TL newly Neph & TL Neph & TL Neph \\
\hline 1 & Case 8 and $8 \mathrm{a}$ - Batch 1 & 50 & TL Neph & TL Neph & TL Neph & TL Neph & TL newlv Neph & TL Neph & TL Neph \\
\hline 1 & Case 8 and $8 \mathrm{a}-$ - Batch 1 & 51 & TL Neph & TL Neph & TL Neph & TL newlv Neph & TL newlv Neph & TL Neph & TL Neph \\
\hline 1 & Case 8 and $8 \mathrm{a}$ - Batch 1 & 52 & TL Neph & TL Neph & TL Neph & TL newlv Neph & TL newlv Neph & TL Neph & TL Neph \\
\hline 1 & Case 8 and $8 \mathrm{a}-$ - Batch 1 & 53 & TL Neph & TL Neph & TL Neph & TL newlv Neph & TL newlv Neph & TL Neph & TL Neph \\
\hline 1 & Case 8 and $8 \mathrm{a}$ - Batch 1 & 54 & TL Neph & TL Neph & TL Neph & TL newlv Neph & TL newly Neph & TL Neph & TL Neph \\
\hline 1 & Case 8 and $8 \mathrm{a}$ - Batch 1 & 55 & TL Neph & TL Neph & TL Neph & TL newlv Neph & TL newlv Neph & TL newlv Neph & TL Neph \\
\hline 1 & Case 8 and $8 \mathrm{a}-$ - Batch 1 & 56 & TL Neph & TL Neph & TL Neph & TL newlv Neph & TL newlv Neph & TL newlv Neph & TL Neph \\
\hline 1 & Case 8 and $8 \mathrm{a}$ - Batch 1 & 57 & TL Neph & TL Neph & TL Neph & TL newlv Neph & TL newlv Neph & TL newlv Neph & TL Neph \\
\hline 1 & Case 8 and $8 \mathrm{a}$ - Batch 1 & 58 & TL Neph & TL Neph & TL Neph & TL newlv Neph & TL newlv Neph & TL newlv Neph & TL Neph \\
\hline 1 & Case 8 and $8 \mathrm{a}$ - Batch 1 & 59 & TL Neph & TL Neph & TL Neph & TL newlv Neph & TL newlv Neph & TL newlv Neph & TL Neph \\
\hline 1 & Case 8 and $8 \mathrm{a}$ - Batch 1 & 60 & TL Neph & TL Neph & TL Neph & TL newlv Neph & TL newlv Neph & TL newlv Neph & TL Neph \\
\hline 1 & Case $8 \mathrm{~A}$ - Blend 1 & 25 & newhy & newhy & newhy & & & & \\
\hline 1 & $\begin{array}{l}\text { Case } 8 \mathrm{~A} \text { - Blend } 1 \\
\end{array}$ & 26 & newhv & newhy & newhv & & & & \\
\hline 1 & Case $8 \mathrm{~A}$ - Blend 1 & 27 & newhy & newhy & newhy & & & & \\
\hline 1 & Case 8A - Blend 1 & 28 & & newhv & newhv & & & & \\
\hline 1 & Case $8 \mathrm{~A}$ - Blend 1 & 29 & & newhv & & & & & \\
\hline 1 & Case $8 \mathrm{~A}$ - Blend 1 & 30 & & newhv & & & & & \\
\hline 1 & Case $8 \mathrm{~A}$ - Blend 1 & 31 & & newhy & & & & & \\
\hline 1 & Case $8 \mathrm{~A}$ - Blend 1 & 32 & & newhv & & & & & \\
\hline 1 & Case $8 \mathrm{~A}$ - Blend 1 & 33 & & newhy & & & & & \\
\hline 1 & Case $8 \mathrm{~A}$ - Blend 1 & 34 & & & & & & & \\
\hline 1 & Case $8 \mathrm{~A}$ - Blend 1 & 35 & & & & & newlv & & \\
\hline 1 & Case $8 \mathrm{~A}$ - Blend 1 & 36 & & & & & newlv & & \\
\hline 1 & Case $8 \mathrm{~A}$ - Blend 1 & 37 & & & & & newlv & & \\
\hline 1 & Case $8 \mathrm{~A}$ - Blend 1 & 38 & & & & & newlv & & \\
\hline 1 & Case 8A - Blend 1 & 39 & & $\mathrm{TL}$ & & & newlv & & \\
\hline 1 & $\begin{array}{l}\text { Case } 8 \mathrm{~A} \text { - Blend } 1 \\
\end{array}$ & 40 & & $\mathrm{TL}$ & & & newlv & & \\
\hline
\end{tabular}


Exhibit A1. Results for MAR Assessments of the Nominal Stage (part 2)

Constraint not met: neph (nepheline), TL (liquidus temperature), newlv (low viscosity - new model), newhv (high viscosity - new model), and Del GP - durability

\begin{tabular}{|c|c|c|c|c|c|c|c|c|c|}
\hline & & $\begin{array}{l}\mathrm{Na} 2 \mathrm{O} \\
\end{array}$ & 0.05 & 0.06 & 0.06 & 0.06 & 0.06 & 0.06 & $\begin{array}{ll}0.07 \\
\end{array}$ \\
\hline Set & Type & Sludge Loading (\%) & $\frac{t 1-1}{t 1}$ & 202 & $\frac{0.06}{432}$ & P2-3 & P2-4 & $\frac{0.0}{\mathrm{P} 3-2}$ & 460 \\
\hline 1 & Case 8A - Blend 1 & 41 & $\mathrm{TL}$ & $\mathrm{TL}$ & & & newlv & & \\
\hline 1 & Case $8 \mathrm{~A}$ - Blend 1 & 42 & $\mathrm{TL}$ & $\mathrm{TL}$ & & & TL newlv & & \\
\hline 1 & Case 8A - Blend 1 & 43 & $\mathrm{TL}$ & $\mathrm{TL}$ & & $\mathrm{TL}$ & TL newlv & & \\
\hline 1 & Case $8 \mathrm{~A}$ - Blend 1 & 44 & $\mathrm{TL}$ & $\mathrm{TL}$ & $\mathrm{TL}$ & $\mathrm{TL}$ & TL newlv & & \\
\hline 1 & Case 8A - Blend 1 & 45 & $\mathrm{TL}$ & $\mathrm{TL}$ & $\mathrm{TL}$ & $\mathrm{TL}$ & TL newlv Neph & $\mathrm{TL}$ & \\
\hline 1 & Case 8A - Blend 1 & 46 & $\mathrm{TL}$ & $\overline{T L}$ & $\mathrm{TL}$ & TL newlv Neph & TL newlv Neph & TL Neph & $\mathrm{TL}$ \\
\hline 1 & Case 8A - Blend 1 & 47 & $\mathrm{TL}$ & TL Neph & $\mathrm{TL}$ & TL newlv Neph & TL newlv Neph & TL Neph & TL Neph \\
\hline$\frac{1}{1}$ & Case $8 \mathrm{~A}$ - Blend 1 & 48 & TL Neph & TL Neph & TL Neph & TL newlv Neph & TL newlv Neph & TL Neph & TL Neph \\
\hline 1 & Case $8 \mathrm{~A}$ - Blend 1 & 49 & TL Neph & TL Neph & TL Neph & TL newlv Neph & TL newlv Neph & TL newlv Neph & TL Neph \\
\hline 1 & Case 8A - Blend 1 & 50 & TL Neph & TL Neph & TL Neph & TL newlv Neph & TL newlv Neph & TL newlv Neph & TL Neph \\
\hline$\frac{1}{1}$ & Case $8 \mathrm{~A}$ - Blend 1 & 51 & TL Neph & TL Neph & TL Neph & TL newlv Neph & TL newlv Neph & TL newlv Neph & TL Neph \\
\hline 1 & Case 8A - Blend 1 & 52 & TL Neph & TL Neph & TL Neph & TL newlv Neph & TL newlv Neph & TL newlv Neph & TL Neph \\
\hline 1 & Case 8A - Blend 1 & 53 & TL Neph & TL Neph & TL Neph & TL newlv Neph & TL newlv Neph & TL newlv Neph & TL Neph \\
\hline$\frac{1}{1}$ & Case $8 \mathrm{~A}$ - Blend 1 & 54 & TL Neph & TL Neph & TL Neph & TL newlv Neph & TL newlv Neph & TL newlv Neph & TL Neph \\
\hline 1 & Case 8A - Blend 1 & 55 & TL Neph & TL Neph & TL Neph & TL newlv Neph & TL newlv Neph & TL newlv Neph & TL Neph \\
\hline 1 & Case $8 \mathrm{~A}$ - Blend 1 & 56 & TL Neph & TL Neph & TL Neph & TL newlv Neph & TL newlv Neph & TL newlv Neph & TL newlv Neph \\
\hline 1 & Case $8 \mathrm{~A}$ - Blend 1 & 57 & TL Neph & TL Neph & TL Neph & TL newlv Neph & TL newlv Neph & TL newlv Neph & TL newlv Neph \\
\hline$\frac{1}{1}$ & Case 8A - Blend 1 & 58 & TL newlv Neph & TL Neph & TL newlv Neph & TL newlv Neph & TL newlv Neph & TL newlv Neph & TL newlv Neph \\
\hline 1 & Case $8 \mathrm{~A}$ - Blend 1 & 59 & TL newlv Neph & TL Neph & TL newlv Neph & TL newlv Neph & TL newlv Neph & TL newlv Neph & TL newlv Neph \\
\hline 1 & Case 8A - Blend 1 & 60 & TL newlv Neph & TL newlv Neph & TL newlv Neph & TL newlv Neph & TL newlv Neph & TL newlv Neph & TL newlv Neph \\
\hline 2 & Case 15 Batch 1 & 25 & newhv & newhv & newhv & newhv & & newhv & newhv \\
\hline 2 & Case 15 Batch 1 & 26 & newhv & newhv & newhv & newhv & & newhv & newhv \\
\hline 2 & Case 15 Batch 1 & 27 & newhv & newhv & newhv & newhv & & newhv & newhy \\
\hline 2 & Case 15 Batch 1 & 28 & newhv & newhv & newhy & newhy & & newhv & newhv \\
\hline 2 & Case 15 Batch 1 & 29 & newhy & newhy & newhv & newhy & & newhy & newhy \\
\hline 2 & Case 15 Batch 1 & 30 & newhv & newhv & newhy & newhy & & newhy & newhy \\
\hline 2 & Case 15 Batch 1 & 31 & newhv & newhv & newhv & newhv & & newhv & newhv \\
\hline$\frac{2}{2}$ & Case 15 Batch 1 & $\frac{11}{32}$ & newhy & newhy & newhv & newhv & & newhy & newhy \\
\hline 2 & Case 15 Batch 1 & 33 & newhv & newhv & newhv & newhv & & newhy & newhv \\
\hline 2 & Case 15 Batch 1 & 34 & newhy & newhv & newhv & newhv & & newhv & newhv \\
\hline 2 & Case 15 Batch 1 & 35 & newhv & newhv & newhv & newhv & & newhv & newhv \\
\hline$\frac{2}{2}$ & Case 15 Batch 1 & 36 & newhy & newhy & newhy & newhv & & newhy & newhy \\
\hline 2 & Case 15 Batch 1 & 37 & newhv & newhv & newhv & newhv & & newhv & newhv \\
\hline 2 & Case 15 Batch 1 & 38 & newhv & newhv & newhv & newhv & & newhv & newhv \\
\hline 2 & Case 15 Batch 1 & 39 & newhv & newhv & newhy & newhy & Neph & newhv & newhv \\
\hline$\frac{2}{2}$ & Case 15 Batch 1 & 40 & newhy & newhy & newhy & newhv & Neph & newhv & newhv \\
\hline 2 & Case 15 Batch 1 & 41 & newhv & newhv & newhv & newhv Neph & Neph & newhv Neph & newhv Neph \\
\hline 2 & Case 15 Batch 1 & 42 & newhv & newhv Neph & newhv Neph & newhv Neph & Neph & newhv Neph & newhv Neph \\
\hline 2 & Case 15 Batch 1 & 43 & newhv Neph & newhv Neph & newhv Neph & newhv Neph & Neph & newhv Neph & newhv Neph \\
\hline 2 & Case 15 Batch 1 & 44 & newhv Neph & TL newhv Neph & newhv Neph & newhv Neph & Neph & newhv Neph & newhv Neph \\
\hline 2 & Case 15 Batch 1 & 45 & newhv Neph & TL newhv Neph & newhv Neph & newhv Neph & TL Neph & newhv Neph & newhv Neph \\
\hline 2 & Case 15 Batch 1 & 46 & TL newhv Neph & TL newhv Neph & newhv Neph & newhv Neph & TL Neph & newhv Neph & newhv Neph \\
\hline$\frac{2}{2}$ & Case 15 Batch 1 & $\frac{40}{47}$ & TL newhv Neph & TL newhv Neph & newhv Neph & newhy Neph & TL Neph & newhv Neph & newhv Neph \\
\hline 2 & Case 15 Batch 1 & 48 & TL newhv Neph & TL newhv Neph & newhv Neph & TL newhv Neph & TL Neph & newhv Neph & newhv Neph \\
\hline 2 & Case 15 Batch 1 & 49 & TL newhv Neph & TL newhv Neph & newhv Neph & TL newhv Neph & TL Neph & newhv Neph & newhv Neph \\
\hline$\frac{2}{2}$ & Case 15 Batch 1 & 50 & TL newhv Neph & TL newhv Neph & TL newhv Neph & TL newhv Neph & TL Neph & TL newhy Neph & newhv Neph \\
\hline 2 & Case 15 Batch 1 & 51 & TL newhv Neph & TL newhv Neph & TL newhy Neph & TL newhv Neph & TL Neph & TL newhv Neph & TL newhv Neph \\
\hline 2 & Case 15 Batch 1 & 52 & TL newhv Neph & TL newhv Neph & TL newhy Neph & TL newhv Neph & TL Neph & TL newhv Neph & TL newhv Neph \\
\hline 2 & Case 15 Batch 1 & 53 & TL newhv Neph & TL newhv Neph & TL newhy Neph & TL newhy Neph & TL Neph & TL newhy Neph & TL newhv Neph \\
\hline 2 & Case 15 Batch 1 & 54 & TL newhv Neph & TL newhv Neph & TL newhy Neph & TL newhv Neph & TL newhv Neph & TL newhv Neph & TL newhv Neph \\
\hline 2 & Case 15 Batch 1 & 55 & TL newhv Neph & TL newhv Neph & TL newhy Neph & TL newhv Neph & TL newhv Neph & TL newhv Neph & TL newhv Neph \\
\hline 2 & Case 15 Batch 1 & 56 & TL newhy Neph & TL newhy Neph & TL newhy Neph & TL newhv Neph & TL newhy Neph & TL newhy Neph & TL newhy Neph \\
\hline
\end{tabular}


Exhibit A1. Results for MAR Assessments of the Nominal Stage (part 2)

Constraint not met: neph (nepheline), TL (liquidus temperature), newlv (low viscosity - new model), newhv (high viscosity - new model), and Del GP - durability

\begin{tabular}{|c|c|c|c|c|c|c|c|c|c|}
\hline & & Na2O & 0.05 & 0.06 & 0.06 & 0.06 & 0.06 & 0.06 & 0.07 \\
\hline Set & Type & Sludge Loading (\%) & $\mathrm{t} 1-1$ & 202 & 432 & P2-3 & P2-4 & P3-2 & 460 \\
\hline 2 & Case 15 Batch 1 & 57 & TL newhy Neph & TL newhy Neph & TL newhy Neph & TL newhv Neph & TL newhy Neph & TL newhy Neph & TL newhy Neph \\
\hline 2 & Case 15 Batch 1 & 58 & TL newhv Neph & TL newhv Neph & TL newhv Neph & TL newhy Neph & TL newhv Neph & TL newhv Neph & TL newhy Neph \\
\hline 2 & Case 15 Batch 1 & 59 & TL newhy Neph & TL newhy Neph & TL newhy Neph & TL newhv Neph & TL newhy Neph & TL newhy Neph & TL newhv Neph \\
\hline 2 & Case 15 Batch 1 & 60 & TL newhv Neph & TL newhv Neph & TL newhy Neph & TL newhy Neph & TL newhy Neph & TL newhy Neph & TL newhy Neph \\
\hline 2 & Case 15 Batch 2 & 25 & newhv & newhv & newhv & & & & newhv \\
\hline 2 & Case 15 Batch 2 & 26 & newhv & newhv & newhy & & & & \\
\hline 2 & Case 15 Batch 2 & 27 & newhv & newhv & newhv & & & & \\
\hline 2 & Case 15 Batch 2 & 28 & newhv & newhv & newhy & & & & \\
\hline 2 & Case 15 Batch 2 & 29 & newhv & newhy & newhv & & & & \\
\hline 2 & Case 15 Batch 2 & 30 & newhy & newhv & newhv & & & & \\
\hline 2 & Case 15 Batch 2 & 31 & & newhy & & & & & \\
\hline 2 & Case 15 Batch 2 & 32 & & newhv & & & & & \\
\hline 2 & Case 15 Batch 2 & 33 & & newhv & & & & & \\
\hline 2 & Case 15 Batch 2 & 34 & & newhy & & & & & \\
\hline 2 & Case 15 Batch 2 & 35 & & newhv & & & & & \\
\hline 2 & Case 15 Batch 2 & 36 & & newhv & & & & & \\
\hline 2 & Case 15 Batch 2 & 37 & & & & & newlv & & \\
\hline 2 & Case 15 Batch 2 & 38 & & & & & newlv & & \\
\hline 2 & Case 15 Batch 2 & 39 & & & & & newlv & & \\
\hline 2 & Case 15 Batch 2 & 40 & & $\mathrm{TL}$ & & & newlv & & \\
\hline 2 & Case 15 Batch 2 & 41 & & $\mathrm{TL}$ & & & newlv & & \\
\hline 2 & Case 15 Batch 2 & 42 & $\mathrm{TL}$ & $\mathrm{TL}$ & & & newlv & & \\
\hline 2 & $\begin{array}{l}\text { Case } 15 \text { Batch } 2 \\
\end{array}$ & 43 & $\mathrm{TL}$ & $\mathrm{TL}$ & & & TL newlv Neph & & \\
\hline 2 & Case 15 Batch 2 & 44 & $\mathrm{TL}$ & $\mathrm{TL}$ & & TL Neph & TL newlv Neph & & \\
\hline 2 & Case 15 Batch 2 & 45 & $\mathrm{TL}$ & TL Neph & & TL Neph & TL newly Neph & Neph & Neph \\
\hline 2 & Case 15 Batch 2 & 46 & TL Neph & TL Neph & TL Neph & TL Neph & TL newlv Neph & TL Neph & Neph \\
\hline 2 & Case 15 Batch 2 & 47 & TL Neph & TL Neph & TL Neph & TL Neph & TL newlv Neph & TL Neph & TL Neph \\
\hline 2 & Case 15 Batch 2 & 48 & TL Neph & TL Neph & TL Neph & TL Neph & TL newlv Neph & TL Neph & TL Neph \\
\hline 2 & Case 15 Batch 2 & 49 & TL Neph & TL Neph & TL Neph & TL newlv Neph & TL newlv Neph & TL Neph & TL Neph \\
\hline 2 & Case 15 Batch 2 & 50 & TL Neph & TL Neph & TL Neph & TL newlv Neph & TL newly Neph & TL Neph & TL Neph \\
\hline 2 & Case 15 Batch 2 & 51 & TL Neph & TL Neph & TL Neph & TL newlv Neph & TL newlv Neph & TL Neph & TL Neph \\
\hline 2 & Case 15 Batch 2 & 52 & TL Neph & TL Neph & TL Neph & TL newlv Neph & TL newlv Neph & TL newlv Neph & TL Neph \\
\hline 2 & Case 15 Batch 2 & 53 & TL Neph & TL Neph & TL Neph & TL newly Neph & TL newly Neph & TL newlv Neph & TL Neph \\
\hline 2 & Case 15 Batch 2 & 54 & TL Neph & TL Neph & TL Neph & TL newlv Neph & TL newlv Neph & TL newlv Neph & TL Neph \\
\hline 2 & Case 15 Batch 2 & 55 & TL Neph & TL Neph & TL Neph & TL newlv Neph & TL newlv Neph & TL newlv Neph & TL Neph \\
\hline 2 & Case 15 Batch 2 & 56 & TL Neph & TL Neph & TL Neph & TL newly Neph & TL newly Neph & TL newlv Neph & TL Neph \\
\hline 2 & Case 15 Batch 2 & 57 & TL Neph & TL Neph & TL Neph & TL newlv Neph & TL newlv Neph & TL newlv Neph & TL Neph \\
\hline 2 & Case 15 Batch 2 & 58 & TL Neph & TL Neph & TL Neph & TL newlv Neph & TL newlv Neph & TL newlv Neph & TL Neph \\
\hline 2 & Case 15 Batch 2 & 59 & TL Neph & TL Neph & TL Neph & TL newlv Neph & TL newlv Neph & TL newlv Neph & TL newlv Neph \\
\hline 2 & Case 15 Batch 2 & 60 & TL Neph & TL Neph & TL Neph & TL newlv Neph & TL newlv Neph & TL newlv Neph & TL newlv Neph \\
\hline 2 & Case 15 Blend 1 & 25 & newhv & newhv & newhv & & & & newhv \\
\hline 2 & Case 15 Blend 1 & 26 & newhv & newhv & newhy & & & & \\
\hline 2 & Case 15 Blend 1 & 27 & newhy & newhy & newhy & & & & \\
\hline 2 & Case 15 Blend 1 & 28 & newhv & newhy & newhy & & & & \\
\hline 2 & Case 15 Blend 1 & 29 & newhv & newhv & newhv & & & & \\
\hline 2 & Case 15 Blend 1 & 30 & & newhv & newhv & & & & \\
\hline 2 & Case 15 Blend 1 & 31 & & newhv & & & & & \\
\hline 2 & Case 15 Blend 1 & 32 & & newhv & & & & & \\
\hline 2 & Case 15 Blend 1 & 33 & & newhv & & & & & \\
\hline 2 & Case 15 Blend 1 & 34 & & newhv & & & & & \\
\hline 2 & Case 15 Blend 1 & 35 & & newhv & & & & & \\
\hline 2 & Case 15 Blend 1 & 36 & & & & & & & \\
\hline
\end{tabular}


Exhibit A1. Results for MAR Assessments of the Nominal Stage (part 2)

Constraint not met: neph (nepheline), TL (liquidus temperature), newlv (low viscosity - new model), newhv (high viscosity - new model), and Del GP - durability

\begin{tabular}{|c|c|c|c|c|c|c|c|c|c|}
\hline & & " $\mathrm{Na} 2 \mathrm{O}$ & 0.05 & 0.06 & 0.06 & 0.06 & 0.06 & 0.06 & $\begin{array}{ll}0.07 \\
\end{array}$ \\
\hline Set & Type & Sludge Loading (\%) & $\frac{t 1-1}{t 1}$ & 202 & 432 & P2-3 & P2-4 & $\frac{0.0}{\mathrm{P} 3-2}$ & 460 \\
\hline$\frac{2}{2}$ & Case 15 Blend 1 & 37 & & & & & newlv & & \\
\hline 2 & Case 15 Blend 1 & 38 & & & & & newlv & & \\
\hline 2 & Case 15 Blend 1 & 39 & & $\mathrm{TL}$ & & & newlv & & \\
\hline 2 & Case 15 Blend 1 & 40 & & $\mathrm{TL}$ & & & newlv & & \\
\hline 2 & Case 15 Blend 1 & 41 & $\mathrm{TL}$ & $\mathrm{TL}$ & & & newlv & & \\
\hline 2 & Case 15 Blend 1 & 42 & $\mathrm{TL}$ & $\mathrm{TL}$ & & & TL newlv & & \\
\hline 2 & Case 15 Blend 1 & 43 & $\mathrm{TL}$ & $\mathrm{TL}$ & & $\mathrm{TL}$ & TL newlv & & \\
\hline$\frac{2}{2}$ & Case 15 Blend 1 & 44 & $\mathrm{TL}$ & $\mathrm{TL}$ & $\overline{\mathrm{TL}}$ & $\mathrm{TL}$ & TL newlv & & \\
\hline 2 & Case 15 Blend 1 & 45 & $\mathrm{TL}$ & $\mathrm{TL}$ & $\mathrm{TL}$ & $\mathrm{TL}$ & TL newly & $\mathrm{TL}$ & $\mathrm{TL}$ \\
\hline 2 & Case 15 Blend 1 & 46 & $\mathrm{TL}$ & $\mathrm{TL}$ & $\mathrm{TL}$ & $\mathrm{TL}$ & TL newlv & $\mathrm{TL}$ & $\mathrm{TL}$ \\
\hline 2 & Case 15 Blend 1 & 47 & $\mathrm{TL}$ & $\mathrm{TL}$ & $\mathrm{TL}$ & $\overline{T L}$ & TL newlv Neph & $\mathrm{TL}$ & $\mathrm{TL}$ \\
\hline 2 & Case 15 Blend 1 & 48 & $\mathrm{TL}$ & TL & $\mathrm{TL}$ & TL newlv Neph & TL newlv Neph & $\mathrm{TL}$ & $\mathrm{TL}$ \\
\hline 2 & Case 15 Blend 1 & 49 & $\mathrm{TL}$ & TL Neph & $\mathrm{TL}$ & TL newlv Neph & TL newlv Neph & TL Neph & TL Neph \\
\hline$\frac{2}{2}$ & Case 15 Blend 1 & 50 & TL Neph & TL Neph & TL Neph & TL newlv Neph & TL newlv Neph & TL Neph & TL Neph \\
\hline 2 & Case 15 Blend 1 & 51 & TL Neph & TL Neph & TL Neph & TL newlv Neph & TL newlv Neph & TL Neph & TL Neph \\
\hline 2 & Case 15 Blend 1 & 52 & TL Neph & TL Neph & TL Neph & TL newlv Neph & TL newlv Neph & TL newlv Neph & TL Neph \\
\hline$\frac{2}{2}$ & Case 15 Blend 1 & $\frac{32}{53}$ & TL Neph & TL Neph & TL Neph & TL newlv Neph & TL newlv Neph & TL newlv Neph & TL Neph \\
\hline$\frac{2}{2}$ & Case 15 Blend 1 & 54 & TL Neph & TL Neph & TL Neph & TL newlv Neph & TL newlv Neph & TL newlv Neph & TL Neph \\
\hline 2 & Case 15 Blend 1 & 55 & TL Neph & TL Neph & TL Neph & TL newlv Neph & TL newlv Neph & TL newlv Neph & TL Neph \\
\hline 2 & Case 15 Blend 1 & 56 & TL Neph & TL Neph & TL Neph & TL newlv Neph & TL newlv Neph & TL newlv Neph & TL Neph \\
\hline 2 & Case 15 Blend 1 & 57 & TL Neph & TL Neph & TL Neph & TL newlv Neph & TL newlv Neph & TL newlv Neph & TL Neph \\
\hline 2 & Case 15 Blend 1 & 58 & TL Neph & TL Neph & TL Neph & TL newlv Neph & TL newlv Neph & TL newlv Neph & TL Neph \\
\hline 2 & Case 15 Blend 1 & 59 & TL Neph & TL Neph & TL Neph & TL newlv Neph & TL newlv Neph & TL newlv Neph & TL newlv Neph \\
\hline 2 & Case 15 Blend 1 & 60 & TL newlv Neph & TL Neph & TL newlv Neph & TL newlv Neph & TL newlv Neph & TL newlv Neph & TL newlv Neph \\
\hline 2 & Case 15 Blend 2 & 25 & newhv & newhv & newhy & & & & \\
\hline 2 & Case 15 Blend 2 & 26 & newhv & newhv & newhy & & & & \\
\hline 2 & Case 15 Blend 2 & 27 & newhv & newhv & newhv & & & & \\
\hline$\frac{2}{2}$ & Case 15 Blend 2 & $\frac{21}{28}$ & newhy & newhy & newhy & & & & \\
\hline 2 & Case 15 Blend 2 & 29 & newhv & newhv & newhv & & & & \\
\hline 2 & Case 15 Blend 2 & 30 & & newhv & newhv & & & & \\
\hline 2 & Case 15 Blend 2 & 31 & & newhv & & & & & \\
\hline 2 & Case 15 Blend 2 & 32 & & newhv & & & & & \\
\hline 2 & Case 15 Blend 2 & 33 & & newhy & & & & & \\
\hline 2 & Case 15 Blend 2 & 34 & & newhv & & & & & \\
\hline 2 & Case 15 Blend 2 & 35 & & newhy & & & & & \\
\hline$\frac{2}{2}$ & Case 15 Blend 2 & 36 & & & & & newlv & & \\
\hline 2 & Case 15 Blend 2 & 37 & & & & & newlv & & \\
\hline 2 & Case 15 Blend 2 & 38 & & & & & newlv & & \\
\hline 2 & Case 15 Blend 2 & 39 & & & & & newlv & & \\
\hline 2 & Case 15 Blend 2 & 40 & & & & & newlv & & \\
\hline 2 & Case 15 Blend 2 & 41 & & $\mathrm{TL}$ & & & newlv & & \\
\hline 2 & Case 15 Blend 2 & 42 & $\mathrm{TL}$ & $\mathrm{TL}$ & & & newlv & & \\
\hline$\frac{2}{2}$ & Case 15 Blend 2 & $\frac{72}{43}$ & $\frac{T L}{T L}$ & $\frac{T L}{T L}$ & & & TL newlv Neph & & \\
\hline 2 & Case 15 Blend 2 & 44 & $\mathrm{TL}$ & $\mathrm{TL}$ & & Neph & TL newlv Neph & & \\
\hline 2 & Case 15 Blend 2 & 45 & $\mathrm{TL}$ & $\mathrm{TL}$ & & TL Neph & TL newlv Neph & Neph & Neph \\
\hline 2 & Case 15 Blend 2 & 46 & $\mathrm{TL}$ & TL Neph & TL Neph & TL Neph & TL newlv Neph & Neph & Neph \\
\hline 2 & Case 15 Blend 2 & 47 & TL Neph & TL Neph & TL Neph & TL Neph & TL newlv Neph & TL Neph & TL Neph \\
\hline 2 & Case 15 Blend 2 & 48 & TL Neph & TL Neph & TL Neph & TL newlv Neph & TL newlv Neph & TL Neph & TL Neph \\
\hline 2 & Case 15 Blend 2 & 49 & TL Neph & TL Neph & TL Neph & TL newlv Neph & TL newlv Neph & TL Neph & TL Neph \\
\hline 2 & Case 15 Blend 2 & 50 & TL Neph & TL Neph & TL Neph & TL newlv Neph & TL newlv Neph & TL Neph & TL Neph \\
\hline 2 & Case 15 Blend 2 & 51 & TL Neph & TL Neph & TL Neph & TL newlv Neph & TL newlv Neph & TL newlv Neph & TL Neph \\
\hline 2 & Case 15 Blend 2 & 52 & TL Neph & TL Neph & TL Neph & TL newlv Neph & TL newlv Neph & TL newlv Neph & TL Neph \\
\hline
\end{tabular}


Exhibit A1. Results for MAR Assessments of the Nominal Stage (part 2)

Constraint not met: neph (nepheline), TL (liquidus temperature), newlv (low viscosity - new model), newhv (high viscosity - new model), and Del GP - durability

\begin{tabular}{|c|c|c|c|c|c|c|c|c|c|}
\hline & & $\begin{array}{l}\mathrm{Na} 2 \mathrm{O} \\
\end{array}$ & 0.05 & 0.06 & 0.06 & 0.06 & 0.06 & 0.06 & 0.07 \\
\hline Set & Type & Sludge Loading (\%) & $\mathrm{t} 1-1$ & 202 & 432 & P2-3 & $\mathrm{P} 2-4$ & P3-2 & 460 \\
\hline$\frac{2}{2}$ & Case 15 Blend 2 & 53 & TL Neph & TL Neph & TL Neph & TL newlv Neph & TL newlv Neph & TL newlv Neph & TL Neph \\
\hline 2 & Case 15 Blend 2 & 54 & TL Neph & TL Neph & TL Neph & TL newlv Neph & TL newlv Neph & TL newlv Neph & TL Neph \\
\hline$\frac{2}{2}$ & Case 15 Blend 2 & $\frac{34}{55}$ & TL Neph & TL Neph & TL Neph & TL newlv Neph & TL newlv Neph & TL newlv Neph & TL Neph \\
\hline 2 & Case 15 Blend 2 & 56 & TL Neph & TL Neph & TL Neph & TL newlv Neph & TL newlv Neph & TL newlv Neph & TL Neph \\
\hline 2 & Case 15 Blend 2 & 57 & TL Neph & TL Neph & TL Neph & TL newlv Neph & TL newlv Neph & TL newlv Neph & TL Neph \\
\hline 2 & Case 15 Blend 2 & 58 & TL Neph & TL Neph & TL Neph & TL newlv Neph & TL newlv Neph & TL newlv Neph & TL newlv Neph \\
\hline 2 & Case 15 Blend 2 & 59 & TL Neph & TL Neph & TL Neph & TL newlv Neph & TL newlv Neph & TL newlv Neph & TL newlv Neph \\
\hline 2 & Case 15 Blend 2 & 60 & TL newlv Neph & TL Neph & TL newlv Neph & TL newlv Neph & TL newlv Neph & TL newlv Neph & TL newlv Neph \\
\hline 3 & Case $7 \mathrm{~b}$ Batch 1 & 25 & newhv & newhy & newhv & & & & newhv \\
\hline 3 & Case 7b Batch 1 & 26 & newhv & newhv & newhv & & & & \\
\hline 3 & Case 7b Batch 1 & 27 & newhy & newhy & newhv & & & & \\
\hline 3 & Case $7 \mathrm{~b}$ Batch 1 & 28 & newhv & newhv & newhv & & & & \\
\hline 3 & Case $7 \mathrm{~b}$ Batch 1 & 29 & newhv & newhv & newhv & & & & \\
\hline 3 & Case 7b Batch 1 & 30 & newhy & newhy & newhv & & & & \\
\hline 3 & Case 7b Batch 1 & 31 & & newhv & newhy & & & & \\
\hline 3 & Case 7b Batch 1 & 32 & & newhv & & & & & \\
\hline 3 & Case $7 \mathrm{~b}$ Batch 1 & 33 & & newhv & & & & & \\
\hline$\frac{3}{3}$ & Case 7b Batch 1 & $\frac{34}{34}$ & & newhy & & & & & \\
\hline 3 & Case 7b Batch 1 & 35 & & newhv & & & & & \\
\hline 3 & Case 7b Batch 1 & 36 & & newhy & & & & & \\
\hline 3 & Case 7b Batch 1 & 37 & & & & & & & \\
\hline 3 & Case $7 \mathrm{~b}$ Batch 1 & 38 & & & & & newlv & & \\
\hline 3 & Case 7b Batch 1 & 39 & & & & & newlv & & \\
\hline 3 & Case 7b Batch 1 & 40 & & & & & newlv & & \\
\hline 3 & Case 7b Batch 1 & 41 & & & & & newlv Neph & & \\
\hline 3 & Case 7b Batch 1 & 42 & & & & Neph & newlv Neph & & \\
\hline 3 & Case 7b Batch 1 & 43 & & Neph & & Neph & newlv Neph & Neph & Neph \\
\hline$\frac{3}{3}$ & Case 7b Batch 1 & $\frac{73}{44}$ & Neph & Neph & Neph & Neph & newlv Neph & Neph & Neph \\
\hline 3 & Case 7b Batch 1 & 45 & Neph & Neph & Neph & Neph & newlv Neph & Neph & Neph \\
\hline 3 & Case 7b Batch 1 & 46 & Neph & Neph & Neph & Neph & newlv Neph & Neph & Neph \\
\hline 3 & Case 7b Batch 1 & 47 & Neph & Neph & Neph & Neph & newlv Neph & Neph & Neph \\
\hline 3 & Case 7b Batch 1 & 48 & Neph & TL Neph & Neph & Neph & newlv Neph & Neph & Neph \\
\hline 3 & Case $7 \mathrm{~b}$ Batch 1 & 49 & Neph & TL Neph & Neph & newlv Neph & newlv Neph & Neph & Neph \\
\hline 3 & Case 7b Batch 1 & 50 & TL Neph & TL Neph & Neph & newlv Neph & newlv Neph & Neph & Neph \\
\hline 3 & Case 7b Batch 1 & 51 & TL Neph & TL Neph & Neph & newlv Neph & TL newlv Neph & Neph & Neph \\
\hline 3 & Case 7b Batch 1 & 52 & TL Neph & TL Neph & Neph & TL newlv Neph & TL newlv Neph & Neph & Neph \\
\hline 3 & Case 7b Batch 1 & 53 & TL Neph & TL Neph & Neph & TL newlv Neph & TL newlv Neph & newlv Neph & Neph \\
\hline 3 & Case 7b Batch 1 & 54 & TL Neph & TL Neph & TL Neph & TL newlv Neph & TL newlv Neph & TL newlv Neph & Neph \\
\hline 3 & Case 7b Batch 1 & 55 & TL Neph & TL Neph & TL Neph & TL newlv Neph & TL newlv Neph & TL newlv Neph & TL Neph \\
\hline 3 & Case 7b Batch 1 & 56 & TL Neph & TL Neph & TL Neph & TL newlv Neph & TL newlv Neph & TL newlv Neph & TL Neph \\
\hline 3 & Case 7b Batch 1 & 57 & TL Neph & TL Neph & TL Neph & TL newlv Neph & TL newlv Neph & TL newlv Neph & TL Neph \\
\hline 3 & Case 7b Batch 1 & 58 & TL Neph & TL Neph & TL Neph & TL newlv Neph & TL newlv Neph & TL newlv Neph & TL Neph \\
\hline 3 & Case 7b Batch 1 & 59 & TL Neph & TL Neph & TL Neph & TL newlv Neph & TL newlv Neph & TL newlv Neph & TL Neph \\
\hline 3 & Case $7 \mathrm{~b}$ Batch 1 & 60 & TL Neph & TL Neph & TL Neph & TL newlv Neph & TL newlv Neph & TL newlv Neph & TL newlv Neph \\
\hline 3 & Case $7 \mathrm{~b}$ Batch 2 & 25 & newhv & newhv & newhv & & & & newhv \\
\hline 3 & Case 7b Batch 2 & 26 & newhv & newhv & newhv & & & & newhv \\
\hline 3 & Case 7b Batch 2 & 27 & newhy & newhy & newhv & & & & newhv \\
\hline 3 & Case 7b Batch 2 & 28 & newhv & newhv & newhv & & & & newhy \\
\hline 3 & Case 7b Batch 2 & 29 & newhy & newhv & newhv & & & & newhv \\
\hline 3 & Case 7b Batch 2 & 30 & newhy & newhy & newhy & & & & newhy \\
\hline 3 & Case 7b Batch 2 & 31 & newhy & newhy & newhy & & & & newhy \\
\hline 3 & Case 7b Batch 2 & 32 & newhv & newhv & newhy & & & & newhv \\
\hline
\end{tabular}


Exhibit A1. Results for MAR Assessments of the Nominal Stage (part 2)

Constraint not met: neph (nepheline), TL (liquidus temperature), newlv (low viscosity - new model), newhv (high viscosity - new model), and Del GP - durability

\begin{tabular}{|c|c|c|c|c|c|c|c|c|c|}
\hline & & $\begin{array}{l}\mathrm{Na} 2 \mathrm{O} \\
\end{array}$ & 0.05 & 0.06 & 0.06 & 0.06 & 0.06 & 0.06 & $\begin{array}{ll}0.07 \\
\end{array}$ \\
\hline Set & Type & Sludge Loading (\%) & $\mathrm{t} 1-1$ & 202 & 432 & P2-3 & P2-4 & P3-2 & 460 \\
\hline 3 & Case 7b Batch 2 & 33 & newhv & newhv & newhv & & & & newhv \\
\hline 3 & Case 7b Batch 2 & 34 & newhy & newhv & newhy & & & & newhv \\
\hline 3 & Case 7b Batch 2 & 35 & newhv & newhy & newhv & & & & newhv \\
\hline 3 & Case 7b Batch 2 & 36 & newhy & newhy & newhv & & & & newhv \\
\hline 3 & Case 7b Batch 2 & 37 & newhy & newhy & newhv & & & & \\
\hline 3 & Case $7 \mathrm{~b}$ Batch 2 & 38 & newhv & newhv & newhv & & & & \\
\hline$\frac{5}{3}$ & Case 7b Batch 2 & 39 & newhy & newhy & newhv & & & & \\
\hline 3 & Case 7b Batch 2 & 40 & newhv & TL newhy & newhy & & & & \\
\hline 3 & Case $7 \mathrm{~b}$ Batch 2 & 41 & TL newhy & TL newhy & newhv & & & & \\
\hline 3 & Case 7b Batch 2 & 42 & TL newhy & TL newhy & newhv & & TL Neph & & \\
\hline 3 & Case 7b Batch 2 & $\frac{23}{43}$ & $\mathrm{TL}$ & TL newhy & newhv & TL Neph & TL Neph & & \\
\hline 3 & Case 7b Batch 2 & 44 & $\mathrm{TL}$ & TL newhy & & TL Neph & TL Neph & Neph & Neph \\
\hline 3 & Case $7 \mathrm{~b}$ Batch 2 & 45 & TL Neph & TL newhv Neph & TL Neph & TL Neph & TL Neph & Neph & Neph \\
\hline 3 & Case 7b Batch 2 & 46 & TL Neph & TL newhv Neph & TL Neph & TL Neph & TL Neph & TL Neph & TL Neph \\
\hline 3 & Case $7 \mathrm{~b}$ Batch 2 & 47 & TL Neph & TL newhv Neph & TL Neph & TL Neph & TL Neph & TL Neph & TL Neph \\
\hline 3 & Case 7b Batch 2 & 48 & TL Neph & TL newhv Neph & TL Neph & TL Neph & TL Neph & TL Neph & TL Neph \\
\hline 3 & Case $7 \mathrm{~b}$ Batch 2 & 49 & TL Neph & TL newhy Neph & TL Neph & TL Neph & TL Neph & TL Neph & TL Neph \\
\hline$\frac{3}{3}$ & Case 7b Batch 2 & 50 & TL Neph & TL Neph & TL Neph & TL Neph & TL Neph & TL Neph & TL Neph \\
\hline 3 & Case 7b Batch 2 & 51 & TL Neph & TL Neph & TL Neph & TL Neph & TL newlv Neph & TL Neph & TL Neph \\
\hline 3 & Case 7b Batch 2 & 52 & TL Neph & TL Neph & TL Neph & TL Neph & TL newlv Neph & TL Neph & TL Neph \\
\hline 3 & Case 7b Batch 2 & 53 & TL Neph & TL Neph & TL Neph & TL Neph & TL newlv Neph & TL Neph & TL Neph \\
\hline 3 & Case $7 \mathrm{~b}$ Batch 2 & 54 & TL Neph & TL Neph & TL Neph & TL Neph & TL newlv Neph & TL Neph & TL Neph \\
\hline 3 & Case 7b Batch 2 & 55 & TL Neph & TL Neph & TL Neph & TL Neph & TL newlv Neph & TL Neph & TL Neph \\
\hline 3 & Case 7b Batch 2 & 56 & TL Neph & TL Neph & TL Neph & TL Neph & TL newlv Neph & TL Neph & TL Neph \\
\hline 3 & Case 7b Batch 2 & 57 & TL Neph & TL Neph & TL Neph & TL Neph & TL newlv Neph & TL Neph & TL Neph \\
\hline 3 & Case 7b Batch 2 & 58 & TL Neph & TL Neph & TL Neph & TL Neph & TL newlv Neph & TL Neph & TL Neph \\
\hline 3 & Case 7b Batch 2 & 59 & TL Neph & TL Neph & TL Neph & TL Neph & TL newlv Neph & TL Neph & TL Neph \\
\hline$\frac{3}{3}$ & Case 7b Batch 2 & 60 & TL Neph & TL Neph & TL Neph & TL Neph & TL newlv Neph & TL Neph & TL Neph \\
\hline 3 & Case $7 \mathrm{~b}$ Blend 1 & 25 & & newhv & & & & & \\
\hline 3 & Case $7 \mathrm{~b}$ Blend 1 & 26 & & newhy & & & & & \\
\hline 3 & Case $7 \mathrm{~b}$ Blend 1 & 27 & & newhv & & & & & \\
\hline 3 & Case $7 \mathrm{~b}$ Blend 1 & 28 & & & & & & & \\
\hline 3 & Case $7 \mathrm{~b}$ Blend 1 & 29 & & & & & newlv & & \\
\hline 3 & Case $7 \mathrm{~b}$ Blend 1 & 30 & & & & & newlv & & \\
\hline 3 & Case $7 \mathrm{~b}$ Blend 1 & 31 & & & & & newlv & & \\
\hline 3 & Case $7 \mathrm{~b}$ Blend 1 & 32 & & & & & newlv & & \\
\hline 3 & Case $7 \mathrm{~b}$ Blend 1 & 33 & & & & & newlv & & \\
\hline 3 & Case $7 \mathrm{~b}$ Blend 1 & 34 & & & & & newlv & & \\
\hline 3 & Case $7 \mathrm{~b}$ Blend 1 & 35 & & & & & newlv & & \\
\hline 3 & Case $7 \mathrm{~b}$ Blend 1 & 36 & & & & & newlv & & \\
\hline 3 & Case $7 \mathrm{~b}$ Blend 1 & 37 & & & & & newlv & & \\
\hline 3 & Case $7 \mathrm{~b}$ Blend 1 & 38 & & & & & newlv & & \\
\hline 3 & Case $7 \mathrm{~b}$ Blend 1 & 39 & & & & newlv & newlv & & \\
\hline 3 & Case $7 \mathrm{~b}$ Blend 1 & 40 & & & & newlv & newlv & & \\
\hline 3 & Case $7 \mathrm{~b}$ Blend 1 & 41 & & & & newlv & newlv & & \\
\hline$\frac{3}{3}$ & Case $7 \mathrm{~b}$ Blend 1 & $\frac{71}{42}$ & & $\mathrm{TL}$ & & newlv & newlv & newlv & \\
\hline 3 & Case $7 \mathrm{~b}$ Blend 1 & 43 & & $\mathrm{TL}$ & & newlv & newlv & newlv & \\
\hline 3 & Case $7 \mathrm{~b}$ Blend 1 & 44 & $\mathrm{TL}$ & $\mathrm{TL}$ & & newlv & newlv & newlv & \\
\hline 3 & Case $7 \mathrm{~b}$ Blend 1 & 45 & $\mathrm{TL}$ & $\mathrm{TL}$ & & newlv & TL newlv & newlv & \\
\hline 3 & Case $7 \mathrm{~b}$ Blend 1 & 46 & $\mathrm{TL}$ & $\mathrm{TL}$ & & TL newlv & TL newlv & newlv & \\
\hline 3 & Case $7 \mathrm{~b}$ Blend 1 & 47 & $\mathrm{TL}$ & $\mathrm{TL}$ & $\mathrm{TL}$ & TL newlv & TL newlv Neph & newlv & \\
\hline 3 & Case $7 \mathrm{~b}$ Blend 1 & 48 & $\mathrm{TL}$ & $\mathrm{TL}$ & $\mathrm{TL}$ & TL newlv Neph & TL newlv Neph & TL newlv & $\mathrm{TL}$ \\
\hline
\end{tabular}


Exhibit A1. Results for MAR Assessments of the Nominal Stage (part 2)

Constraint not met: neph (nepheline), TL (liquidus temperature), newlv (low viscosity - new model), newhv (high viscosity - new model), and Del GP - durability

\begin{tabular}{|c|c|c|c|c|c|c|c|c|c|}
\hline & & $\mathrm{Na} 2 \mathrm{O}$ & 0.05 & 0.06 & 0.06 & 0.06 & 0.06 & 0.06 & 0.07 \\
\hline Set & Type & Sludge Loading (\%) & $\mathrm{t} 1-1$ & 202 & 432 & P2-3 & P2-4 & P3-2 & 460 \\
\hline 3 & Case $7 \mathrm{~b}$ Blend 1 & 49 & $\mathrm{TL}$ & $\mathrm{TL}$ & $\mathrm{TL}$ & TL newlv Neph & TL newlv Neph & TL newlv Neph & TL newlv Neph \\
\hline 3 & Case $7 \mathrm{~b}$ Blend 1 & 50 & TL Neph & TL Neph & TL Neph & TL newlv Neph & TL newly Neph & TL newlv Neph & TL newlv Neph \\
\hline 3 & Case $7 \mathrm{~b}$ Blend 1 & 51 & TL newlv Neph & TL Neph & TL newlv Neph & TL newlv Neph & TL newlv Neph & TL newlv Neph & TL newlv Neph \\
\hline 3 & Case $7 \mathrm{~b}$ Blend 1 & 52 & TL newlv Neph & TL Neph & TL newlv Neph & TL newlv Neph & TL newlv Neph & TL newlv Neph & TL newlv Neph \\
\hline 3 & Case $7 \mathrm{~b}$ Blend 1 & 53 & TL newlv Neph & TL newlv Neph & TL newlv Neph & TL newlv Neph & TL newlv Neph & TL newlv Neph & TL newlv Neph \\
\hline 3 & Case $7 \mathrm{~b}$ Blend 1 & 54 & TL newlv Neph & TL newlv Neph & TL newlv Neph & TL newlv Neph & TL newlv Neph & TL newlv Neph & TL newlv Neph \\
\hline 3 & Case $7 \mathrm{~b}$ Blend 1 & 55 & TL newlv Neph & TL newlv Neph & TL newlv Neph & TL newlv Neph & TL newlv Neph & TL newlv Neph & TL newlv Neph \\
\hline 3 & Case $7 \mathrm{~b}$ Blend 1 & 56 & TL newlv Neph & TL newlv Neph & TL newlv Neph & TL newlv Neph & TL newlv Neph & TL newlv Neph & TL newlv Neph \\
\hline 3 & Case $7 \mathrm{~b}$ Blend 1 & 57 & TL newlv Neph & TL newlv Neph & TL newlv Neph & TL newly Neph & TL newlv Neph & TL newlv Neph & TL newlv Neph \\
\hline 3 & Case $7 \mathrm{~b}$ Blend 1 & 58 & TL newlv Neph & TL newlv Neph & TL newlv Neph & TL newly Neph & TL newlv Neph & TL newlv Neph & TL newlv Neph \\
\hline 3 & Case $7 \mathrm{~b}$ Blend 1 & 59 & TL newlv Neph & TL newlv Neph & TL newlv Neph & TL newlv Neph & TL newlv Neph & TL newlv Neph & TL newlv Neph \\
\hline 3 & Case $7 \mathrm{~b}$ Blend 1 & 60 & TL newlv Neph & TL newlv Neph & TL newlv Neph & TL newlv Neph & TL newlv Neph & TL newlv Neph & TL newlv Neph \\
\hline 3 & Case $7 \mathrm{~b}$ Blend 2 & 25 & newhv & newhv & newhy & & & & newhv \\
\hline 3 & Case $7 \mathrm{~b}$ Blend 2 & 26 & newhv & newhv & newhv & & & & newhv \\
\hline 3 & Case $7 \mathrm{~b}$ Blend 2 & 27 & newhv & newhv & newhy & & & & newhy \\
\hline 3 & Case $7 \mathrm{~b}$ Blend 2 & 28 & newhv & newhv & newhv & & & & newhv \\
\hline 3 & Case $7 \mathrm{~b}$ Blend 2 & 29 & newhv & newhv & newhv & & & & newhv \\
\hline 3 & Case $7 \mathrm{~b}$ Blend 2 & 30 & newhy & newhy & newhv & & & & \\
\hline 3 & Case $7 \mathrm{~b}$ Blend 2 & 31 & newhv & newhv & newhv & & & & \\
\hline 3 & Case $7 \mathrm{~b}$ Blend 2 & 32 & newhy & newhv & newhy & & & & \\
\hline 3 & Case $7 \mathrm{~b}$ Blend 2 & 33 & newhv & newhv & newhv & & & & \\
\hline 3 & Case $7 \mathrm{~b}$ Blend 2 & 34 & newhy & newhy & newhy & & & & \\
\hline 3 & Case $7 \mathrm{~b}$ Blend 2 & 35 & & newhv & newhy & & & & \\
\hline 3 & Case $7 \mathrm{~b}$ Blend 2 & 36 & & newhy & & & & & \\
\hline 3 & Case $7 \mathrm{~b}$ Blend 2 & 37 & & newhv & & & & & \\
\hline 3 & Case $7 \mathrm{~b}$ Blend 2 & 38 & & newhv & & & & & \\
\hline 3 & Case $7 \mathrm{~b}$ Blend 2 & 39 & & newhv & & & & & \\
\hline 3 & Case $7 \mathrm{~b}$ Blend 2 & 40 & & newhv & & & & & \\
\hline 3 & Case $7 \mathrm{~b}$ Blend 2 & 41 & & $\mathrm{TL}$ & & & & & \\
\hline 3 & Case $7 \mathrm{~b}$ Blend 2 & 42 & $\mathrm{TL}$ & $\mathrm{TL}$ & & & newlv & & \\
\hline 3 & Case $7 \mathrm{~b}$ Blend 2 & 43 & $\mathrm{TL}$ & $\mathrm{TL}$ & & & TL newlv Neph & & \\
\hline 3 & Case $7 \mathrm{~b}$ Blend 2 & 44 & TL & TL & & TL Neph & TL newlv Neph & & \\
\hline 3 & Case $7 \mathrm{~b}$ Blend 2 & 45 & $\mathrm{TL}$ & $\mathrm{TL}$ & & TL Neph & TL newlv Neph & Neph & Neph \\
\hline 3 & Case $7 \mathrm{~b}$ Blend 2 & 46 & TL Neph & TL Neph & TL Neph & TL Neph & TL newlv Neph & Neph & Neph \\
\hline 3 & Case $7 \mathrm{~b}$ Blend 2 & 47 & TL Neph & TL Neph & TL Neph & TL Neph & TL newly Neph & TL Neph & TL Neph \\
\hline 3 & Case $7 \mathrm{~b}$ Blend 2 & 48 & TL Neph & TL Neph & TL Neph & TL Neph & TL newlv Neph & TL Neph & TL Neph \\
\hline 3 & Case $7 \mathrm{~b}$ Blend 2 & 49 & TL Neph & TL Neph & TL Neph & TL Neph & TL newlv Neph & TL Neph & TL Neph \\
\hline 3 & Case $7 \mathrm{~b}$ Blend 2 & 50 & TL Neph & TL Neph & TL Neph & TL Neph & TL newlv Neph & TL Neph & TL Neph \\
\hline 3 & Case $7 \mathrm{~b}$ Blend 2 & 51 & TL Neph & TL Neph & TL Neph & TL Neph & TL newlv Neph & TL Neph & TL Neph \\
\hline 3 & Case $7 \mathrm{~b}$ Blend 2 & 52 & TL Neph & TL Neph & TL Neph & TL Neph & TL newlv Neph & TL Neph & TL Neph \\
\hline 3 & Case $7 \mathrm{~b}$ Blend 2 & 53 & TL Neph & TL Neph & TL Neph & TL Neph & TL newlv Neph & TL Neph & TL Neph \\
\hline 3 & Case $7 \mathrm{~b}$ Blend 2 & 54 & TL Neph & TL Neph & TL Neph & TL newlv Neph & TL newlv Neph & TL Neph & TL Neph \\
\hline 3 & Case $7 \mathrm{~b}$ Blend 2 & 55 & TL Neph & TL Neph & TL Neph & TL newlv Neph & TL newlv Neph & TL Neph & TL Neph \\
\hline 3 & Case $7 \mathrm{~b}$ Blend 2 & 56 & TL Neph & TL Neph & TL Neph & TL newlv Neph & TL newlv Neph & TL Neph & TL Neph \\
\hline 3 & Case $7 \mathrm{~b}$ Blend 2 & 57 & TL Neph & TL Neph & TL Neph & TL newlv Neph & TL newlv Neph & TL newlv Neph & TL Neph \\
\hline 3 & Case $7 \mathrm{~b}$ Blend 2 & 58 & TL Neph & TL Neph & TL Neph & TL newlv Neph & TL newlv Neph & TL newlv Neph & TL Neph \\
\hline 3 & Case $7 \mathrm{~b}$ Blend 2 & 59 & TL Neph & TL Neph & TL Neph & TL newlv Neph & TL newlv Neph & TL newlv Neph & TL Neph \\
\hline 3 & Case $7 \mathrm{~b}$ Blend 2 & 60 & TL Neph & TL Neph & TL Neph & TL newlv Neph & TL newlv Neph & TL newlv Neph & TL Neph \\
\hline
\end{tabular}


Exhibit A1. Results for MAR Assessments of the Nominal Stage (part 3)

Constraint not met: neph (nepheline), TL (liquidus temperature), newlv (low viscosity - new model), newhv (high viscosity - new model), and Del GP - durability

\begin{tabular}{|c|c|c|c|c|c|c|c|c|c|}
\hline & & Na2O & $\begin{array}{ll}0.07 \\
\end{array}$ & $\begin{array}{ll}0.07 \\
\end{array}$ & 0.07 & 0.08 & 0.09 & 0.1 & 0.11 \\
\hline Set & $\begin{array}{l}\text { Type } \\
\end{array}$ & Sludge Loading (\%) & P2-5 & P3-3 & P3-4 & 418 & 426 & 425 & 200 \\
\hline 1 & Case 15B - Batch 1 & 25 & & & & newhv & newhv & & newhv \\
\hline 1 & Case 15B - Batch 1 & 26 & & & & newhy & newhv & & newhy \\
\hline 1 & Case 15B - Batch 1 & 27 & & & & newhy & newhy & & newhv \\
\hline 1 & Case 15B - Batch 1 & 28 & & & & newhv & newhy & & newhv \\
\hline$\frac{1}{1}$ & Case 15B - Batch 1 & 29 & & & & newhy & newhy & & newhy \\
\hline 1 & Case 15B - Batch 1 & 30 & & & & newhv & newhv & & newhv \\
\hline 1 & Case 15B - Batch 1 & 31 & & & & newhy & newhv & & newhv \\
\hline 1 & Case 15B - Batch 1 & 32 & & & & newhv & newhy & & newhy \\
\hline 1 & Case 15B - Batch 1 & 33 & & & & newhv & newhv & & newhy \\
\hline 1 & Case 15B - Batch 1 & 34 & & & & newhv & newhv & & newhv \\
\hline 1 & Case 15B - Batch 1 & 35 & & & & newhv & newhy & & newhv Neph \\
\hline$\frac{1}{1}$ & Case 15B - Batch 1 & 36 & & & & newhy & newhy & & newhv Neph \\
\hline 1 & Case 15B - Batch 1 & 37 & & & & newhv & newhv & Neph & newhv Neph \\
\hline 1 & Case 15B - Batch 1 & 38 & Neph & & & newhy & newhv Neph & Neph & newhv Neph \\
\hline$\frac{1}{1}$ & Case 15B - Batch 1 & $\frac{30}{39}$ & Neph & Neph & & newhy & newhv Neph & Neph & newhv Neph \\
\hline$\frac{1}{1}$ & Case 15B - Batch 1 & 40 & Neph & Neph & Neph & newhy Neph & newhv Neph & Neph & newhv Neph \\
\hline 1 & Case 15B - Batch 1 & 41 & Neph & Neph & Neph & newhv Neph & newhv Neph & Neph & newhv Neph \\
\hline 1 & Case 15B - Batch 1 & 42 & Neph & Neph & Neph & newhv Neph & newhv Neph & Neph & newhv Neph \\
\hline 1 & Case 15B - Batch 1 & 43 & Neph & Neph & Neph & newhv Neph & newhv Neph & Neph & newhv Neph \\
\hline 1 & Case 15B - Batch 1 & 44 & Neph & Neph & Neph & newhv Neph & newhv Neph & Neph & newhv Neph \\
\hline 1 & Case 15B - Batch 1 & 45 & Neph & Neph & Neph & newhy Neph & newhv Neph & Neph & newhv Neph \\
\hline 1 & Case 15B - Batch 1 & 46 & Neph & Neph & Neph & newhv Neph & newhv Neph & Neph & newhv Neph \\
\hline$\frac{1}{1}$ & Case 15B - Batch 1 & $\frac{70}{47}$ & Neph & Neph & Neph & newhy Neph & newhv Neph & Neph & newhv Neph \\
\hline 1 & Case 15B - Batch 1 & 48 & Neph & Neph & Neph & newhv Neph & newhv Neph & Neph & newhv Neph \\
\hline 1 & Case 15B - Batch 1 & 49 & Neph & Neph & Neph & newhy Neph & newhy Neph & Neph & newhv Neph \\
\hline$\frac{1}{1}$ & Case 15B - Batch 1 & 50 & Neph & Neph & Neph & newhy Neph & newhv Neph & Neph & newhv Neph \\
\hline 1 & Case 15B - Batch 1 & 51 & Neph & Neph & Neph & newhv Neph & newhv Neph & Neph & newhv Neph \\
\hline 1 & $\begin{array}{l}\text { Case 15B - Batch } 1 \\
\end{array}$ & 52 & Neph & Neph & Neph & newhv Neph & Neph & Neph & newhv Neph \\
\hline 1 & Case 15B - Batch 1 & 53 & Neph & Neph & Neph & newhv Neph & Neph & Neph & newhv Neph \\
\hline 1 & Case 15B - Batch 1 & 54 & Neph & Neph & Neph & newhy Neph & Neph & Neph & newhv Neph \\
\hline$\frac{1}{1}$ & Case 15B - Batch 1 & 55 & Neph & Neph & Neph & newhv Neph & Neph & Neph & newhv Neph \\
\hline 1 & Case 15B - Batch 1 & 56 & Neph & Neph & Neph & newhy Neph & Neph & Neph & newhv Neph \\
\hline 1 & Case 15B - Batch 1 & 57 & TL Neph & Neph & Neph & newhv Neph & Neph & Neph & Neph \\
\hline 1 & Case 15B - Batch 1 & 58 & TL Neph & Neph & Neph & newhy Neph & Neph & Neph & Neph \\
\hline$\frac{1}{1}$ & Case 15B - Batch 1 & $\frac{30}{59}$ & TL Neph & Neph & Neph & newhy Neph & Neph & Neph & TL Neph \\
\hline 1 & Case 15B - Batch 1 & 60 & TL Neph & Neph & Neph & newhv Neph & Neph & Neph & TL Neph \\
\hline 1 & Case 15B - Batch 2 & 25 & & & & newhy & & & newhv \\
\hline 1 & Case 15B - Batch 2 & 26 & & & & newhy & & & \\
\hline 1 & Case 15B - Batch 2 & 27 & & & & newhv & & & \\
\hline 1 & Case 15B - Batch 2 & 28 & & & & newhv & & & \\
\hline 1 & Case 15B - Batch 2 & 29 & & & & newhv & & & \\
\hline 1 & Case 15B - Batch 2 & 30 & & & & newhv & & & \\
\hline 1 & Case 15B - Batch 2 & 31 & & & & newhy & & & \\
\hline 1 & Case 15B - Batch 2 & 32 & & & & newhv & & & \\
\hline$\frac{1}{1}$ & Case 15B - Batch 2 & 33 & & & & & & & \\
\hline 1 & Case 15B - Batch 2 & 34 & & & & & & & \\
\hline 1 & Case 15B - Batch 2 & 35 & & & & & & & \\
\hline$\frac{1}{1}$ & Case 15B - Batch 2 & 36 & & & & & & & \\
\hline 1 & Case 15B - Batch 2 & 37 & & & & & & & \\
\hline 1 & Case 15B - Batch 2 & 38 & & & & & & & \\
\hline$\frac{1}{1}$ & Case 15B - Batch 2 & 39 & $\mathrm{TL}$ & & & & & & Neph \\
\hline
\end{tabular}


Exhibit A1. Results for MAR Assessments of the Nominal Stage (part 3)

Constraint not met: neph (nepheline), TL (liquidus temperature), newlv (low viscosity - new model), newhv (high viscosity - new model), and Del GP - durability

\begin{tabular}{|c|c|c|c|c|c|c|c|c|c|}
\hline & & $\mathrm{Na} 2 \mathrm{O}$ & 0.07 & 0.07 & 0.07 & 0.08 & 0.09 & 0.1 & 0.11 \\
\hline Set & $\begin{array}{c}\text { Type } \\
\end{array}$ & Sludge Loading (\%) & $\mathrm{P2} 2-5$ & P3-3 & $\begin{array}{l}\text { P3-4 } \\
\end{array}$ & 418 & 426 & 425 & 200 \\
\hline 1 & Case 15B - Batch 2 & 40 & $\mathrm{TL}$ & & & & & & TL Neph \\
\hline 1 & Case 15B - Batch 2 & 41 & $\mathrm{TL}$ & & & & & & TL Neph \\
\hline 1 & Case 15B - Batch 2 & 42 & $\begin{array}{l}\text { TL Neph } \\
\end{array}$ & & & & & Neph & TL Neph \\
\hline 1 & Case 15B - Batch 2 & 43 & TL Neph & $\mathrm{TL}$ & & & Neph & Neph & TL Neph \\
\hline 1 & Case 15B - Batch 2 & 44 & TL Neph & TL Neph & TL Neph & TL Neph & TL Neph & Neph & TL Neph \\
\hline 1 & Case 15B - Batch 2 & 45 & TL Neph & TL Neph & TL Neph & TL Neph & TL Neph & TL Neph & TL Neph \\
\hline 1 & Case 15B - Batch 2 & 46 & TL Neph & TL Neph & TL Neph & TL Neph & TL Neph & TL Neph & TL Neph \\
\hline 1 & Case 15B - Batch 2 & 47 & TL Neph & TL Neph & TL Neph & TL Neph & TL Neph & TL Neph & TL Neph \\
\hline 1 & Case 15B - Batch 2 & 48 & TL Neph & TL Neph & TL Neph & TL Neph & TL Neph & TL Neph & TL Neph \\
\hline 1 & Case 15B - Batch 2 & 49 & TL Neph & TL Neph & TL Neph & TL Neph & TL Neph & TL Neph & TL Neph \\
\hline 1 & Case 15B - Batch 2 & 50 & TL Neph & TL Neph & TL Neph & TL Neph & TL Neph & TL Neph & TL Neph \\
\hline 1 & Case 15B - Batch 2 & 51 & TL Neph & TL Neph & TL Neph & TL Neph & TL Neph & TL Neph & TL Neph \\
\hline 1 & Case 15B - Batch 2 & 52 & TL Neph & TL Neph & TL Neph & TL Neph & TL Neph & TL Neph & TL Neph \\
\hline 1 & Case 15B - Batch 2 & 53 & TL Neph & TL Neph & TL Neph & TL Neph & TL Neph & TL Neph & TL Neph \\
\hline 1 & Case 15B - Batch 2 & 54 & TL Neph & TL Neph & TL Neph & TL Neph & TL Neph & TL Neph & TL Neph \\
\hline 1 & Case 15B - Batch 2 & 55 & TL Neph & TL Neph & TL Neph & TL Neph & TL Neph & TL Neph & TL Neph \\
\hline 1 & Case 15B - Batch 2 & 56 & TL Neph & TL Neph & TL Neph & TL Neph & TL Neph & TL Neph & TL Neph \\
\hline 1 & Case 15B - Batch 2 & 57 & TL Neph & TL Neph & TL Neph & TL Neph & TL Neph & TL Neph & TL Neph \\
\hline 1 & Case 15B - Batch 2 & 58 & TL newlv Neph & TL Neph & TL Neph & TL Neph & TL Neph & TL Neph & TL Neph \\
\hline 1 & Case 15B - Batch 2 & 59 & TL newlv Neph & TL Neph & TL Neph & TL Neph & TL Neph & TL Neph & TL Neph \\
\hline 1 & Case 15B - Batch 2 & 60 & TL newlv Neph & TL Neph & TL Neph & TL Neph & TL Neph & TL Neph & TL Neph \\
\hline 1 & $\begin{array}{l}\text { Case 15B - Blend } 1 \\
\end{array}$ & 25 & & & & & & & \\
\hline 1 & $\begin{array}{l}\text { Case 15B - Blend } 1 \\
\end{array}$ & 26 & & & & & & & \\
\hline 1 & $\begin{array}{l}\text { Case 15B - Blend } 1 \\
\end{array}$ & 27 & & & & & & & \\
\hline 1 & Case 15B - Blend 1 & 28 & & & & & & & \\
\hline 1 & Case 15B - Blend 1 & 29 & & & & & & & \\
\hline 1 & Case $15 \mathrm{~B}-$ Blend 1 & 30 & & & & & & & \\
\hline 1 & $\begin{array}{l}\text { Case 15B - Blend } 1 \\
\end{array}$ & 31 & newlv & & & & & & \\
\hline 1 & Case 15B - Blend 1 & 32 & newlv & & & & & & \\
\hline 1 & Case 15B - Blend 1 & 33 & newlv & & & & & & \\
\hline 1 & Case 15B - Blend 1 & 34 & newlv & & & & & & \\
\hline 1 & Case $15 \mathrm{~B}-$ Blend 1 & 35 & newlv & & & & & & \\
\hline 1 & Case 15B - Blend 1 & 36 & newlv & & & & & & \\
\hline 1 & Case 15B - Blend 1 & 37 & newlv & & & & & & \\
\hline 1 & $\begin{array}{l}\text { Case 15B - Blend } 1 \\
\end{array}$ & 38 & newlv & & & & & & \\
\hline 1 & Case 15B - Blend 1 & 39 & newlv & newlv & & & & & \\
\hline 1 & Case 15B - Blend 1 & 40 & newlv & newlv & & & & & \\
\hline 1 & Case 15B - Blend 1 & 41 & newlv & newlv & newlv & & & & \\
\hline 1 & $\begin{array}{l}\text { Case 15B - Blend } 1 \\
\end{array}$ & 42 & newlv & $\begin{array}{l}\text { newlv } \\
\end{array}$ & newlv & & & newlv & \\
\hline 1 & Case 15B - Blend 1 & 43 & newlv & newlv & newlv & & & newlv & newlv \\
\hline 1 & Case 15B - Blend 1 & 44 & TL newlv & $\frac{\text { newlv }}{\text { nelv }}$ & newlv & & newlv & newlv & TL newlv \\
\hline 1 & $\begin{array}{l}\text { Case 15B - Blend } 1 \\
\end{array}$ & 45 & TL newlv & newlv & newlv & & newlv & newlv & TL newlv Neph \\
\hline 1 & Case 15B - Blend 1 & 46 & TL newlv & newlv & newlv & & $\begin{array}{ll}\text { newlv } \\
\end{array}$ & newlv & TL newlv Neph \\
\hline 1 & Case 15B - Blend 1 & 47 & TL newlv & newlv & newlv & newlv & newlv & newlv & TL newlv Neph \\
\hline 1 & Case 15B - Blend 1 & 48 & TL newlv Neph & TL newlv & newlv & TL newlv & newlv & newlv Neph & TL newlv Neph \\
\hline 1 & $\begin{array}{l}\text { Case } 15 \mathrm{~B} \text { - Blend } 1 \\
\end{array}$ & 49 & TL newlv Neph & TL newlv & TL newlv & TL newlv & TL newlv Neph & newlv Neph & TL newlv Neph \\
\hline 1 & Case 15B - Blend 1 & 50 & TL newlv Neph & TL newlv Neph & TL newlv Neph & TL newlv Neph & TL newlv Neph & TL newlv Neph & TL newlv Neph \\
\hline 1 & $\begin{array}{l}\text { Case 15B - Blend } 1 \\
\end{array}$ & 51 & TL newlv Neph & TL newlv Neph & TL newlv Neph & TL newlv Neph & TL newlv Neph & TL newlv Neph & TL newlv Neph \\
\hline 1 & Case 15B - Blend 1 & 52 & TL newlv Neph & TL newlv Neph & TL newlv Neph & TL newlv Neph & TL newlv Neph & TL newlv Neph & TL newlv Neph \\
\hline 1 & $\begin{array}{l}\text { Case 15B - Blend } 1 \\
\end{array}$ & 53 & TL newlv Neph & TL newlv Neph & TL newlv Neph & TL newlv Neph & TL newlv Neph & TL newlv Neph & TL newlv Neph \\
\hline 1 & Case 15B - Blend 1 & 54 & TL newlv Neph & TL newlv Neph & TL newlv Neph & TL newlv Neph & TL newlv Neph & TL newlv Neph & TL newlv Neph \\
\hline
\end{tabular}


Exhibit A1. Results for MAR Assessments of the Nominal Stage (part 3)

Constraint not met: neph (nepheline), TL (liquidus temperature), newlv (low viscosity - new model), newhv (high viscosity - new model), and Del GP - durability

\begin{tabular}{|c|c|c|c|c|c|c|c|c|c|}
\hline & & $\mathrm{Na} 2 \mathrm{O}$ & 0.07 & 0.07 & 0.07 & 0.08 & 0.09 & 0.1 & 0.11 \\
\hline Set & $\begin{array}{c}\text { Type } \\
\end{array}$ & Sludge Loading (\%) & P2-5 & P3-3 & P3-4 & 418 & 426 & 425 & 200 \\
\hline 1 & Case 15B - Blend 1 & 55 & TL newlv Neph & TL newlv Neph & TL newlv Neph & TL newlv Neph & TL newlv Neph & TL newlv Neph & TL newlv Neph \\
\hline 1 & Case 15B - Blend 1 & 56 & TL newlv Neph & TL newlv Neph & TL newlv Neph & TL newlv Neph & TL newlv Neph & TL newlv Neph & TL newlv Neph \\
\hline 1 & Case 15B - Blend 1 & 57 & TL newlv Neph & TL newlv Neph & TL newlv Neph & TL newlv Neph & TL newlv Neph & TL newlv Neph & TL newlv Neph \\
\hline 1 & Case 15B - Blend 1 & 58 & TL newlv Neph & TL newlv Neph & TL newlv Neph & TL newlv Neph & TL newlv Neph & TL newlv Neph & TL newlv Neph \\
\hline 1 & Case 15B - Blend 1 & 59 & TL newlv Neph & TL newlv Neph & TL newlv Neph & TL newlv Neph & TL newlv Neph & TL newlv Neph & TL newlv Neph \\
\hline 1 & Case 15B - Blend 1 & 60 & TL newlv Neph & TL newlv Neph & TL newlv Neph & TL newlv Neph & TL newlv Neph & TL newlv Neph & TL newlv Neph \\
\hline 1 & Case $15 \mathrm{~B}$ - Blend 2 & 25 & & & & & & & \\
\hline 1 & Case 15B - Blend 2 & 26 & & & & & & & \\
\hline 1 & Case 15B - Blend 2 & 27 & & & & & & & \\
\hline 1 & Case 15B - Blend 2 & 28 & & & & & & & \\
\hline 1 & Case $15 \mathrm{~B}$ - Blend 2 & 29 & & & & & & & \\
\hline 1 & Case 15B - Blend 2 & 30 & & & & & & & \\
\hline 1 & Case 15B - Blend 2 & 31 & & & & & & & \\
\hline 1 & Case 15B - Blend 2 & 32 & & & & & & & \\
\hline 1 & Case 15B - Blend 2 & 33 & & & & & & & \\
\hline 1 & Case 15B - Blend 2 & 34 & & & & & & & \\
\hline 1 & Case 15B - Blend 2 & 35 & & & & & & & \\
\hline 1 & Case 15B - Blend 2 & 36 & & & & & & & \\
\hline 1 & Case 15B - Blend 2 & 37 & & & & & & & \\
\hline 1 & Case 15B - Blend 2 & 38 & & & & & & & \\
\hline 1 & Case 15B - Blend 2 & 39 & & & & & & & \\
\hline 1 & Case $15 \mathrm{~B}$ - Blend 2 & 40 & $\mathrm{TL}$ & & & & & & Neph \\
\hline 1 & Case 15B - Blend 2 & 41 & TL & & & & & & TL Neph \\
\hline 1 & Case 15B - Blend 2 & 42 & $\mathrm{TL}$ & & & & & & TL Neph \\
\hline 1 & Case 15B - Blend 2 & 43 & TL Neph & & & & & Neph & TL Neph \\
\hline 1 & Case 15B - Blend 2 & 44 & TL Neph & TL & & TL & Neph & Neph & TL Neph \\
\hline 1 & Case 15B - Blend 2 & 45 & TL Neph & TL Neph & TL Neph & TL Neph & TL Neph & Neph & TL Neph \\
\hline 1 & Case 15B - Blend 2 & 46 & TL Neph & TL Neph & TL Neph & TL Neph & TL Neph & TL Neph & TL Neph \\
\hline 1 & Case $15 \mathrm{~B}$ - Blend 2 & 47 & TL Neph & TL Neph & TL Neph & TL Neph & TL Neph & TL Neph & TL Neph \\
\hline 1 & Case 15B - Blend 2 & 48 & TL Neph & TL Neph & TL Neph & TL Neph & TL Neph & TL Neph & TL Neph \\
\hline 1 & Case 15B - Blend 2 & 49 & TL newlv Neph & TL Neph & TL Neph & TL Neph & TL Neph & TL Neph & TL Neph \\
\hline 1 & Case $15 \mathrm{~B}$ - Blend 2 & 50 & TL newlv Neph & TL Neph & TL Neph & TL Neph & TL Neph & TL Neph & TL Neph \\
\hline 1 & Case 15B - Blend 2 & 51 & TL newlv Neph & TL Neph & TL Neph & TL Neph & TL Neph & TL Neph & TL Neph \\
\hline 1 & Case 15B - Blend 2 & 52 & TL newlv Neph & TL Neph & TL Neph & TL Neph & TL Neph & TL Neph & TL Neph \\
\hline 1 & Case 15B - Blend 2 & 53 & TL newlv Neph & TL Neph & TL Neph & TL Neph & TL Neph & TL Neph & TL Neph \\
\hline 1 & Case 15B - Blend 2 & 54 & TL newlv Neph & TL Neph & TL Neph & TL Neph & TL Neph & TL Neph & TL Neph \\
\hline 1 & Case 15B - Blend 2 & 55 & TL newlv Neph & TL Neph & TL Neph & TL Neph & TL Neph & TL Neph & TL Neph \\
\hline 1 & Case 15B - Blend 2 & 56 & TL newlv Neph & TL Neph & TL Neph & TL Neph & TL Neph & TL Neph & TL Neph \\
\hline 1 & Case 15B - Blend 2 & 57 & TL newlv Neph & TL Neph & TL Neph & TL Neph & TL Neph & TL Neph & TL Neph \\
\hline 1 & Case $15 \mathrm{~B}$ - Blend 2 & 58 & TL newlv Neph & TL newlv Neph & TL Neph & TL Neph & TL Neph & TL Neph & TL Neph \\
\hline 1 & Case 15B - Blend 2 & 59 & TL newlv Neph & TL newlv Neph & TL newlv Neph & TL Neph & TL Neph & TL Neph & TL Neph \\
\hline 1 & Case 15B - Blend 2 & 60 & TL newlv Neph & TL newlv Neph & TL newlv Neph & TL Neph & TL Neph & TL newlv Neph & TL Neph \\
\hline 1 & Case 16 - Batch 1 & 25 & Del Gp newlv Al2O3 R2O & Del Gp Al2O3 R2O & Del Gp Al2O3 R2O & Del Gp Al2O3 R2O & Del Gp Al2O3 R2O & Del Gp Al2O3 R2O & Del Gp Al2O3 R2O \\
\hline 1 & Case 16-Batch 1 & 26 & Del Gp newlv Al2O3 R2O & Del Gp newlv Al2O3 R2O & Del Gp Al2O3 R2O & Del Gp Al2O3 R2O & Del Gp Al2O3 R2O & Del Gp Al2O3 R2O & Del Gp Al2O3 R2O \\
\hline 1 & Case 16 - Batch 1 & 27 & Del Gp newlv Al2O3 R2O & Del Gp newlv Al2O3 R2O & Del Gp Al2O3 R2O & Del Gp Al2O3 R2O & Del Gp Al2O3 R2O & Del Gp Al2O3 R2O & Del Gp Al2O3 R2O \\
\hline 1 & Case 16 - Batch 1 & 28 & Del Gp newlv Al2O3 R2O & Del Gp newlv Al2O3 R2O & Del Gp newlv Al2O3 R2O & Del Gp Al2O3 R2O & Del Gp Al2O3 R2O & Del Gp Al2O3 R2O & Del Gp Al2O3 R2O \\
\hline 1 & Case 16 - Batch 1 & 29 & Del Gp newlv Al2O3 R2O & Del Gp newlv Al2O3 R2O & Del Gp newlv Al2O3 R2O & Del Gp Al2O3 R2O & Del Gp Al2O3 R2O & Del Gp newlv Al2O3 R2O & Del Gp Al2O3 R2O \\
\hline 1 & Case 16 - Batch 1 & 30 & Del Gp newlv Al2O3 R2O & Del Gp newlv Al2O3 R2O & Del Gp newlv Al2O3 R2O & Del Gp Al2O3 R2O & Del Gp Al2O3 R2O & Del Gp newlv Al2O3 R2O & Del Gp newlv Al2O3 R2O \\
\hline 1 & Case 16 - Batch 1 & 31 & Del Gp newlv Al2O3 R2O & Del Gp newlv Al2O3 R2O & Del Gp newlv Al2O3 R2O & Del Gp Al2O3 R2O & Del Gp newlv Al2O3 R2O & Del Gp newlv Al2O3 R2O & Del Gp newlv Al2O3 R2O \\
\hline 1 & Case 16 - Batch 1 & 32 & Del Gp newlv Al2O3 R2O & Del Gp newlv Al2O3 R2O & Del Gp newlv Al2O3 R2O & Del Gp Al2O3 R2O & Del Gp newlv Al2O3 R2O & Del Gp newlv Al2O3 R2O & Del Gp newlv Al2O3 R2O \\
\hline 1 & Case 16 - Batch 1 & 33 & Del Gp newlv Al2O3 R2O & Del Gp newlv Al2O3 R2O & Del Gp newlv Al2O3 R2O & Del Gp newlv Al2O3 R2O & Del Gp newlv Al2O3 R2O & Del Gp newlv Al2O3 R2O & Del Gp newlv Al2O3 R2O \\
\hline
\end{tabular}


Exhibit A1. Results for MAR Assessments of the Nominal Stage (part 3)

Constraint not met: neph (nepheline), TL (liquidus temperature), newlv (low viscosity - new model), newhv (high viscosity - new model), and Del GP - durability

\begin{tabular}{|c|c|c|c|c|c|c|c|c|c|}
\hline & & $\begin{array}{ll}\mathrm{Na} 2 \mathrm{O} \\
\end{array}$ & 0.07 & 0.07 & 0.07 & 0.08 & 0.09 & 0.1 & 0.11 \\
\hline Set & Type & Sludge Loading (\%) & P2-5 & P3-3 & P3-4 & 418 & 426 & 425 & 200 \\
\hline 1 & Case 16- Batch 1 & 34 & Del Gp newlv Al2O3 R2O & Del Gp newlv Al2O3 R2O & Del Gp newlv Al2O3 R2O & Del Gp newlv Al2O3 R2O & Del Gp newlv Al2O3 R2O & Del Gp newlv Al2O3 R2O & Del Gp newlv Al2O3 R2O \\
\hline$\frac{1}{1}$ & Case 16 - Batch 1 & 35 & Del Gp newlv Al2O3 R2O & Del Gp newlv Al2O3 R2O & Del Gp newlv Al2O3 R2O & Del Gp newlv Al2O3 R2O & Del Gp newlv Al2O3 R2O & Del Gp newlv Al2O3 R2O & Del Gp newlv Al2O3 R2O \\
\hline 1 & Case 16 - Batch 1 & 36 & Del Gp newlv Al2O3 R2O & Del Gp newlv Al2O3 R2O & Del Gp newlv Al2O3 R2O & Del Gp newlv Al2O3 R2O & Del Gp newlv Al2O3 R2O & Del Gp newlv Al2O3 R2O & Del Gp newlv Al2O3 R2O \\
\hline 1 & Case 16 - Batch 1 & 37 & Del Gp newlv Al2O3 R2O & Del Gp newlv Al2O3 R2O & Del Gp newlv Al2O3 R2O & Del Gp newlv Al2O3 R2O & Del Gp newlv Al2O3 R2O & Del Gp newlv Al2O3 R2O & Del Gp newlv Al2O3 R2O \\
\hline$\frac{1}{1}$ & Case 16 - Batch 1 & 38 & Del Gp newlv Al2O3 R2O & Del Gp newlv Al2O3 R2O & Del Gp newlv Al2O3 R2O & Del Gp newlv Al2O3 R2O & Del Gp newlv Al2O3 R2O & Del Gp newlv Al2O3 R2O & Del Gp newlv Al2O3 R2O \\
\hline 1 & Case 16 - Batch 1 & 39 & Del Gp newlv Al2O3 R2O & Del Gp newlv Al2O3 R2O & Del Gp newlv Al2O3 R2O & Del Gp newlv Al2O3 R2O & Del Gp newlv Al2O3 R2O & Del Gp newlv Al2O3 R2O & Del Gp newlv Al2O3 R2O \\
\hline 1 & Case 16 - Batch 1 & 40 & Del Gp newlv Al2O3 R2O & Del Gp newlv Al2O3 R2O & Del Gp newlv Al2O3 R2O & Del Gp newlv Al2O3 R2O & Del Gp newlv Al2O3 R2O & Del Gp newlv Al2O3 R2O & Del Gp newlv Al2O3 R2O \\
\hline 1 & Case 16 - Batch 1 & 41 & Del Gp newlv Al2O3 R2O & Del Gp newlv Al2O3 R2O & Del Gp newlv Al2O3 R2O & Del Gp newlv Al2O3 R2O & Del Gp newlv Al2O3 R2O & Del Gp newlv Al2O3 R2O & Del Gp newlv Al2O3 R2O \\
\hline$\frac{1}{1}$ & Case 16 - Batch 1 & 42 & Del Gp newlv Al2O3 R2O & Del Gp newlv Al2O3 R2O & Del Gp newlv Al2O3 R2O & Del Gp newlv Al2O3 R2O & Del Gp newlv Al2O3 R2O & Del Gp newlv Al2O3 R2O & Del Gp newlv Al2O3 R2O \\
\hline 1 & Case 16-Batch 1 & 43 & Del Gp newlv Al2O3 R2O & Del Gp newlv Al2O3 R2O & Del Gp newlv Al2O3 R2O & Del Gp newlv Al2O3 R2O & Del Gp newlv Al2O3 R2O & Del Gp newlv Al2O3 R2O & Del Gp newlv Al2O3 R2O \\
\hline 1 & Case 16 - Batch 1 & 44 & Del Gp newlv Al2O3 R2O & Del Gp newlv Al2O3 R2O & Del Gp newlv Al2O3 R2O & Del Gp newlv Al2O3 R2O & Del Gp newlv Al2O3 R2O & Del Gp newlv Al2O3 R2O & Del Gp newlv Al2O3 R2O \\
\hline 1 & Case 16 - Batch 1 & 45 & Del Gp newlv Al2O3 R2O & Del Gp newlv Al2O3 R2O & Del Gp newlv Al2O3 R2O & Del Gp newlv Al2O3 R2O & Del Gp newlv Al2O3 R2O & Del Gp newlv Al2O3 R2O & Del Gp newlv Al2O3 R2O \\
\hline 1 & Case 16 - Batch 1 & 46 & Del Gp newlv Al2O3 R2O & Del Gp newlv Al2O3 R2O & Del Gp newlv Al2O3 R2O & Del Gp newlv Al2O3 R2O & Del Gp newlv Al2O3 R2O & Del Gp newlv Al2O3 R2O & Del Gp newlv Al2O3 R2O \\
\hline 1 & Case 16 - Batch 1 & 47 & Del Gp newlv Al2O3 R2O & Del Gp newlv Al2O3 R2O & Del Gp newlv Al2O3 R2O & Del Gp newlv Al2O3 R2O & Del Gp newlv Al2O3 R2O & Del Gp newlv Al2O3 R2O & Del Gp newlv Al2O3 R2O Neph \\
\hline$\frac{1}{1}$ & Case 16 - Batch 1 & 48 & Del Gp newlv Al2O3 R2O & Del Gp newlv Al2O3 R2O & Del Gp newlv Al2O3 R2O & Del Gp newlv Al2O3 R2O & Del Gp newlv Al2O3 R2O & Del Gp newlv Al2O3 R2O & Del Gp newlv Al2O3 R2O Neph \\
\hline 1 & Case 16 - Batch 1 & 49 & Del Gp newlv Al2O3 R2O & Del Gp newlv Al2O3 R2O & Del Gp newlv Al2O3 R2O & Del Gp newlv Al2O3 R2O & Del Gp newlv Al2O3 R2O & Del Gp newlv Al2O3 R2O & Del Gp newlv Al2O3 R2O Neph \\
\hline 1 & Case 16 - Batch 1 & 50 & Del Gp newlv Al2O3 R2O Neph & Del Gp newlv Al2O3 R2O & Del Gp newlv Al2O3 R2O & Del Gp newlv Al2O3 R2O & Del Gp newlv Al2O3 R2O & Del Gp newlv Al2O3 R2O Neph & Del Gp newlv Al2O3 R2O Neph \\
\hline 1 & Case 16 - Batch 1 & 51 & Del Gp newlv R2O Neph & Del Gp newlv R2O & Del Gp newlv R2O & Del Gp newlv R2O & Del Gp newlv R2O Neph & Del Gp newlv R2O Neph & Del Gp newlv R2O Neph \\
\hline 1 & Case 16 - Batch 1 & 52 & Del Gp newlv Neph & Del Gp newlv R2O Neph & Del Gp newlv R2O Neph & Del Gp newlv R2O Neph & Del Gp newlv R2O Neph & Del Gp newlv R2O Neph & Del Gp newlv R2O Neph \\
\hline 1 & Case 16 - Batch 1 & 53 & Del Gp newlv Neph & Del Gp newlv R2O Neph & Del Gp newlv R2O Neph & Del Gp newlv R2O Neph & Del Gp newlv R2O Neph & Del Gp newlv R2O Neph & Del Gp newlv R2O Neph \\
\hline 1 & Case 16-Batch 1 & 54 & Del Gp newlv Neph & Del Gp newlv R2O Neph & Del Gp newlv R2O Neph & Del Gp newlv R2O Neph & Del Gp newlv R2O Neph & Del Gp newlv R2O Neph & Del Gp newlv R2O Neph \\
\hline 1 & Case 16 - Batch 1 & 55 & Del Gp newlv Neph & Del Gp newlv R2O Neph & Del Gp newlv R2O Neph & Del Gp newlv R2O Neph & Del Gp newlv R2O Neph & Del Gp newlv R2O Neph & Del Gp newlv R2O Neph \\
\hline 1 & Case 16 - Batch 1 & 56 & Del Gp newlv Neph & Del Gp newlv R2O Neph & Del Gp newlv R2O Neph & Del Gp newlv R2O Neph & Del Gp newlv R2O Neph & Del Gp newlv R2O Neph & Del Gp newlv R2O Neph \\
\hline 1 & Case 16 - Batch 1 & 57 & Del Gp newlv Neph & Del Gp newlv R2O Neph & Del Gp newlv R2O Neph & Del Gp newlv R2O Neph & Del Gp newlv R2O Neph & Del Gp newlv R2O Neph & Del Gp TL newlv R2O Neph \\
\hline 1 & Case 16 - Batch 1 & 58 & Del Gp newlv Neph & Del Gp newlv R2O Neph & Del Gp newlv R2O Neph & Del Gp newlv R2O Neph & Del Gp newlv R2O Neph & Del Gp newlv R2O Neph & Del Gp TL newlv R2O Neph \\
\hline$\frac{1}{1}$ & Case 16 - Batch 1 & 59 & Del Gp TL newlv Neph & Del Gp newlv R2O Neph & Del Gp newlv R2O Neph & Del Gp newlv R2O Neph & Del Gp newlv R2O Neph & Del Gp newlv R2O Neph & Del Gp TL newlv R2O Neph \\
\hline 1 & Case 16 - Batch 1 & 60 & Del Gp TL newlv Neph & Del Gp newlv R2O Neph & Del Gp newlv R2O Neph & Del Gp newlv R2O Neph & Del Gp newlv R2O Neph & Del Gp newlv R2O Neph & Del Gp TL newlv R2O Neph \\
\hline 1 & Case $16-$ Batch 2 & 25 & & & & newhy & newhy & & newhv \\
\hline 1 & Case 16 - Batch 2 & 26 & & & & newhv & newhy & & newhv \\
\hline 1 & Case 16 - Batch 2 & 27 & & & & newhv & newhv & & newhv \\
\hline 1 & Case 16 - Batch 2 & 28 & & & & newhv & & & newhv \\
\hline 1 & Case 16 - Batch 2 & 29 & & & & newhv & & & newhv \\
\hline 1 & Case 16 - Batch 2 & 30 & & & & newhv & & & newhv \\
\hline 1 & Case 16 - Batch 2 & 31 & & & & newhy & & & newhv \\
\hline 1 & Case 16 - Batch 2 & 32 & & & & newhv & & & \\
\hline 1 & Case 16 - Batch 2 & 33 & & & & newhv & & & \\
\hline 1 & Case 16 - Batch 2 & 34 & & & & newhy & & & \\
\hline$\frac{1}{1}$ & Case 16 - Batch 2 & 35 & & & & newhv & & & \\
\hline 1 & Case 16 - Batch 2 & 36 & & & & newhv & & & Neph \\
\hline$\frac{1}{1}$ & Case 16 - Batch 2 & 37 & & & & newhy & & & Neph \\
\hline$\frac{1}{1}$ & Case 16 - Batch 2 & 38 & & & & newhy & & & Neph \\
\hline 1 & Case 16 - Batch 2 & 39 & Neph & & & newhv & & Neph & Neph \\
\hline$\frac{1}{1}$ & Case 16 - Batch 2 & 40 & Neph & & & & Neph & Neph & Neph \\
\hline 1 & Case 16 - Batch 2 & 41 & Neph & Neph & Neph & Neph & Neph & Neph & Neph \\
\hline 1 & Case 16 - Batch 2 & 42 & Neph & Neph & Neph & Neph & Neph & Neph & Neph \\
\hline 1 & Case 16 - Batch 2 & 43 & Neph & Neph & Neph & Neph & Neph & Neph & Neph \\
\hline 1 & Case 16 - Batch 2 & 44 & Neph & Neph & Neph & Neph & Neph & Neph & Neph \\
\hline$\frac{1}{1}$ & Case 16 - Batch 2 & 45 & Neph & Neph & Neph & Neph & Neph & Neph & Neph \\
\hline 1 & Case 16 - Batch 2 & 46 & Neph & Neph & Neph & Neph & Neph & Neph & Neph \\
\hline 1 & Case 16 - Batch 2 & 47 & Neph & Neph & Neph & Neph & Neph & Neph & Neph \\
\hline 1 & Case 16 - Batch 2 & 48 & TL Neph & Neph & Neph & Neph & Neph & Neph & Neph \\
\hline
\end{tabular}


Exhibit A1. Results for MAR Assessments of the Nominal Stage (part 3)

Constraint not met: neph (nepheline), TL (liquidus temperature), newlv (low viscosity - new model), newhv (high viscosity - new model), and Del GP - durability

\begin{tabular}{|c|c|c|c|c|c|c|c|c|c|}
\hline & & $\mathrm{Na} 2 \mathrm{O}$ & 0.07 & 0.07 & 0.07 & 0.08 & 0.09 & 0.1 & 0.11 \\
\hline Set & Type & Sludge Loading (\%) & P2-5 & $\begin{array}{ll}\text { P3-3 } \\
\end{array}$ & P3-4 & 418 & 426 & 425 & 200 \\
\hline 1 & Case 16 - Batch 2 & 49 & TL Neph & Neph & Neph & Neph & Neph & Neph & TL Neph \\
\hline 1 & Case 16 - Batch 2 & 50 & TL Neph & Neph & Neph & Neph & Neph & Neph & TL Neph \\
\hline 1 & Case 16 - Batch 2 & 51 & TL Neph & Neph & Neph & Neph & Neph & Neph & TL Neph \\
\hline 1 & Case 16 - Batch 2 & 52 & TL Neph & Neph & Neph & Neph & Neph & Neph & TL Neph \\
\hline 1 & Case 16 - Batch 2 & 53 & TL Neph & TL Neph & Neph & TL Neph & Neph & Neph & TL Neph \\
\hline 1 & Case 16 - Batch 2 & 54 & TL Neph & TL Neph & TL Neph & TL Neph & TL Neph & Neph & TL Neph \\
\hline 1 & Case 16 - Batch 2 & 55 & TL Neph & TL Neph & TL Neph & TL Neph & TL Neph & TL Neph & TL Neph \\
\hline 1 & Case 16 - Batch 2 & 56 & TL Neph & TL Neph & TL Neph & TL Neph & TL Neph & TL Neph & TL Neph \\
\hline 1 & Case 16 - Batch 2 & 57 & TL Neph & TL Neph & TL Neph & TL Neph & TL Neph & TL Neph & TL Neph \\
\hline$\frac{1}{1}$ & Case 16 - Batch 2 & 58 & TL Neph & TL Neph & TL Neph & TL Neph & TL Neph & TL Neph & TL Neph \\
\hline 1 & Case 16 - Batch 2 & 59 & TL Neph & TL Neph & TL Neph & TL Neph & TL Neph & TL Neph & TL Neph \\
\hline 1 & Case 16 - Batch 2 & 60 & TL Neph & TL Neph & TL Neph & TL Neph & TL Neph & TL Neph & TL Neph \\
\hline 1 & Case 16 - Blend 1 & 25 & & & & & & & \\
\hline 1 & Case 16 - Blend 1 & 26 & & & & & & & \\
\hline 1 & Case 16 - Blend 1 & 27 & & & & & & & \\
\hline 1 & Case 16 - Blend 1 & 28 & & & & & & & \\
\hline 1 & Case 16 - Blend 1 & 29 & newlv & & & & & & \\
\hline 1 & Case 16 - Blend 1 & 30 & newlv & & & & & & \\
\hline 1 & Case 16 - Blend 1 & 31 & newlv & & & & & & \\
\hline 1 & Case 16 - Blend 1 & 32 & newlv & & & & & & \\
\hline$\frac{1}{1}$ & Case 16 - Blend 1 & 33 & newlv & & & & & & \\
\hline 1 & Case 16 - Blend 1 & 34 & newlv & & & & & & \\
\hline 1 & Case 16 - Blend 1 & 35 & newlv & & & & & & \\
\hline 1 & Case 16 - Blend 1 & 36 & newlv & & & & & & \\
\hline 1 & Case 16 - Blend 1 & 37 & newlv & newlv & & & & & \\
\hline 1 & Case 16 - Blend 1 & 38 & newlv & newlv & & & & & \\
\hline 1 & Case 16 - Blend 1 & 39 & TL newlv & newlv & newlv & & & & $\mathrm{TL}$ \\
\hline 1 & Case 16 - Blend 1 & 40 & TL newlv & newlv & newlv & & & newlv & $\mathrm{TL}$ \\
\hline 1 & Case 16 - Blend 1 & 41 & TL newlv & newlv & newlv & & & newlv & TL newlv \\
\hline 1 & Case 16 - Blend 1 & 42 & TL newlv & TL newlv & newlv & $\mathrm{TL}$ & newlv & newly & TL newly \\
\hline 1 & Case 16 - Blend 1 & 43 & TL newlv & TL newlv & TL newlv & $\mathrm{TL}$ & TL newlv & newlv & TL newlv \\
\hline 1 & Case 16 - Blend 1 & 44 & TL newlv & TL newlv & TL newlv & $\mathrm{TL}$ & TL newlv & TL newlv & TL newlv \\
\hline 1 & Case 16 - Blend 1 & 45 & TL newlv & TL newlv & TL newlv & TL newlv & TL newlv & TL newlv & TL newly \\
\hline 1 & Case 16 - Blend 1 & 46 & TL newlv & TL newlv & TL newlv & TL newlv & TL newlv & TL newlv & TL newlv \\
\hline 1 & Case 16 - Blend 1 & 47 & TL newlv & TL newlv & TL newlv & TL newlv & TL newlv & TL newlv & TL newlv \\
\hline 1 & Case 16 - Blend 1 & 48 & TL newlv & TL newlv & TL newlv & TL newlv & TL newlv & TL newlv & TL newlv \\
\hline 1 & Case 16 - Blend 1 & 49 & TL newlv & TL newlv & TL newlv & TL newlv & TL newlv & TL newlv & TL newlv \\
\hline 1 & Case 16 - Blend 1 & 50 & TL newlv & TL newlv & TL newlv & TL newlv & TL newlv & TL newlv & TL newlv \\
\hline 1 & Case 16 - Blend 1 & 51 & TL newlv & TL newlv & TL newlv & TL newlv & TL newlv & TL newlv & TL newlv Neph \\
\hline 1 & Case 16 - Blend 1 & 52 & TL newlv & TL newlv & TL newlv & TL newlv & TL newlv & TL newlv & TL newlv Neph \\
\hline 1 & Case 16 - Blend 1 & 53 & TL newlv & TL newlv & TL newlv & TL newlv & TL newlv & TL newlv Neph & TL newlv Neph \\
\hline 1 & Case 16 - Blend 1 & 54 & TL newlv Neph & TL newlv & TL newlv & TL newlv & TL newlv Neph & TL newlv Neph & TL newlv Neph \\
\hline 1 & Case 16 - Blend 1 & 55 & TL newlv Neph & TL newlv Neph & TL newlv & TL newlv & TL newlv Neph & TL newlv Neph & TL newlv Neph \\
\hline 1 & Case 16 - Blend 1 & 56 & TL newlv Neph & TL newlv Neph & TL newlv Neph & TL newlv Neph & TL newlv Neph & TL newlv Neph & TL newlv Neph \\
\hline 1 & Case 16 - Blend 1 & 57 & TL newlv Neph & TL newlv Neph & TL newlv Neph & TL newlv Neph & TL newlv Neph & TL newlv Neph & TL newlv Neph \\
\hline 1 & Case 16 - Blend 1 & 58 & TL newlv Neph & TL newlv Neph & TL newlv Neph & TL newlv Neph & TL newlv Neph & TL newlv Neph & TL newlv Neph \\
\hline 1 & Case 16 - Blend 1 & 59 & TL newlv Neph & TL newlv Neph & TL newlv Neph & TL newlv Neph & TL newlv Neph & TL newlv Neph & TL newlv Neph \\
\hline 1 & Case 16 - Blend 1 & 60 & TL newlv Neph & TL newlv Neph & TL newlv Neph & TL newlv Neph & TL newlv Neph & TL newlv Neph & TL newlv Neph \\
\hline 1 & Case 16 - Blend 2 & 25 & & & & newhv & & & \\
\hline 1 & Case 16 - Blend 2 & 26 & & & & & & & \\
\hline 1 & Case 16 - Blend 2 & 27 & & & & & & & \\
\hline
\end{tabular}


Exhibit A1. Results for MAR Assessments of the Nominal Stage (part 3)

Constraint not met: neph (nepheline), TL (liquidus temperature), newlv (low viscosity - new model), newhv (high viscosity - new model), and Del GP - durability

\begin{tabular}{|c|c|c|c|c|c|c|c|c|c|}
\hline & & $\mathrm{Na} 2 \mathrm{O}$ & 0.07 & 0.07 & 0.07 & 0.08 & 0.09 & 0.1 & 0.11 \\
\hline Set & Type & Sludge Loading (\%) & P2-5 & P3-3 & P3-4 & 418 & 426 & 425 & 200 \\
\hline 1 & Case 16 - Blend 2 & 28 & & & & & & & \\
\hline 1 & Case 16 - Blend 2 & 29 & & & & & & & \\
\hline 1 & Case 16 - Blend 2 & 30 & & & & & & & \\
\hline 1 & Case 16 - Blend 2 & 31 & & & & & & & \\
\hline 1 & Case 16 - Blend 2 & 32 & & & & & & & \\
\hline 1 & $\begin{array}{l}\text { Case } 16 \text { - Blend } 2 \\
\end{array}$ & 33 & & & & & & & \\
\hline 1 & Case 16 - Blend 2 & 34 & & & & & & & \\
\hline 1 & Case 16 - Blend 2 & 35 & & & & & & & \\
\hline 1 & Case 16 - Blend 2 & 36 & & & & & & & \\
\hline 1 & Case 16 - Blend 2 & 37 & & & & & & & \\
\hline 1 & Case 16 - Blend 2 & 38 & & & & & & & \\
\hline 1 & Case 16 - Blend 2 & 39 & & & & & & & Neph \\
\hline 1 & Case 16 - Blend 2 & 40 & & & & & & & Neph \\
\hline 1 & Case 16 - Blend 2 & 41 & & & & & & Neph & Neph \\
\hline 1 & Case 16 - Blend 2 & 42 & Neph & & & & Neph & Neph & Neph \\
\hline 1 & Case 16 - Blend 2 & 43 & Neph & Neph & & & Neph & Neph & Neph \\
\hline 1 & Case 16 - Blend 2 & 44 & TL Neph & Neph & Neph & Neph & Neph & Neph & Neph \\
\hline 1 & Case 16 - Blend 2 & 45 & TL Neph & Neph & Neph & Neph & Neph & Neph & TL Neph \\
\hline 1 & Case 16 - Blend 2 & 46 & TL Neph & Neph & Neph & Neph & Neph & Neph & TL Neph \\
\hline 1 & Case 16 - Blend 2 & 47 & TL Neph & Neph & Neph & Neph & Neph & Neph & TL Neph \\
\hline 1 & Case 16 - Blend 2 & 48 & TL Neph & TL Neph & Neph & Neph & Neph & Neph & TL Neph \\
\hline 1 & Case 16 - Blend 2 & 49 & TL newlv Neph & TL Neph & TL Neph & TL Neph & Neph & Neph & TL Neph \\
\hline 1 & Case 16 - Blend 2 & 50 & TL newlv Neph & TL Neph & TL Neph & TL Neph & TL Neph & TL Neph & TL Neph \\
\hline 1 & Case 16 - Blend 2 & 51 & TL newlv Neph & TL Neph & TL Neph & TL Neph & TL Neph & TL Neph & TL Neph \\
\hline 1 & Case 16 - Blend 2 & 52 & TL newlv Neph & TL Neph & TL Neph & TL Neph & TL Neph & TL Neph & TL Neph \\
\hline 1 & Case 16 - Blend 2 & 53 & TL newlv Neph & TL Neph & TL Neph & TL Neph & TL Neph & TL Neph & TL Neph \\
\hline 1 & Case 16 - Blend 2 & 54 & TL newlv Neph & TL Neph & TL Neph & TL Neph & TL Neph & TL Neph & TL Neph \\
\hline 1 & Case 16 - Blend 2 & 55 & TL newlv Neph & TL Neph & TL Neph & TL Neph & TL Neph & TL Neph & TL Neph \\
\hline 1 & Case 16 - Blend 2 & 56 & TL newlv Neph & TL Neph & TL Neph & TL Neph & TL Neph & TL Neph & TL Neph \\
\hline 1 & Case 16 - Blend 2 & 57 & TL newlv Neph & TL Neph & TL Neph & TL Neph & TL Neph & TL Neph & TL Neph \\
\hline 1 & Case 16 - Blend 2 & 58 & TL newlv Neph & TL newlv Neph & TL Neph & TL Neph & TL Neph & TL Neph & TL Neph \\
\hline 1 & Case 16 - Blend 2 & 59 & TL newlv Neph & TL newlv Neph & TL Neph & TL Neph & TL Neph & TL Neph & TL Neph \\
\hline 1 & Case 16 - Blend 2 & 60 & TL newlv Neph & TL newlv Neph & TL newlv Neph & TL Neph & TL Neph & TL Neph & TL Neph \\
\hline 1 & Case 7 Batch 1 & 25 & & & & & & & \\
\hline 1 & Case 7 Batch 1 & 26 & & & & & & & \\
\hline 1 & Case 7 Batch 1 & 27 & & & & & & & \\
\hline 1 & Case 7 Batch 1 & 28 & & & & & & & \\
\hline 1 & Case 7 Batch 1 & 29 & & & & & & & \\
\hline 1 & Case 7 Batch 1 & 30 & & & & & & & \\
\hline 1 & Case 7 Batch 1 & 31 & & & & & & & \\
\hline 1 & Case 7 Batch 1 & 32 & & & & & & & \\
\hline 1 & Case 7 Batch 1 & 33 & & & & & & & \\
\hline 1 & Case 7 Batch 1 & 34 & & & & & & & \\
\hline 1 & Case 7 Batch 1 & 35 & & & & & & & \\
\hline 1 & Case 7 Batch 1 & 36 & & & & & & & \\
\hline 1 & Case 7 Batch 1 & 37 & & & & & & & \\
\hline 1 & Case 7 Batch 1 & 38 & & & & & & & Neph \\
\hline 1 & Case 7 Batch 1 & 39 & & & & & & & Neph \\
\hline 1 & Case 7 Batch 1 & 40 & & & & & & Neph & Neph \\
\hline 1 & Case 7 Batch 1 & 41 & Neph & & & & Neph & Neph & Neph \\
\hline 1 & Case 7 Batch 1 & 42 & Neph & Neph & & Neph & Neph & Neph & Neph \\
\hline
\end{tabular}


Exhibit A1. Results for MAR Assessments of the Nominal Stage (part 3)

Constraint not met: neph (nepheline), TL (liquidus temperature), newlv (low viscosity - new model), newhv (high viscosity - new model), and Del GP - durability

\begin{tabular}{|c|c|c|c|c|c|c|c|c|c|}
\hline & & $\mathrm{Na} 2 \mathrm{O}$ & 0.07 & 0.07 & 0.07 & 0.08 & 0.09 & 0.1 & 0.11 \\
\hline Set & Type & Sludge Loading (\%) & $\mathrm{P} 2-5$ & $\begin{array}{ll}\text { P3-3 } \\
\end{array}$ & P3-4 & 418 & 426 & 425 & 200 \\
\hline 1 & Case 7 Batch 1 & 43 & Neph & Neph & Neph & Neph & Neph & Neph & Neph \\
\hline 1 & Case 7 Batch 1 & 44 & Neph & Neph & Neph & Neph & Neph & Neph & Neph \\
\hline 1 & Case 7 Batch 1 & 45 & Neph & Neph & Neph & Neph & Neph & Neph & Neph \\
\hline 1 & Case 7 Batch 1 & 46 & TL newlv Neph & Neph & Neph & Neph & Neph & Neph & Neph \\
\hline 1 & Case 7 Batch 1 & 47 & TL newlv Neph & Neph & Neph & Neph & Neph & Neph & TL Neph \\
\hline 1 & Case 7 Batch 1 & 48 & TL newlv Neph & Neph & Neph & Neph & Neph & Neph & TL Neph \\
\hline 1 & Case 7 Batch 1 & 49 & TL newlv Neph & Neph & Neph & Neph & Neph & Neph & TL Neph \\
\hline 1 & Case 7 Batch 1 & 50 & TL newlv Neph & Neph & Neph & Neph & Neph & Neph & TL Neph \\
\hline 1 & Case 7 Batch 1 & 51 & TL newlv Neph & TL Neph & Neph & TL Neph & Neph & Neph & TL Neph \\
\hline$\frac{1}{1}$ & Case 7 Batch 1 & 52 & TL newlv Neph & TL Neph & TL Neph & TL Neph & TL Neph & Neph & TL Neph \\
\hline 1 & Case 7 Batch 1 & 53 & TL newlv Neph & TL Neph & TL Neph & TL Neph & TL Neph & TL Neph & TL Neph \\
\hline 1 & Case 7 Batch 1 & 54 & TL newlv Neph & TL Neph & TL Neph & TL Neph & TL Neph & TL Neph & TL Neph \\
\hline 1 & Case 7 Batch 1 & 55 & TL newlv Neph & TL newlv Neph & TL Neph & TL Neph & TL Neph & TL Neph & TL Neph \\
\hline 1 & Case 7 Batch 1 & 56 & TL newlv Neph & TL newlv Neph & TL Neph & TL Neph & TL Neph & TL Neph & TL Neph \\
\hline 1 & Case 7 Batch 1 & 57 & TL newlv Neph & TL newlv Neph & TL newlv Neph & TL Neph & TL Neph & TL Neph & TL Neph \\
\hline 1 & Case 7 Batch 1 & 58 & TL newlv Neph & TL newlv Neph & TL newlv Neph & TL Neph & TL Neph & TL newlv Neph & TL Neph \\
\hline 1 & Case 7 Batch 1 & 59 & TL newlv Neph & TL newlv Neph & TL newlv Neph & TL Neph & TL Neph & TL newlv Neph & TL newlv Neph \\
\hline 1 & Case 7 Batch 1 & 60 & TL newlv Neph & TL newlv Neph & TL newlv Neph & TL Neph & TL newlv Neph & TL newlv Neph & TL newlv Neph \\
\hline 1 & Case 7 Batch 2 & 25 & & & & newhv & newhv & & newhv \\
\hline 1 & Case 7 Batch 2 & 26 & & & & newhv & & & newhy \\
\hline 1 & Case 7 Batch 2 & 27 & & & & newhy & & & newhy \\
\hline 1 & Case 7 Batch 2 & 28 & & & & newhv & & & newhv \\
\hline 1 & Case 7 Batch 2 & 29 & & & & newhy & & & newhv \\
\hline 1 & Case 7 Batch 2 & 30 & & & & newhv & & & \\
\hline 1 & Case 7 Batch 2 & 31 & & & & newhv & & & \\
\hline 1 & Case 7 Batch 2 & 32 & & & & newhy & & & \\
\hline 1 & Case 7 Batch 2 & 33 & & & & newhv & & & \\
\hline 1 & Case 7 Batch 2 & 34 & & & & newhy & & & \\
\hline 1 & Case 7 Batch 2 & 35 & & & & newhv & & & \\
\hline 1 & Case 7 Batch 2 & 36 & & & & newhv & & & \\
\hline 1 & Case 7 Batch 2 & 37 & & & & newhv & & & \\
\hline 1 & Case 7 Batch 2 & 38 & & & & & & & \\
\hline 1 & Case 7 Batch 2 & 39 & $\mathrm{TL}$ & & & & & & Neph \\
\hline 1 & Case 7 Batch 2 & 40 & $\mathrm{TL}$ & & & & & & TL Neph \\
\hline 1 & Case 7 Batch 2 & 41 & $\mathrm{TL}$ & & & & & Neph & TL Neph \\
\hline 1 & Case 7 Batch 2 & 42 & TL Neph & & & & & Neph & TL Neph \\
\hline 1 & Case 7 Batch 2 & 43 & TL Neph & TL Neph & & & Neph & Neph & TL Neph \\
\hline 1 & Case 7 Batch 2 & 44 & TL Neph & TL Neph & Neph & TL Neph & Neph & Neph & TL Neph \\
\hline 1 & Case 7 Batch 2 & 45 & TL Neph & TL Neph & TL Neph & TL Neph & TL Neph & Neph & TL Neph \\
\hline 1 & Case 7 Batch 2 & 46 & TL Neph & TL Neph & TL Neph & TL Neph & TL Neph & TL Neph & TL Neph \\
\hline 1 & Case 7 Batch 2 & 47 & TL Neph & TL Neph & TL Neph & TL Neph & TL Neph & TL Neph & TL Neph \\
\hline 1 & Case 7 Batch 2 & 48 & TL Neph & TL Neph & TL Neph & TL Neph & TL Neph & TL Neph & TL Neph \\
\hline 1 & Case 7 Batch 2 & 49 & TL Neph & TL Neph & TL Neph & TL Neph & TL Neph & TL Neph & TL Neph \\
\hline 1 & Case 7 Batch 2 & 50 & TL Neph & TL Neph & TL Neph & TL Neph & TL Neph & TL Neph & TL Neph \\
\hline 1 & Case 7 Batch 2 & 51 & TL Neph & TL Neph & TL Neph & TL Neph & TL Neph & TL Neph & TL Neph \\
\hline 1 & Case 7 Batch 2 & 52 & TL Neph & TL Neph & TL Neph & TL Neph & TL Neph & TL Neph & TL Neph \\
\hline 1 & Case 7 Batch 2 & 53 & TL Neph & TL Neph & TL Neph & TL Neph & TL Neph & TL Neph & TL Neph \\
\hline 1 & Case 7 Batch 2 & 54 & TL Neph & TL Neph & TL Neph & TL Neph & TL Neph & TL Neph & TL Neph \\
\hline 1 & Case 7 Batch 2 & 55 & TL Neph & TL Neph & TL Neph & TL Neph & TL Neph & TL Neph & TL Neph \\
\hline 1 & Case 7 Batch 2 & 56 & TL Neph & TL Neph & TL Neph & TL Neph & TL Neph & TL Neph & TL Neph \\
\hline 1 & Case 7 Batch 2 & 57 & TL Neph & TL Neph & TL Neph & TL Neph & TL Neph & TL Neph & TL Neph \\
\hline
\end{tabular}


Exhibit A1. Results for MAR Assessments of the Nominal Stage (part 3)

Constraint not met: neph (nepheline), TL (liquidus temperature), newlv (low viscosity - new model), newhv (high viscosity - new model), and Del GP - durability

\begin{tabular}{|c|c|c|c|c|c|c|c|c|c|}
\hline & & $\mathrm{Na} 2 \mathrm{O}$ & 0.07 & 0.07 & 0.07 & 0.08 & 0.09 & 0.1 & 0.11 \\
\hline Set & Type & Sludge Loading (\%) & P2-5 & P3-3 & P3-4 & 418 & 426 & 425 & 200 \\
\hline 1 & Case 7 Batch 2 & 58 & TL Neph & TL Neph & TL Neph & TL Neph & TL Neph & TL Neph & TL Neph \\
\hline 1 & Case 7 Batch 2 & 59 & TL Neph & TL Neph & TL Neph & TL Neph & TL Neph & TL Neph & TL Neph \\
\hline 1 & Case 7 Batch 2 & 60 & TL Neph & TL Neph & TL Neph & TL Neph & TL Neph & TL Neph & TL Neph \\
\hline 1 & Case 7 Blend 1 & 25 & & & & & & & \\
\hline 1 & Case 7 Blend 1 & 26 & & & & & & & \\
\hline 1 & Case 7 Blend 1 & 27 & & & & & & & \\
\hline 1 & Case 7 Blend 1 & 28 & & & & & & & \\
\hline 1 & Case 7 Blend 1 & 29 & & & & & & & \\
\hline 1 & Case 7 Blend 1 & 30 & newlv & & & & & & \\
\hline 1 & Case 7 Blend 1 & 31 & newlv & & & & & & \\
\hline 1 & Case 7 Blend 1 & 32 & newlv & & & & & & \\
\hline 1 & Case 7 Blend 1 & 33 & newlv & & & & & & \\
\hline 1 & Case 7 Blend 1 & 34 & newlv & & & & & & \\
\hline 1 & Case 7 Blend 1 & 35 & newlv & & & & & & \\
\hline 1 & Case 7 Blend 1 & 36 & newlv & & & & & & \\
\hline 1 & Case 7 Blend 1 & 37 & newlv & & & & & & \\
\hline 1 & Case 7 Blend 1 & 38 & newlv & newlv & & & & & \\
\hline 1 & Case 7 Blend 1 & 39 & newlv & newlv & & & & & \\
\hline 1 & Case 7 Blend 1 & 40 & newlv & newlv & newlv & & & & \\
\hline 1 & Case 7 Blend 1 & 41 & newlv & newlv & newlv & & & newlv & \\
\hline 1 & Case 7 Blend 1 & 42 & newlv & newlv & newlv & & & newlv & \\
\hline 1 & Case 7 Blend 1 & 43 & newlv & newlv & newlv & & & newlv & newlv \\
\hline 1 & Case 7 Blend 1 & 44 & TL newlv & newlv & newlv & & newlv & newlv & TL newly \\
\hline 1 & Case 7 Blend 1 & 45 & TL newlv & newlv & newlv & & newlv & newlv & TL newlv Neph \\
\hline 1 & Case 7 Blend 1 & 46 & TL newlv & newlv & newlv & newlv & newlv & newly & TL newlv Neph \\
\hline 1 & Case 7 Blend 1 & 47 & TL newlv & newlv & newlv & newlv & newlv & newlv & TL newlv Neph \\
\hline 1 & Case 7 Blend 1 & 48 & TL newlv Neph & TL newlv & newlv & TL newlv & newlv & newlv Neph & TL newlv Neph \\
\hline 1 & Case 7 Blend 1 & 49 & TL newlv Neph & TL newlv & TL newlv & TL newlv & TL newlv Neph & newlv Neph & TL newlv Neph \\
\hline 1 & Case 7 Blend 1 & 50 & TL newlv Neph & TL newlv Neph & TL newlv Neph & TL newlv Neph & TL newlv Neph & TL newlv Neph & TL newlv Neph \\
\hline 1 & Case 7 Blend 1 & 51 & TL newlv Neph & TL newlv Neph & TL newlv Neph & TL newlv Neph & TL newlv Neph & TL newlv Neph & TL newlv Neph \\
\hline 1 & Case 7 Blend 1 & 52 & TL newlv Neph & TL newlv Neph & TL newlv Neph & TL newlv Neph & TL newlv Neph & TL newlv Neph & TL newlv Neph \\
\hline 1 & Case 7 Blend 1 & 53 & TL newlv Neph & TL newlv Neph & TL newlv Neph & TL newlv Neph & TL newlv Neph & TL newlv Neph & TL newlv Neph \\
\hline 1 & Case 7 Blend 1 & 54 & TL newlv Neph & TL newlv Neph & TL newlv Neph & TL newlv Neph & TL newlv Neph & TL newlv Neph & TL newlv Neph \\
\hline 1 & Case 7 Blend 1 & 55 & TL newlv Neph & TL newlv Neph & TL newlv Neph & TL newlv Neph & TL newlv Neph & TL newlv Neph & TL newlv Neph \\
\hline 1 & Case 7 Blend 1 & 56 & TL newlv Neph & TL newlv Neph & TL newlv Neph & TL newlv Neph & TL newly Neph & TL newlv Neph & TL newlv Neph \\
\hline 1 & Case 7 Blend 1 & 57 & TL newlv Neph & TL newlv Neph & TL newlv Neph & TL newlv Neph & TL newlv Neph & TL newlv Neph & TL newlv Neph \\
\hline 1 & Case 7 Blend 1 & 58 & TL newlv Neph & TL newlv Neph & TL newlv Neph & TL newlv Neph & TL newlv Neph & TL newlv Neph & TL newlv Neph \\
\hline 1 & Case 7 Blend 1 & 59 & TL newlv Neph & TL newlv Neph & TL newlv Neph & TL newlv Neph & TL newlv Neph & TL newlv Neph & TL newlv Neph \\
\hline 1 & Case 7 Blend 1 & 60 & TL newlv Neph & TL newlv Neph & TL newlv Neph & TL newlv Neph & TL newlv Neph & TL newlv Neph & TL newlv Neph \\
\hline 1 & Case 7 Blend 2 & 25 & & & & newhv & & & \\
\hline 1 & Case 7 Blend 2 & 26 & & & & newhy & & & \\
\hline 1 & Case 7 Blend 2 & 27 & & & & & & & \\
\hline 1 & Case 7 Blend 2 & 28 & & & & & & & \\
\hline 1 & Case 7 Blend 2 & 29 & & & & & & & \\
\hline 1 & Case 7 Blend 2 & 30 & & & & & & & \\
\hline 1 & $\begin{array}{l}\text { Case } 7 \text { Blend } 2 \\
\end{array}$ & 31 & & & & & & & \\
\hline 1 & Case 7 Blend 2 & 32 & & & & & & & \\
\hline 1 & Case 7 Blend 2 & 33 & & & & & & & \\
\hline 1 & Case 7 Blend 2 & 34 & & & & & & & \\
\hline 1 & Case 7 Blend 2 & 35 & & & & & & & \\
\hline 1 & $\begin{array}{l}\text { Case } 7 \text { Blend } 2 \\
\end{array}$ & 36 & & & & & & & \\
\hline
\end{tabular}


Exhibit A1. Results for MAR Assessments of the Nominal Stage (part 3)

Constraint not met: neph (nepheline), TL (liquidus temperature), newlv (low viscosity - new model), newhv (high viscosity - new model), and Del GP - durability

\begin{tabular}{|c|c|c|c|c|c|c|c|c|c|}
\hline & & $\mathrm{Na} 2 \mathrm{O}$ & 0.07 & 0.07 & 0.07 & 0.08 & 0.09 & 0.1 & 0.11 \\
\hline Set & Type & $\begin{array}{l}\text { Sludge Loading (\%) } \\
\end{array}$ & $\mathrm{P2} 2-5$ & P3-3 & $\begin{array}{l}\text { P3-4 } \\
\end{array}$ & 418 & 426 & 425 & 200 \\
\hline 1 & Case 7 Blend 2 & 37 & & & & & & & \\
\hline 1 & Case 7 Blend 2 & 38 & & & & & & & \\
\hline 1 & Case 7 Blend 2 & 39 & & & & & & & \\
\hline 1 & Case 7 Blend 2 & 40 & $\mathrm{TL}$ & & & & & & Neph \\
\hline 1 & Case 7 Blend 2 & 41 & $\mathrm{TL}$ & & & & & & TL Neph \\
\hline 1 & Case 7 Blend 2 & 42 & $\mathrm{TL}$ & & & & & Neph & TL Neph \\
\hline 1 & Case 7 Blend 2 & 43 & TL Neph & & & & & Neph & TL Neph \\
\hline 1 & Case 7 Blend 2 & 44 & TL Neph & TL Neph & & & Neph & Neph & TL Neph \\
\hline 1 & Case 7 Blend 2 & 45 & TL Neph & TL Neph & TL Neph & TL Neph & Neph & Neph & TL Neph \\
\hline 1 & Case 7 Blend 2 & 46 & TL Neph & TL Neph & TL Neph & TL Neph & TL Neph & Neph & TL Neph \\
\hline 1 & Case 7 Blend 2 & 47 & TL Neph & TL Neph & TL Neph & TL Neph & TL Neph & TL Neph & TL Neph \\
\hline 1 & Case 7 Blend 2 & 48 & TL Neph & TL Neph & TL Neph & TL Neph & TL Neph & TL Neph & TL Neph \\
\hline 1 & Case 7 Blend 2 & 49 & TL Neph & TL Neph & TL Neph & TL Neph & TL Neph & TL Neph & TL Neph \\
\hline 1 & Case 7 Blend 2 & 50 & TL Neph & TL Neph & TL Neph & TL Neph & TL Neph & TL Neph & TL Neph \\
\hline 1 & Case 7 Blend 2 & 51 & TL newlv Neph & TL Neph & TL Neph & TL Neph & TL Neph & TL Neph & TL Neph \\
\hline 1 & Case 7 Blend 2 & 52 & TL newlv Neph & TL Neph & TL Neph & TL Neph & TL Neph & TL Neph & TL Neph \\
\hline 1 & Case 7 Blend 2 & 53 & TL newlv Neph & TL Neph & TL Neph & TL Neph & TL Neph & TL Neph & TL Neph \\
\hline 1 & Case 7 Blend 2 & 54 & TL newlv Neph & TL Neph & TL Neph & TL Neph & TL Neph & TL Neph & TL Neph \\
\hline 1 & Case 7 Blend 2 & 55 & TL newlv Neph & TL Neph & TL Neph & TL Neph & TL Neph & TL Neph & TL Neph \\
\hline 1 & Case 7 Blend 2 & 56 & TL newlv Neph & TL Neph & TL Neph & TL Neph & TL Neph & TL Neph & TL Neph \\
\hline 1 & Case 7 Blend 2 & 57 & TL newlv Neph & TL Neph & TL Neph & TL Neph & TL Neph & TL Neph & TL Neph \\
\hline 1 & Case 7 Blend 2 & 58 & TL newlv Neph & TL Neph & TL Neph & TL Neph & TL Neph & TL Neph & TL Neph \\
\hline 1 & Case 7 Blend 2 & 59 & TL newlv Neph & TL Neph & TL Neph & TL Neph & TL Neph & TL Neph & TL Neph \\
\hline 1 & Case 7 Blend 2 & 60 & TL newlv Neph & TL newlv Neph & TL Neph & TL Neph & TL Neph & TL Neph & TL Neph \\
\hline 1 & Case 8 - Blend 1 & 25 & & & & & & & \\
\hline 1 & Case 8 - Blend 1 & 26 & & & & & & & \\
\hline 1 & Case 8 - Blend 1 & 27 & & & & & & & \\
\hline 1 & Case 8 - Blend 1 & 28 & & & & & & & \\
\hline 1 & Case 8 - Blend 1 & 29 & & & & & & & \\
\hline 1 & Case 8 - Blend 1 & 30 & & & & & & & \\
\hline 1 & Case 8 - Blend 1 & 31 & & & & & & & \\
\hline 1 & Case 8 - Blend 1 & 32 & & & & & & & \\
\hline 1 & Case 8 - Blend 1 & 33 & & & & & & & \\
\hline 1 & Case 8 - Blend 1 & 34 & newlv & & & & & & \\
\hline 1 & Case 8 - Blend 1 & 35 & newlv & & & & & & \\
\hline 1 & Case 8 - Blend 1 & 36 & newlv & & & & & & \\
\hline 1 & Case 8 - Blend 1 & 37 & newlv & & & & & & \\
\hline 1 & Case 8 - Blend 1 & 38 & newlv & & & & & & \\
\hline 1 & Case 8 - Blend 1 & 39 & newlv & & & & & & \\
\hline 1 & Case 8 - Blend 1 & 40 & newlv & & & & & & \\
\hline 1 & Case 8 - Blend 1 & 41 & newlv & & & & & & \\
\hline 1 & Case 8 - Blend 1 & 42 & newlv & & & & & & \\
\hline 1 & Case 8 - Blend 1 & 43 & TL newlv & newlv & & & & & TL Neph \\
\hline 1 & Case 8 - Blend 1 & 44 & TL newlv & newlv & & & & & TL Neph \\
\hline 1 & Case 8 - Blend 1 & 45 & TL newlv Neph & $\begin{array}{l}\text { newlv } \\
\end{array}$ & newlv & & & Neph & TL Neph \\
\hline 1 & Case 8 - Blend 1 & 46 & TL newlv Neph & newlv & newlv & & Neph & newlv Neph & TL Neph \\
\hline 1 & Case 8 - Blend 1 & 47 & TL newlv Neph & TL newlv Neph & newlv Neph & TL Neph & Neph & newlv Neph & TL newlv Neph \\
\hline 1 & Case 8 - Blend 1 & 48 & TL newlv Neph & TL newlv Neph & TL newlv Neph & TL Neph & TL newlv Neph & newlv Neph & TL newlv Neph \\
\hline 1 & Case 8 - Blend 1 & 49 & TL newlv Neph & TL newlv Neph & TL newlv Neph & TL Neph & TL newlv Neph & TL newlv Neph & TL newlv Neph \\
\hline 1 & Case 8 - Blend 1 & 50 & TL newlv Neph & TL newlv Neph & TL newlv Neph & TL Neph & TL newlv Neph & TL newlv Neph & TL newlv Neph \\
\hline 1 & Case 8 - Blend 1 & 51 & TL newlv Neph & TL newlv Neph & TL newlv Neph & TL newlv Neph & TL newlv Neph & TL newlv Neph & TL newlv Neph \\
\hline
\end{tabular}


Exhibit A1. Results for MAR Assessments of the Nominal Stage (part 3)

Constraint not met: neph (nepheline), TL (liquidus temperature), newlv (low viscosity - new model), newhv (high viscosity - new model), and Del GP - durability

\begin{tabular}{|c|c|c|c|c|c|c|c|c|c|}
\hline & & $\mathrm{Na} 2 \mathrm{O}$ & 0.07 & 0.07 & 0.07 & 0.08 & 0.09 & 0.1 & 0.11 \\
\hline Set & Type & Sludge Loading (\%) & P2-5 & P3-3 & P3-4 & 418 & 426 & 425 & 200 \\
\hline 1 & Case 8 - Blend 1 & 52 & TL newlv Neph & TL newlv Neph & TL newlv Neph & TL newlv Neph & TL newlv Neph & TL newlv Neph & TL newlv Neph \\
\hline 1 & Case 8 - Blend 1 & 53 & TL newlv Neph & TL newlv Neph & TL newlv Neph & TL newlv Neph & TL newlv Neph & TL newlv Neph & TL newlv Neph \\
\hline 1 & Case 8 - Blend 1 & 54 & TL newlv Neph & TL newlv Neph & TL newlv Neph & TL newlv Neph & TL newlv Neph & TL newlv Neph & TL newlv Neph \\
\hline 1 & Case 8 - Blend 1 & 55 & TL newlv Neph & TL newlv Neph & TL newlv Neph & TL newlv Neph & TL newlv Neph & TL newlv Neph & TL newlv Neph \\
\hline 1 & Case 8 - Blend 1 & 56 & TL newlv Neph & TL newlv Neph & TL newlv Neph & TL newlv Neph & TL newlv Neph & TL newlv Neph & TL newlv Neph \\
\hline 1 & Case 8 - Blend 1 & 57 & TL newlv Neph & TL newlv Neph & TL newlv Neph & TL newlv Neph & TL newlv Neph & TL newlv Neph & TL newlv Neph \\
\hline 1 & Case 8 - Blend 1 & 58 & TL newlv Neph & TL newlv Neph & TL newlv Neph & TL newlv Neph & TL newlv Neph & TL newlv Neph & TL newlv Neph \\
\hline 1 & Case 8 - Blend 1 & 59 & TL newlv Neph & TL newlv Neph & TL newlv Neph & TL newlv Neph & TL newlv Neph & TL newlv Neph & TL newlv Neph \\
\hline 1 & Case 8 - Blend 1 & 60 & TL newlv Neph & TL newlv Neph & TL newlv Neph & TL newlv Neph & TL newlv Neph & TL newlv Neph & TL newlv Neph \\
\hline 1 & Case 8 and $8 a-$ Batch 1 & 25 & & & & & & & \\
\hline 1 & Case 8 and $8 \mathrm{a}-$ - Batch 1 & 26 & & & & & & & \\
\hline 1 & Case 8 and $8 a-$ Batch 1 & 27 & & & & & & & \\
\hline 1 & Case 8 and $8 a-$ Batch 1 & 28 & & & & & & & \\
\hline 1 & Case 8 and $8 \mathrm{a}-$ Batch 1 & 29 & & & & & & & \\
\hline 1 & Case 8 and $8 \mathrm{a}-$ - Batch 1 & 30 & & & & & & & \\
\hline 1 & Case 8 and $8 a-$ - Batch 1 & 31 & & & & & & & \\
\hline 1 & Case 8 and $8 \mathrm{a}$ - Batch 1 & 32 & & & & & & & \\
\hline 1 & Case 8 and $8 \mathrm{a}-$ - Batch 1 & 33 & & & & & & & \\
\hline 1 & Case 8 and $8 a-$ Batch 1 & 34 & & & & & & & \\
\hline 1 & Case 8 and $8 a-$ Batch 1 & 35 & & & & & & & \\
\hline 1 & Case 8 and $8 a-$ Batch 1 & 36 & & & & & & & \\
\hline 1 & Case 8 and $8 \mathrm{a}-$ - Batch 1 & 37 & & & & & & & \\
\hline 1 & Case 8 and $8 a-$ - Batch 1 & 38 & & & & & & & \\
\hline 1 & Case 8 and $8 a-$ Batch 1 & 39 & & & & & & & \\
\hline 1 & Case 8 and $8 a-$ Batch 1 & 40 & & & & & & & Neph \\
\hline 1 & Case 8 and $8 \mathrm{a}-$ - Batch 1 & 41 & & & & & & & Neph \\
\hline 1 & Case 8 and $8 a-$ Batch 1 & 42 & TL Neph & & & & & Neph & TL Neph \\
\hline 1 & Case 8 and $8 a-$ Batch 1 & 43 & TL newlv Neph & & & & Neph & Neph & TL Neph \\
\hline 1 & Case 8 and $8 \mathrm{a}-$ - Batch 1 & 44 & TL newlv Neph & Neph & Neph & Neph & Neph & Neph & TL Neph \\
\hline 1 & Case 8 and $8 \mathrm{a}-$ - Batch 1 & 45 & TL newlv Neph & Neph & Neph & Neph & Neph & Neph & TL Neph \\
\hline 1 & Case 8 and $8 a-$ Batch 1 & 46 & TL newlv Neph & TL Neph & Neph & TL Neph & Neph & Neph & TL Neph \\
\hline 1 & Case 8 and $8 \mathrm{a}-$ Batch 1 & 47 & TL newlv Neph & TL Neph & TL Neph & TL Neph & TL Neph & Neph & TL Neph \\
\hline 1 & Case 8 and $8 \mathrm{a}-$ - Batch 1 & 48 & TL newlv Neph & TL Neph & TL Neph & TL Neph & TL Neph & TL Neph & TL Neph \\
\hline 1 & Case 8 and $8 a-$ Batch 1 & 49 & TL newlv Neph & TL Neph & TL Neph & TL Neph & TL Neph & TL Neph & TL Neph \\
\hline 1 & Case 8 and $8 a-$ Batch 1 & 50 & TL newlv Neph & TL Neph & TL Neph & TL Neph & TL Neph & TL Neph & TL Neph \\
\hline 1 & Case 8 and $8 \mathrm{a}-$ - Batch 1 & 51 & TL newlv Neph & TL Neph & TL Neph & TL Neph & TL Neph & TL Neph & TL Neph \\
\hline 1 & Case 8 and $8 \mathrm{a}-$ - Batch 1 & 52 & TL newlv Neph & TL newlv Neph & TL Neph & TL Neph & TL Neph & TL Neph & TL Neph \\
\hline 1 & Case 8 and $8 a-$ Batch 1 & 53 & TL newlv Neph & TL newlv Neph & TL Neph & TL Neph & TL Neph & TL Neph & TL Neph \\
\hline 1 & Case 8 and $8 \mathrm{a}$ - Batch 1 & 54 & TL newlv Neph & TL newlv Neph & TL newlv Neph & TL Neph & TL Neph & TL Neph & TL Neph \\
\hline 1 & Case 8 and $8 \mathrm{a}-$ - Batch 1 & 55 & TL newlv Neph & TL newlv Neph & TL newlv Neph & TL Neph & TL Neph & TL newlv Neph & TL Neph \\
\hline 1 & Case 8 and $8 a-$ - Batch 1 & 56 & TL newlv Neph & TL newlv Neph & TL newlv Neph & TL Neph & TL Neph & TL newlv Neph & TL newlv Neph \\
\hline 1 & Case 8 and $8 a-$ Batch 1 & 57 & TL newlv Neph & TL newlv Neph & TL newlv Neph & TL Neph & TL newlv Neph & TL newlv Neph & TL newlv Neph \\
\hline 1 & Case 8 and $8 \mathrm{a}-$ - Batch 1 & 58 & TL newlv Neph & TL newlv Neph & TL newlv Neph & TL Neph & TL newlv Neph & TL newlv Neph & TL newlv Neph \\
\hline 1 & Case 8 and $8 a-$ Batch 1 & 59 & TL newlv Neph & TL newlv Neph & TL newlv Neph & TL newlv Neph & TL newlv Neph & TL newlv Neph & TL newlv Neph \\
\hline 1 & Case 8 and $8 a-$ Batch 1 & 60 & TL newlv Neph & TL newlv Neph & TL newlv Neph & TL newlv Neph & TL newlv Neph & TL newlv Neph & TL newlv Neph \\
\hline 1 & Case $8 \mathrm{~A}$ - Blend 1 & 25 & & & & & & & \\
\hline 1 & Case $8 \mathrm{~A}$ - Blend 1 & 26 & & & & & & & \\
\hline 1 & Case $8 \mathrm{~A}$ - Blend 1 & 27 & & & & & & & \\
\hline 1 & Case 8A - Blend 1 & 28 & & & & & & & \\
\hline 1 & Case $8 \mathrm{~A}$ - Blend 1 & 29 & & & & & & & \\
\hline 1 & Case $8 \mathrm{~A}-$ Blend 1 & 30 & & & & & & & \\
\hline
\end{tabular}


Exhibit A1. Results for MAR Assessments of the Nominal Stage (part 3)

Constraint not met: neph (nepheline), TL (liquidus temperature), newlv (low viscosity - new model), newhv (high viscosity - new model), and Del GP - durability

\begin{tabular}{|c|c|c|c|c|c|c|c|c|c|}
\hline & & $\mathrm{Na} 2 \mathrm{O}$ & 0.07 & 0.07 & 0.07 & 0.08 & 0.09 & 0.1 & 0.11 \\
\hline Set & Type & Sludge Loading (\%) & P2-5 & P3-3 & P3-4 & 418 & 426 & 425 & 200 \\
\hline 1 & Case $8 \mathrm{~A}$ - Blend 1 & 31 & & & & & & & \\
\hline 1 & Case $8 \mathrm{~A}$ - Blend 1 & 32 & & & & & & & \\
\hline 1 & Case $8 \mathrm{~A}$ - Blend 1 & 33 & & & & & & & \\
\hline 1 & Case $8 \mathrm{~A}$ - Blend 1 & 34 & & & & & & & \\
\hline 1 & Case $8 \mathrm{~A}$ - Blend 1 & 35 & & & & & & & \\
\hline 1 & Case 8A - Blend 1 & 36 & & & & & & & \\
\hline 1 & Case $8 \mathrm{~A}$ - Blend 1 & 37 & newlv & & & & & & \\
\hline 1 & Case 8A - Blend 1 & 38 & newlv & & & & & & \\
\hline 1 & Case $8 \mathrm{~A}$ - Blend 1 & 39 & newlv & & & & & & \\
\hline 1 & Case $8 \mathrm{~A}$ - Blend 1 & 40 & newlv & & & & & & \\
\hline 1 & Case 8A - Blend 1 & 41 & newlv & & & & & & Neph \\
\hline 1 & Case 8A - Blend 1 & 42 & TL newlv & & & & & & Neph \\
\hline 1 & Case $8 \mathrm{~A}$ - Blend 1 & 43 & TL newlv & & & & & Neph & TL Neph \\
\hline 1 & Case $8 \mathrm{~A}$ - Blend 1 & 44 & TL newlv Neph & & & & & Neph & TL Neph \\
\hline 1 & Case 8A - Blend 1 & 45 & TL newlv Neph & Neph & & & Neph & Neph & TL Neph \\
\hline 1 & Case $8 \mathrm{~A}$ - Blend 1 & 46 & TL newlv Neph & newlv Neph & Neph & Neph & Neph & Neph & TL Neph \\
\hline 1 & Case $8 \mathrm{~A}$ - Blend 1 & 47 & TL newlv Neph & TL newlv Neph & TL Neph & TL Neph & Neph & Neph & TL Neph \\
\hline 1 & Case 8A - Blend 1 & 48 & TL newlv Neph & TL newlv Neph & TL newlv Neph & TL Neph & TL Neph & Neph & TL Neph \\
\hline 1 & Case 8A - Blend 1 & 49 & TL newlv Neph & TL newlv Neph & TL newlv Neph & TL Neph & TL Neph & TL newlv Neph & TL Neph \\
\hline$\frac{1}{1}$ & Case $8 \mathrm{~A}$ - Blend 1 & 50 & TL newlv Neph & TL newlv Neph & TL newlv Neph & TL Neph & TL Neph & TL newlv Neph & TL Neph \\
\hline 1 & Case $8 \mathrm{~A}$ - Blend 1 & 51 & TL newlv Neph & TL newlv Neph & TL newlv Neph & TL Neph & TL Neph & TL newlv Neph & TL newlv Neph \\
\hline 1 & Case 8A - Blend 1 & 52 & TL newlv Neph & TL newlv Neph & TL newlv Neph & TL Neph & TL newlv Neph & TL newlv Neph & TL newlv Neph \\
\hline 1 & Case $8 \mathrm{~A}$ - Blend 1 & 53 & TL newlv Neph & TL newlv Neph & TL newlv Neph & TL Neph & TL newlv Neph & TL newlv Neph & TL newlv Neph \\
\hline 1 & Case 8A - Blend 1 & 54 & TL newlv Neph & TL newlv Neph & TL newlv Neph & TL newlv Neph & TL newlv Neph & TL newlv Neph & TL newlv Neph \\
\hline 1 & Case $8 \mathrm{~A}$ - Blend 1 & 55 & TL newlv Neph & TL newlv Neph & TL newlv Neph & TL newlv Neph & TL newlv Neph & TL newlv Neph & TL newlv Neph \\
\hline 1 & Case $8 \mathrm{~A}$ - Blend 1 & 56 & TL newlv Neph & TL newlv Neph & TL newlv Neph & TL newlv Neph & TL newlv Neph & TL newlv Neph & TL newlv Neph \\
\hline 1 & Case $8 \mathrm{~A}$ - Blend 1 & 57 & TL newlv Neph & TL newlv Neph & TL newlv Neph & TL newlv Neph & TL newlv Neph & TL newlv Neph & TL newlv Neph \\
\hline 1 & Case 8A - Blend 1 & 58 & TL newlv Neph & TL newlv Neph & TL newlv Neph & TL newlv Neph & TL newlv Neph & TL newlv Neph & TL newlv Neph \\
\hline 1 & Case 8A - Blend 1 & 59 & TL newlv Neph & TL newlv Neph & TL newlv Neph & TL newlv Neph & TL newlv Neph & TL newlv Neph & TL newlv Neph \\
\hline 1 & Case $8 \mathrm{~A}$ - Blend 1 & 60 & TL newlv Neph & TL newlv Neph & TL newlv Neph & TL newlv Neph & TL newlv Neph & TL newlv Neph & TL newlv Neph \\
\hline 2 & Case 15 Batch 1 & 25 & & newhv & newhv & newhv & newhv & newhy & newhv \\
\hline 2 & Case 15 Batch 1 & 26 & & newhv & newhv & newhv & newhv & newhv & newhv \\
\hline 2 & Case 15 Batch 1 & 27 & & newhv & newhv & newhv & newhv & newhy & newhv \\
\hline 2 & Case 15 Batch 1 & 28 & & newhv & newhv & newhy & newhv & newhv & newhv \\
\hline 2 & Case 15 Batch 1 & 29 & & newhy & newhv & newhv & newhv & newhv & newhv \\
\hline 2 & Case 15 Batch 1 & 30 & & newhv & newhy & newhv & newhy & newhy & newhy \\
\hline 2 & Case 15 Batch 1 & 31 & & newhy & newhv & newhv & newhv & newhy & newhv \\
\hline 2 & Case 15 Batch 1 & 32 & & newhv & newhy & newhy & newhy & newhy & newhv \\
\hline 2 & Case 15 Batch 1 & 33 & & newhv & newhv & newhv & newhv & newhv & newhv \\
\hline 2 & Case 15 Batch 1 & 34 & & newhv & newhy & newhy & newhy & newhv & newhy \\
\hline 2 & $\begin{array}{l}\text { Case } 15 \text { Batch } 1 \\
\end{array}$ & 35 & & newhv & newhv & newhv & newhv & newhv & newhv \\
\hline 2 & Case 15 Batch 1 & 36 & & newhv & newhv & newhv & newhv & newhv & newhv Neph \\
\hline 2 & Case 15 Batch 1 & 37 & & newhv & newhy & newhy & newhy & newhy & newhv Neph \\
\hline 2 & Case 15 Batch 1 & 38 & & newhv & newhv & newhv & newhv & newhy Neph & newhv Neph \\
\hline 2 & Case 15 Batch 1 & 39 & Neph & newhv & newhy & newhv & newhv Neph & newhv Neph & newhv Neph \\
\hline 2 & Case 15 Batch 1 & 40 & Neph & newhv Neph & newhv & newhv Neph & newhv Neph & newhy Neph & newhv Neph \\
\hline 2 & Case 15 Batch 1 & 41 & Neph & newhv Neph & newhv Neph & newhv Neph & newhv Neph & newhv Neph & newhv Neph \\
\hline 2 & $\begin{array}{l}\text { Case } 15 \text { Batch } 1 \\
\end{array}$ & 42 & Neph & newhv Neph & newhv Neph & newhv Neph & newhv Neph & newhv Neph & newhv Neph \\
\hline 2 & Case 15 Batch 1 & 43 & Neph & newhv Neph & newhv Neph & newhv Neph & newhv Neph & newhv Neph & newhv Neph \\
\hline 2 & Case 15 Batch 1 & 44 & Neph & newhv Neph & newhv Neph & newhv Neph & newhv Neph & newhv Neph & newhv Neph \\
\hline 2 & Case 15 Batch 1 & 45 & Neph & newhv Neph & newhv Neph & newhy Neph & newhv Neph & newhv Neph & newhv Neph \\
\hline
\end{tabular}


Exhibit A1. Results for MAR Assessments of the Nominal Stage (part 3)

Constraint not met: neph (nepheline), TL (liquidus temperature), newlv (low viscosity - new model), newhv (high viscosity - new model), and Del GP - durability

\begin{tabular}{|c|c|c|c|c|c|c|c|c|c|}
\hline & & $\mathrm{Na} 2 \mathrm{O}$ & 0.07 & 0.07 & 0.07 & 0.08 & 0.09 & 0.1 & 0.11 \\
\hline Set & Type & $\begin{array}{l}\text { Sludge Loading (\%) } \\
\end{array}$ & $\begin{array}{l}\mathrm{P} 2-5 \\
\end{array}$ & P3-3 & P3-4 & 418 & 426 & 425 & 200 \\
\hline 2 & Case 15 Batch 1 & 46 & TL Neph & newhv Neph & newhv Neph & newhv Neph & newhv Neph & newhv Neph & newhv Neph \\
\hline 2 & Case 15 Batch 1 & 47 & TL Neph & newhv Neph & newhv Neph & newhv Neph & newhv Neph & newhv Neph & newhv Neph \\
\hline 2 & Case 15 Batch 1 & 48 & TL newhv Neph & newhv Neph & newhv Neph & newhv Neph & newhv Neph & newhv Neph & TL newhy Neph \\
\hline 2 & Case 15 Batch 1 & 49 & TL newhv Neph & newhv Neph & newhv Neph & newhv Neph & newhv Neph & newhv Neph & TL newhy Neph \\
\hline 2 & Case 15 Batch 1 & 50 & TL newhy Neph & newhv Neph & newhv Neph & newhv Neph & newhv Neph & newhv Neph & TL newhy Neph \\
\hline 2 & $\begin{array}{l}\text { Case } 15 \text { Batch } 1 \\
\end{array}$ & 51 & TL newhv Neph & TL newhv Neph & newhv Neph & newhv Neph & newhv Neph & newhv Neph & TL newhy Neph \\
\hline 2 & Case 15 Batch 1 & 52 & TL newhv Neph & TL newhv Neph & newhv Neph & TL newhv Neph & newhv Neph & newhv Neph & TL newhy Neph \\
\hline 2 & Case 15 Batch 1 & 53 & TL newhv Neph & TL newhv Neph & TL newhv Neph & TL newhv Neph & TL newhv Neph & newhv Neph & TL newhy Neph \\
\hline 2 & Case 15 Batch 1 & 54 & TL newhy Neph & TL newhy Neph & TL newhv Neph & TL newhv Neph & TL newhy Neph & TL newhy Neph & TL newhv Neph \\
\hline 2 & Case 15 Batch 1 & 55 & TL newhv Neph & TL newhv Neph & TL newhy Neph & TL newhv Neph & TL newhy Neph & TL newhv Neph & TL newhy Neph \\
\hline 2 & Case 15 Batch 1 & 56 & TL newhv Neph & TL newhv Neph & TL newhv Neph & TL newhv Neph & TL newhv Neph & TL newhv Neph & TL newhy Neph \\
\hline 2 & Case 15 Batch 1 & 57 & TL newhv Neph & TL newhy Neph & TL newhy Neph & TL newhy Neph & TL newhv Neph & TL newhv Neph & TL newhy Neph \\
\hline 2 & Case 15 Batch 1 & 58 & TL newhy Neph & TL newhy Neph & TL newhy Neph & TL newhy Neph & TL newhy Neph & TL newhy Neph & TL newhy Neph \\
\hline 2 & Case 15 Batch 1 & 59 & TL newhv Neph & TL newhv Neph & TL newhv Neph & TL newhv Neph & TL newhy Neph & TL newhv Neph & TL newhy Neph \\
\hline 2 & Case 15 Batch 1 & 60 & TL newhv Neph & TL newhv Neph & TL newhy Neph & TL newhv Neph & TL newhv Neph & TL newhy Neph & TL newhy Neph \\
\hline 2 & Case 15 Batch 2 & 25 & & & & & & & \\
\hline 2 & Case 15 Batch 2 & 26 & & & & & & & \\
\hline 2 & Case 15 Batch 2 & 27 & & & & & & & \\
\hline 2 & Case 15 Batch 2 & 28 & & & & & & & \\
\hline 2 & Case 15 Batch 2 & 29 & & & & & & & \\
\hline 2 & Case 15 Batch 2 & 30 & & & & & & & \\
\hline 2 & Case 15 Batch 2 & 31 & & & & & & & \\
\hline 2 & Case 15 Batch 2 & 32 & & & & & & & \\
\hline 2 & Case 15 Batch 2 & 33 & & & & & & & \\
\hline 2 & Case 15 Batch 2 & 34 & & & & & & & \\
\hline 2 & Case 15 Batch 2 & 35 & & & & & & & \\
\hline 2 & Case 15 Batch 2 & 36 & & & & & & & \\
\hline 2 & $\begin{array}{l}\text { Case } 15 \text { Batch } 2 \\
\end{array}$ & 37 & & & & & & & \\
\hline 2 & Case 15 Batch 2 & 38 & & & & & & & \\
\hline 2 & Case 15 Batch 2 & 39 & & & & & & & Neph \\
\hline 2 & Case 15 Batch 2 & 40 & newlv & & & & & & Neph \\
\hline 2 & Case 15 Batch 2 & 41 & newlv & & & & & & Neph \\
\hline 2 & Case 15 Batch 2 & 42 & newlv Neph & & & & & Neph & Neph \\
\hline 2 & Case 15 Batch 2 & 43 & TL newlv Neph & & & & Neph & Neph & Neph \\
\hline 2 & Case 15 Batch 2 & 44 & TL newlv Neph & Neph & Neph & Neph & Neph & Neph & TL Neph \\
\hline 2 & Case 15 Batch 2 & 45 & TL newlv Neph & Neph & Neph & Neph & Neph & Neph & TL Neph \\
\hline 2 & Case 15 Batch 2 & 46 & TL newlv Neph & Neph & Neph & Neph & Neph & Neph & TL Neph \\
\hline 2 & Case 15 Batch 2 & 47 & TL newlv Neph & TL Neph & Neph & Neph & Neph & Neph & TL Neph \\
\hline 2 & $\begin{array}{l}\text { Case } 15 \text { Batch } 2 \\
\end{array}$ & 48 & TL newlv Neph & TL Neph & TL Neph & TL Neph & Neph & Neph & TL Neph \\
\hline 2 & Case 15 Batch 2 & 49 & TL newlv Neph & TL newlv Neph & TL Neph & TL Neph & TL Neph & Neph & TL Neph \\
\hline 2 & Case 15 Batch 2 & 50 & TL newlv Neph & TL newlv Neph & TL Neph & TL Neph & TL Neph & TL Neph & TL Neph \\
\hline 2 & Case 15 Batch 2 & 51 & TL newlv Neph & TL newlv Neph & TL newlv Neph & TL Neph & TL Neph & TL Neph & TL Neph \\
\hline 2 & Case 15 Batch 2 & 52 & TL newlv Neph & TL newlv Neph & TL newlv Neph & TL Neph & TL Neph & TL newlv Neph & TL Neph \\
\hline 2 & Case 15 Batch 2 & 53 & TL newlv Neph & TL newlv Neph & TL newlv Neph & TL Neph & TL Neph & TL newlv Neph & TL Neph \\
\hline 2 & Case 15 Batch 2 & 54 & TL newlv Neph & TL newlv Neph & TL newlv Neph & TL Neph & TL Neph & TL newlv Neph & TL newlv Neph \\
\hline 2 & Case 15 Batch 2 & 55 & TL newlv Neph & TL newlv Neph & TL newlv Neph & TL Neph & TL newlv Neph & TL newlv Neph & TL newlv Neph \\
\hline 2 & Case 15 Batch 2 & 56 & TL newlv Neph & TL newlv Neph & TL newlv Neph & TL Neph & TL newlv Neph & TL newlv Neph & TL newlv Neph \\
\hline 2 & Case 15 Batch 2 & 57 & TL newlv Neph & TL newlv Neph & TL newlv Neph & TL newlv Neph & TL newlv Neph & TL newlv Neph & TL newlv Neph \\
\hline 2 & Case 15 Batch 2 & 58 & TL newlv Neph & TL newlv Neph & TL newlv Neph & TL newlv Neph & TL newlv Neph & TL newlv Neph & TL newlv Neph \\
\hline 2 & $\begin{array}{l}\text { Case } 15 \text { Batch } 2 \\
\end{array}$ & 59 & TL newlv Neph & TL newlv Neph & TL newlv Neph & TL newlv Neph & TL newlv Neph & TL newlv Neph & TL newlv Neph \\
\hline 2 & Case 15 Batch 2 & 60 & TL newlv Neph & TL newlv Neph & TL newlv Neph & TL newlv Neph & TL newlv Neph & TL newlv Neph & TL newlv Neph \\
\hline
\end{tabular}


Exhibit A1. Results for MAR Assessments of the Nominal Stage (part 3)

Constraint not met: neph (nepheline), TL (liquidus temperature), newlv (low viscosity - new model), newhv (high viscosity - new model), and Del GP - durability

\begin{tabular}{|c|c|c|c|c|c|c|c|c|c|}
\hline & & $\mathrm{Na} 2 \mathrm{O}$ & 0.07 & 0.07 & 0.07 & 0.08 & 0.09 & 0.1 & 0.11 \\
\hline Set & Type & $\begin{array}{l}\text { Sludge Loading (\%) } \\
\end{array}$ & $\mathrm{P2} 2-5$ & P3-3 & $\begin{array}{l}\text { P3-4 } \\
\end{array}$ & 418 & 426 & 425 & 200 \\
\hline 2 & Case 15 Blend 1 & 25 & & & & & & & \\
\hline 2 & Case 15 Blend 1 & 26 & & & & & & & \\
\hline 2 & Case 15 Blend 1 & 27 & & & & & & & \\
\hline 2 & Case 15 Blend 1 & 28 & & & & & & & \\
\hline 2 & Case 15 Blend 1 & 29 & & & & & & & \\
\hline 2 & Case 15 Blend 1 & 30 & & & & & & & \\
\hline 2 & Case 15 Blend 1 & 31 & & & & & & & \\
\hline 2 & Case 15 Blend 1 & 32 & & & & & & & \\
\hline 2 & Case 15 Blend 1 & 33 & & & & & & & \\
\hline 2 & Case 15 Blend 1 & 34 & & & & & & & \\
\hline 2 & Case 15 Blend 1 & 35 & & & & & & & \\
\hline 2 & Case 15 Blend 1 & 36 & & & & & & & \\
\hline 2 & Case 15 Blend 1 & 37 & & & & & & & \\
\hline 2 & Case 15 Blend 1 & 38 & & & & & & & \\
\hline 2 & Case 15 Blend 1 & 39 & & & & & & & \\
\hline 2 & Case 15 Blend 1 & 40 & newlv & & & & & & \\
\hline 2 & Case 15 Blend 1 & 41 & newlv & & & & & & \\
\hline 2 & Case 15 Blend 1 & 42 & TL newlv & & & & & & \\
\hline 2 & Case 15 Blend 1 & 43 & TL newlv & & & & & & TL Neph \\
\hline 2 & Case 15 Blend 1 & 44 & TL newlv & & & & & & TL Neph \\
\hline 2 & Case 15 Blend 1 & 45 & TL newlv & & & & & Neph & TL Neph \\
\hline 2 & Case 15 Blend 1 & 46 & TL newlv Neph & $\mathrm{TL}$ & & & & Neph & TL Neph \\
\hline 2 & Case 15 Blend 1 & 47 & TL newlv Neph & TL Neph & $\mathrm{TL}$ & $\mathrm{TL}$ & Neph & Neph & TL Neph \\
\hline 2 & Case 15 Blend 1 & 48 & TL newlv Neph & TL Neph & TL Neph & TL Neph & TL Neph & TL Neph & TL Neph \\
\hline 2 & Case 15 Blend 1 & 49 & TL newlv Neph & TL newlv Neph & TL Neph & TL Neph & TL Neph & TL Neph & TL Neph \\
\hline 2 & Case 15 Blend 1 & 50 & TL newlv Neph & TL newlv Neph & TL Neph & TL Neph & TL Neph & TL Neph & TL Neph \\
\hline 2 & Case 15 Blend 1 & 51 & TL newlv Neph & TL newlv Neph & TL newlv Neph & TL Neph & TL Neph & TL newly Neph & TL Neph \\
\hline 2 & Case 15 Blend 1 & 52 & TL newlv Neph & TL newlv Neph & TL newlv Neph & TL Neph & TL Neph & TL newlv Neph & TL Neph \\
\hline 2 & Case 15 Blend 1 & 53 & TL newlv Neph & TL newlv Neph & TL newlv Neph & TL Neph & TL Neph & TL newly Neph & TL newlv Neph \\
\hline 2 & Case 15 Blend 1 & 54 & TL newlv Neph & TL newlv Neph & TL newlv Neph & TL Neph & TL newlv Neph & TL newlv Neph & TL newlv Neph \\
\hline 2 & Case 15 Blend 1 & 55 & TL newly Neph & TL newlv Neph & TL newlv Neph & TL Neph & TL newlv Neph & TL newlv Neph & TL newlv Neph \\
\hline 2 & Case 15 Blend 1 & 56 & TL newlv Neph & TL newlv Neph & TL newlv Neph & TL newlv Neph & TL newlv Neph & TL newlv Neph & TL newlv Neph \\
\hline 2 & Case 15 Blend 1 & 57 & TL newlv Neph & TL newlv Neph & TL newlv Neph & TL newlv Neph & TL newlv Neph & TL newlv Neph & TL newlv Neph \\
\hline 2 & Case 15 Blend 1 & 58 & TL newlv Neph & TL newlv Neph & TL newlv Neph & TL newlv Neph & TL newlv Neph & TL newlv Neph & TL newlv Neph \\
\hline 2 & Case 15 Blend 1 & 59 & TL newlv Neph & TL newlv Neph & TL newlv Neph & TL newlv Neph & TL newlv Neph & TL newlv Neph & TL newlv Neph \\
\hline 2 & Case 15 Blend 1 & 60 & TL newly Neph & TL newlv Neph & TL newlv Neph & TL newlv Neph & TL newlv Neph & TL newlv Neph & TL newlv Neph \\
\hline 2 & Case 15 Blend 2 & 25 & & & & & & & \\
\hline 2 & Case 15 Blend 2 & 26 & & & & & & & \\
\hline 2 & Case 15 Blend 2 & 27 & & & & & & & \\
\hline 2 & Case 15 Blend 2 & 28 & & & & & & & \\
\hline 2 & Case 15 Blend 2 & 29 & & & & & & & \\
\hline 2 & Case 15 Blend 2 & 30 & & & & & & & \\
\hline 2 & Case 15 Blend 2 & 31 & & & & & & & \\
\hline 2 & Case 15 Blend 2 & 32 & & & & & & & \\
\hline 2 & Case 15 Blend 2 & 33 & & & & & & & \\
\hline 2 & Case 15 Blend 2 & 34 & & & & & & & \\
\hline 2 & Case 15 Blend 2 & 35 & & & & & & & \\
\hline 2 & Case 15 Blend 2 & 36 & & & & & & & \\
\hline 2 & Case 15 Blend 2 & 37 & & & & & & & \\
\hline 2 & Case 15 Blend 2 & 38 & & & & & & & \\
\hline 2 & Case 15 Blend 2 & 39 & newlv & & & & & & \\
\hline
\end{tabular}


Exhibit A1. Results for MAR Assessments of the Nominal Stage (part 3)

Constraint not met: neph (nepheline), TL (liquidus temperature), newlv (low viscosity - new model), newhv (high viscosity - new model), and Del GP - durability

\begin{tabular}{|c|c|c|c|c|c|c|c|c|c|}
\hline & & $\mathrm{Na} 2 \mathrm{O}$ & 0.07 & 0.07 & 0.07 & 0.08 & 0.09 & 0.1 & 0.11 \\
\hline Set & Type & Sludge Loading (\%) & $\begin{array}{ll}\mathrm{P} 2-5 \\
\end{array}$ & P3-3 & P3-4 & 418 & 426 & 425 & 200 \\
\hline 2 & Case 15 Blend 2 & 40 & newlv & & & & & & Neph \\
\hline 2 & Case 15 Blend 2 & 41 & newlv & & & & & & Neph \\
\hline 2 & Case 15 Blend 2 & 42 & newlv & & & & & Neph & Neph \\
\hline 2 & Case 15 Blend 2 & 43 & TL newlv Neph & & & & Neph & Neph & Neph \\
\hline 2 & Case 15 Blend 2 & 44 & TL newlv Neph & Neph & & Neph & Neph & Neph & TL Neph \\
\hline 2 & Case 15 Blend 2 & 45 & TL newlv Neph & Neph & Neph & Neph & Neph & Neph & TL Neph \\
\hline 2 & Case 15 Blend 2 & 46 & TL newlv Neph & Neph & Neph & Neph & Neph & Neph & TL Neph \\
\hline 2 & Case 15 Blend 2 & 47 & TL newlv Neph & Neph & Neph & Neph & Neph & Neph & TL Neph \\
\hline 2 & Case 15 Blend 2 & 48 & TL newlv Neph & TL newlv Neph & Neph & TL Neph & Neph & Neph & TL Neph \\
\hline 2 & Case 15 Blend 2 & 49 & TL newlv Neph & TL newlv Neph & TL Neph & TL Neph & TL Neph & Neph & TL Neph \\
\hline 2 & Case 15 Blend 2 & 50 & TL newlv Neph & TL newlv Neph & TL newlv Neph & TL Neph & TL Neph & TL Neph & TL Neph \\
\hline 2 & Case 15 Blend 2 & 51 & TL newlv Neph & TL newlv Neph & TL newlv Neph & TL Neph & TL Neph & TL newlv Neph & TL Neph \\
\hline 2 & Case 15 Blend 2 & 52 & TL newlv Neph & TL newlv Neph & TL newlv Neph & TL Neph & TL Neph & TL newlv Neph & TL Neph \\
\hline 2 & Case 15 Blend 2 & 53 & TL newlv Neph & TL newlv Neph & TL newlv Neph & TL Neph & TL Neph & TL newlv Neph & TL newlv Neph \\
\hline 2 & Case 15 Blend 2 & 54 & TL newlv Neph & TL newlv Neph & TL newlv Neph & TL Neph & TL newlv Neph & TL newlv Neph & TL newlv Neph \\
\hline 2 & Case 15 Blend 2 & 55 & TL newlv Neph & TL newlv Neph & TL newlv Neph & TL Neph & TL newlv Neph & TL newlv Neph & TL newlv Neph \\
\hline 2 & Case 15 Blend 2 & 56 & TL newlv Neph & TL newlv Neph & TL newlv Neph & TL newlv Neph & TL newlv Neph & TL newlv Neph & TL newlv Neph \\
\hline 2 & Case 15 Blend 2 & 57 & TL newlv Neph & TL newlv Neph & TL newlv Neph & TL newlv Neph & TL newlv Neph & TL newlv Neph & TL newlv Neph \\
\hline 2 & Case 15 Blend 2 & 58 & TL newlv Neph & TL newlv Neph & TL newlv Neph & TL newlv Neph & TL newlv Neph & TL newlv Neph & TL newlv Neph \\
\hline 2 & Case 15 Blend 2 & 59 & TL newlv Neph & TL newlv Neph & TL newlv Neph & TL newlv Neph & TL newlv Neph & TL newlv Neph & TL newlv Neph \\
\hline 2 & $\begin{array}{l}\text { Case } 15 \text { Blend } 2 \\
\end{array}$ & 60 & TL newlv Neph & TL newlv Neph & TL newlv Neph & TL newlv Neph & TL newlv Neph & TL newlv Neph & TL newlv Neph \\
\hline 3 & Case 7b Batch 1 & 25 & & & & & & & \\
\hline 3 & Case 7b Batch 1 & 26 & & & & & & & \\
\hline 3 & Case 7b Batch 1 & 27 & & & & & & & \\
\hline 3 & Case 7b Batch 1 & 28 & & & & & & & \\
\hline 3 & Case 7b Batch 1 & 29 & & & & & & & \\
\hline 3 & Case $7 \mathrm{~b}$ Batch 1 & 30 & & & & & & & \\
\hline 3 & Case 7b Batch 1 & 31 & & & & & & & \\
\hline 3 & Case 7b Batch 1 & 32 & & & & & & & \\
\hline 3 & Case $7 \mathrm{~b}$ Batch 1 & 33 & & & & & & & \\
\hline 3 & Case 7b Batch 1 & 34 & & & & & & & \\
\hline 3 & Case 7b Batch 1 & 35 & & & & & & & \\
\hline 3 & Case 7b Batch 1 & 36 & & & & & & & \\
\hline 3 & Case $7 \mathrm{~b}$ Batch 1 & 37 & & & & & & & Neph \\
\hline 3 & Case $7 \mathrm{~b}$ Batch 1 & 38 & & & & & & & Neph \\
\hline 3 & Case 7b Batch 1 & 39 & & & & & & & Neph \\
\hline 3 & Case $7 \mathrm{~b}$ Batch 1 & 40 & Neph & & & & & Neph & Neph \\
\hline 3 & Case $7 \mathrm{~b}$ Batch 1 & 41 & newlv Neph & & & & Neph & Neph & Neph \\
\hline 3 & Case 7b Batch 1 & 42 & newlv Neph & Neph & Neph & Neph & Neph & Neph & Neph \\
\hline 3 & Case 7b Batch 1 & 43 & newlv Neph & Neph & Neph & Neph & Neph & Neph & Neph \\
\hline 3 & Case 7b Batch 1 & 44 & newlv Neph & Neph & Neph & Neph & Neph & Neph & Neph \\
\hline 3 & Case $7 \mathrm{~b}$ Batch 1 & 45 & newlv Neph & Neph & Neph & Neph & Neph & Neph & Neph \\
\hline 3 & Case 7b Batch 1 & 46 & newlv Neph & Neph & Neph & Neph & Neph & Neph & Neph \\
\hline 3 & Case $7 \mathrm{~b}$ Batch 1 & 47 & newlv Neph & Neph & Neph & Neph & Neph & Neph & Neph \\
\hline 3 & Case $7 \mathrm{~b}$ Batch 1 & 48 & newlv Neph & Neph & Neph & Neph & Neph & Neph & Neph \\
\hline 3 & Case 7b Batch 1 & 49 & newlv Neph & Neph & Neph & Neph & Neph & Neph & Neph \\
\hline 3 & Case $7 \mathrm{~b}$ Batch 1 & 50 & newlv Neph & newlv Neph & Neph & Neph & Neph & Neph & Neph \\
\hline 3 & Case 7b Batch 1 & 51 & TL newlv Neph & newlv Neph & Neph & Neph & Neph & Neph & Neph \\
\hline 3 & Case $7 \mathrm{~b}$ Batch 1 & 52 & TL newlv Neph & newlv Neph & newlv Neph & Neph & Neph & Neph & TL Neph \\
\hline 3 & Case $7 \mathrm{~b}$ Batch 1 & 53 & TL newlv Neph & newlv Neph & newlv Neph & Neph & Neph & newlv Neph & TL Neph \\
\hline 3 & Case 7b Batch 1 & 54 & TL newlv Neph & newlv Neph & newlv Neph & Neph & Neph & newlv Neph & TL newlv Neph \\
\hline
\end{tabular}


Exhibit A1. Results for MAR Assessments of the Nominal Stage (part 3)

Constraint not met: neph (nepheline), TL (liquidus temperature), newlv (low viscosity - new model), newhv (high viscosity - new model), and Del GP - durability

\begin{tabular}{|c|c|c|c|c|c|c|c|c|c|}
\hline & & $\mathrm{Na} 2 \mathrm{O}$ & 0.07 & 0.07 & 0.07 & 0.08 & 0.09 & 0.1 & 0.11 \\
\hline Set & Type & Sludge Loading (\%) & P2-5 & P3-3 & P3-4 & 418 & 426 & 425 & 200 \\
\hline 3 & Case $7 \mathrm{~b}$ Batch 1 & 55 & TL newlv Neph & TL newlv Neph & newlv Neph & Neph & newlv Neph & newlv Neph & TL newlv Neph \\
\hline 3 & Case $7 \mathrm{~b}$ Batch 1 & 56 & TL newlv Neph & TL newlv Neph & TL newlv Neph & TL Neph & newlv Neph & newlv Neph & TL newlv Neph \\
\hline 3 & Case 7b Batch 1 & 57 & TL newlv Neph & TL newlv Neph & TL newlv Neph & TL Neph & TL newlv Neph & TL newlv Neph & TL newlv Neph \\
\hline 3 & Case $7 \mathrm{~b}$ Batch 1 & 58 & TL newlv Neph & TL newlv Neph & TL newlv Neph & TL newlv Neph & TL newlv Neph & TL newlv Neph & TL newlv Neph \\
\hline 3 & Case 7b Batch 1 & 59 & TL newlv Neph & TL newlv Neph & TL newlv Neph & TL newlv Neph & TL newlv Neph & TL newlv Neph & TL newlv Neph \\
\hline 3 & Case $7 \mathrm{~b}$ Batch 1 & 60 & TL newlv Neph & TL newlv Neph & TL newlv Neph & TL newlv Neph & TL newlv Neph & TL newlv Neph & TL newlv Neph \\
\hline 3 & Case $7 \mathrm{~b}$ Batch 2 & 25 & & & & newhv & & & \\
\hline 3 & Case 7b Batch 2 & 26 & & & & newhy & & & \\
\hline 3 & Case $7 \mathrm{~b}$ Batch 2 & 27 & & & & newhv & & & \\
\hline 3 & Case $7 \mathrm{~b}$ Batch 2 & 28 & & & & newhy & & & \\
\hline 3 & Case $7 \mathrm{~b}$ Batch 2 & 29 & & & & & & & \\
\hline 3 & Case 7 b Batch 2 & 30 & & & & & & & \\
\hline 3 & Case 7b Batch 2 & 31 & & & & & & & \\
\hline 3 & Case $7 \mathrm{~b}$ Batch 2 & 32 & & & & & & & \\
\hline 3 & Case 7 b Batch 2 & 33 & & & & & & & \\
\hline 3 & Case $7 \mathrm{~b}$ Batch 2 & 34 & & & & & & & \\
\hline 3 & Case $7 \mathrm{~b}$ Batch 2 & 35 & & & & & & & \\
\hline 3 & Case 7b Batch 2 & 36 & & & & & & & \\
\hline 3 & Case $7 \mathrm{~b}$ Batch 2 & 37 & & & & & & & \\
\hline 3 & Case $7 \mathrm{~b}$ Batch 2 & 38 & & & & & & & Neph \\
\hline 3 & Case $7 \mathrm{~b}$ Batch 2 & 39 & & & & & & & Neph \\
\hline 3 & Case $7 \mathrm{~b}$ Batch 2 & 40 & & & & & & & Neph \\
\hline 3 & Case 7b Batch 2 & 41 & Neph & & & & & Neph & Neph \\
\hline 3 & Case $7 \mathrm{~b}$ Batch 2 & 42 & TL Neph & & & & Neph & Neph & Neph \\
\hline 3 & Case $7 \mathrm{~b}$ Batch 2 & 43 & TL Neph & Neph & Neph & Neph & Neph & Neph & TL Neph \\
\hline 3 & Case $7 \mathrm{~b}$ Batch 2 & 44 & TL Neph & Neph & Neph & Neph & Neph & Neph & TL Neph \\
\hline 3 & Case $7 \mathrm{~b}$ Batch 2 & 45 & TL Neph & Neph & Neph & Neph & Neph & Neph & TL Neph \\
\hline 3 & Case 7b Batch 2 & 46 & TL Neph & Neph & Neph & Neph & Neph & Neph & TL Neph \\
\hline 3 & Case $7 \mathrm{~b}$ Batch 2 & 47 & TL Neph & TL Neph & Neph & TL Neph & Neph & Neph & TL Neph \\
\hline 3 & Case $7 \mathrm{~b}$ Batch 2 & 48 & TL Neph & TL Neph & TL Neph & TL Neph & TL Neph & Neph & TL Neph \\
\hline 3 & Case $7 \mathrm{~b}$ Batch 2 & 49 & TL Neph & TL Neph & TL Neph & TL Neph & TL Neph & TL Neph & TL Neph \\
\hline 3 & Case 7b Batch 2 & 50 & TL Neph & TL Neph & TL Neph & TL Neph & TL Neph & TL Neph & TL Neph \\
\hline 3 & Case $7 \mathrm{~b}$ Batch 2 & 51 & TL Neph & TL Neph & TL Neph & TL Neph & TL Neph & TL Neph & TL Neph \\
\hline 3 & Case $7 \mathrm{~b}$ Batch 2 & 52 & TL Neph & TL Neph & TL Neph & TL Neph & TL Neph & TL Neph & TL Neph \\
\hline 3 & Case $7 \mathrm{~b}$ Batch 2 & 53 & TL Neph & TL Neph & TL Neph & TL Neph & TL Neph & TL Neph & TL Neph \\
\hline 3 & Case 7b Batch 2 & 54 & TL newlv Neph & TL Neph & TL Neph & TL Neph & TL Neph & TL Neph & TL Neph \\
\hline 3 & Case $7 \mathrm{~b}$ Batch 2 & 55 & TL newlv Neph & TL Neph & TL Neph & TL Neph & TL Neph & TL Neph & TL Neph \\
\hline 3 & Case $7 \mathrm{~b}$ Batch 2 & 56 & TL newlv Neph & TL Neph & TL Neph & TL Neph & TL Neph & TL Neph & TL Neph \\
\hline 3 & Case $7 \mathrm{~b}$ Batch 2 & 57 & TL newlv Neph & TL Neph & TL Neph & TL Neph & TL Neph & TL Neph & TL Neph \\
\hline 3 & Case 7b Batch 2 & 58 & TL newlv Neph & TL Neph & TL Neph & TL Neph & TL Neph & TL Neph & TL Neph \\
\hline 3 & Case 7b Batch 2 & 59 & TL newlv Neph & TL Neph & TL Neph & TL Neph & TL Neph & TL Neph & TL Neph \\
\hline 3 & Case $7 \mathrm{~b}$ Batch 2 & 60 & TL newlv Neph & TL Neph & TL Neph & TL Neph & TL Neph & TL Neph & TL Neph \\
\hline 3 & Case $7 \mathrm{~b}$ Blend 1 & 25 & & & & & & & \\
\hline 3 & Case $7 \mathrm{~b}$ Blend 1 & 26 & & & & & & & \\
\hline 3 & Case $7 \mathrm{~b}$ Blend 1 & 27 & & & & & & & \\
\hline 3 & Case $7 \mathrm{~b}$ Blend 1 & 28 & & & & & & & \\
\hline 3 & Case $7 \mathrm{~b}$ Blend 1 & 29 & & & & & & & \\
\hline 3 & Case $7 \mathrm{~b}$ Blend 1 & 30 & & & & & & & \\
\hline 3 & Case $7 \mathrm{~b}$ Blend 1 & 31 & newlv & & & & & & \\
\hline 3 & Case $7 \mathrm{~b}$ Blend 1 & 32 & newlv & & & & & & \\
\hline 3 & Case $7 \mathrm{~b}$ Blend 1 & 33 & newlv & & & & & & \\
\hline
\end{tabular}


Exhibit A1. Results for MAR Assessments of the Nominal Stage (part 3)

Constraint not met: neph (nepheline), TL (liquidus temperature), newlv (low viscosity - new model), newhv (high viscosity - new model), and Del GP - durability

\begin{tabular}{|c|c|c|c|c|c|c|c|c|c|}
\hline & & Pa2O & $\begin{array}{ll}0.07 \\
\end{array}$ & $\begin{array}{l}0.07 \\
\end{array}$ & $\begin{array}{ll}0.07 \\
\end{array}$ & $\begin{array}{ll}0.08 \\
\end{array}$ & (20.09 & $\begin{array}{ll}0.1 \\
\end{array}$ & 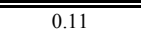 \\
\hline Set & Type & Sludge Loading (\%) & P2-5 & P3-3 & P3-4 & 418 & 426 & 425 & 200 \\
\hline 3 & Case $7 \mathrm{~b}$ Blend 1 & 34 & newlv & & & & & & \\
\hline 3 & Case $7 \mathrm{~b}$ Blend 1 & 35 & newlv & & & & & & \\
\hline 3 & Case $7 \mathrm{~b}$ Blend 1 & 36 & newlv & & & & & & \\
\hline 3 & Case $7 \mathrm{~b}$ Blend 1 & 37 & newlv & & & & & & \\
\hline 3 & Case $7 \mathrm{~b}$ Blend 1 & 38 & newlv & & & & & & \\
\hline 3 & Case $7 \mathrm{~b}$ Blend 1 & 39 & newlv & newlv & & & & & \\
\hline 3 & Case $7 \mathrm{~b}$ Blend 1 & 40 & newlv & newlv & & & & & \\
\hline 3 & Case $7 \mathrm{~b}$ Blend 1 & 41 & newlv & newlv & newlv & & & & \\
\hline 3 & Case $7 \mathrm{~b}$ Blend 1 & 42 & newlv & newlv & newlv & & & newly & \\
\hline 3 & Case $7 \mathrm{~b}$ Blend 1 & 43 & newlv & newlv & newlv & & & newlv & Neph \\
\hline 3 & Case $7 \mathrm{~b}$ Blend 1 & 44 & newlv & newlv & newlv & & & newlv & newlv Neph \\
\hline$\frac{5}{3}$ & Case $7 \mathrm{~b}$ Blend 1 & 45 & TL newlv & newlv & newlv & & newlv & newly & newlv Neph \\
\hline 3 & Case $7 \mathrm{~b}$ Blend 1 & 46 & TL newlv Neph & newlv & newlv & & newlv & newlv Neph & TL newlv Neph \\
\hline 3 & Case $7 \mathrm{~b}$ Blend 1 & 47 & TL newlv Neph & newlv & newlv & newlv & newlv Neph & newlv Neph & TL newlv Neph \\
\hline$\frac{3}{3}$ & Case $7 \mathrm{~b}$ Blend 1 & 48 & TL newlv Neph & newlv Neph & newlv Neph & newlv Neph & newlv Neph & newlv Neph & TL newlv Neph \\
\hline 3 & Case $7 \mathrm{~b}$ Blend 1 & 49 & TL newlv Neph & TL newlv Neph & newlv Neph & TL newlv Neph & newlv Neph & newlv Neph & TL newlv Neph \\
\hline 3 & Case $7 \mathrm{~b}$ Blend 1 & 50 & TL newlv Neph & TL newlv Neph & TL newlv Neph & TL newlv Neph & TL newlv Neph & newlv Neph & TL newlv Neph \\
\hline 3 & Case $7 \mathrm{~b}$ Blend 1 & 51 & TL newlv Neph & TL newlv Neph & TL newlv Neph & TL newlv Neph & TL newlv Neph & TL newlv Neph & TL newlv Neph \\
\hline 3 & Case $7 \mathrm{~b}$ Blend 1 & 52 & TL newlv Neph & TL newlv Neph & TL newlv Neph & TL newlv Neph & TL newlv Neph & TL newlv Neph & TL newlv Neph \\
\hline 3 & Case $7 \mathrm{~b}$ Blend 1 & 53 & TL newlv Neph & TL newlv Neph & TL newlv Neph & TL newlv Neph & TL newlv Neph & TL newlv Neph & TL newlv Neph \\
\hline 3 & Case $7 \mathrm{~b}$ Blend 1 & 54 & TL newlv Neph & TL newlv Neph & TL newlv Neph & TL newlv Neph & TL newlv Neph & TL newlv Neph & TL newlv Neph \\
\hline 3 & Case $7 \mathrm{~b}$ Blend 1 & 55 & TL newlv Neph & TL newlv Neph & TL newlv Neph & TL newlv Neph & TL newlv Neph & TL newlv Neph & TL newlv Neph \\
\hline$\frac{3}{3}$ & Case $7 \mathrm{~b}$ Blend 1 & 56 & TL newlv Neph & TL newlv Neph & TL newlv Neph & TL newlv Neph & TL newlv Neph & TL newlv Neph & TL newlv Neph \\
\hline 3 & Case $7 \mathrm{~b}$ Blend 1 & 57 & TL newlv Neph & TL newlv Neph & TL newlv Neph & TL newlv Neph & TL newlv Neph & TL newlv Neph & TL newlv Neph \\
\hline 3 & Case $7 \mathrm{~b}$ Blend 1 & 58 & TL newlv Neph & TL newlv Neph & TL newlv Neph & TL newlv Neph & TL newlv Neph & TL newlv Neph & TL newlv Neph \\
\hline$\frac{3}{3}$ & Case $7 \mathrm{~b}$ Blend 1 & 59 & TL newlv Neph & TL newlv Neph & TL newlv Neph & TL newlv Neph & TL newlv Neph & TL newlv Neph & TL newlv Neph \\
\hline 3 & Case $7 \mathrm{~b}$ Blend 1 & 60 & TL newlv Neph & TL newlv Neph & TL newlv Neph & TL newlv Neph & TL newlv Neph & TL newlv Neph & TL newlv Neph \\
\hline 3 & Case $7 \mathrm{~b}$ Blend 2 & 25 & & & & & & & \\
\hline 3 & Case $7 \mathrm{~b}$ Blend 2 & 26 & & & & & & & \\
\hline 3 & Case $7 \mathrm{~b}$ Blend 2 & 27 & & & & & & & \\
\hline 3 & Case $7 \mathrm{~b}$ Blend 2 & 28 & & & & & & & \\
\hline 3 & Case $7 \mathrm{~b}$ Blend 2 & 29 & & & & & & & \\
\hline 3 & Case $7 \mathrm{~b}$ Blend 2 & 30 & & & & & & & \\
\hline 3 & Case $7 \mathrm{~b}$ Blend 2 & 31 & & & & & & & \\
\hline$\frac{3}{3}$ & Case $7 \mathrm{~b}$ Blend 2 & $\frac{31}{32}$ & & & & & & & \\
\hline 3 & Case $7 \mathrm{~b}$ Blend 2 & 33 & & & & & & & \\
\hline 3 & Case $7 \mathrm{~b}$ Blend 2 & 34 & & & & & & & \\
\hline 3 & Case $7 \mathrm{~b}$ Blend 2 & 35 & & & & & & & \\
\hline 3 & Case $7 \mathrm{~b}$ Blend 2 & 36 & & & & & & & \\
\hline$\frac{3}{3}$ & Case $7 \mathrm{~b}$ Blend 2 & 37 & & & & & & & \\
\hline 3 & Case $7 \mathrm{~b}$ Blend 2 & 38 & & & & & & & \\
\hline 3 & Case $7 \mathrm{~b}$ Blend 2 & 39 & & & & & & & Neph \\
\hline 3 & Case $7 \mathrm{~b}$ Blend 2 & 40 & & & & & & & Neph \\
\hline 3 & Case $7 \mathrm{~b}$ Blend 2 & 41 & & & & & & & Neph \\
\hline 3 & Case $7 \mathrm{~b}$ Blend 2 & 42 & Neph & & & & & Neph & Neph \\
\hline 3 & Case $7 \mathrm{~b}$ Blend 2 & 43 & TL Neph & & & & Neph & Neph & Neph \\
\hline 3 & Case $7 \mathrm{~b}$ Blend 2 & 44 & TL Neph & Neph & Neph & Neph & Neph & Neph & TL Neph \\
\hline$\frac{3}{3}$ & Case $7 \mathrm{~b}$ Blend 2 & 45 & TL newlv Neph & Neph & Neph & Neph & Neph & Neph & TL Neph \\
\hline 3 & Case $7 \mathrm{~b}$ Blend 2 & 46 & TL newlv Neph & Neph & Neph & Neph & Neph & Neph & TL Neph \\
\hline 3 & Case $7 \mathrm{~b}$ Blend 2 & 47 & TL newlv Neph & Neph & Neph & Neph & Neph & Neph & TL Neph \\
\hline 3 & Case $7 \mathrm{~b}$ Blend 2 & 48 & TL newlv Neph & TL Neph & Neph & TL Neph & Neph & Neph & TL Neph \\
\hline
\end{tabular}




\section{Exhibit A1. Results for MAR Assessments of the Nominal Stage (part 3)}

Constraint not met: neph (nepheline), TL (liquidus temperature), newlv (low viscosity - new model), newhv (high viscosity - new model), and Del GP - durability

\begin{tabular}{|c|c|c|c|c|c|c|c|c|c|}
\hline & & $\mathrm{Na} 2 \mathrm{O}$ & 0.07 & 0.07 & 0.07 & 0.08 & 0.09 & 0.1 & 0.11 \\
\hline Set & Type & $\begin{array}{l}\text { Sludge Loading }(\%) \\
\end{array}$ & P2-5 & $\begin{array}{l}\text { P3-3 } \\
\end{array}$ & $\begin{array}{l}\text { P3-4 } \\
\end{array}$ & 418 & 426 & 425 & 200 \\
\hline 3 & Case $7 \mathrm{~b}$ Blend 2 & 49 & TL newlv Neph & TL Neph & TL Neph & TL Neph & TL Neph & Neph & TL Neph \\
\hline 3 & Case $7 \mathrm{~b}$ Blend 2 & 50 & TL newlv Neph & TL Neph & TL Neph & TL Neph & TL Neph & TL Neph & TL Neph \\
\hline 3 & Case $7 \mathrm{~b}$ Blend 2 & 51 & TL newlv Neph & TL Neph & TL Neph & TL Neph & TL Neph & TL Neph & TL Neph \\
\hline 3 & Case $7 \mathrm{~b}$ Blend 2 & 52 & TL newlv Neph & TL Neph & TL Neph & TL Neph & TL Neph & TL Neph & TL Neph \\
\hline 3 & Case $7 \mathrm{~b}$ Blend 2 & 53 & TL newlv Neph & TL Neph & TL Neph & TL Neph & TL Neph & TL Neph & TL Neph \\
\hline 3 & Case $7 \mathrm{~b}$ Blend 2 & 54 & TL newlv Neph & TL Neph & TL Neph & TL Neph & TL Neph & TL Neph & TL Neph \\
\hline 3 & Case $7 \mathrm{~b}$ Blend 2 & 55 & TL newlv Neph & TL newlv Neph & TL Neph & TL Neph & TL Neph & TL Neph & TL Neph \\
\hline 3 & Case $7 \mathrm{~b}$ Blend 2 & 56 & TL newlv Neph & TL newlv Neph & TL newlv Neph & TL Neph & TL Neph & TL Neph & TL Neph \\
\hline 3 & Case $7 \mathrm{~b}$ Blend 2 & 57 & TL newlv Neph & TL newlv Neph & TL newlv Neph & TL Neph & TL Neph & TL newlv Neph & TL Neph \\
\hline 3 & Case $7 \mathrm{~b}$ Blend 2 & 58 & TL newly Neph & TL newlv Neph & TL newlv Neph & TL Neph & TL Neph & TL newlv Neph & TL Neph \\
\hline 3 & Case $7 \mathrm{~b}$ Blend 2 & 59 & TL newlv Neph & TL newlv Neph & TL newlv Neph & TL Neph & TL Neph & TL newlv Neph & TL newlv Neph \\
\hline 3 & Case $7 \mathrm{~b}$ Blend 2 & 60 & TL newlv Neph & TL newlv Neph & TL newlv Neph & TL Neph & TL newlv Neph & TL newlv Neph & TL newlv Neph \\
\hline
\end{tabular}


Exhibit A1. Results for MAR Assessments of the Nominal Stage (part 4)

Constraint not met: neph (nepheline), TL (liquidus temperature), newlv (low viscosity - new model), newhv (high viscosity - new model), and Del GP - durability

\begin{tabular}{|c|c|c|c|c|c|c|c|}
\hline & & $\mathrm{Na} 2 \mathrm{O}$ & 0.11 & 0.12 & 0.13 & 0.14 & 0.15 \\
\hline Set & Type & $\begin{array}{l}\text { Sludge Loading (\%) } \\
\end{array}$ & 417 & 320 & 431 & $\mathrm{Y} 1-1$ & $\mathrm{X} 1-1$ \\
\hline 1 & Case 15B - Batch 1 & 25 & & & & & \\
\hline 1 & Case 15B - Batch 1 & 26 & & & & & \\
\hline 1 & Case 15B - Batch 1 & 27 & & & & & \\
\hline 1 & Case 15B - Batch 1 & 28 & & & & & \\
\hline 1 & Case 15B - Batch 1 & 29 & & & & & \\
\hline 1 & Case 15B - Batch 1 & 30 & & & & & \\
\hline 1 & Case 15B - Batch 1 & 31 & & & & & \\
\hline 1 & Case 15B - Batch 1 & 32 & & & & & Neph \\
\hline 1 & Case 15B - Batch 1 & 33 & & & & Neph & Neph \\
\hline 1 & Case 15B - Batch 1 & 34 & & & Neph & Neph & Neph \\
\hline 1 & Case 15B - Batch 1 & 35 & & Neph & Neph & Neph & Neph \\
\hline 1 & Case 15B - Batch 1 & 36 & Neph & Neph & Neph & Neph & Neph \\
\hline 1 & Case 15B - Batch 1 & 37 & Neph & Neph & Neph & Neph & Neph \\
\hline 1 & Case 15B - Batch 1 & 38 & Neph & Neph & Neph & Neph & Neph \\
\hline 1 & Case 15B - Batch 1 & 39 & Neph & Neph & Neph & Neph & Neph \\
\hline 1 & Case 15B - Batch 1 & 40 & Neph & Neph & Neph & Neph & Neph \\
\hline 1 & Case 15B - Batch 1 & 41 & Neph & Neph & Neph & Neph & Neph \\
\hline 1 & Case 15B - Batch 1 & 42 & Neph & Neph & Neph & Neph & Neph \\
\hline 1 & Case 15B - Batch 1 & 43 & Neph & Neph & Neph & Neph & Neph \\
\hline 1 & Case 15B - Batch 1 & 44 & Neph & Neph & Neph & Neph & Neph \\
\hline 1 & Case 15B - Batch 1 & 45 & Neph & Neph & Neph & Neph & Neph \\
\hline 1 & Case 15B - Batch 1 & 46 & Neph & Neph & Neph & Neph & Neph \\
\hline 1 & Case 15B - Batch 1 & 47 & Neph & Neph & Neph & Neph & Neph \\
\hline 1 & Case 15B - Batch 1 & 48 & Neph & Neph & Neph & Neph & Neph \\
\hline 1 & Case 15B - Batch 1 & 49 & Neph & Neph & Neph & Neph & Neph \\
\hline 1 & Case 15B - Batch 1 & 50 & Neph & Neph & Neph & Neph & Neph \\
\hline 1 & Case 15B - Batch 1 & 51 & Neph & Neph & Neph & Neph & Neph \\
\hline 1 & Case 15B - Batch 1 & 52 & Neph & Neph & Neph & Neph & Neph \\
\hline 1 & Case 15B - Batch 1 & 53 & Neph & Neph & Neph & Neph & Neph \\
\hline 1 & Case 15B - Batch 1 & 54 & Neph & Neph & Neph & Neph & Neph \\
\hline 1 & Case 15B - Batch 1 & 55 & Neph & Neph & Neph & Neph & Neph \\
\hline 1 & Case 15B - Batch 1 & 56 & Neph & Neph & Neph & Neph & Neph \\
\hline 1 & Case 15B - Batch 1 & 57 & Neph & Neph & Neph & Neph & Neph \\
\hline 1 & Case 15B - Batch 1 & 58 & Neph & Neph & Neph & Neph & Neph \\
\hline 1 & Case 15B - Batch 1 & 59 & Neph & Neph & Neph & Neph & Neph \\
\hline 1 & Case 15B - Batch 1 & 60 & Neph & Neph & Neph & Neph & Neph \\
\hline 1 & Case 15B - Batch 2 & 25 & & & & & \\
\hline 1 & Case 15B - Batch 2 & 26 & & & & & \\
\hline 1 & Case 15B - Batch 2 & 27 & & & & & \\
\hline 1 & Case 15B - Batch 2 & 28 & & & & & \\
\hline 1 & Case 15B - Batch 2 & 29 & & & & & \\
\hline 1 & Case 15B - Batch 2 & 30 & & & & & \\
\hline 1 & Case 15B - Batch 2 & 31 & & & & & \\
\hline 1 & Case 15B - Batch 2 & 32 & & & & & \\
\hline 1 & Case 15B - Batch 2 & 33 & & & & & \\
\hline 1 & Case 15B - Batch 2 & 34 & & & & & \\
\hline 1 & Case 15B - Batch 2 & 35 & & & & & \\
\hline 1 & Case 15B - Batch 2 & 36 & & & & & Neph \\
\hline 1 & Case 15B - Batch 2 & 37 & & & & Neph & Neph \\
\hline 1 & Case 15B - Batch 2 & 38 & & & Neph & Neph & Neph \\
\hline 1 & Case 15B - Batch 2 & 39 & & & Neph & Neph & Neph \\
\hline
\end{tabular}


Exhibit A1. Results for MAR Assessments of the Nominal Stage (part 4)

Constraint not met: neph (nepheline), TL (liquidus temperature), newlv (low viscosity - new model), newhv (high viscosity - new model), and Del GP - durability

\begin{tabular}{|c|c|c|c|c|c|c|c|}
\hline & & $\mathrm{Na} 2 \mathrm{O}$ & 0.11 & 0.12 & 0.13 & 0.14 & 0.15 \\
\hline Set & Type & Sludge Loading (\%) & 417 & 320 & 431 & $\mathrm{Y} 1-1$ & $\mathrm{X1-1}$ \\
\hline 1 & Case 15B - Batch 2 & 40 & & Neph & Neph & Neph & Neph \\
\hline 1 & Case 15B - Batch 2 & 41 & Neph & Neph & Neph & Neph & Neph \\
\hline 1 & Case 15B - Batch 2 & 42 & Neph & Neph & Neph & Neph & Neph \\
\hline 1 & Case 15B - Batch 2 & 43 & Neph & Neph & Neph & Neph & Neph \\
\hline 1 & Case 15B - Batch 2 & 44 & Neph & Neph & Neph & Neph & Neph \\
\hline 1 & Case 15B - Batch 2 & 45 & Neph & Neph & Neph & Neph & Neph \\
\hline 1 & Case 15B - Batch 2 & 46 & TL Neph & Neph & Neph & Neph & Neph \\
\hline 1 & Case 15B - Batch 2 & 47 & TL Neph & TL Neph & Neph & Neph & Neph \\
\hline 1 & Case 15B - Batch 2 & 48 & TL Neph & TL Neph & TL Neph & Neph & Neph \\
\hline 1 & Case 15B - Batch 2 & 49 & TL Neph & TL Neph & TL Neph & TL Neph & Neph \\
\hline 1 & Case 15B - Batch 2 & 50 & TL Neph & TL Neph & TL Neph & TL Neph & TL newlv Neph \\
\hline 1 & Case 15B - Batch 2 & 51 & TL Neph & TL Neph & TL Neph & TL Neph & TL newlv Neph \\
\hline 1 & Case 15B - Batch 2 & 52 & TL Neph & TL Neph & TL Neph & TL Neph & TL newlv Neph \\
\hline 1 & Case 15B - Batch 2 & 53 & TL Neph & TL Neph & TL Neph & TL Neph & TL newlv Neph \\
\hline 1 & Case 15B - Batch 2 & 54 & TL Neph & TL Neph & TL Neph & TL Neph & TL newlv Neph \\
\hline 1 & Case 15B - Batch 2 & 55 & TL Neph & TL Neph & TL Neph & TL newlv Neph & TL newlv Neph \\
\hline 1 & Case 15B - Batch 2 & 56 & TL Neph & TL Neph & TL Neph & TL newlv Neph & TL newlv Neph \\
\hline 1 & Case 15B - Batch 2 & 57 & TL Neph & TL Neph & TL Neph & TL newlv Neph & TL newlv Neph \\
\hline 1 & Case 15B - Batch 2 & 58 & TL Neph & TL Neph & TL Neph & TL newlv Neph & TL newlv Neph \\
\hline 1 & Case 15B - Batch 2 & 59 & TL Neph & TL Neph & TL Neph & TL newlv Neph & TL newlv Neph \\
\hline 1 & Case 15B - Batch 2 & 60 & TL Neph & TL Neph & TL newlv Neph & TL newlv Neph & TL newlv Neph \\
\hline 1 & Case 15B - Blend 1 & 25 & & & Del Gp & Del Gp & Del Gp newlv \\
\hline 1 & Case 15B - Blend 1 & 26 & & & Del Gp & Del Gp & Del Gp newlv \\
\hline 1 & Case 15B - Blend 1 & 27 & & & Del Gp & Del Gp & Del Gp newlv \\
\hline 1 & Case 15B - Blend 1 & 28 & & & Del Gp & Del Gp & Del Gp newlv \\
\hline 1 & Case 15B - Blend 1 & 29 & & & Del Gp & Del Gp newlv & Del Gp newlv \\
\hline 1 & Case 15B - Blend 1 & 30 & & & Del Gp & Del Gp newlv & Del Gp newlv \\
\hline 1 & Case 15B - Blend 1 & 31 & & & Del Gp & Del Gp newlv & Del Gp newlv \\
\hline 1 & Case $15 \mathrm{~B}-$ Blend 1 & 32 & & & Del Gp newlv & Del Gp newlv & Del Gp newlv \\
\hline 1 & Case 15B - Blend 1 & 33 & & & Del Gp newlv & Del Gp newlv & Del Gp newlv \\
\hline 1 & Case 15B - Blend 1 & 34 & & & Del Gp newlv & Del Gp newlv & Del Gp newlv \\
\hline 1 & Case 15B - Blend 1 & 35 & & & Del Gp newlv & Del Gp newlv & Del Gp newlv \\
\hline 1 & Case 15B - Blend 1 & 36 & & newlv & Del Gp newlv & Del Gp newlv & Del Gp newlv \\
\hline 1 & Case 15B - Blend 1 & 37 & & newlv & Del Gp newlv & Del Gp newlv & Del Gp newlv \\
\hline 1 & Case $15 \mathrm{~B}$ - Blend 1 & 38 & & newly & Del Gp newlv & Del Gp newlv & Del Gp newlv \\
\hline 1 & Case $15 \mathrm{~B}-$ Blend 1 & 39 & newlv & newlv & Del Gp newlv & Del Gp newlv & Del Gp newlv \\
\hline 1 & Case 15B - Blend 1 & 40 & newlv & newlv & Del Gp newlv & Del Gp newlv & Del Gp newlv \\
\hline 1 & Case 15B - Blend 1 & 41 & newlv & newlv & Del Gp newlv & Del Gp newlv & Del Gp newlv \\
\hline 1 & Case 15B - Blend 1 & 42 & newlv & newlv & Del Gp newlv & $\begin{array}{l}\text { Del Gp newlv } \\
\end{array}$ & Del Gp newlv Nepl \\
\hline 1 & Case $15 \mathrm{~B}-$ Blend 1 & 43 & newlv & newlv & Del Gp newlv & Del Gp newlv Neph & Del Gp newlv Nepl \\
\hline 1 & Case 15B - Blend 1 & 44 & newlv & newlv & newlv Neph & Del Gp newlv Neph & Del Gp newlv Nepl \\
\hline 1 & $\begin{array}{l}\text { Case } 15 \mathrm{~B} \text { - Blend } 1 \\
\end{array}$ & 45 & newlv & newlv & newlv Neph & Del Gp newlv Neph & Del Gp newlv Nepl \\
\hline 1 & Case $15 \mathrm{~B}$ - Blend 1 & 46 & newlv & newlv Neph & newlv Neph & Del Gp newlv Neph & Del Gp newlv Nepl \\
\hline 1 & Case 15B - Blend 1 & 47 & newlv Neph & newlv Neph & newlv Neph & Del Gp newlv Neph & Del Gp newlv Nepl \\
\hline 1 & Case 15B - Blend 1 & 48 & newlv Neph & newlv Neph & newlv Neph & Del Gp newlv Neph & Del Gp newlv Nepl \\
\hline 1 & Case $15 \mathrm{~B}$ - Blend 1 & 49 & newlv Neph & newlv Neph & newlv Neph & Del Gp newlv Neph & Del Gp newlv Nepl \\
\hline 1 & Case $15 \mathrm{~B}-$ Blend 1 & 50 & newlv Neph & newlv Neph & newlv Neph & Del Gp newlv Neph & Del Gp newlv Nepl \\
\hline 1 & Case 15B - Blend 1 & 51 & TL newlv Neph & newlv Neph & newlv Neph & Del Gp newlv Neph & Del Gp newlv Nepl \\
\hline 1 & Case 15B - Blend 1 & 52 & TL newlv Neph & TL newlv Neph & newlv Neph & Del Gp newlv Neph & Del Gp newlv Nepl \\
\hline 1 & Case 15B - Blend 1 & 53 & TL newlv Neph & TL newlv Neph & TL newlv Neph & Del Gp newlv Neph & Del Gp newlv Nepl \\
\hline 1 & Case $15 \mathrm{~B}-$ Blend 1 & 54 & TL newlv Neph & TL newlv Neph & TL newlv Neph & Del Gp TL newlv Neph & Del Gp newlv Nepl \\
\hline
\end{tabular}


Exhibit A1. Results for MAR Assessments of the Nominal Stage (part 4)

Constraint not met: neph (nepheline), TL (liquidus temperature), newlv (low viscosity - new model), newhv (high viscosity - new model), and Del GP - durability

\begin{tabular}{|c|c|c|c|c|c|c|c|}
\hline & & Na2O & 0.11 & 0.12 & 0.13 & 0.14 & 0.15 \\
\hline Set & $\begin{array}{c}\text { Type } \\
\end{array}$ & Sludge Loading (\%) & 417 & 320 & 431 & Y1-1 & $\mathrm{X} 1-1$ \\
\hline 1 & Case 15B - Blend 1 & 55 & TL newlv Neph & TL newlv Neph & TL newlv Neph & Del Gp TL newlv Neph & Del Gp TL newlv Neph \\
\hline 1 & $\begin{array}{l}\text { Case 15B - Blend } 1 \\
\end{array}$ & 56 & TL newlv Neph & TL newlv Neph & TL newlv Neph & Del Gp TL newlv Neph & Del Gp TL newlv Neph \\
\hline 1 & Case 15B - Blend 1 & 57 & TL newlv Neph & TL newlv Neph & TL newlv Neph & Del Gp TL newlv Neph & Del Gp TL newlv Neph \\
\hline 1 & Case 15B - Blend 1 & 58 & TL newlv Neph & TL newlv Neph & TL newlv Neph & Del Gp TL newlv Neph & Del Gp TL newlv Neph \\
\hline 1 & Case 15B - Blend 1 & 59 & TL newlv Neph & TL newly Neph & TL newlv Neph & Del Gp TL newlv Neph & Del Gp TL newlv Neph \\
\hline 1 & Case 15B - Blend 1 & 60 & TL newlv Neph & TL newlv Neph & TL newlv Neph & Del Gp TL newlv Neph & Del Gp TL newlv Neph \\
\hline 1 & Case 15B - Blend 2 & 25 & & & & & Del Gp \\
\hline 1 & Case 15B - Blend 2 & 26 & & & & & Del Gp \\
\hline 1 & Case 15B - Blend 2 & 27 & & & & & Del Gp \\
\hline 1 & $\begin{array}{l}\text { Case } 15 \mathrm{~B} \text { - Blend } 2 \\
\end{array}$ & 28 & & & & & Del Gp \\
\hline 1 & $\begin{array}{l}\text { Case } 15 \mathrm{~B} \text { - Blend } 2 \\
\end{array}$ & 29 & & & & & Del Gp \\
\hline 1 & Case 15B - Blend 2 & 30 & & & & & \\
\hline 1 & Case 15B - Blend 2 & 31 & & & & & \\
\hline 1 & Case 15B - Blend 2 & 32 & & & & & \\
\hline 1 & Case $15 \mathrm{~B}-$ Blend 2 & 33 & & & & & \\
\hline 1 & Case 15B - Blend 2 & 34 & & & & & \\
\hline 1 & Case 15B - Blend 2 & 35 & & & & & \\
\hline 1 & Case 15B - Blend 2 & 36 & & & & & \\
\hline 1 & Case 15B - Blend 2 & 37 & & & & & Neph \\
\hline 1 & Case 15B - Blend 2 & 38 & & & & Neph & Neph \\
\hline 1 & $\begin{array}{l}\text { Case } 15 \mathrm{~B} \text { - Blend } 2 \\
\end{array}$ & 39 & & & Neph & Neph & Neph \\
\hline 1 & Case $15 \mathrm{~B}$ - Blend 2 & 40 & & Neph & Neph & Neph & Neph \\
\hline 1 & Case 15B - Blend 2 & 41 & & Neph & Neph & Neph & newlv Neph \\
\hline 1 & Case 15B - Blend 2 & 42 & Neph & Neph & Neph & Neph & newlv Neph \\
\hline 1 & Case 15B - Blend 2 & 43 & Neph & Neph & Neph & Neph & newlv Neph \\
\hline 1 & Case 15B - Blend 2 & 44 & Neph & Neph & Neph & Neph & newlv Neph \\
\hline 1 & Case 15B - Blend 2 & 45 & Neph & Neph & Neph & Neph & newlv Neph \\
\hline 1 & Case 15B - Blend 2 & 46 & Neph & Neph & Neph & newlv Neph & newlv Neph \\
\hline 1 & Case 15B - Blend 2 & 47 & TL Neph & Neph & Neph & newlv Neph & newlv Neph \\
\hline 1 & Case 15B - Blend 2 & 48 & TL Neph & TL Neph & Neph & newlv Neph & newlv Neph \\
\hline 1 & Case 15B - Blend 2 & 49 & TL Neph & TL Neph & TL Neph & newlv Neph & newlv Neph \\
\hline 1 & Case 15B - Blend 2 & 50 & TL Neph & TL Neph & TL newlv Neph & TL newlv Neph & newlv Neph \\
\hline 1 & $\begin{array}{l}\text { Case 15B - Blend } 2 \\
\end{array}$ & 51 & TL Neph & TL Neph & TL newlv Neph & TL newlv Neph & TL newlv Neph \\
\hline 1 & Case 15B - Blend 2 & 52 & TL Neph & TL Neph & TL newlv Neph & TL newlv Neph & TL newlv Neph \\
\hline 1 & $\begin{array}{l}\text { Case 15B - Blend } 2 \\
\end{array}$ & 53 & TL Neph & TL Neph & TL newlv Neph & TL newlv Neph & TL newlv Neph \\
\hline 1 & Case 15B - Blend 2 & 54 & TL Neph & TL newlv Neph & TL newlv Neph & TL newlv Neph & TL newlv Neph \\
\hline 1 & Case 15B - Blend 2 & 55 & TL Neph & TL newlv Neph & TL newlv Neph & TL newlv Neph & TL newlv Neph \\
\hline 1 & Case 15B - Blend 2 & 56 & TL Neph & TL newlv Neph & TL newlv Neph & TL newlv Neph & TL newlv Neph \\
\hline 1 & Case 15B - Blend 2 & 57 & TL Neph & TL newlv Neph & TL newlv Neph & TL newlv Neph & TL newlv Neph \\
\hline 1 & Case $15 \mathrm{~B}-$ Blend 2 & 58 & TL newlv Neph & TL newlv Neph & TL newlv Neph & TL newlv Neph & TL newlv Neph \\
\hline 1 & Case 15B - Blend 2 & 59 & TL newlv Neph & TL newlv Neph & TL newlv Neph & TL newlv Neph & TL newlv Neph \\
\hline 1 & Case 15B - Blend 2 & 60 & TL newlv Neph & TL newlv Neph & TL newlv Neph & TL newlv Neph & TL newlv Neph \\
\hline 1 & Case 16 - Batch 1 & 25 & Del Gp Al2O3 R2O & Del Gp newlv Al2O3 R2O & Del Gp newlv Al203 R2O & Del Gp newlv Al2O3 R2O & Del Gp newlv Al2O3 R2O \\
\hline 1 & Case 16 - Batch 1 & 26 & Del Gp newlv Al2O3 R2O & Del Gp newlv Al2O3 R2O & Del Gp newlv Al2O3 R2O & Del Gp newlv Al2O3 R2O & Del Gp newlv Al2O3 R2O \\
\hline 1 & Case 16 - Batch 1 & 27 & Del Gp newlv Al2O3 R2O & Del Gp newlv Al2O3 R2O & Del Gp newlv Al203 R2O & Del Gp newlv Al2O3 R2O & Del Gp newlv Al2O3 R2O \\
\hline 1 & Case 16 - Batch 1 & 28 & Del Gp newlv Al2O3 R2O & Del Gp newlv Al2O3 R2O & Del Gp newlv Al2O3 R2O & Del Gp newlv Al2O3 R2O & Del Gp newlv Al2O3 R2O \\
\hline 1 & Case $16-$ Batch 1 & 29 & $\begin{array}{l}\text { Del Gp newlv Al2O3 R2O } \\
\end{array}$ & Del Gp newlv Al2O3 R2O & $\begin{array}{l}\text { Del Gp newlv Al2O3 R2O } \\
\end{array}$ & $\begin{array}{l}\text { Del Gp newlv Al2O3 R2O } \\
\end{array}$ & Del Gp newlv Al2O3 R2O \\
\hline 1 & Case 16 - Batch 1 & 30 & Del Gp newlv Al2O3 R2O & Del Gp newlv Al2O3 R2O & Del Gp newlv Al2O3 R2O & Del Gp newlv Al2O3 R2O & Del Gp newlv Al2O3 R2O \\
\hline 1 & Case 16 - Batch 1 & 31 & Del Gp newlv Al2O3 R2O & Del Gp newlv Al2O3 R2O & Del Gp newlv Al2O3 R2O & Del Gp newlv Al2O3 R2O & Del Gp newlv Al2O3 R2O \\
\hline 1 & Case 16 - Batch 1 & 32 & Del Gp newlv Al2O3 R2O & Del Gp newlv Al2O3 R2O & Del Gp newlv $\mathrm{Al} 2 \mathrm{O} 3 \mathrm{R} 2 \mathrm{O}$ & Del Gp newlv $\mathrm{Al} 2 \mathrm{O} 3 \mathrm{R} 2 \mathrm{O}$ & Del Gp newlv Al2O3 R2O \\
\hline 1 & Case 16 - Batch 1 & 33 & Del Gp newlv Al2O3 R2O & Del Gp newlv Al2O3 R2O & Del Gp newlv Al2O3 R2O & Del Gp newlv Al2O3 R2O & Del Gp newlv Al2O3 R2O \\
\hline
\end{tabular}


Exhibit A1. Results for MAR Assessments of the Nominal Stage (part 4)

Constraint not met: neph (nepheline), TL (liquidus temperature), newlv (low viscosity - new model), newhv (high viscosity - new model), and Del GP - durability

\begin{tabular}{|c|c|c|c|c|c|c|c|}
\hline & & Na2O & 0.11 & 0.12 & 0.13 & 0.14 & 0.15 \\
\hline Set & Type & Sludge Loading (\%) & 417 & 320 & 431 & Y1-1 & $\mathrm{X} 1-1$ \\
\hline 1 & Case 16 - Batch 1 & 34 & Del Gp newlv Al2O3 R2O & Del Gp newlv Al2O3 R2O & Del Gp newlv Al2O3 R2O & Del Gp newlv Al2O3 R2O & Del Gp newlv Al2O3 R2O \\
\hline 1 & Case 16 - Batch 1 & 35 & Del Gp newlv Al2O3 R2O & Del Gp newlv Al2O3 R2O & Del Gp newlv Al2O3 R2O & Del Gp newlv Al2O3 R2O & Del Gp newlv Al2O3 R2O \\
\hline 1 & Case 16 - Batch 1 & 36 & Del Gp newlv Al2O3 R2O & Del Gp newlv Al2O3 R2O & Del Gp newlv Al2O3 R2O & Del Gp newlv Al2O3 R2O & Del Gp newlv Al2O3 R2O \\
\hline 1 & Case 16 - Batch 1 & 37 & Del Gp newlv Al2O3 R2O & Del Gp newlv Al2O3 R2O & Del Gp newlv Al2O3 R2O & Del Gp newlv Al2O3 R2O & Del Gp newlv Al2O3 R2O \\
\hline 1 & Case 16 - Batch 1 & 38 & Del Gp newlv Al2O3 R2O & Del Gp newlv Al2O3 R2O & Del Gp newlv Al2O3 R2O & Del Gp newlv Al2O3 R2O & Del Gp newlv Al2O3 R2O \\
\hline 1 & Case 16 - Batch 1 & 39 & Del Gp newlv Al2O3 R2O & Del Gp newlv Al2O3 R2O & Del Gp newlv Al2O3 R2O & Del Gp newlv Al2O3 R2O & $\begin{array}{l}\text { Del Gp newlv Al2O3 R2O } \\
\end{array}$ \\
\hline 1 & Case 16 - Batch 1 & 40 & Del Gp newlv Al2O3 R2O & Del Gp newlv Al2O3 R2O & Del Gp newlv Al2O3 R2O & Del Gp newlv Al2O3 R2O & Del Gp newlv Al2O3 R2O \\
\hline 1 & Case 16 - Batch 1 & 41 & Del Gp newlv Al2O3 R2O & Del Gp newlv Al2O3 R2O & Del Gp newlv Al2O3 R2O & Del Gp newlv Al2O3 R2O & Del Gp newlv Al2O3 R2O \\
\hline 1 & Case 16 - Batch 1 & 42 & Del Gp newlv Al2O3 R2O & Del Gp newlv Al2O3 R2O & Del Gp newlv Al2O3 R2O & Del Gp newlv Al2O3 R2O & Del Gp newlv Al2O3 R2O \\
\hline 1 & Case 16 - Batch 1 & 43 & Del Gp newlv Al2O3 R2O & Del Gp newlv Al2O3 R2O & Del Gp newlv Al2O3 R2O & Del Gp newlv Al2O3 R2O & Del Gp newlv Al2O3 R2O Neph \\
\hline 1 & Case 16 - Batch 1 & 44 & Del Gp newlv Al2O3 R2O & Del Gp newlv Al2O3 R2O & Del Gp newlv Al2O3 R2O & Del Gp newlv Al2O3 R2O & Del Gp newlv Al2O3 R2O Neph \\
\hline 1 & Case 16 - Batch 1 & 45 & Del Gp newlv Al2O3 R2O & Del Gp newlv Al2O3 R2O & Del Gp newlv Al2O3 R2O & Del Gp newlv Al2O3 R2O Neph & Del Gp newlv Al2O3 R2O Neph \\
\hline 1 & Case 16 - Batch 1 & 46 & Del Gp newlv Al2O3 R2O & Del Gp newlv Al2O3 R2O & Del Gp newlv Al2O3 R2O Neph & Del Gp newlv Al2O3 R2O Neph & Del Gp newlv Al2O3 R2O Neph \\
\hline 1 & Case 16 - Batch 1 & 47 & Del Gp newlv Al2O3 R2O & Del Gp newlv Al2O3 R2O Neph & Del Gp newlv Al2O3 R2O Neph & Del Gp newlv Al2O3 R2O Neph & Del Gp newlv Al2O3 R2O Neph \\
\hline 1 & Case 16 - Batch 1 & 48 & Del Gp newlv Al2O3 R2O & Del Gp newlv Al2O3 R2O Neph & Del Gp newlv Al2O3 R2O Neph & Del Gp newlv Al2O3 R2O Neph & Del Gp newlv Al2O3 R2O Neph \\
\hline 1 & Case 16 - Batch 1 & 49 & Del Gp newlv Al2O3 R2O Neph & Del Gp newlv Al2O3 R2O Neph & Del Gp newlv Al2O3 R2O Neph & Del Gp newlv Al2O3 R2O Neph & Del Gp newlv Al2O3 R2O Neph \\
\hline 1 & Case 16 - Batch 1 & 50 & Del Gp newlv Al2O3 R2O Neph & Del Gp newlv Al2O3 R2O Neph & Del Gp newlv Al2O3 R2O Neph & Del Gp newlv Al2O3 R2O Neph & Del Gp newlv Al2O3 R2O Neph \\
\hline 1 & Case 16 - Batch 1 & 51 & Del Gp newlv R2O Neph & Del Gp newlv R2O Neph & Del Gp newlv R2O Neph & Del Gp newlv R2O Neph & Del Gp newlv R2O Neph \\
\hline 1 & Case 16 - Batch 1 & 52 & Del Gp newlv R2O Neph & Del Gp newlv R2O Neph & Del Gp newlv R2O Neph & Del Gp newlv R2O Neph & Del Gp newlv R2O Neph \\
\hline 1 & Case 16 - Batch 1 & 53 & Del Gp newlv R2O Neph & Del Gp newlv R2O Neph & Del Gp newlv R2O Neph & Del Gp newlv R2O Neph & Del Gp newlv R2O Neph \\
\hline 1 & Case 16 - Batch 1 & 54 & Del Gp newlv R2O Neph & Del Gp newlv R2O Neph & Del Gp newlv R2O Neph & Del Gp newlv R2O Neph & Del Gp newlv R2O Neph \\
\hline 1 & Case 16 - Batch 1 & 55 & Del Gp newlv R2O Neph & Del Gp newlv R2O Neph & Del Gp newlv R2O Neph & Del Gp newlv R2O Neph & Del Gp newlv R2O Neph \\
\hline 1 & Case 16 - Batch 1 & 56 & Del Gp newlv R2O Neph & Del Gp newlv R2O Neph & Del Gp newlv R2O Neph & Del Gp newlv R2O Neph & Del Gp newlv R2O Neph \\
\hline 1 & Case 16 - Batch 1 & 57 & Del Gp newlv R2O Neph & Del Gp newlv R2O Neph & Del Gp newlv R2O Neph & Del Gp newlv R2O Neph & Del Gp newlv R2O Neph \\
\hline 1 & Case 16 - Batch 1 & 58 & Del Gp newlv R2O Neph & Del Gp newlv R2O Neph & Del Gp newlv R2O Neph & Del Gp newlv R2O Neph & Del Gp newlv R2O Neph \\
\hline 1 & Case 16 - Batch 1 & 59 & Del Gp newlv R2O Neph & Del Gp newlv R2O Neph & Del Gp newlv R2O Neph & Del Gp newlv R2O Neph & Del Gp newlv R2O Neph \\
\hline 1 & Case 16 - Batch 1 & 60 & Del Gp newlv R2O Neph & Del Gp newlv R2O Neph & Del Gp newlv R2O Neph & Del Gp newlv R2O Neph & Del Gp newlv R2O Neph \\
\hline 1 & Case 16 - Batch 2 & 25 & & & & & \\
\hline 1 & Case 16 - Batch 2 & 26 & & & & & \\
\hline 1 & Case 16 - Batch 2 & 27 & & & & & \\
\hline 1 & Case 16 - Batch 2 & 28 & & & & & \\
\hline 1 & Case 16 - Batch 2 & 29 & & & & & \\
\hline 1 & Case 16 - Batch 2 & 30 & & & & & \\
\hline 1 & Case 16 - Batch 2 & 31 & & & & & \\
\hline 1 & Case 16 - Batch 2 & 32 & & & & & \\
\hline 1 & Case 16 - Batch 2 & 33 & & & & & Neph \\
\hline 1 & Case 16 - Batch 2 & 34 & & & & Neph & Neph \\
\hline 1 & Case 16 - Batch 2 & 35 & & & Neph & Neph & Neph \\
\hline 1 & Case 16 - Batch 2 & 36 & & Neph & Neph & Neph & Neph \\
\hline 1 & Case 16 - Batch 2 & 37 & & Neph & Neph & Neph & Neph \\
\hline 1 & Case 16 - Batch 2 & 38 & Neph & Neph & Neph & Neph & Neph \\
\hline 1 & Case 16 - Batch 2 & 39 & Neph & Neph & Neph & Neph & Neph \\
\hline 1 & Case 16 - Batch 2 & 40 & Neph & Neph & Neph & Neph & Neph \\
\hline 1 & Case 16 - Batch 2 & 41 & Neph & Neph & Neph & Neph & Neph \\
\hline 1 & Case 16 - Batch 2 & 42 & Neph & Neph & Neph & Neph & Neph \\
\hline 1 & Case 16 - Batch 2 & 43 & Neph & Neph & Neph & Neph & Neph \\
\hline 1 & Case 16 - Batch 2 & 44 & Neph & Neph & Neph & Neph & Neph \\
\hline 1 & Case 16 - Batch 2 & 45 & Neph & Neph & Neph & Neph & Neph \\
\hline 1 & Case 16 - Batch 2 & 46 & Neph & Neph & Neph & Neph & Neph \\
\hline 1 & Case 16 - Batch 2 & 47 & Neph & Neph & Neph & Neph & Neph \\
\hline 1 & Case 16 - Batch 2 & 48 & Neph & Neph & Neph & Neph & Neph \\
\hline
\end{tabular}


Exhibit A1. Results for MAR Assessments of the Nominal Stage (part 4)

Constraint not met: neph (nepheline), TL (liquidus temperature), newlv (low viscosity - new model), newhv (high viscosity - new model), and Del GP - durability

\begin{tabular}{|c|c|c|c|c|c|c|c|}
\hline & & $\mathrm{Na} 2 \mathrm{O}$ & 0.11 & 0.12 & 0.13 & 0.14 & 0.15 \\
\hline Set & Type & Sludge Loading (\%) & 417 & 320 & 431 & $\mathrm{Y} 1-1$ & $\mathrm{X1-1}$ \\
\hline 1 & Case 16 - Batch 2 & 49 & Neph & Neph & Neph & Neph & Neph \\
\hline 1 & Case 16 - Batch 2 & 50 & Neph & Neph & Neph & Neph & Neph \\
\hline 1 & Case 16 - Batch 2 & 51 & Neph & Neph & Neph & Neph & Neph \\
\hline 1 & Case 16- Batch 2 & 52 & Neph & Neph & Neph & Neph & Neph \\
\hline 1 & Case 16 - Batch 2 & 53 & Neph & Neph & Neph & Neph & Neph \\
\hline 1 & Case 16 - Batch 2 & 54 & Neph & Neph & Neph & Neph & Neph \\
\hline 1 & Case 16 - Batch 2 & 55 & Neph & Neph & Neph & Neph & Neph \\
\hline 1 & Case 16 - Batch 2 & 56 & TL Neph & Neph & Neph & Neph & Neph \\
\hline 1 & Case 16 - Batch 2 & 57 & TL Neph & TL Neph & TL Neph & Neph & Neph \\
\hline 1 & Case 16 - Batch 2 & 58 & TL Neph & TL Neph & TL Neph & TL Neph & newlv Neph \\
\hline 1 & Case 16 - Batch 2 & 59 & TL Neph & TL Neph & TL Neph & TL Neph & TL newlv Neph \\
\hline 1 & Case 16 - Batch 2 & 60 & TL Neph & TL Neph & TL Neph & TL Neph & TL newlv Neph \\
\hline 1 & Case 16 - Blend 1 & 25 & $\mathrm{R} 2 \mathrm{O}$ & $\mathrm{R} 2 \mathrm{O}$ & Del Gp R2O & Del Gp R2O & Del Gp newlv R2O \\
\hline 1 & Case 16 - Blend 1 & 26 & $\mathrm{R} 2 \mathrm{O}$ & $\mathrm{R} 2 \mathrm{O}$ & Del Gp R2O & Del Gp R2O & Del Gp newlv R2O \\
\hline 1 & Case 16 - Blend 1 & 27 & $\mathrm{R} 2 \mathrm{O}$ & $\mathrm{R} 2 \mathrm{O}$ & Del Gp R2O & Del Gp newlv R2O & Del Gp newlv R2O \\
\hline 1 & Case 16 - Blend 1 & 28 & $\mathrm{R} 2 \mathrm{O}$ & $\mathrm{R} 2 \mathrm{O}$ & Del Gp R2O & Del Gp newlv R2O & Del Gp newlv R2O \\
\hline 1 & Case 16 - Blend 1 & 29 & $\mathrm{R} 2 \mathrm{O}$ & $\mathrm{R} 2 \mathrm{O}$ & Del Gp R2O & Del Gp newlv R2O & $\begin{array}{l}\text { Del Gp newlv R2O } \\
\end{array}$ \\
\hline 1 & Case 16 - Blend 1 & 30 & & & Del Gp & Del Gp newlv & Del Gp newlv \\
\hline 1 & Case 16 - Blend 1 & 31 & & & Del Gp newlv & Del Gp newlv & Del Gp newlv \\
\hline 1 & Case 16 - Blend 1 & 32 & & & Del Gp newlv & Del Gp newlv & Del Gp newlv \\
\hline 1 & Case 16 - Blend 1 & 33 & & & Del Gp newlv & Del Gp newlv & Del Gp newlv \\
\hline 1 & Case 16 - Blend 1 & 34 & & newlv & Del Gp newlv & Del Gp newlv & Del Gp newlv \\
\hline 1 & Case 16 - Blend 1 & 35 & & newlv & newlv & Del Gp newlv & Del Gp newlv \\
\hline 1 & Case 16 - Blend 1 & 36 & & newlv & newlv & Del Gp newlv & Del Gp newlv \\
\hline 1 & Case 16 - Blend 1 & 37 & newlv & newlv & newlv & Del Gp newlv & Del Gp newlv \\
\hline 1 & Case 16 - Blend 1 & 38 & newlv & newlv & newlv & Del Gp newlv & Del Gp newlv \\
\hline 1 & Case 16 - Blend 1 & 39 & newlv & newlv & newlv & Del Gp newlv & Del Gp newlv \\
\hline 1 & $\begin{array}{l}\text { Case } 16 \text { - Blend } 1 \\
\end{array}$ & 40 & newlv & newlv & newlv & Del Gp newlv & Del Gp newlv \\
\hline 1 & Case 16 - Blend 1 & 41 & newlv & newlv & newlv & Del Gp newlv & Del Gp newlv \\
\hline 1 & Case 16 - Blend 1 & 42 & newlv & newlv & newlv & Del Gp newlv & Del Gp newlv \\
\hline 1 & Case 16 - Blend 1 & 43 & newlv & newlv & newlv & Del Gp newlv & Del Gp newlv \\
\hline 1 & Case 16 - Blend 1 & 44 & newlv & newlv & newlv & Del Gp newlv & Del Gp newlv \\
\hline 1 & Case 16 - Blend 1 & 45 & TL newlv & newlv & newlv & Del Gp newlv & Del Gp newlv \\
\hline 1 & Case 16 - Blend 1 & 46 & TL newlv & TL newlv & newlv & Del Gp newlv & Del Gp newlv \\
\hline 1 & Case 16 - Blend 1 & 47 & TL newlv & TL newlv & TL newlv & Del Gp newlv & Del Gp newlv Neph \\
\hline 1 & Case 16 - Blend 1 & 48 & TL newlv & TL newlv & TL newlv & Del Gp TL newlv Neph & Del Gp newlv Neph \\
\hline 1 & Case 16 - Blend 1 & 49 & TL newly & TL newlv & TL newlv & Del Gp TL newlv Neph & Del Gp TL newlv Neph \\
\hline 1 & Case 16 - Blend 1 & 50 & TL newly & TL newlv & TL newlv Neph & Del Gp TL newlv Neph & Del Gp TL newlv Neph \\
\hline 1 & Case 16 - Blend 1 & 51 & TL newlv & TL newlv Neph & TL newlv Neph & Del Gp TL newlv Neph & Del Gp TL newlv Neph \\
\hline 1 & Case 16 - Blend 1 & 52 & TL newlv Neph & TL newlv Neph & TL newlv Neph & Del Gp TL newlv Neph & Del Gp TL newlv Neph \\
\hline 1 & Case 16 - Blend 1 & 53 & TL newlv Neph & TL newlv Neph & TL newlv Neph & Del Gp TL newlv Neph & Del Gp TL newlv Neph \\
\hline 1 & Case 16 - Blend 1 & 54 & TL newlv Neph & TL newlv Neph & TL newlv Neph & Del Gp TL newlv Neph & Del Gp TL newlv Neph \\
\hline 1 & Case 16 - Blend 1 & 55 & TL newlv Neph & TL newlv Neph & TL newlv Neph & Del Gp TL newlv Neph & Del Gp TL newlv Neph \\
\hline 1 & Case 16 - Blend 1 & 56 & TL newlv Neph & TL newlv Neph & TL newlv Neph & Del Gp TL newlv Neph & Del Gp TL newlv Neph \\
\hline 1 & Case 16 - Blend 1 & 57 & TL newlv Neph & TL newlv Neph & TL newlv Neph & Del Gp TL newlv Neph & Del Gp TL newlv Neph \\
\hline 1 & Case 16 - Blend 1 & 58 & TL newlv Neph & TL newlv Neph & TL newlv Neph & Del Gp TL newlv Neph & Del Gp TL newlv Neph \\
\hline 1 & Case 16 - Blend 1 & 59 & TL newlv Neph & TL newlv Neph & TL newlv Neph & Del Gp TL newlv Neph & Del Gp TL newlv Neph \\
\hline 1 & Case 16 - Blend 1 & 60 & TL newlv Neph & TL newlv Neph & TL newlv Neph & TL newlv Neph & Del Gp TL newlv Neph \\
\hline 1 & Case 16 - Blend 2 & 25 & & & & & Del Gp \\
\hline 1 & Case 16 - Blend 2 & 26 & & & & & Del Gp \\
\hline 1 & Case 16 - Blend 2 & 27 & & & & & Del Gp \\
\hline
\end{tabular}


Exhibit A1. Results for MAR Assessments of the Nominal Stage (part 4)

Constraint not met: neph (nepheline), TL (liquidus temperature), newlv (low viscosity - new model), newhv (high viscosity - new model), and Del GP - durability

\begin{tabular}{|c|c|c|c|c|c|c|c|}
\hline & & $\mathrm{Na} 2 \mathrm{O}$ & 0.11 & 0.12 & 0.13 & 0.14 & 0.15 \\
\hline Set & $\begin{array}{l}\text { Type } \\
\end{array}$ & $\begin{array}{l}\text { Sludge Loading (\%) } \\
\end{array}$ & 417 & 320 & 431 & $\mathrm{Y} 1-1$ & $\mathrm{X} 1-1$ \\
\hline 1 & Case $16-$ Blend 2 & 28 & & & & & Del Gp \\
\hline 1 & Case 16 - Blend 2 & 29 & & & & & Del Gp \\
\hline 1 & Case 16 - Blend 2 & 30 & & & & & Del Gp \\
\hline 1 & Case 16 - Blend 2 & 31 & & & & & \\
\hline 1 & Case 16 - Blend 2 & 32 & & & & & \\
\hline 1 & Case 16 - Blend 2 & 33 & & & & & \\
\hline 1 & Case 16 - Blend 2 & 34 & & & & & \\
\hline 1 & Case 16 - Blend 2 & 35 & & & & & Neph \\
\hline 1 & Case 16 - Blend 2 & 36 & & & & & Neph \\
\hline 1 & Case 16 - Blend 2 & 37 & & & & Neph & Neph \\
\hline 1 & Case 16 - Blend 2 & 38 & & & Neph & Neph & Neph \\
\hline 1 & Case 16 - Blend 2 & 39 & & Neph & Neph & Neph & Neph \\
\hline 1 & Case 16 - Blend 2 & 40 & Neph & Neph & Neph & Neph & Neph \\
\hline 1 & Case 16 - Blend 2 & 41 & Neph & Neph & Neph & Neph & newlv Neph \\
\hline 1 & Case 16 - Blend 2 & 42 & Neph & Neph & Neph & Neph & newlv Neph \\
\hline 1 & Case 16 - Blend 2 & 43 & Neph & Neph & Neph & Neph & newlv Neph \\
\hline 1 & Case 16 - Blend 2 & 44 & Neph & Neph & Neph & Neph & newlv Neph \\
\hline 1 & Case 16 - Blend 2 & 45 & Neph & Neph & Neph & Neph & newlv Neph \\
\hline 1 & Case 16 - Blend 2 & 46 & Neph & Neph & Neph & Neph & newlv Neph \\
\hline 1 & Case 16 - Blend 2 & 47 & Neph & Neph & Neph & newlv Neph & newlv Neph \\
\hline 1 & Case 16 - Blend 2 & 48 & Neph & Neph & Neph & newlv Neph & newlv Neph \\
\hline 1 & Case 16 - Blend 2 & 49 & Neph & Neph & Neph & newlv Neph & newlv Neph \\
\hline 1 & Case 16 - Blend 2 & 50 & Neph & Neph & Neph & newlv Neph & newlv Neph \\
\hline 1 & Case 16 - Blend 2 & 51 & TL Neph & Neph & newlv Neph & newlv Neph & newlv Neph \\
\hline 1 & Case 16 - Blend 2 & 52 & TL Neph & TL Neph & newlv Neph & newlv Neph & newlv Neph \\
\hline 1 & Case 16 - Blend 2 & 53 & TL Neph & TL Neph & TL newlv Neph & newlv Neph & newlv Neph \\
\hline 1 & Case 16 - Blend 2 & 54 & TL Neph & TL Neph & TL newlv Neph & TL newlv Neph & newlv Neph \\
\hline 1 & Case 16 - Blend 2 & 55 & TL Neph & TL newlv Neph & TL newlv Neph & TL newlv Neph & TL newlv Neph \\
\hline 1 & Case 16 - Blend 2 & 56 & TL Neph & TL newlv Neph & TL newlv Neph & TL newlv Neph & TL newlv Neph \\
\hline 1 & Case 16 - Blend 2 & 57 & TL Neph & TL newlv Neph & TL newlv Neph & TL newlv Neph & TL newlv Neph \\
\hline 1 & Case 16 - Blend 2 & 58 & TL newlv Neph & TL newlv Neph & TL newlv Neph & TL newlv Neph & TL newlv Neph \\
\hline 1 & Case 16 - Blend 2 & 59 & TL newlv Neph & TL newlv Neph & TL newlv Neph & TL newlv Neph & TL newlv Neph \\
\hline 1 & Case 16 - Blend 2 & 60 & TL newlv Neph & TL newlv Neph & TL newlv Neph & TL newlv Neph & TL newlv Neph \\
\hline 1 & Case 7 Batch 1 & 25 & & & & Del Gp & Del Gp \\
\hline 1 & Case 7 Batch 1 & 26 & & & & Del Gp & Del Gp \\
\hline 1 & Case 7 Batch 1 & 27 & & & & Del Gp & Del Gp \\
\hline 1 & Case 7 Batch 1 & 28 & & & & Del Gp & Del Gp \\
\hline 1 & Case 7 Batch 1 & 29 & & & & Del Gp & Del Gp \\
\hline 1 & Case 7 Batch 1 & 30 & & & & & Del Gp \\
\hline 1 & Case 7 Batch 1 & 31 & & & & & Del Gp \\
\hline 1 & Case 7 Batch 1 & 32 & & & & & Del Gp \\
\hline 1 & Case 7 Batch 1 & 33 & & & & & Del Gp \\
\hline 1 & Case 7 Batch 1 & 34 & & & & & Del Gp Neph \\
\hline 1 & Case 7 Batch 1 & 35 & & & & & Del Gp Neph \\
\hline 1 & Case 7 Batch 1 & 36 & & & & Neph & Del Gp Neph \\
\hline 1 & Case 7 Batch 1 & 37 & & & Neph & Neph & Del Gp Neph \\
\hline 1 & Case 7 Batch 1 & 38 & & Neph & Neph & Neph & Del Gp newlv Neph \\
\hline 1 & Case 7 Batch 1 & 39 & Neph & Neph & Neph & Neph & Del Gp newlv Neph \\
\hline 1 & Case 7 Batch 1 & 40 & Neph & Neph & Neph & Neph & Del Gp newlv Neph \\
\hline 1 & Case 7 Batch 1 & 41 & Neph & Neph & Neph & Neph & Del Gp newlv Neph \\
\hline 1 & Case 7 Batch 1 & 42 & Neph & Neph & Neph & Neph & newlv Neph \\
\hline
\end{tabular}


Exhibit A1. Results for MAR Assessments of the Nominal Stage (part 4)

Constraint not met: neph (nepheline), TL (liquidus temperature), newlv (low viscosity - new model), newhv (high viscosity - new model), and Del GP - durability

\begin{tabular}{|c|c|c|c|c|c|c|c|}
\hline & & $\mathrm{Na} 2 \mathrm{O}$ & 0.11 & 0.12 & 0.13 & 0.14 & 0.15 \\
\hline Set & Type & Sludge Loading (\%) & 417 & 320 & 431 & $\mathrm{Y} 1-1$ & $\mathrm{X} 1-1$ \\
\hline 1 & Case 7 Batch 1 & 43 & Neph & Neph & Neph & newlv Neph & newlv Neph \\
\hline 1 & Case 7 Batch 1 & 44 & Neph & Neph & Neph & newlv Neph & newlv Neph \\
\hline 1 & Case 7 Batch 1 & 45 & Neph & Neph & Neph & $\begin{array}{l}\text { newlv Neph } \\
\end{array}$ & newlv Neph \\
\hline 1 & Case 7 Batch 1 & 46 & Neph & Neph & Neph & newlv Neph & newlv Neph \\
\hline 1 & Case 7 Batch 1 & 47 & Neph & Neph & Neph & newlv Neph & newlv Neph \\
\hline 1 & Case 7 Batch 1 & 48 & Neph & Neph & newlv Neph & newlv Neph & newlv Neph \\
\hline 1 & Case 7 Batch 1 & 49 & Neph & Neph & newlv Neph & newlv Neph & newlv Neph \\
\hline 1 & Case 7 Batch 1 & 50 & Neph & Neph & newlv Neph & newlv Neph & newlv Neph \\
\hline 1 & Case 7 Batch 1 & 51 & Neph & Neph & newlv Neph & newlv Neph & newlv Neph \\
\hline 1 & Case 7 Batch 1 & 52 & Neph & newlv Neph & newlv Neph & newlv Neph & newlv Neph \\
\hline 1 & Case 7 Batch 1 & 53 & Neph & newlv Neph & newlv Neph & newlv Neph & newlv Neph \\
\hline 1 & Case 7 Batch 1 & 54 & TL Neph & newlv Neph & newlv Neph & newlv Neph & newlv Neph \\
\hline 1 & Case 7 Batch 1 & 55 & TL newlv Neph & TL newlv Neph & newlv Neph & newlv Neph & newlv Neph \\
\hline 1 & Case 7 Batch 1 & 56 & TL newlv Neph & TL newlv Neph & TL newlv Neph & TL newlv Neph & newlv Neph \\
\hline 1 & Case 7 Batch 1 & 57 & TL newlv Neph & TL newlv Neph & TL newlv Neph & TL newlv Neph & TL newlv Nepl \\
\hline 1 & Case 7 Batch 1 & 58 & TL newlv Neph & TL newlv Neph & TL newlv Neph & TL newlv Neph & TL newlv Nepl \\
\hline 1 & Case 7 Batch 1 & 59 & TL newlv Neph & TL newlv Neph & TL newlv Neph & TL newlv Neph & TL newlv Nepl \\
\hline 1 & Case 7 Batch 1 & 60 & TL newlv Neph & TL newlv Neph & TL newlv Neph & TL newlv Neph & TL newlv Nepl \\
\hline 1 & Case 7 Batch 2 & 25 & & & & & \\
\hline 1 & Case 7 Batch 2 & 26 & & & & & \\
\hline 1 & Case 7 Batch 2 & 27 & & & & & \\
\hline 1 & Case 7 Batch 2 & 28 & & & & & \\
\hline 1 & Case 7 Batch 2 & 29 & & & & & \\
\hline 1 & Case 7 Batch 2 & 30 & & & & & \\
\hline 1 & Case 7 Batch 2 & 31 & & & & & \\
\hline 1 & Case 7 Batch 2 & 32 & & & & & \\
\hline 1 & Case 7 Batch 2 & 33 & & & & & \\
\hline 1 & Case 7 Batch 2 & 34 & & & & & \\
\hline 1 & Case 7 Batch 2 & 35 & & & & & Neph \\
\hline 1 & Case 7 Batch 2 & 36 & & & & & Neph \\
\hline 1 & Case 7 Batch 2 & 37 & & & & Neph & Neph \\
\hline 1 & Case 7 Batch 2 & 38 & & & Neph & Neph & Neph \\
\hline 1 & Case 7 Batch 2 & 39 & & Neph & Neph & Neph & Neph \\
\hline 1 & Case 7 Batch 2 & 40 & Neph & Neph & Neph & Neph & Neph \\
\hline 1 & Case 7 Batch 2 & 41 & Neph & Neph & Neph & Neph & Neph \\
\hline 1 & Case 7 Batch 2 & 42 & Neph & Neph & Neph & Neph & Neph \\
\hline 1 & Case 7 Batch 2 & 43 & Neph & Neph & Neph & Neph & Neph \\
\hline 1 & Case 7 Batch 2 & 44 & Neph & Neph & Neph & Neph & Neph \\
\hline 1 & Case 7 Batch 2 & 45 & Neph & Neph & Neph & Neph & Neph \\
\hline 1 & Case 7 Batch 2 & 46 & Neph & Neph & Neph & Neph & Neph \\
\hline 1 & Case 7 Batch 2 & 47 & TL Neph & TL Neph & Neph & Neph & Neph \\
\hline 1 & Case 7 Batch 2 & 48 & TL Neph & TL Neph & TL Neph & Neph & Neph \\
\hline 1 & Case 7 Batch 2 & 49 & TL Neph & TL Neph & TL Neph & TL Neph & Neph \\
\hline 1 & Case 7 Batch 2 & 50 & TL Neph & TL Neph & TL Neph & TL Neph & TL Neph \\
\hline 1 & Case 7 Batch 2 & 51 & TL Neph & TL Neph & TL Neph & TL Neph & TL Neph \\
\hline 1 & Case 7 Batch 2 & 52 & TL Neph & TL Neph & TL Neph & TL Neph & TL Neph \\
\hline 1 & Case 7 Batch 2 & 53 & TL Neph & TL Neph & TL Neph & TL Neph & TL Neph \\
\hline 1 & Case 7 Batch 2 & 54 & TL Neph & TL Neph & TL Neph & TL Neph & TL Neph \\
\hline 1 & Case 7 Batch 2 & 55 & TL Neph & TL Neph & TL Neph & TL Neph & TL Neph \\
\hline 1 & Case 7 Batch 2 & 56 & TL Neph & TL Neph & TL Neph & TL Neph & TL newlv Nepl \\
\hline 1 & Case 7 Batch 2 & 57 & TL Neph & TL Neph & TL Neph & TL Neph & TL newlv Nepl \\
\hline
\end{tabular}


Exhibit A1. Results for MAR Assessments of the Nominal Stage (part 4)

Constraint not met: neph (nepheline), TL (liquidus temperature), newlv (low viscosity - new model), newhv (high viscosity - new model), and Del GP - durability

\begin{tabular}{|c|c|c|c|c|c|c|c|}
\hline & & $\mathrm{Na} 2 \mathrm{O}$ & 0.11 & 0.12 & 0.13 & 0.14 & 0.15 \\
\hline Set & Type & Sludge Loading (\%) & 417 & 320 & 431 & $\mathrm{Y} 1-1$ & $\mathrm{X} 1-1$ \\
\hline 1 & Case 7 Batch 2 & 58 & TL Neph & TL Neph & TL Neph & TL Neph & TL newlv Neph \\
\hline 1 & Case 7 Batch 2 & 59 & TL Neph & TL Neph & TL Neph & TL Neph & TL newlv Neph \\
\hline 1 & Case 7 Batch 2 & 60 & TL Neph & TL Neph & TL Neph & TL Neph & TL newlv Neph \\
\hline 1 & Case 7 Blend 1 & 25 & & & Del Gp & Del Gp & Del Gp newlv \\
\hline 1 & Case 7 Blend 1 & 26 & & & Del Gp & Del Gp & Del Gp newlv \\
\hline 1 & Case 7 Blend 1 & 27 & & & Del Gp & Del Gp & Del Gp newlv \\
\hline 1 & Case 7 Blend 1 & 28 & & & Del Gp & Del Gp newlv & Del Gp newlv \\
\hline 1 & Case 7 Blend 1 & 29 & & & Del Gp & Del Gp newlv & Del Gp newlv \\
\hline 1 & Case 7 Blend 1 & 30 & & & Del Gp & Del Gp newlv & Del Gp newlv \\
\hline 1 & Case 7 Blend 1 & 31 & & & Del Gp & Del Gp newlv & Del Gp newlv \\
\hline 1 & Case 7 Blend 1 & 32 & & & Del Gp newlv & Del Gp newlv & Del Gp newlv \\
\hline 1 & Case 7 Blend 1 & 33 & & & Del Gp newlv & Del Gp newlv & Del Gp newlv \\
\hline 1 & Case 7 Blend 1 & 34 & & & Del Gp newlv & Del Gp newlv & Del Gp newlv \\
\hline 1 & Case 7 Blend 1 & 35 & & newlv & Del Gp newlv & Del Gp newlv & Del Gp newlv \\
\hline 1 & Case 7 Blend 1 & 36 & & newlv & Del Gp newlv & Del Gp newlv & Del Gp newlv \\
\hline 1 & $\begin{array}{l}\text { Case } 7 \text { Blend } 1 \\
\end{array}$ & 37 & & newlv & Del Gp newlv & Del Gp newlv & Del Gp newlv \\
\hline 1 & Case 7 Blend 1 & 38 & newlv & newlv & Del Gp newlv & Del Gp newlv & Del Gp newlv \\
\hline 1 & Case 7 Blend 1 & 39 & newlv & newlv & Del Gp newlv & Del Gp newlv & Del Gp newlv \\
\hline 1 & Case 7 Blend 1 & 40 & newlv & newlv & Del Gp newlv & Del Gp newlv & Del Gp newlv \\
\hline 1 & Case 7 Blend 1 & 41 & newlv & newlv & Del Gp newlv & Del Gp newlv & Del Gp newlv Neph \\
\hline 1 & Case 7 Blend 1 & 42 & newlv & newlv & Del Gp newlv & Del Gp newlv & Del Gp newlv Neph \\
\hline 1 & Case 7 Blend 1 & 43 & newlv & newlv & Del Gp newlv & Del Gp newlv Neph & Del Gp newlv Neph \\
\hline 1 & $\begin{array}{l}\text { Case } 7 \text { Blend } 1 \\
\end{array}$ & 44 & newlv & newlv & Del Gp newlv Neph & Del Gp newlv Neph & Del Gp newlv Neph \\
\hline 1 & Case 7 Blend 1 & 45 & newlv & newlv Neph & Del Gp newlv Neph & Del Gp newlv Neph & Del Gp newlv Neph \\
\hline 1 & Case 7 Blend 1 & 46 & newlv & newlv Neph & Del Gp newlv Neph & Del Gp newlv Neph & Del Gp newlv Neph \\
\hline 1 & $\begin{array}{l}\text { Case } 7 \text { Blend } 1 \\
\end{array}$ & 47 & newlv Neph & newlv Neph & Del Gp newlv Neph & Del Gp newlv Neph & Del Gp newlv Neph \\
\hline 1 & Case 7 Blend 1 & 48 & newlv Neph & newlv Neph & Del Gp newlv Neph & Del Gp newlv Neph & Del Gp newlv Neph \\
\hline 1 & $\begin{array}{l}\text { Case } 7 \text { Blend } 1 \\
\end{array}$ & 49 & newlv Neph & newlv Neph & Del Gp newlv Neph & Del Gp newlv Neph & Del Gp newlv Neph \\
\hline 1 & Case 7 Blend 1 & 50 & newlv Neph & newlv Neph & Del Gp newlv Neph & Del Gp newlv Neph & Del Gp newlv Neph \\
\hline 1 & Case 7 Blend 1 & 51 & TL newlv Neph & newlv Neph & Del Gp newlv Neph & Del Gp newlv Neph & Del Gp newlv Neph \\
\hline 1 & $\begin{array}{l}\text { Case } 7 \text { Blend } 1 \\
\end{array}$ & 52 & TL newly Neph & TL newlv Neph & Del Gp newlv Neph & Del Gp newlv Neph & Del Gp newlv Neph \\
\hline 1 & Case 7 Blend 1 & 53 & TL newlv Neph & TL newlv Neph & Del Gp TL newlv Neph & Del Gp TL newlv Neph & Del Gp newlv Neph \\
\hline 1 & Case 7 Blend 1 & 54 & TL newlv Neph & TL newlv Neph & Del Gp TL newlv Neph & Del Gp TL newlv Neph & Del Gp TL newlv Neph \\
\hline 1 & Case 7 Blend 1 & 55 & TL newlv Neph & TL newlv Neph & Del Gp TL newlv Neph & Del Gp TL newlv Neph & Del Gp TL newlv Neph \\
\hline 1 & Case 7 Blend 1 & 56 & TL newlv Neph & TL newlv Neph & Del Gp TL newlv Neph & Del Gp TL newlv Neph & Del Gp TL newlv Neph \\
\hline 1 & Case 7 Blend 1 & 57 & TL newlv Neph & TL newlv Neph & Del Gp TL newlv Neph & Del Gp TL newlv Neph & Del Gp TL newlv Neph \\
\hline 1 & Case 7 Blend 1 & 58 & TL newlv Neph & TL newlv Neph & Del Gp TL newlv Neph & Del Gp TL newlv Neph & Del Gp TL newlv Neph \\
\hline 1 & $\begin{array}{l}\text { Case } 7 \text { Blend } 1 \\
\end{array}$ & 59 & TL newly Neph & TL newlv Neph & Del Gp TL newlv Neph & Del Gp TL newlv Neph & Del Gp TL newlv Neph \\
\hline 1 & Case 7 Blend 1 & 60 & TL newlv Neph & TL newlv Neph & Del Gp TL newlv Neph & Del Gp TL newlv Neph & Del Gp TL newlv Neph \\
\hline 1 & Case 7 Blend 2 & 25 & & & & & Del Gp \\
\hline 1 & Case 7 Blend 2 & 26 & & & & & Del Gp \\
\hline 1 & Case 7 Blend 2 & 27 & & & & & Del Gp \\
\hline 1 & Case 7 Blend 2 & 28 & & & & & \\
\hline 1 & Case 7 Blend 2 & 29 & & & & & \\
\hline 1 & Case 7 Blend 2 & 30 & & & & & \\
\hline 1 & Case 7 Blend 2 & 31 & & & & & \\
\hline 1 & Case 7 Blend 2 & 32 & & & & & \\
\hline 1 & Case 7 Blend 2 & 33 & & & & & \\
\hline 1 & Case 7 Blend 2 & 34 & & & & & \\
\hline 1 & Case 7 Blend 2 & 35 & & & & & \\
\hline 1 & Case 7 Blend 2 & 36 & & & & & Neph \\
\hline
\end{tabular}


Exhibit A1. Results for MAR Assessments of the Nominal Stage (part 4)

Constraint not met: neph (nepheline), TL (liquidus temperature), newlv (low viscosity - new model), newhv (high viscosity - new model), and Del GP - durability

\begin{tabular}{|c|c|c|c|c|c|c|c|}
\hline & & $\mathrm{Na} 2 \mathrm{O}$ & 0.11 & 0.12 & 0.13 & 0.14 & 0.15 \\
\hline Set & Type & Sludge Loading (\%) & 417 & 320 & 431 & $\mathrm{Y} 1-1$ & $\mathrm{X} 1-1$ \\
\hline 1 & Case 7 Blend 2 & 37 & & & & & Neph \\
\hline 1 & Case 7 Blend 2 & 38 & & & & Neph & Neph \\
\hline 1 & Case 7 Blend 2 & 39 & & & Neph & Neph & Neph \\
\hline 1 & Case 7 Blend 2 & 40 & & Neph & Neph & Neph & Neph \\
\hline 1 & Case 7 Blend 2 & 41 & Neph & Neph & Neph & Neph & Neph \\
\hline 1 & Case 7 Blend 2 & 42 & Neph & Neph & Neph & Neph & Neph \\
\hline 1 & Case 7 Blend 2 & 43 & Neph & Neph & Neph & Neph & newlv Neph \\
\hline 1 & Case 7 Blend 2 & 44 & Neph & Neph & Neph & Neph & newlv Neph \\
\hline 1 & Case 7 Blend 2 & 45 & Neph & Neph & Neph & Neph & newlv Neph \\
\hline 1 & Case 7 Blend 2 & 46 & Neph & Neph & Neph & Neph & newlv Neph \\
\hline 1 & Case 7 Blend 2 & 47 & TL Neph & Neph & Neph & Neph & newlv Neph \\
\hline 1 & Case 7 Blend 2 & 48 & TL Neph & TL Neph & Neph & newlv Neph & newlv Neph \\
\hline 1 & $\begin{array}{l}\text { Case } 7 \text { Blend } 2 \\
\end{array}$ & 49 & TL Neph & TL Neph & TL Neph & newlv Neph & newlv Neph \\
\hline 1 & Case 7 Blend 2 & 50 & TL Neph & TL Neph & TL Neph & TL newlv Neph & newlv Neph \\
\hline 1 & Case 7 Blend 2 & 51 & TL Neph & TL Neph & TL Neph & TL newlv Neph & TL newlv Neph \\
\hline 1 & Case 7 Blend 2 & 52 & TL Neph & TL Neph & TL Neph & TL newlv Neph & TL newlv Neph \\
\hline 1 & Case 7 Blend 2 & 53 & TL Neph & TL Neph & TL newlv Neph & TL newlv Neph & TL newlv Neph \\
\hline 1 & Case 7 Blend 2 & 54 & TL Neph & TL Neph & TL newlv Neph & TL newlv Neph & TL newlv Neph \\
\hline 1 & Case 7 Blend 2 & 55 & TL Neph & TL Neph & TL newlv Neph & TL newlv Neph & TL newlv Neph \\
\hline 1 & Case 7 Blend 2 & 56 & TL Neph & TL Neph & TL newlv Neph & TL newlv Neph & TL newlv Neph \\
\hline 1 & Case 7 Blend 2 & 57 & TL Neph & TL newlv Neph & TL newlv Neph & TL newlv Neph & TL newlv Neph \\
\hline 1 & Case 7 Blend 2 & 58 & TL Neph & TL newlv Neph & TL newlv Neph & TL newlv Neph & TL newlv Neph \\
\hline 1 & Case 7 Blend 2 & 59 & TL Neph & TL newlv Neph & TL newlv Neph & TL newlv Neph & TL newlv Neph \\
\hline 1 & Case 7 Blend 2 & 60 & TL newlv Neph & TL newlv Neph & TL newlv Neph & TL newlv Neph & TL newlv Neph \\
\hline 1 & Case 8 - Blend 1 & 25 & & & Del Gp & Del Gp & Del Gp \\
\hline 1 & Case 8 - Blend 1 & 26 & & & Del Gp & Del Gp & Del Gp \\
\hline 1 & Case 8 - Blend 1 & 27 & & & Del Gp & Del Gp & Del Gp newlv \\
\hline 1 & Case 8 - Blend 1 & 28 & & & Del Gp & Del Gp & Del Gp newlv \\
\hline 1 & Case 8 - Blend 1 & 29 & & & Del Gp & Del Gp & Del Gp newlv \\
\hline 1 & Case 8 - Blend 1 & 30 & & & Del Gp & Del Gp & Del Gp newlv \\
\hline 1 & Case 8 - Blend 1 & 31 & & & Del Gp & Del Gp & Del Gp newlv \\
\hline 1 & Case 8 - Blend 1 & 32 & & & & Del Gp newlv & Del Gp newlv \\
\hline 1 & Case 8 - Blend 1 & 33 & & & & Del Gp newlv & Del Gp newlv \\
\hline 1 & Case 8 - Blend 1 & 34 & & & & Del Gp newlv & Del Gp newlv \\
\hline 1 & Case 8 - Blend 1 & 35 & & & & Del Gp newlv & Del Gp newlv \\
\hline 1 & Case 8 - Blend 1 & 36 & & & newlv & Del Gp newlv & Del Gp newlv \\
\hline 1 & Case 8 - Blend 1 & 37 & & & newlv & Del Gp newlv & Del Gp newlv \\
\hline 1 & Case 8 - Blend 1 & 38 & & & newlv & Del Gp newlv & Del Gp newlv \\
\hline 1 & Case 8 - Blend 1 & 39 & & & newlv & Del Gp newlv & Del Gp newlv Nepl \\
\hline 1 & Case 8 - Blend 1 & 40 & & newlv & newlv & Del Gp newlv Neph & Del Gp newlv Nepl \\
\hline 1 & Case 8 - Blend 1 & 41 & & newlv & newlv Neph & Del Gp newlv Neph & Del Gp newlv Nepl \\
\hline 1 & Case 8 - Blend 1 & 42 & & newlv & newlv Neph & Del Gp newlv Neph & Del Gp newlv Nepl \\
\hline 1 & Case 8 - Blend 1 & 43 & newlv & newlv Neph & newlv Neph & Del Gp newlv Neph & Del Gp newlv Nepl \\
\hline 1 & Case 8 - Blend 1 & 44 & newlv Neph & newlv Neph & newlv Neph & Del Gp newlv Neph & Del Gp newlv Nepl \\
\hline 1 & Case 8 - Blend 1 & 45 & newlv Neph & newlv Neph & newlv Neph & Del Gp newlv Neph & Del Gp newlv Nepl \\
\hline 1 & Case 8 - Blend 1 & 46 & newlv Neph & newlv Neph & newlv Neph & Del Gp newlv Neph & Del Gp newlv Nepl \\
\hline 1 & Case 8 - Blend 1 & 47 & newlv Neph & newlv Neph & newlv Neph & Del Gp newlv Neph & Del Gp newlv Nepl \\
\hline 1 & Case 8 - Blend 1 & 48 & newlv Neph & newlv Neph & newlv Neph & Del Gp newlv Neph & Del Gp newlv Nepl \\
\hline 1 & Case 8 - Blend 1 & 49 & newlv Neph & newlv Neph & newlv Neph & Del Gp newlv Neph & Del Gp newlv Nepl \\
\hline 1 & Case 8 - Blend 1 & 50 & TL newlv Neph & TL newlv Neph & newlv Neph & Del Gp newlv Neph & Del Gp newlv Nepl \\
\hline 1 & Case 8 - Blend 1 & 51 & TL newlv Neph & TL newlv Neph & TL newlv Neph & Del Gp newlv Neph & Del Gp newlv Nepl \\
\hline
\end{tabular}


Exhibit A1. Results for MAR Assessments of the Nominal Stage (part 4)

Constraint not met: neph (nepheline), TL (liquidus temperature), newlv (low viscosity - new model), newhv (high viscosity - new model), and Del GP - durability

\begin{tabular}{|c|c|c|c|c|c|c|c|}
\hline & & $\mathrm{Na} 2 \mathrm{O}$ & 0.11 & 0.12 & 0.13 & 0.14 & 0.15 \\
\hline Set & Type & Sludge Loading (\%) & 417 & 320 & 431 & Y1-1 & $\mathrm{X} 1-1$ \\
\hline 1 & Case 8 - Blend 1 & 52 & TL newlv Neph & TL newlv Neph & TL newlv Neph & Del Gp TL newlv Neph & Del Gp newlv Neph \\
\hline 1 & Case 8 - Blend 1 & 53 & TL newlv Neph & TL newlv Neph & TL newlv Neph & Del Gp TL newlv Neph & Del Gp TL newlv Neph \\
\hline 1 & Case 8 - Blend 1 & 54 & TL newlv Neph & TL newlv Neph & TL newlv Neph & Del Gp TL newlv Neph & Del Gp TL newlv Neph \\
\hline 1 & Case 8 - Blend 1 & 55 & TL newlv Neph & TL newlv Neph & TL newlv Neph & Del Gp TL newlv Neph & Del Gp TL newlv Neph \\
\hline 1 & Case 8 - Blend 1 & 56 & TL newlv Neph & TL newlv Neph & TL newlv Neph & Del Gp TL newlv Neph & Del Gp TL newlv Neph \\
\hline 1 & Case 8 - Blend 1 & 57 & TL newlv Neph & TL newlv Neph & TL newlv Neph & TL newlv Neph & Del Gp TL newlv Neph \\
\hline 1 & Case 8 - Blend 1 & 58 & TL newlv Neph & TL newlv Neph & TL newlv Neph & TL newlv Neph & Del Gp TL newlv Neph \\
\hline 1 & Case 8 - Blend 1 & 59 & TL newlv Neph & TL newlv Neph & TL newlv Neph & TL newlv Neph & Del Gp TL newlv Neph \\
\hline 1 & Case 8 - Blend 1 & 60 & TL newlv Neph & TL newlv Neph & TL newlv Neph & TL newlv Neph & Del Gp TL newlv Neph \\
\hline 1 & Case 8 and $8 \mathrm{a}-$ - Batch 1 & 25 & & & & Del Gp & Del Gp \\
\hline 1 & Case 8 and $8 a-$ - Batch 1 & 26 & & & & Del Gp & Del Gp \\
\hline 1 & Case 8 and $8 \mathrm{a}-$ Batch 1 & 27 & & & & Del Gp & Del Gp \\
\hline 1 & Case 8 and $8 \mathrm{a}-$ - Batch 1 & 28 & & & & Del Gp & Del Gp \\
\hline 1 & Case 8 and $8 \mathrm{a}-$ - Batch 1 & 29 & & & & Del Gp & Del Gp \\
\hline 1 & Case 8 and $8 a-$ Batch 1 & 30 & & & & Del Gp & Del Gp \\
\hline 1 & Case 8 and $8 a-$ - Batch 1 & 31 & & & & Del Gp & Del Gp \\
\hline 1 & Case 8 and $8 \mathrm{a}-$ Batch 1 & 32 & & & & Del Gp & Del Gp \\
\hline 1 & Case 8 and $8 a-$ Batch 1 & 33 & & & & & Del Gp \\
\hline 1 & Case 8 and $8 a-$ Batch 1 & 34 & & & & & Del Gp \\
\hline 1 & Case 8 and $8 \mathrm{a}-$ Batch 1 & 35 & & & & & Del Gp newlv \\
\hline 1 & Case 8 and $8 \mathrm{a}-$ - Batch 1 & 36 & & & & & Del Gp newlv Neph \\
\hline 1 & Case 8 and $8 a-$ - Batch 1 & 37 & & & & Neph & Del Gp newlv Neph \\
\hline 1 & Case 8 and $8 \mathrm{a}-$ - Batch 1 & 38 & & & & Neph & Del Gp newlv Neph \\
\hline 1 & Case 8 and $8 a-$ Batch 1 & 39 & & & Neph & Neph & Del Gp newlv Neph \\
\hline 1 & Case 8 and $8 \mathrm{a}-$ - Batch 1 & 40 & & Neph & Neph & newlv Neph & Del Gp newlv Neph \\
\hline 1 & Case 8 and $8 a-$ Batch 1 & 41 & Neph & Neph & Neph & newlv Neph & Del Gp newlv Neph \\
\hline 1 & Case 8 and $8 \mathrm{a}-$ Batch 1 & 42 & Neph & Neph & Neph & newlv Neph & Del Gp newlv Neph \\
\hline 1 & Case 8 and $8 \mathrm{a}-$ Batch 1 & 43 & Neph & Neph & Neph & newlv Neph & Del Gp newlv Neph \\
\hline 1 & Case 8 and $8 a-$ - Batch 1 & 44 & Neph & Neph & newlv Neph & newlv Neph & Del Gp newlv Neph \\
\hline 1 & Case 8 and $8 a-$ Batch 1 & 45 & Neph & Neph & newlv Neph & newlv Neph & newlv Neph \\
\hline 1 & Case 8 and $8 \mathrm{a}-$ - Batch 1 & 46 & Neph & Neph & newlv Neph & newlv Neph & newlv Neph \\
\hline 1 & Case 8 and 8 a - Batch 1 & 47 & Neph & Neph & newlv Neph & newlv Neph & newlv Neph \\
\hline 1 & Case 8 and $8 a-$ Batch 1 & 48 & Neph & newlv Neph & newlv Neph & newlv Neph & newlv Neph \\
\hline 1 & Case 8 and $8 \mathrm{a}-$ Batch 1 & 49 & TL Neph & newlv Neph & newlv Neph & newlv Neph & newlv Neph \\
\hline 1 & Case 8 and $8 a-$ - Batch 1 & 50 & TL Neph & TL newlv Neph & newlv Neph & newlv Neph & newlv Neph \\
\hline 1 & Case 8 and $8 a-$ - Batch 1 & 51 & TL Neph & TL newlv Neph & TL newlv Neph & TL newlv Neph & newlv Neph \\
\hline 1 & Case 8 and $8 a-$ Batch 1 & 52 & TL newlv Neph & TL newlv Neph & TL newlv Neph & TL newlv Neph & TL newlv Neph \\
\hline 1 & Case 8 and $8 \mathrm{a}-$ - Batch 1 & 53 & TL newlv Neph & TL newlv Neph & TL newlv Neph & TL newlv Neph & TL newlv Neph \\
\hline 1 & Case 8 and $8 \mathrm{a}-$ - Batch 1 & 54 & TL newlv Neph & TL newlv Neph & TL newlv Neph & TL newlv Neph & TL newlv Neph \\
\hline 1 & Case 8 and $8 \mathrm{a}-$ Batch 1 & 55 & TL newlv Neph & TL newlv Neph & TL newlv Neph & TL newlv Neph & TL newlv Neph \\
\hline 1 & Case 8 and $8 a-$ Batch 1 & 56 & TL newlv Neph & TL newlv Neph & TL newlv Neph & TL newlv Neph & TL newlv Neph \\
\hline 1 & Case 8 and $8 a-$ - Batch 1 & 57 & TL newlv Neph & TL newlv Neph & TL newlv Neph & TL newlv Neph & TL newlv Neph \\
\hline 1 & Case 8 and $8 a-$ Batch 1 & 58 & TL newlv Neph & TL newlv Neph & TL newlv Neph & TL newlv Neph & TL newlv Neph \\
\hline 1 & Case 8 and $8 a-$ Batch 1 & 59 & TL newlv Neph & TL newlv Neph & TL newlv Neph & TL newlv Neph & TL newlv Neph \\
\hline 1 & Case 8 and $8 a-$ Batch 1 & 60 & TL newlv Neph & TL newlv Neph & TL newlv Neph & TL newlv Neph & TL newlv Neph \\
\hline 1 & Case 8A - Blend 1 & 25 & & & & Del Gp & Del Gp \\
\hline 1 & Case $8 \mathrm{~A}$ - Blend 1 & 26 & & & & Del Gp & Del Gp \\
\hline 1 & Case $8 \mathrm{~A}$ - Blend 1 & 27 & & & & Del Gp & Del Gp \\
\hline 1 & Case 8A - Blend 1 & 28 & & & & Del Gp & Del Gp \\
\hline 1 & Case $8 \mathrm{~A}$ - Blend 1 & 29 & & & & Del Gp & Del Gp \\
\hline 1 & Case $8 \mathrm{~A}$ - Blend 1 & 30 & & & & Del Gp & Del Gp newlv \\
\hline
\end{tabular}


Exhibit A1. Results for MAR Assessments of the Nominal Stage (part 4)

Constraint not met: neph (nepheline), TL (liquidus temperature), newlv (low viscosity - new model), newhv (high viscosity - new model), and Del GP - durability

\begin{tabular}{|c|c|c|c|c|c|c|c|}
\hline & & $\mathrm{Na} 2 \mathrm{O}$ & 0.11 & 0.12 & 0.13 & 0.14 & 0.15 \\
\hline Set & $\begin{array}{c}\text { Type } \\
\end{array}$ & Sludge Loading (\%) & 417 & 320 & 431 & Y1-1 & $\mathrm{X} 1-1$ \\
\hline 1 & Case 8A - Blend 1 & 31 & & & & Del Gp & Del Gp newlv \\
\hline 1 & Case 8A - Blend 1 & 32 & & & & Del Gp & Del Gp newlv \\
\hline 1 & Case 8A - Blend 1 & 33 & & & & Del Gp & Del Gp newlv \\
\hline 1 & Case 8A - Blend 1 & 34 & & & & Del Gp & Del Gp newlv \\
\hline 1 & Case 8A - Blend 1 & 35 & & & & Del Gp newlv & Del Gp newlv \\
\hline 1 & Case 8A - Blend 1 & 36 & & & & Del Gp newlv & Del Gp newlv \\
\hline 1 & Case 8A - Blend 1 & 37 & & & & Del Gp newlv & Del Gp newlv Neph \\
\hline 1 & Case 8A - Blend 1 & 38 & & & & Del Gp newlv & Del Gp newlv Neph \\
\hline 1 & Case 8A - Blend 1 & 39 & & & newlv & Del Gp newlv Neph & Del Gp newlv Neph \\
\hline 1 & Case 8A - Blend 1 & 40 & & & newlv Neph & Del Gp newlv Neph & Del Gp newlv Neph \\
\hline 1 & Case 8A - Blend 1 & 41 & & Neph & newlv Neph & Del Gp newlv Neph & Del Gp newlv Neph \\
\hline 1 & Case 8A-Blend 1 & 42 & Neph & Neph & newlv Neph & Del Gp newlv Neph & Del Gp newlv Neph \\
\hline 1 & Case 8A - Blend 1 & 43 & Neph & newlv Neph & newlv Neph & Del Gp newlv Neph & Del Gp newlv Neph \\
\hline 1 & Case 8A - Blend 1 & 44 & Neph & newlv Neph & newlv Neph & Del Gp newlv Neph & Del Gp newlv Neph \\
\hline 1 & Case 8A - Blend 1 & 45 & Neph & newlv Neph & newlv Neph & newlv Neph & Del Gp newlv Neph \\
\hline 1 & Case 8A - Blend 1 & 46 & newlv Neph & newlv Neph & newlv Neph & newlv Neph & Del Gp newlv Neph \\
\hline 1 & Case 8A - Blend 1 & 47 & newlv Neph & newlv Neph & newlv Neph & newlv Neph & Del Gp newlv Neph \\
\hline 1 & Case 8A - Blend 1 & 48 & newlv Neph & newlv Neph & newlv Neph & newlv Neph & Del Gp newlv Neph \\
\hline 1 & Case 8A - Blend 1 & 49 & TL newlv Neph & newlv Neph & newlv Neph & newlv Neph & Del Gp newlv Neph \\
\hline 1 & Case 8A - Blend 1 & 50 & TL newlv Neph & TL newlv Neph & newlv Neph & newlv Neph & Del Gp newlv Neph \\
\hline 1 & Case 8A - Blend 1 & 51 & TL newlv Neph & TL newlv Neph & TL newlv Neph & newlv Neph & Del Gp newlv Neph \\
\hline 1 & Case 8A - Blend 1 & 52 & TL newlv Neph & TL newlv Neph & TL newlv Neph & TL newlv Neph & Del Gp newlv Neph \\
\hline 1 & Case 8A-Blend 1 & 53 & TL newlv Neph & TL newlv Neph & TL newlv Neph & TL newlv Neph & Del Gp TL newlv Neph \\
\hline 1 & Case 8A - Blend 1 & 54 & TL newlv Neph & TL newlv Neph & TL newlv Neph & TL newlv Neph & Del Gp TL newlv Neph \\
\hline 1 & Case 8A - Blend 1 & 55 & TL newlv Neph & TL newlv Neph & TL newlv Neph & TL newlv Neph & Del Gp TL newlv Neph \\
\hline 1 & Case $8 \mathrm{~A}-$ Blend 1 & 56 & TL newlv Neph & TL newlv Neph & TL newlv Neph & TL newlv Neph & Del Gp TL newlv Neph \\
\hline 1 & Case 8A - Blend 1 & 57 & TL newlv Neph & TL newlv Neph & TL newlv Neph & TL newlv Neph & Del Gp TL newlv Neph \\
\hline 1 & Case 8A - Blend 1 & 58 & TL newlv Neph & TL newly Neph & TL newlv Neph & TL newlv Neph & TL newlv Neph \\
\hline 1 & Case 8A - Blend 1 & 59 & TL newlv Neph & TL newlv Neph & TL newlv Neph & TL newlv Neph & TL newlv Neph \\
\hline 1 & Case $8 \mathrm{~A}-$ Blend 1 & 60 & TL newlv Neph & TL newlv Neph & TL newlv Neph & TL newlv Neph & TL newlv Neph \\
\hline 2 & Case 15 Batch 1 & 25 & newhv & & & & \\
\hline 2 & Case 15 Batch 1 & 26 & newhy & & & & \\
\hline 2 & Case 15 Batch 1 & 27 & newhy & & & & \\
\hline 2 & Case 15 Batch 1 & 28 & newhy & & & & \\
\hline 2 & Case 15 Batch 1 & 29 & newhv & & & & \\
\hline 2 & Case 15 Batch 1 & 30 & newhy & & & & \\
\hline 2 & Case 15 Batch 1 & 31 & newhy & & & & \\
\hline 2 & Case 15 Batch 1 & 32 & newhy & & & & Neph \\
\hline 2 & Case 15 Batch 1 & 33 & newhy & & & & Neph \\
\hline 2 & Case 15 Batch 1 & 34 & newhy & newhy & & Neph & Neph \\
\hline 2 & Case 15 Batch 1 & 35 & newhy & newhy & Neph & Neph & Neph \\
\hline 2 & Case 15 Batch 1 & 36 & newhv & newhv Neph & Neph & Neph & Neph \\
\hline 2 & Case 15 Batch 1 & 37 & newhv Neph & newhv Neph & Neph & Neph & Neph \\
\hline 2 & Case 15 Batch 1 & 38 & newhv Neph & newhv Neph & Neph & Neph & Neph \\
\hline 2 & Case 15 Batch 1 & 39 & newhv Neph & newhv Neph & Neph & Neph & Neph \\
\hline 2 & Case 15 Batch 1 & 40 & newhv Neph & newhv Neph & Neph & Neph & Neph \\
\hline 2 & Case 15 Batch 1 & 41 & newhv Neph & newhv Neph & Neph & Neph & Neph \\
\hline 2 & Case 15 Batch 1 & 42 & newhv Neph & newhy Neph & Neph & Neph & Neph \\
\hline 2 & Case 15 Batch 1 & 43 & newhv Neph & newhv Neph & Neph & Neph & Neph \\
\hline 2 & Case 15 Batch 1 & 44 & newhv Neph & newhv Neph & Neph & Neph & Neph \\
\hline 2 & Case 15 Batch 1 & 45 & newhv Neph & newhy Neph & newhv Neph & Neph & Neph \\
\hline
\end{tabular}


Exhibit A1. Results for MAR Assessments of the Nominal Stage (part 4)

Constraint not met: neph (nepheline), TL (liquidus temperature), newlv (low viscosity - new model), newhv (high viscosity - new model), and Del GP - durability

\begin{tabular}{|c|c|c|c|c|c|c|c|}
\hline & & $\mathrm{Na} 2 \mathrm{O}$ & 0.11 & 0.12 & 0.13 & 0.14 & 0.15 \\
\hline Set & Type & Sludge Loading (\%) & 417 & 320 & 431 & $\mathrm{Y} 1-1$ & $\mathrm{X} 1-1$ \\
\hline 2 & Case 15 Batch 1 & 46 & newhv Neph & newhv Neph & newhv Neph & Neph & Neph \\
\hline 2 & Case 15 Batch 1 & 47 & newhv Neph & newhv Neph & newhv Neph & Neph & Neph \\
\hline 2 & $\begin{array}{l}\text { Case } 15 \text { Batch } 1 \\
\end{array}$ & 48 & newhv Neph & newhv Neph & newhv Neph & Neph & Neph \\
\hline 2 & Case 15 Batch 1 & 49 & newhv Neph & newhv Neph & newhv Neph & Neph & Neph \\
\hline 2 & Case 15 Batch 1 & 50 & newhv Neph & newhv Neph & newhy Neph & Neph & Neph \\
\hline 2 & Case 15 Batch 1 & 51 & newhv Neph & newhy Neph & newhv Neph & Neph & Neph \\
\hline 2 & Case 15 Batch 1 & 52 & newhv Neph & newhv Neph & newhv Neph & Neph & Neph \\
\hline 2 & Case 15 Batch 1 & 53 & newhv Neph & newhv Neph & newhy Neph & newhy Neph & Neph \\
\hline 2 & Case 15 Batch 1 & 54 & newhv Neph & newhv Neph & newhv Neph & newhy Neph & Neph \\
\hline 2 & Case 15 Batch 1 & 55 & TL newhy Neph & newhv Neph & newhv Neph & newhy Neph & Neph \\
\hline 2 & Case 15 Batch 1 & 56 & TL newhv Neph & TL newhv Neph & newhv Neph & newhv Neph & Neph \\
\hline 2 & Case 15 Batch 1 & 57 & TL newhy Neph & TL newhy Neph & TL newhv Neph & TL newhy Neph & Neph \\
\hline 2 & Case 15 Batch 1 & 58 & TL newhy Neph & TL newhy Neph & TL newhy Neph & TL newhy Neph & TL Neph \\
\hline 2 & Case 15 Batch 1 & 59 & TL newhy Neph & TL newhy Neph & TL newhy Neph & TL newhv Neph & TL newhv Neph \\
\hline 2 & Case 15 Batch 1 & 60 & TL newhy Neph & TL newhv Neph & TL newhy Neph & TL newhy Neph & TL newhv Neph \\
\hline 2 & Case 15 Batch 2 & 25 & & & & Del Gp & Del Gp \\
\hline 2 & Case 15 Batch 2 & 26 & & & & Del Gp & Del Gp \\
\hline 2 & Case 15 Batch 2 & 27 & & & & Del Gp & Del Gp \\
\hline 2 & Case 15 Batch 2 & 28 & & & & Del Gp & Del Gp \\
\hline 2 & Case 15 Batch 2 & 29 & & & & Del Gp & Del Gp \\
\hline 2 & Case 15 Batch 2 & 30 & & & & Del Gp & Del Gp \\
\hline 2 & Case 15 Batch 2 & 31 & & & & Del Gp & Del Gp \\
\hline 2 & Case 15 Batch 2 & 32 & & & & Del Gp & Del Gp \\
\hline 2 & Case 15 Batch 2 & 33 & & & & Del Gp & Del Gp newlv \\
\hline 2 & Case 15 Batch 2 & 34 & & & & Del Gp & Del Gp newlv \\
\hline 2 & Case 15 Batch 2 & 35 & & & & Del Gp & Del Gp newlv Neph \\
\hline 2 & Case 15 Batch 2 & 36 & & & & Del Gp & Del Gp newlv Neph \\
\hline 2 & Case 15 Batch 2 & 37 & & & & Del Gp Neph & Del Gp newlv Neph \\
\hline 2 & Case 15 Batch 2 & 38 & & & Neph & Del Gp newlv Neph & Del Gp newlv Neph \\
\hline 2 & Case 15 Batch 2 & 39 & & Neph & Neph & Del Gp newlv Neph & Del Gp newlv Neph \\
\hline 2 & Case 15 Batch 2 & 40 & Neph & Neph & Neph & Del Gp newlv Neph & Del Gp newlv Neph \\
\hline 2 & Case 15 Batch 2 & 41 & Neph & Neph & Neph & Del Gp newlv Neph & Del Gp newlv Neph \\
\hline 2 & Case 15 Batch 2 & 42 & Neph & Neph & newlv Neph & Del Gp newlv Neph & Del Gp newlv Neph \\
\hline 2 & Case 15 Batch 2 & 43 & Neph & Neph & newlv Neph & Del Gp newlv Neph & Del Gp newlv Neph \\
\hline 2 & Case 15 Batch 2 & 44 & Neph & Neph & newlv Neph & newlv Neph & Del Gp newlv Neph \\
\hline 2 & Case 15 Batch 2 & 45 & Neph & Neph & newlv Neph & newlv Neph & Del Gp newlv Neph \\
\hline 2 & Case 15 Batch 2 & 46 & Neph & newlv Neph & newlv Neph & newlv Neph & Del Gp newlv Neph \\
\hline 2 & Case 15 Batch 2 & 47 & Neph & newlv Neph & newlv Neph & newlv Neph & Del Gp newlv Neph \\
\hline 2 & Case 15 Batch 2 & 48 & Neph & newlv Neph & newlv Neph & newlv Neph & Del Gp newlv Neph \\
\hline 2 & Case 15 Batch 2 & 49 & newlv Neph & newlv Neph & newlv Neph & newlv Neph & Del Gp newlv Neph \\
\hline 2 & Case 15 Batch 2 & 50 & TL newlv Neph & newlv Neph & newlv Neph & newlv Neph & Del Gp newlv Neph \\
\hline 2 & Case 15 Batch 2 & 51 & TL newlv Neph & TL newlv Neph & newlv Neph & newlv Neph & Del Gp newlv Neph \\
\hline 2 & Case 15 Batch 2 & 52 & TL newlv Neph & TL newlv Neph & TL newlv Neph & newlv Neph & Del Gp newlv Neph \\
\hline 2 & Case 15 Batch 2 & 53 & TL newlv Neph & TL newlv Neph & TL newlv Neph & TL newlv Neph & Del Gp newlv Neph \\
\hline 2 & Case 15 Batch 2 & 54 & TL newlv Neph & TL newlv Neph & TL newlv Neph & TL newlv Neph & Del Gp TL newlv Neph \\
\hline 2 & Case 15 Batch 2 & 55 & TL newlv Neph & TL newlv Neph & TL newlv Neph & TL newlv Neph & Del Gp TL newlv Neph \\
\hline 2 & Case 15 Batch 2 & 56 & TL newlv Neph & TL newlv Neph & TL newlv Neph & TL newlv Neph & TL newlv Neph \\
\hline 2 & Case 15 Batch 2 & 57 & TL newlv Neph & TL newlv Neph & TL newlv Neph & TL newlv Neph & TL newlv Neph \\
\hline 2 & Case 15 Batch 2 & 58 & TL newlv Neph & TL newlv Neph & TL newlv Neph & TL newlv Neph & TL newlv Neph \\
\hline 2 & Case 15 Batch 2 & 59 & TL newlv Neph & TL newlv Neph & TL newlv Neph & TL newlv Neph & TL newlv Neph \\
\hline 2 & Case 15 Batch 2 & 60 & TL newlv Neph & TL newlv Neph & TL newlv Neph & TL newlv Neph & TL newlv Neph \\
\hline
\end{tabular}


Exhibit A1. Results for MAR Assessments of the Nominal Stage (part 4)

Constraint not met: neph (nepheline), TL (liquidus temperature), newlv (low viscosity - new model), newhv (high viscosity - new model), and Del GP - durability

\begin{tabular}{|c|c|c|c|c|c|c|c|}
\hline & & $\mathrm{Na} 2 \mathrm{O}$ & 0.11 & 0.12 & 0.13 & 0.14 & 0.15 \\
\hline Set & Type & Sludge Loading (\%) & 417 & 320 & 431 & $\mathrm{Y} 1-1$ & $\mathrm{X1-1}$ \\
\hline 2 & Case 15 Blend 1 & 25 & & & & & Del Gp \\
\hline 2 & Case 15 Blend 1 & 26 & & & & & Del Gp \\
\hline 2 & Case 15 Blend 1 & 27 & & & & & Del Gp \\
\hline 2 & Case 15 Blend 1 & 28 & & & & & Del Gp \\
\hline 2 & Case 15 Blend 1 & 29 & & & & & Del Gp \\
\hline 2 & Case 15 Blend 1 & 30 & & & & & Del Gp \\
\hline 2 & Case 15 Blend 1 & 31 & & & & & Del Gp \\
\hline 2 & Case 15 Blend 1 & 32 & & & & & Del Gp newlv \\
\hline 2 & Case 15 Blend 1 & 33 & & & & & Del Gp newlv \\
\hline 2 & Case 15 Blend 1 & 34 & & & & & Del Gp newlv \\
\hline 2 & Case 15 Blend 1 & 35 & & & & & newlv \\
\hline 2 & Case 15 Blend 1 & 36 & & & & & newlv \\
\hline 2 & Case 15 Blend 1 & 37 & & & & newlv & newlv \\
\hline 2 & Case 15 Blend 1 & 38 & & & & newlv & newlv \\
\hline 2 & Case 15 Blend 1 & 39 & & & & newlv & newlv Neph \\
\hline 2 & Case 15 Blend 1 & 40 & & & & newlv & newlv Neph \\
\hline 2 & Case 15 Blend 1 & 41 & & & & newlv Neph & newlv Neph \\
\hline 2 & Case 15 Blend 1 & 42 & & & newlv Neph & newlv Neph & newlv Neph \\
\hline 2 & Case 15 Blend 1 & 43 & & Neph & newlv Neph & newlv Neph & newlv Neph \\
\hline 2 & Case 15 Blend 1 & 44 & Neph & Neph & newlv Neph & newlv Neph & newlv Neph \\
\hline 2 & Case 15 Blend 1 & 45 & Neph & newlv Neph & newlv Neph & newlv Neph & newlv Neph \\
\hline 2 & Case 15 Blend 1 & 46 & Neph & newlv Neph & newlv Neph & newlv Neph & newlv Neph \\
\hline 2 & Case 15 Blend 1 & 47 & Neph & newlv Neph & newly Neph & newlv Neph & newlv Neph \\
\hline 2 & Case 15 Blend 1 & 48 & Neph & newlv Neph & newlv Neph & newlv Neph & newlv Neph \\
\hline 2 & Case 15 Blend 1 & 49 & TL newlv Neph & newlv Neph & newlv Neph & newlv Neph & newlv Neph \\
\hline 2 & Case 15 Blend 1 & 50 & TL newlv Neph & TL newlv Neph & newlv Neph & newlv Neph & newlv Neph \\
\hline 2 & Case 15 Blend 1 & 51 & TL newlv Neph & TL newlv Neph & TL newlv Neph & newlv Neph & newlv Neph \\
\hline 2 & Case 15 Blend 1 & 52 & TL newlv Neph & TL newly Neph & TL newlv Neph & TL newlv Neph & newlv Neph \\
\hline 2 & Case 15 Blend 1 & 53 & TL newlv Neph & TL newlv Neph & TL newlv Neph & TL newlv Neph & TL newlv Neph \\
\hline 2 & Case 15 Blend 1 & 54 & TL newlv Neph & TL newlv Neph & TL newlv Neph & TL newlv Neph & TL newlv Neph \\
\hline 2 & Case 15 Blend 1 & 55 & TL newly Neph & TL newlv Neph & TL newlv Neph & TL newlv Neph & TL newlv Neph \\
\hline 2 & Case 15 Blend 1 & 56 & TL newlv Neph & TL newlv Neph & TL newlv Neph & TL newlv Neph & TL newlv Neph \\
\hline 2 & Case 15 Blend 1 & 57 & TL newlv Neph & TL newly Neph & TL newlv Neph & TL newlv Neph & TL newlv Neph \\
\hline 2 & Case 15 Blend 1 & 58 & TL newlv Neph & TL newlv Neph & TL newlv Neph & TL newlv Neph & TL newlv Neph \\
\hline 2 & Case 15 Blend 1 & 59 & TL newlv Neph & TL newlv Neph & TL newlv Neph & TL newlv Neph & TL newlv Neph \\
\hline 2 & Case 15 Blend 1 & 60 & TL newlv Neph & TL newlv Neph & TL newlv Neph & TL newlv Neph & TL newlv Neph \\
\hline 2 & Case 15 Blend 2 & 25 & & & & Del Gp & Del Gp \\
\hline 2 & Case 15 Blend 2 & 26 & & & & Del Gp & Del Gp \\
\hline 2 & Case 15 Blend 2 & 27 & & & & Del Gp & Del Gp \\
\hline 2 & Case 15 Blend 2 & 28 & & & & Del Gp & Del Gp \\
\hline 2 & Case 15 Blend 2 & 29 & & & & Del Gp & Del Gp \\
\hline 2 & Case 15 Blend 2 & 30 & & & & Del Gp & Del Gp \\
\hline 2 & Case 15 Blend 2 & 31 & & & & Del Gp & Del Gp \\
\hline 2 & Case 15 Blend 2 & 32 & & & & Del Gp & Del Gp newlv \\
\hline 2 & Case 15 Blend 2 & 33 & & & & Del Gp & Del Gp newlv \\
\hline 2 & Case 15 Blend 2 & 34 & & & & Del Gp & Del Gp newlv \\
\hline 2 & Case 15 Blend 2 & 35 & & & & Del Gp & Del Gp newlv \\
\hline 2 & Case 15 Blend 2 & 36 & & & & Del Gp & Del Gp newlv Nepl \\
\hline 2 & Case 15 Blend 2 & 37 & & & & Del Gp newlv Neph & Del Gp newlv Nepl \\
\hline 2 & Case 15 Blend 2 & 38 & & & & Del Gp newlv Neph & Del Gp newlv Nepl \\
\hline 2 & Case 15 Blend 2 & 39 & & & Neph & Del Gp newlv Neph & Del Gp newlv Nepl \\
\hline
\end{tabular}


Exhibit A1. Results for MAR Assessments of the Nominal Stage (part 4)

Constraint not met: neph (nepheline), TL (liquidus temperature), newlv (low viscosity - new model), newhv (high viscosity - new model), and Del GP - durability

\begin{tabular}{|c|c|c|c|c|c|c|c|}
\hline & & $\mathrm{Na} 2 \mathrm{O}$ & 0.11 & 0.12 & 0.13 & 0.14 & 0.15 \\
\hline Set & Type & Sludge Loading (\%) & 417 & 320 & 431 & Y1-1 & X1-1 \\
\hline 2 & Case 15 Blend 2 & 40 & & Neph & Neph & Del Gp newlv Neph & Del Gp newlv Nepl \\
\hline 2 & Case 15 Blend 2 & 41 & Neph & Neph & newlv Neph & Del Gp newlv Neph & Del Gp newlv Nepl \\
\hline 2 & Case 15 Blend 2 & 42 & Neph & Neph & newlv Neph & newlv Neph & Del Gp newlv Nepl \\
\hline 2 & Case 15 Blend 2 & 43 & Neph & Neph & newlv Neph & newlv Neph & Del Gp newlv Nepl \\
\hline 2 & Case 15 Blend 2 & 44 & Neph & Neph & newlv Neph & newlv Neph & Del Gp newlv Nepl \\
\hline 2 & Case 15 Blend 2 & 45 & Neph & newlv Neph & newlv Neph & newlv Neph & Del Gp newlv Nepl \\
\hline 2 & Case 15 Blend 2 & 46 & Neph & newlv Neph & newlv Neph & newlv Neph & Del Gp newlv Nepl \\
\hline 2 & Case 15 Blend 2 & 47 & Neph & newlv Neph & newlv Neph & newlv Neph & Del Gp newlv Nepl \\
\hline 2 & Case 15 Blend 2 & 48 & newlv Neph & newlv Neph & newlv Neph & newlv Neph & Del Gp newlv Nepl \\
\hline 2 & Case 15 Blend 2 & 49 & newlv Neph & newlv Neph & newlv Neph & newlv Neph & Del Gp newlv Nepl \\
\hline 2 & Case 15 Blend 2 & 50 & newlv Neph & newlv Neph & newlv Neph & newlv Neph & Del Gp newlv Nepl \\
\hline 2 & Case 15 Blend 2 & 51 & TL newlv Neph & newlv Neph & newlv Neph & newlv Neph & Del Gp newlv Nepl \\
\hline 2 & Case 15 Blend 2 & 52 & TL newlv Neph & TL newlv Neph & newlv Neph & newlv Neph & Del Gp newlv Nepl \\
\hline 2 & Case 15 Blend 2 & 53 & TL newlv Neph & TL newlv Neph & TL newlv Neph & TL newlv Neph & Del Gp newlv Nepl \\
\hline 2 & Case 15 Blend 2 & 54 & TL newlv Neph & TL newlv Neph & TL newlv Neph & TL newlv Neph & TL newlv Neph \\
\hline 2 & Case 15 Blend 2 & 55 & TL newlv Neph & TL newlv Neph & TL newlv Neph & TL newlv Neph & TL newlv Neph \\
\hline 2 & Case 15 Blend 2 & 56 & TL newlv Neph & TL newlv Neph & TL newlv Neph & TL newlv Neph & TL newlv Neph \\
\hline 2 & Case 15 Blend 2 & 57 & TL newlv Neph & TL newlv Neph & TL newlv Neph & TL newlv Neph & TL newlv Neph \\
\hline 2 & Case 15 Blend 2 & 58 & TL newlv Neph & TL newly Neph & TL newlv Neph & TL newlv Neph & TL newlv Neph \\
\hline 2 & Case 15 Blend 2 & 59 & TL newlv Neph & TL newlv Neph & TL newlv Neph & TL newlv Neph & TL newlv Neph \\
\hline 2 & Case 15 Blend 2 & 60 & TL newlv Neph & TL newlv Neph & TL newlv Neph & TL newlv Neph & TL newlv Neph \\
\hline 3 & Case $7 \mathrm{~b}$ Batch 1 & 25 & & & Del Gp & Del Gp & Del Gp \\
\hline 3 & Case 7b Batch 1 & 26 & & & Del Gp & Del Gp & Del Gp \\
\hline 3 & Case 7b Batch 1 & 27 & & & Del Gp & Del Gp & Del Gp \\
\hline 3 & Case 7b Batch 1 & 28 & & & Del Gp & Del Gp & Del Gp \\
\hline 3 & Case 7b Batch 1 & 29 & & & Del Gp & Del Gp & Del Gp \\
\hline 3 & Case 7b Batch 1 & 30 & & & Del Gp & Del Gp & Del Gp \\
\hline 3 & Case 7b Batch 1 & 31 & & & & Del Gp & Del Gp \\
\hline 3 & Case $7 \mathrm{~b}$ Batch 1 & 32 & & & & Del Gp & Del Gp \\
\hline 3 & Case 7b Batch 1 & 33 & & & & Del Gp & Del Gp newlv \\
\hline 3 & Case 7b Batch 1 & 34 & & & & Del Gp & Del Gp newlv Nepl \\
\hline 3 & Case $7 \mathrm{~b}$ Batch 1 & 35 & & & & Del Gp Neph & Del Gp newlv Nepl \\
\hline 3 & Case 7b Batch 1 & 36 & & & Neph & Del Gp Neph & Del Gp newlv Nepl \\
\hline 3 & Case 7b Batch 1 & 37 & & Neph & Neph & Del Gp Neph & Del Gp newlv Nepl \\
\hline 3 & Case 7b Batch 1 & 38 & & Neph & Neph & Del Gp newlv Neph & Del Gp newlv Nepl \\
\hline 3 & Case $7 \mathrm{~b}$ Batch 1 & 39 & Neph & Neph & Neph & Del Gp newlv Neph & Del Gp newlv Nepl \\
\hline 3 & Case 7b Batch 1 & 40 & Neph & Neph & Neph & Del Gp newlv Neph & Del Gp newlv Nepl \\
\hline 3 & Case 7b Batch 1 & 41 & Neph & Neph & Neph & Del Gp newlv Neph & Del Gp newlv Nepl \\
\hline 3 & Case 7b Batch 1 & 42 & Neph & Neph & Neph & $\begin{array}{l}\text { Del Gp newlv Neph } \\
\end{array}$ & Del Gp newlv Nepl \\
\hline 3 & Case $7 \mathrm{~b}$ Batch 1 & 43 & Neph & Neph & newlv Neph & Del Gp newlv Neph & Del Gp newlv Nepl \\
\hline 3 & Case 7b Batch 1 & 44 & Neph & Neph & newlv Neph & Del Gp newlv Neph & Del Gp newlv Nepl \\
\hline 3 & Case 7b Batch 1 & 45 & Neph & Neph & newlv Neph & Del Gp newlv Neph & Del Gp newlv Nepl \\
\hline 3 & Case 7b Batch 1 & 46 & Neph & newlv Neph & newlv Neph & Del Gp newlv Neph & Del Gp newlv Nepl \\
\hline 3 & Case 7b Batch 1 & 47 & Neph & newlv Neph & newlv Neph & Del Gp newlv Neph & Del Gp newlv Nepl \\
\hline 3 & Case 7b Batch 1 & 48 & Neph & newlv Neph & newlv Neph & Del Gp newlv Neph & Del Gp newlv Nepl \\
\hline 3 & Case 7b Batch 1 & 49 & Neph & newlv Neph & newlv Neph & Del Gp newlv Neph & Del Gp newlv Nepl \\
\hline 3 & Case $7 \mathrm{~b}$ Batch 1 & 50 & newlv Neph & newlv Neph & newlv Neph & Del Gp newlv Neph & Del Gp newlv Nepl \\
\hline 3 & Case 7b Batch 1 & 51 & newlv Neph & newlv Neph & newlv Neph & Del Gp newlv Neph & Del Gp newlv Nepl \\
\hline 3 & Case 7b Batch 1 & 52 & newlv Neph & newlv Neph & newlv Neph & Del Gp newlv Neph & Del Gp newlv Nepl \\
\hline 3 & Case 7b Batch 1 & 53 & newlv Neph & newlv Neph & newlv Neph & Del Gp newlv Neph & Del Gp newlv Nepl \\
\hline 3 & Case 7b Batch 1 & 54 & newlv Neph & newlv Neph & newlv Neph & Del Gp newlv Neph & Del Gp newlv Nepl \\
\hline
\end{tabular}


Exhibit A1. Results for MAR Assessments of the Nominal Stage (part 4)

Constraint not met: neph (nepheline), TL (liquidus temperature), newlv (low viscosity - new model), newhv (high viscosity - new model), and Del GP - durability

\begin{tabular}{|c|c|c|c|c|c|c|c|}
\hline & & $\mathrm{Na} 2 \mathrm{O}$ & 0.11 & 0.12 & 0.13 & 0.14 & 0.15 \\
\hline Set & Type & Sludge Loading (\%) & 417 & 320 & 431 & $\mathrm{Y} 1-1$ & X1-1 \\
\hline 3 & Case 7b Batch 1 & 55 & newlv Neph & newlv Neph & newlv Neph & newlv Neph & Del Gp newlv Nepl \\
\hline 3 & Case 7b Batch 1 & 56 & newlv Neph & newlv Neph & newlv Neph & newlv Neph & Del Gp newlv Nepl \\
\hline 3 & Case 7b Batch 1 & 57 & newlv Neph & newlv Neph & newlv Neph & newlv Neph & Del Gp newlv Nepl \\
\hline 3 & Case $7 \mathrm{~b}$ Batch 1 & 58 & TL newlv Neph & newlv Neph & newlv Neph & newlv Neph & Del Gp newlv Nepl \\
\hline 3 & Case 7b Batch 1 & 59 & TL newlv Neph & TL newly Neph & newlv Neph & newlv Neph & Del Gp newlv Nepl \\
\hline 3 & Case 7b Batch 1 & 60 & TL newlv Neph & TL newlv Neph & TL newlv Neph & newlv Neph & Del Gp newlv Nepl \\
\hline 3 & Case $7 \mathrm{~b}$ Batch 2 & 25 & & & & & Del Gp \\
\hline 3 & Case 7b Batch 2 & 26 & & & & & Del Gp \\
\hline 3 & Case 7b Batch 2 & 27 & & & & & Del Gp \\
\hline 3 & Case 7b Batch 2 & 28 & & & & & Del Gp \\
\hline 3 & Case 7b Batch 2 & 29 & & & & & \\
\hline 3 & Case 7b Batch 2 & 30 & & & & & \\
\hline 3 & Case 7b Batch 2 & 31 & & & & & \\
\hline 3 & Case 7b Batch 2 & 32 & & & & & \\
\hline 3 & Case $7 \mathrm{~b}$ Batch 2 & 33 & & & & & \\
\hline 3 & Case 7b Batch 2 & 34 & & & & & \\
\hline 3 & Case 7b Batch 2 & 35 & & & & & Neph \\
\hline 3 & Case $7 \mathrm{~b}$ Batch 2 & 36 & & & & Neph & Neph \\
\hline 3 & Case 7b Batch 2 & 37 & & & Neph & Neph & Neph \\
\hline 3 & Case 7b Batch 2 & 38 & & & Neph & Neph & Neph \\
\hline 3 & Case 7b Batch 2 & 39 & & Neph & Neph & Neph & Neph \\
\hline 3 & Case 7b Batch 2 & 40 & Neph & Neph & Neph & Neph & Neph \\
\hline 3 & Case 7b Batch 2 & 41 & Neph & Neph & Neph & Neph & Neph \\
\hline 3 & Case 7b Batch 2 & 42 & Neph & Neph & Neph & Neph & Neph \\
\hline 3 & Case 7b Batch 2 & 43 & Neph & Neph & Neph & Neph & Neph \\
\hline 3 & Case 7b Batch 2 & 44 & Neph & Neph & Neph & Neph & Neph \\
\hline 3 & Case 7b Batch 2 & 45 & Neph & Neph & Neph & Neph & Neph \\
\hline 3 & Case 7b Batch 2 & 46 & Neph & Neph & Neph & Neph & newlv Neph \\
\hline 3 & Case $7 \mathrm{~b}$ Batch 2 & 47 & Neph & Neph & Neph & Neph & newlv Neph \\
\hline 3 & Case 7b Batch 2 & 48 & Neph & Neph & Neph & Neph & newlv Neph \\
\hline 3 & Case 7b Batch 2 & 49 & Neph & Neph & Neph & Neph & newlv Neph \\
\hline 3 & Case 7b Batch 2 & 50 & TL Neph & Neph & Neph & Neph & newlv Neph \\
\hline 3 & Case 7b Batch 2 & 51 & TL Neph & TL Neph & TL Neph & newlv Neph & newlv Neph \\
\hline 3 & Case 7b Batch 2 & 52 & TL Neph & TL Neph & TL Neph & TL newlv Neph & newlv Neph \\
\hline 3 & Case 7b Batch 2 & 53 & TL Neph & TL Neph & TL Neph & TL newlv Neph & TL newlv Neph \\
\hline 3 & Case $7 \mathrm{~b}$ Batch 2 & 54 & TL Neph & TL Neph & TL Neph & TL newlv Neph & TL newlv Neph \\
\hline 3 & Case 7b Batch 2 & 55 & TL Neph & TL Neph & TL Neph & TL newlv Neph & TL newlv Neph \\
\hline 3 & Case 7b Batch 2 & 56 & TL Neph & TL Neph & TL newlv Neph & TL newlv Neph & TL newlv Neph \\
\hline 3 & Case 7b Batch 2 & 57 & TL Neph & TL Neph & TL newlv Neph & TL newlv Neph & TL newlv Neph \\
\hline 3 & Case $7 \mathrm{~b}$ Batch 2 & 58 & TL Neph & TL Neph & TL newlv Neph & TL newlv Neph & TL newlv Neph \\
\hline 3 & Case 7b Batch 2 & 59 & TL Neph & TL newlv Neph & TL newlv Neph & TL newlv Neph & TL newlv Neph \\
\hline 3 & Case 7b Batch 2 & 60 & TL Neph & TL newlv Neph & TL newlv Neph & TL newlv Neph & TL newlv Neph \\
\hline 3 & Case $7 \mathrm{~b}$ Blend 1 & 25 & & & Del Gp & Del Gp & Del Gp newlv \\
\hline 3 & Case $7 b$ Blend 1 & 26 & & & Del Gp & Del Gp & Del Gp newlv \\
\hline 3 & Case 7b Blend 1 & 27 & & & Del Gp & Del Gp & Del Gp newlv \\
\hline 3 & Case $7 \mathrm{~b}$ Blend 1 & 28 & & & Del Gp & Del Gp & Del Gp newlv \\
\hline 3 & Case $7 \mathrm{~b}$ Blend 1 & 29 & & & Del Gp & Del Gp newlv & Del Gp newlv \\
\hline 3 & Case 7b Blend 1 & 30 & & & Del Gp & Del Gp newlv & Del Gp newlv \\
\hline 3 & Case 7b Blend 1 & 31 & & & Del Gp & Del Gp newlv & Del Gp newlv \\
\hline 3 & Case $7 \mathrm{~b}$ Blend 1 & 32 & & & Del Gp & Del Gp newlv & Del Gp newlv \\
\hline 3 & Case $7 \mathrm{~b}$ Blend 1 & 33 & & & Del Gp newlv & Del Gp newlv & Del Gp newlv \\
\hline
\end{tabular}


Exhibit A1. Results for MAR Assessments of the Nominal Stage (part 4)

Constraint not met: neph (nepheline), TL (liquidus temperature), newlv (low viscosity - new model), newhv (high viscosity - new model), and Del GP - durability

\begin{tabular}{|c|c|c|c|c|c|c|c|}
\hline & & $\mathrm{Na} 2 \mathrm{O}$ & 0.11 & 0.12 & 0.13 & 0.14 & 0.15 \\
\hline Set & Type & Sludge Loading (\%) & 417 & 320 & 431 & $\mathrm{Y} 1-1$ & $\mathrm{X} 1-1$ \\
\hline 3 & Case $7 \mathrm{~b}$ Blend 1 & 34 & & & Del Gp newlv & Del Gp newlv & Del Gp newlv \\
\hline 3 & Case 7b Blend 1 & 35 & & & Del Gp newlv & Del Gp newlv & Del Gp newlv \\
\hline 3 & Case $7 \mathrm{~b}$ Blend 1 & 36 & & newlv & Del Gp newlv & Del Gp newlv & Del Gp newlv \\
\hline 3 & Case $7 \mathrm{~b}$ Blend 1 & 37 & & newlv & Del Gp newlv & Del Gp newlv & Del Gp newlv \\
\hline 3 & Case 7b Blend 1 & 38 & & newlv & Del Gp newlv & Del Gp newlv & Del Gp newlv \\
\hline 3 & Case 7b Blend 1 & 39 & newlv & newlv & Del Gp newlv & Del Gp newlv & Del Gp newlv \\
\hline 3 & Case $7 \mathrm{~b}$ Blend 1 & 40 & newlv & newlv & Del Gp newlv & Del Gp newlv & Del Gp newlv Neph \\
\hline 3 & Case $7 \mathrm{~b}$ Blend 1 & 41 & newlv & newlv & Del Gp newlv & Del Gp newlv Neph & Del Gp newlv Neph \\
\hline 3 & Case 7b Blend 1 & 42 & newlv & newlv & Del Gp newlv Neph & Del Gp newlv Neph & Del Gp newlv Neph \\
\hline 3 & Case 7b Blend 1 & 43 & newlv & newlv & Del Gp newlv Neph & Del Gp newlv Neph & Del Gp newlv Neph \\
\hline 3 & Case $7 \mathrm{~b}$ Blend 1 & 44 & newlv & newlv Neph & Del Gp newlv Neph & Del Gp newlv Neph & Del Gp newlv Neph \\
\hline 3 & Case $7 \mathrm{~b}$ Blend 1 & 45 & newlv Neph & newlv Neph & Del Gp newlv Neph & Del Gp newlv Neph & Del Gp newlv Neph \\
\hline 3 & Case 7b Blend 1 & 46 & newlv Neph & newlv Neph & Del Gp newlv Neph & Del Gp newlv Neph & Del Gp newlv Neph \\
\hline 3 & Case 7b Blend 1 & 47 & newlv Neph & newlv Neph & Del Gp newlv Neph & Del Gp newlv Neph & Del Gp newlv Neph \\
\hline 3 & Case $7 \mathrm{~b}$ Blend 1 & 48 & newlv Neph & newlv Neph & Del Gp newlv Neph & Del Gp newlv Neph & Del Gp newlv Neph \\
\hline 3 & Case 7b Blend 1 & 49 & newlv Neph & newlv Neph & Del Gp newlv Neph & Del Gp newlv Neph & Del Gp newlv Neph \\
\hline 3 & Case 7b Blend 1 & 50 & newlv Neph & newlv Neph & Del Gp newlv Neph & Del Gp newlv Neph & Del Gp newlv Neph \\
\hline 3 & Case $7 \mathrm{~b}$ Blend 1 & 51 & newlv Neph & newlv Neph & Del Gp newlv Neph & Del Gp newlv Neph & Del Gp newlv Neph \\
\hline 3 & Case $7 \mathrm{~b}$ Blend 1 & 52 & TL newlv Neph & newlv Neph & Del Gp newlv Neph & Del Gp newlv Neph & Del Gp newlv Neph \\
\hline 3 & Case 7b Blend 1 & 53 & TL newlv Neph & TL newlv Neph & Del Gp newlv Neph & Del Gp newlv Neph & Del Gp newlv Neph \\
\hline 3 & Case 7b Blend 1 & 54 & TL newlv Neph & TL newlv Neph & Del Gp TL newlv Neph & Del Gp newlv Neph & Del Gp newlv Neph \\
\hline 3 & Case 7b Blend 1 & 55 & TL newlv Neph & TL newlv Neph & Del Gp TL newlv Neph & Del Gp TL newlv Neph & Del Gp newlv Neph \\
\hline 3 & Case 7b Blend 1 & 56 & TL newlv Neph & TL newlv Neph & Del Gp TL newlv Neph & Del Gp TL newlv Neph & Del Gp TL newlv Neph \\
\hline 3 & Case 7b Blend 1 & 57 & TL newlv Neph & TL newlv Neph & Del Gp TL newlv Neph & Del Gp TL newlv Neph & Del Gp TL newlv Neph \\
\hline 3 & Case 7b Blend 1 & 58 & TL newlv Neph & TL newlv Neph & Del Gp TL newlv Neph & Del Gp TL newlv Neph & Del Gp TL newlv Neph \\
\hline 3 & Case $7 \mathrm{~b}$ Blend 1 & 59 & TL newlv Neph & TL newlv Neph & Del Gp TL newlv Neph & Del Gp TL newlv Neph & Del Gp TL newlv Neph \\
\hline 3 & Case 7b Blend 1 & 60 & TL newlv Neph & TL newlv Neph & Del Gp TL newlv Neph & Del Gp TL newlv Neph & Del Gp TL newlv Neph \\
\hline 3 & Case 7b Blend 2 & 25 & & & & Del Gp & Del Gp \\
\hline 3 & Case $7 \mathrm{~b}$ Blend 2 & 26 & & & & & Del Gp \\
\hline 3 & Case $7 \mathrm{~b}$ Blend 2 & 27 & & & & & Del Gp \\
\hline 3 & Case 7b Blend 2 & 28 & & & & & Del Gp \\
\hline 3 & Case $7 \mathrm{~b}$ Blend 2 & 29 & & & & & Del Gp \\
\hline 3 & Case $7 \mathrm{~b}$ Blend 2 & 30 & & & & & Del Gp \\
\hline 3 & Case 7b Blend 2 & 31 & & & & & Del Gp \\
\hline 3 & Case $7 \mathrm{~b}$ Blend 2 & 32 & & & & & Del Gp \\
\hline 3 & Case $7 \mathrm{~b}$ Blend 2 & 33 & & & & & Del Gp \\
\hline 3 & Case $7 \mathrm{~b}$ Blend 2 & 34 & & & & & Del Gp \\
\hline 3 & Case 7b Blend 2 & 35 & & & & & Del Gp \\
\hline 3 & Case 7b Blend 2 & 36 & & & & & Del Gp Neph \\
\hline 3 & Case $7 \mathrm{~b}$ Blend 2 & 37 & & & & Neph & Neph \\
\hline 3 & Case 7b Blend 2 & 38 & & & Neph & Neph & newlv Neph \\
\hline 3 & Case 7b Blend 2 & 39 & & Neph & Neph & Neph & newlv Neph \\
\hline 3 & Case $7 \mathrm{~b}$ Blend 2 & 40 & & Neph & Neph & Neph & newlv Neph \\
\hline 3 & Case $7 \mathrm{~b}$ Blend 2 & 41 & Neph & Neph & Neph & Neph & newlv Neph \\
\hline 3 & Case 7b Blend 2 & 42 & Neph & Neph & Neph & Neph & newlv Neph \\
\hline 3 & Case $7 \mathrm{~b}$ Blend 2 & 43 & Neph & Neph & Neph & newlv Neph & newlv Neph \\
\hline 3 & Case $7 \mathrm{~b}$ Blend 2 & 44 & Neph & Neph & Neph & newlv Neph & newlv Neph \\
\hline 3 & Case 7b Blend 2 & 45 & Neph & Neph & Neph & newlv Neph & newlv Neph \\
\hline 3 & Case $7 \mathrm{~b}$ Blend 2 & 46 & Neph & Neph & Neph & newlv Neph & newlv Neph \\
\hline 3 & Case $7 \mathrm{~b}$ Blend 2 & 47 & Neph & Neph & newlv Neph & newlv Neph & newlv Neph \\
\hline 3 & Case $7 \mathrm{~b}$ Blend 2 & 48 & Neph & Neph & newlv Neph & newlv Neph & newlv Neph \\
\hline
\end{tabular}




\section{Exhibit A1. Results for MAR Assessments of the Nominal Stage (part 4)}

Constraint not met: neph (nepheline), TL (liquidus temperature), newlv (low viscosity - new model), newhv (high viscosity - new model), and Del GP - durability

\begin{tabular}{|c|c|c|c|c|c|c|c|}
\hline & & $\mathrm{Na} 2 \mathrm{O}$ & 0.11 & 0.12 & 0.13 & 0.14 & 0.15 \\
\hline Set & Type & Sludge Loading (\%) & 417 & 320 & 431 & Y1-1 & $\mathrm{X} 1-1$ \\
\hline 3 & Case $7 \mathrm{~b}$ Blend 2 & 49 & Neph & Neph & newlv Neph & newlv Neph & newlv Neph \\
\hline 3 & Case $7 \mathrm{~b}$ Blend 2 & 50 & Neph & Neph & newlv Neph & newlv Neph & newlv Neph \\
\hline 3 & Case $7 \mathrm{~b}$ Blend 2 & 51 & TL Neph & newlv Neph & newlv Neph & newlv Neph & newlv Neph \\
\hline 3 & Case $7 \mathrm{~b}$ Blend 2 & 52 & TL Neph & TL newlv Neph & TL newlv Neph & newlv Neph & newlv Neph \\
\hline 3 & Case $7 \mathrm{~b}$ Blend 2 & 53 & TL Neph & TL newly Neph & TL newlv Neph & TL newlv Neph & newlv Neph \\
\hline 3 & Case $7 \mathrm{~b}$ Blend 2 & 54 & TL newlv Neph & TL newlv Neph & TL newlv Neph & TL newlv Neph & TL newly Neph \\
\hline 3 & Case $7 \mathrm{~b}$ Blend 2 & 55 & TL newlv Neph & TL newlv Neph & TL newlv Neph & TL newlv Neph & TL newlv Neph \\
\hline 3 & Case $7 \mathrm{~b}$ Blend 2 & 56 & TL newlv Neph & TL newlv Neph & TL newlv Neph & TL newlv Neph & TL newlv Neph \\
\hline 3 & Case $7 \mathrm{~b}$ Blend 2 & 57 & TL newlv Neph & TL newlv Neph & TL newlv Neph & TL newlv Neph & TL newlv Neph \\
\hline 3 & Case $7 \mathrm{~b}$ Blend 2 & 58 & TL newlv Neph & TL newlv Neph & TL newlv Neph & TL newlv Neph & TL newlv Neph \\
\hline 3 & Case $7 \mathrm{~b}$ Blend 2 & 59 & TL newlv Neph & TL newlv Neph & TL newlv Neph & TL newlv Neph & TL newlv Neph \\
\hline 3 & Case $7 \mathrm{~b}$ Blend 2 & 60 & TL newlv Neph & TL newlv Neph & TL newlv Neph & TL newlv Neph & TL newlv Neph \\
\hline
\end{tabular}




\section{Distribution:}

J.E. Marra, SRNL

R.E. Edwards, SRNL

D. A. Crowley, 999-W

T. B. Calloway, 999-W

N. E. Bibler, SRNL

C.M. Jantzen, SRNL

J. R. Harbour, 773-42A

G. G. Wicks, SRNL

R. C. Tuckfield, 773-42A

D. K. Peeler, 999-W

T. B. Edwards, 773-42A

C. C. Herman, 773-42A

M. E. Smith, 773-42A

M. E. Stone, 999-W

D. H. Miller, 999-W

M. J. Barnes, 999-W

K.M. Fox, SRNL

M. S. Miller, 704-S

J. E. Occhipinti, 704-S

P. M. Patel, 704-27S

H. H. Elder, 766-H

J. F. Iaukea, 704-30S

J. W. Ray, 704-S

M. A. Rios-Armstrong, 766-H

W. B. Van-Pelt, 704-S

H. B. Shah, 766-H

J. M. Gillam, 766-H

B. A. Davis, 704-27S 